\title{
RF/Fiber Optical Interface for Microcellular Wireless Transceivers
}

\author{
by \\ Samy Ghoniemy,B.Sc., M.Sc.,
}

A Thesis Submitted to

The Faculty of Graduate Studies and Research

in partial fulfillment of the requirements for the degree of

\section{Doctor of Philosophy}

Ottawa-Carleton Institute for Electrical and Computer Engineering

Faculty of Engineering

Department of Systems and Computer Engineering

Carleton University

Ottawa, Ontario, Canada

November, 2003

(C)2003 Samy Ghoniemy 


\author{
National Library \\ of Canada \\ Acquisitions and \\ Bibliographic Services \\ 395 Wellington Street \\ Ottawa ON K1A ON4 \\ Canada
}

Bibliothèque nationale

du Canada

Acquisisitons et services bibliographiques

395 , rue Wellington Ottawa ON K1A ON4 Canada
Your file Votre référence ISBN: 0-612-89890-3

Ourfile Notre référence ISBN: 0-612-89890-3
The author has granted a nonexclusive licence allowing the National Library of Canada to reproduce, loan, distribute or sell copies of this thesis in microform, paper or electronic formats.

The author retains ownership of the copyright in this thesis. Neither the thesis nor substantial extracts from it may be printed or otherwise reproduced without the author's permission.
L'auteur a accordé une licence non exclusive permettant à la Bibliothèque nationale du Canada de reproduire, prêter, distribuer ou vendre des copies de cette thèse sous la forme de microfiche/film, de reproduction sur papier ou sur format électronique.

L'auteur conserve la propriété du droit d'auteur qui protège cette thèse. $\mathrm{Ni}$ la thèse ni des extraits substantiels de celle-ci ne doivent être imprimés ou aturement reproduits sans son autorisation.
In compliance with the Canadian Privacy Act some supporting forms may have been removed from this dissertation.

While these forms may be included in the document page count, their removal does not represent any loss of content from the dissertation.
Conformément à la loi canadienne sur la protection de la vie privée, quelques formulaires secondaires ont été enlevés de ce manuscrit.

Bien que ces formulaires aient inclus dans la pagination, il n'y aura aucun contenu manquant. 


\section{Abstract}

In this thesis, we are interested in the $\mathrm{RF} /$ Fiber optical interface for microcellular wireless transceivers as an essential part of the whole communications link. The key element in this research is the telecommunication semiconductor laser diode. However, the inhibiting factor for analog applications is the natural nonlinearity exhibited by semiconductor lasers, which degrades the overall system performance and capabilities.

Performance evaluation and analysis of the communications link before implementation is extremely desirable; however, the device fabrication is excessively, costly and too time consuming for this purpose. Rather, robust, fast and accurate semiconductor laser simulation capability will give a way for more complex and diverse laser design, characterization and performance evaluation. This requires comprehensive laser modeling.

In this thesis, semiconductor laser gain modification, and the effects of device heating, leakage and noise were studied and added simultaneously to conventional laser models. Suitable model implementations for device and systems simulations in both frequency and time domains are also presented. Laser's nonlinearity was analyzed in detail as a prelude to choosing the pre-distorter most appropriate linearization technique. Model verification and validation were then performed by comparing the simulation results with the measurement results.

Analytical expressions for the pre-distorter model in time and frequency domains are also derived. The performance of the pre-distorter was evaluated by a software implementation of the frequency domain models of both the pre-distorter and the proposed laser model in cascade. 
Simulation results show that the proposed pre-distorter improves the laser diode's nonlinear performance as measured by harmonic and intermodulation distortion. Comparison between the conventional gain-based pre-distorter and the proposed modified gain-based pre-distorter shows that the performance of the laser and pre-distortion circuit combination, in which the pre-distortion circuit is designed based upon the modified gain formulation, provides remarkable improvement over implementation based on previous laser models. Typically, an improvement of $10 \mathrm{~dB}$ in intermodulation distortion can be realized based on the work presented in this thesis, using the pre-distortion circuit designed based upon the modified gain formulation. 


\section{Acknowledgements}

I would like to express my gratitude and deep thanks to my supervisor Prof. Samy Mahmoud, Dean of the Faculty of Engineering and Design, for his guidance and encouragement. I'm greatly indebted to Prof. Mahmoud for his help in every aspect of my life during the years of my study at Carleton University. His great dedication, talent and generosity will always remain a source of inspiration to me.

I would also like to express my special thanks to Prof. Leonard MacEachern, Co-supervisor of this thesis, who was also my mentor during my doctoral research. His kind personality, support and help, as well as his helpful suggestions and comments, are highly appreciated. Most of this research was completed with his active involvement.

I am grateful to the Department of Systems and Computer Engineering for providing a suitable research environment, and for their kind assistance and help. Special thanks goes to Narendra Mehta, Computer Systems Manager, for his quick and professional help in solving computer and network problems. I would also like to express my deep thanks to Danny Lemay, Daren Russ, David Sword, John Knox, Darlene Hebert, Blazenka Power and the rest of the staff for their continuous help in providing a quiet research environment. Thanks are extended to Yvonne Clevers, Anne Waddell and Sheila McGowan for their support. I really enjoyed dealing with them.

I would like to express my appreciation to the Canadian Research Council (CRC), and especially Dr. Francois Gouin for his assistance and support. This research would not have been possible without the financial support of the Egyptian Ministry of Defense.

I would like to express my appreciation to Mr. Nagui Mikhail for his highly qualified assistance in preparing the measurement lab. I really enjoyed his company. My appreciation is also extended to my colleagues Dr. Mohamed Hossam and Dr. Jamal Hijres. Their great personalities, encouragement and fruitful discussions will never be forgotten. Thanks are 
extended to my colleagues from MTC for their discussions and encouragement. It is also essential to thank Dr. Al-Leithy, who I consider an older brother, for his guidance and sincere help.

I deeply thank my uncle Prof. Said Ghoniemy for his support, guidance, help and encouragement in every aspect of my life. He will remain in my heart all my life. Special thanks go to my uncles Helmy and Mahrouse who have also supported me in this endeavor.

I would also like to express my deep thanks to my mother. It would be impossible to recognize in detail the enormous amount of help from her.

I am indebted to my wife for all the sacrifices she has had to make over the years to help and support me while I attended graduate studies. I Also thank my daughters, Aml, Ayah and Basant for their understanding when I didn't have the time for them. Completion of the research and the writing of this dissertation would not have been possible without their love and support.

And Finally, thanks to my father, who passed away before I could realize this dream.

Ottawa, Ontario

Samy Ghoniemy

November 2003 


\section{Table of Contents}

Abstract iv

Acknowledgements vi

Table of Contents viii

List of Tables

List of Figures xii

1 Introduction 1

1.1 Introduction . . . . . . . . . . . . . . . . . . . 1

1.2 Thesis motivation . . . . . . . . . . . . . . . . . . . . 2

1.3 Thesis objectives . . . . . . . . . . . . . . . . . . . . 2

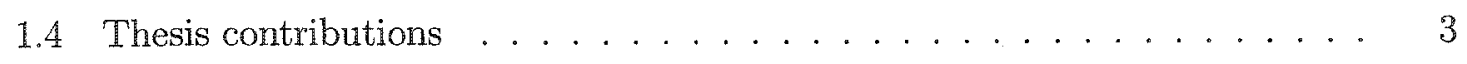

1.5 Thesis organization ..................... 4

2 Semiconductor-Diode Laser Modeling 5

2.1 Historical background . . . . . . . . . . . . . . . . 5

2.2 Basic principles of semiconductor lasers . . . . . . . . . . . 6

2.3 Semiconductor laser modeling . . . . . . . . . . . . . . . 8

2.4 Conventional semiconductor laser rate equations . . . . . . . . . . . . 10

2.5 Modified semiconductor laser rate equations . . . . . . . . . . . . . 11

2.5.1 Modified semiconductor laser gain formulation . . . . . . . . . . . 11

2.5.2 Semiconductor laser model with modified gain . . . . . . . . . 13

2.5.3 Threshold lasing condition and steady state solution . . . . . . . . 14

2.6 Laser model implementation . . . . . . . . . . . . . . . . . 15

2.6.1 Semiconductor laser diode modeling using SIMULINK . . . . . . 15

2.6 .2 Normalized rate equations . . . . . . . . . . . . . . . . 16

2.6.2.1 Normalized photon rate equation . . . . . . . . 16 
2.6.2.2 Normalized electron rate equation. . . . . . . . . 16

2.6.3 Symbolically defined laser diode models . . . . . . . . . . . . 17

2.7 Simulation results . . . . . . . . . . . . . . . . . . . 18

2.7.1 Time domain steady state simulation results . . . . . . . . . . 18

2.7.1.1 Parameters extraction ............... 18

2.7.1.2 Light-Current characteristics . . . . . . . . . . 20

2.7.1.3 Variation of carrier population ............ 22

2.7.2 Time domain dynamic simulation results . . . . . . . . . . . 22

2.7.3 Frequency domain simulation results . . . . . . . . . . . . . . 27

2.8 Model advantages and limitations . . . . . . . . . . . . . 31

3 Modified Semiconductor Laser Models 32

3.1 Semiconductor laser models incorporating thermal effects . . . . . . . . . 33

3.1.1 Rate equations temperature dependance . . . . . . . . . . 33

3.1.1.1 Transparent carrier density temperature dependance . . . 34

3.1.1.2 Laser threshold temperature dependance . . . . . . . . . 35

3.1.1.3 Gain formulation including thermal effects . . . . . . . . 36

3.1.1.4 Temperature dependance of the carrier recombination time 37

3.1.1.5 Normalized modified rate equations including thermal effects 37

3.1.2 Model implementation using symbolically defined devices . . . . . 38

3.1 .3 Simulation results . . . . . . . . . . . . . . . . 39

3.2 Enhanced thermally modified models by including leakage current effects . 45

3.2 .1 Simulation results . . . . . . . . . . . . . . . . . . . . 47

3.3 Modified laser models including noise fluctuations . . . . . . . . . . 52

3.3 .1 Noise driven enhanced laser model . . . . . . . . . . . . . . . 53

3.3.2 Noise generation technique . . . . . . . . . . . . . . 54

3.3.3 Enhanced SDD model implementation ............ 55

3.3 .4 Simulation results . . . . . . . . . . . . . . 55

3.3.4.1 Carrier, photon, and power results ........ 57

3.3.4.2 Laser intensity noise . . . . . . . . . . . . . . . . . . . . . . . . . . . . .

3.3.4.3 Laser phase/frequency noise . . . . . . . . . . . 63

3.3 .5 Summary and model advantages . . . . . . . . . . . 65

4 Experimentation and Model Verification $\quad 67$

4.1 DC measurements . . . . . . . . . . . . . . . . . . . 67

4.2 Modulation response and linearity . . . . . . . . . . . . . 69 
4.2.1 Modulation frequency response ... . . . . . . . . . . . 69

4.2 .2 Distortion measurements . . . . . . . . . . . . . 71

4.2.2.1 Harmonic response . . . . . . . . . . . . . . 71

4.2.2.2 Intermodulation distortion response .......... 73

4.3 Noise measurements . . . . . . . . . . . . . . . 76

4.3.1 RIN measurements . . . . . . . . . . . . . . . . 76

4.3 .2 Laser linewidth measurements . . . . . . . . . . . . . . . . . 79

4.4 Model verification . . . . . . . . . . . . . . . . . . 82

5 Nonlinearity and Linearization Techniques 90

5.1 Concepts of nonlinearity . . . . . . . . . . . . . . . . 90

5.1 .1 Harmonic generation . . . . . . . . . . . . . . . 9 90

5.1 .2 Nonlinearity in semiconductor lasers . . . . . . . . . . . . 91

5.2 Review of linearization techniques . . . . . . . . . . . . . 91

5.2 .1 Optoelectronic feedback . . . . . . . . . . . . . . 93

5.2 .2 Optical feed-forward . . . . . . . . . . . . . . 93

5.2 .3 Phase shift modulation . . . . . . . . . . . . . 95

5.2 .4 External light injection . . . . . . . . . . . . . . . . 96

5.2 .5 Dual-parallel modulation . . . . . . . . . . . . . . . . 96

5.2 .6 Cascaded linearized modulator . . . . . . . . . . . . 97

5.2 .7 Post-distortion . . . . . . . . . . . . . . . . 97

5.2 .8 Pre-distortion linearizer . . . . . . . . . . . . . . 98

5.2 .9 Adaptive pre-distortion . . . . . . . . . . . . . . . 99

5.3 Summary . . . . . . . . . . . . . . . . . . . . . . . 101

6 Pre-distorter Modeling, Implementation and Nonlinearity Evaluation 103

6.1 Inverse laser modeling . . . . . . . . . . . . . . . . . . 103

6.2 Frequency domain laser's pre-distorter Volterra model . . . . . . . . . 107

6.3 Time domain pre-distorter Volterra models . . . . . . . . . . . . 108

6.4 Proposed linearization technique and model . . . . . . . . . . . . . 111

6.5 Pre-distorter implementation and nonlinearity evaluation . . . . . . . 113

6.5.1 Frequency domain semiconductor laser Volterra model . . . . . . 113

6.5 .2 Distortion evaluation . . . . . . . . . . . . . . 116

6.5.2.1 Distortion evaluation without using pre-distorter . . . . 117

6.5.2.2 Distortion evaluation after pre-distorter . . . . . . . . . 119

6.5.3 Pre-distorter Implementation and Testing . . . . . . . . . . . . . 124 
Appendix A Semiconductor Laser Gain 142

A.1 Index of refraction and absorption coefficient . . . . . . . . . . . . 144

A.2 Effect of refractive index variation on the Gain . . . . . . . . . . 145

A.3 Threshold condition . . . . . . . . . . . . . . . 146

A.4 Index nonlinearity effect on the laser gain . . . . . . . . . . . . 146

A.4.1 Linear change of refractive index and gain . . . . . . . . . . 147

A.4.2 Nonlinear change of refractive index and gain . . . . . . . . . 147

A.5 Total gain function . . . . . . . . . . . . . . . . 147

Appendix B Transparent Carricr Density Temperature Dependance 148

Appendix C Temperature Dependance of the Group Velocity 151

Appendix D Leakage Current 152

Appendix E Quantum Theory of Laser and Noise Generation 154

E.1 Langevin equation and Einstein relations . . . . . . . . . . . . . 155

E.2 Autocorrelation of the atomic noise operators . . . . . . . . . . 156

E.3 Noise driven laser rate equations . . . . . . . . . . . . . . . 156

E.4 Independent carrier noise source generation . . . . . . . . . . . . 158

E.5 Auto and cross-correlation relations . . . . . . . . . . . 160

Appendix F Volterra Series 162

F.1 Introduction to Volterra Series . . . . . . . . . . . . . . . . . . 162

F.2 Concepts of Volterra series . . . . . . . . . . . . . . . 162

F.3 Volterra kernels determination . . . . . . . . . . . . . . . 164

F.3.1 Harmonic-Input (probing) method . . . . . . . . . . . . 164

F.3.2 Direct expansion method . . . . . . . . . . . . . 164

Appendix G Measurement Techniques $\quad \mathbf{1 6 6}$

G.1 DC measurement technique . . . . . . . . . . . . . . 166

G.2 Modulation response measurement technique . . . . . . . . . . . 167

G.3 Distortion measurement technique . . . . . . . . . . . . 168

G.4 Parameters extraction algorithm . . . . . . . . . . . . . 169

$\begin{array}{lr}\text { Bibliography } & \mathbf{1 7 2}\end{array}$ 


\section{List of Tables}

2.1 Device Parameters for InGaAsP/InP semiconductor laser diodes . . . . 20

2.2 Extracted Parameters . . . . . . . . . . . . . . . . . 20

3.1 Parameters for an InGaAsP/InP semiconductor laser diode and other general physical constants . . . . . . . . . . . . . . . . . . . 39

5.1 Summary of the linearization techniques and distortion reduction results. . 101

6.1 Parameters values for 1.3 $\mu m$ InGaAsP/InP semiconductor Ortel $1510 \mathrm{~B}$ and Fujitsu FLD3F7CZ laser diodes . . . . . . . . . . . . . . . 125

G.1 Extracted parameters for a $1.3 \mu \mathrm{m}$ InGaAsP/InP Fujitsu FLD3F7CZ semiconductor laser. . . . . . . . . . . . . . . . . . . . 171 


\section{List of Figures}

2.1 Two-energy-level diagrams . . . . . . . . . . . . . . . 7

2.2 SIMULINK block diagram representation of semiconductor laser rate equations. [1] . . . . . . . . . . . . . . . . . . 15

2.3 The schematic symbol and port equations for a 3-port SDD [2]. . . . . . 19

2.4 Agilent-ADS schematic diagram for the simulation setup. The diode symbol shown represents the hierarchically defined SDD shown in Figure 2.3. The output power is represented by a current in this simulation. . . . . . . . . . 19

2.5 Normalized photon density Vs normalized derive current and the corresponding optical output power $\mathrm{Vs}$ derive current. As shown, the spontaneous coupling factor $\beta_{s p}$ has a noticeable effect on the light current curve.

2.6 Steady state electron density $\mathbb{C} V_{s}$ derive current $I(t)$ and the normalized values $\mathbb{C}_{n}$ Vs the normalized drive current. . . . . . . . . . . . 22

2.7 Time evolution of the normalized carrier density. . . . . . . . . . . . 23

2.8 Time evolution of the normalized photon density. . . . . . . . . . . . 24

2.9 Dynamics of the carrier and photon population in time domain showing the turn on spikes and the transient period. . . . . . . . . . . . . . . 24

2.10 First spike from the dynamics of the carrier and photon population shown in Figure 2.9. . . . . . . . . . . . . . . . . . 25

2.11 Time evolution of the optical output power. . . . . . . . . . . . . 26

2.12 Agilent ADS schematic. The diode symbol shown represents the hierarchi-

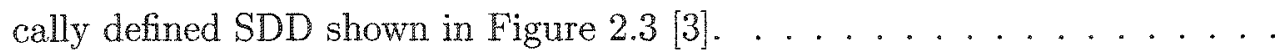

2.13 Laser modulation response curves. The laser bias current was swept from $20 \mathrm{~mA}$ to $100 \mathrm{~mA}[3] . \ldots \ldots \ldots . \ldots . \ldots . \ldots 29$

2.14 Peak modulation response $[3] \ldots \ldots \ldots \ldots$. . . . . . . . . . . . . . . 
2.15 Relaxation oscillation frequency and modulation bandwidth derived from Figure $2.13[3] . \ldots \ldots \ldots$. . . . . . . . . . . . . 30

3.1 The schematic symbol and port equations for a 3-port modified SDD including the temperature effects. . . . . . . . . . . . . . . . . . . . . 40

3.2 HP-ADS schematic diagram for the simulation setup. The diode symbol shown represents the hierarchically defined SDD shown in Figure 3.1. The output power is represented by a current in this simulation. . . . . . . 40

3.3 Threshold current against temperature as extracted from the model. Temperatures range from $250 \mathrm{~K}$ to $350 \mathrm{~K}$. The arrow is pointing to the threshold current value at room temperature. . . . . . . . . . . . . . . .

3.4 Optical gain as a function of temperature. Temperatures range from $250 \mathrm{~K}$ to $360 \mathrm{~K}$.

3.5 Time evolution of carrier density as a function of temperature. Temperature increased from $300 \mathrm{~K}$ to $380 \mathrm{~K}$ using steps of $5 \mathrm{~K}[2] \ldots \ldots . . . .$.

3.6 Time evolution of the optical power as a function of temperature. Temperature range from $300 \mathrm{~K}$ to $380 \mathrm{~K}$ using steps of $5 \mathrm{~K}[2] \ldots \ldots \ldots$. . . . .

3.7 Modulation bandwidth for different bias currents and different temperatures

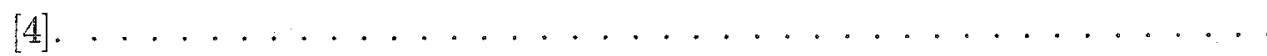

3.8 Laser relaxation oscillation frequency for different bias currents and different temperatures [4]. . . . . . . . . . . . . . . . . . . . 44

3.9 Schematic illustration of the hetrojunction leakage current $[5,6] \ldots \ldots 46$

3.10 Energy band diagram near the boundary of P-InP cladding layer and InGaAsP active layer. $E_{f c}$ and $E_{f v}$ are the electron and hole quasi-Fermi levels, $\Delta E_{c}$ and $\Delta E_{v}$ are the conduction and valence band barrier heights, and $E_{f v}^{\prime}$ and $E_{f v}$ are the hole quasi-Fermi levels in the p-cladding layer and active layer respectively $[5,6] \ldots \ldots \ldots 46$

3.11 Variation of the leakage current with temperature for different carrier densities. 48

3.12 Instantaneous leakage current and its variation with temperature. Note the rapid increase of the leakage current when $\mathrm{T}>330 \mathrm{~K} \ldots \ldots . \ldots 48$

3.13 Variation of the leakage current with carrier density. . . . . . . . . . . . 49

3.14 Variation of the leakage current with carrier density and the effect of temperature at lower temperatures from $250 \mathrm{~K} 310 \mathrm{~K} \ldots \ldots . \ldots 50$ 
3.15 Time evolution of the carrier density. Temperature varied between $250 \mathrm{~K}$ and $400 \mathrm{~K}$. Simulations were performed with and without including the effect of the leakage current $[7] \ldots \ldots \ldots \ldots \ldots$

3.16 Time evolution of the carrier density at temperatures $300 \mathrm{~K}, 330 \mathrm{~K}$ and 370 $\mathrm{K}$ with and without including the effect of the leakage current. . . . . . . 51

3.17 Time evolution of the optical power at temperatures $300 \mathrm{~K}, 330 \mathrm{~K}$ and 370 $\mathrm{K}$ with and without including the effect of the leakage current [2] . . . 52

3.18 Schematic symbol for the four port SDD model . . . . . . . . . . . . 56

3.19 Agilent-ADS schematic diagram for the simulation setup. The diode symbol shown represents the hierarchically defined SDD shown in Figure 3.18. The output power is represented by a current in this simulation. . . . . . . .

3.20 Time evolution of the carrier number with the effect of noise operators (solid lines) and without the effect of noise operators (doted lines) including tran-

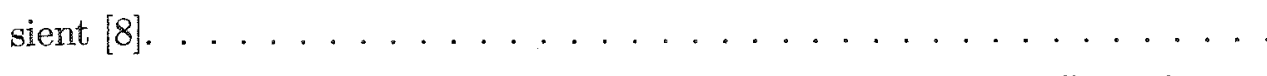

3.21 Time evolution of the carrier number with and without the effect of noise operators for steady state operation $[8] \ldots \ldots \ldots \ldots$

3.22 Time evolution of the photon number with the effect of noise operators (solid lines) and without the effect of noise operators (doted lines) including

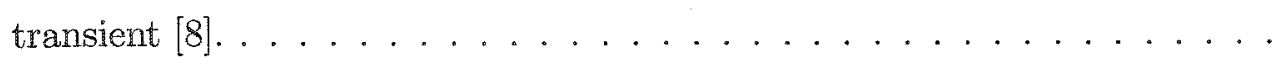

3.23 Time evolution of the photon number with and without the effect of noise operators for steady state operation $[8] \ldots \ldots \ldots$

3.24 Time evolution of the output power with the effect of noise operators (solid lines) and without the effect of noise operators (doted lines) including tran-

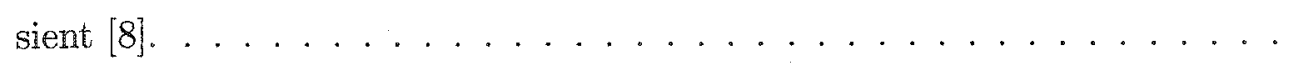

3.25 Time evolution of the output power with and without the effect of noise operators for steady state operation $[8] \ldots \ldots \ldots \ldots$

3.26 Laser diode relative intensity noise spectra (RIN) and frequency dependance of the amplitude noise $[4] . \ldots \ldots \ldots \ldots$

3.27 Intensity noise spectra at several power levels showing the shift of the peak frequency as the bias current increases. . . . . . . . . . . . .

3.28 Time evolution of the phase with the effect of noise operators (solid lines) and without the effect of noise operators (doted lines) including transient [8]. 64 
3.29 Time evolution of the phase with and without the effect of noise operators for steady state operation $[8] . \ldots \ldots \ldots . \ldots . \ldots 64$

3.30 Laser diode phase/frequency noise spectra $[4,8] \ldots \ldots \ldots 6$

4.1 Experimental setup for the DC measurements. . . . . . . . . . . . . 68

4.2 Output optical power versus driving current characteristics at room temperature for a $1.3 \mu \mathrm{m}$ InGaAsP-InP Fujitsu FLD3F7CZ butterfly packaged semiconductor laser. . . . . . . . . . . . . . . . . .

4.3 Experimental setup for the high frequency laser frequency response measurements. . . . . . . . . . . . . . . . . . .

4.4 Modulation response of a $1.3 \mu \mathrm{m}$ InGaAsP-InP (Fujitsu FLD3F7CZ) laser at bias levels of 10,20 , and $30 \mathrm{~mA} \ldots \ldots \ldots 70$

4.5 Experimental setup for the harmonic distortion measurements. . . . . . . 72

4.6 Measured power ratio of the second harmonic and the fundamental as a function of the bias current at carrier frequencies 0.5 and $1 \mathrm{GHz}$. The carrier power is $0 \mathrm{dBm} \ldots \ldots \ldots \ldots \ldots \ldots$

4.7 Measured power ratio of the second harmonic and the fundamental as a function of the bias current at carrier frequencies 0.5 and $1 \mathrm{GHz}$. The carrier power is $-5 \mathrm{dBm} . \ldots \ldots \ldots \ldots 73$

4.8 Experimental setup for intermodulation distortion measurements. . . . . . 74

4.9 Measured second order intermodulation distortion power as a function of the bias current. The carrier frequencies are $f_{1}=0.98$ and $f_{2}=1.02 \mathrm{GHz}$.

4.10 Measured higher $\left(2 f_{2}-f_{1}\right)$ third order intermodulation distortion as a function of the bias current. The carrier frequencies are $f_{1}=0.98$ and $f_{2}=1.02$ $\mathrm{GHz}$

4.11 Measured lower $\left(2 f_{1}-f_{2}\right)$ third order intermodulation distortion as a function of the bias current. The carrier frequencies are $f_{1}=0.98$ and $f_{2}=1.02$ $\mathrm{GHz} \ldots \ldots \ldots \ldots \ldots \ldots \ldots \ldots \ldots \ldots \ldots \ldots \ldots \ldots \ldots$

4.12 RIN measurement of FLD3F7CZ laser diode in the frequency range of 180 $\mathrm{MHz}$ to $10 \mathrm{GHz}$ at $10 \mathrm{~mA}\left(\simeq 1.25 I_{t h}\right)$ bias level using Agilent $71400 \mathrm{C}$ lightwave signal analyzer. The data window on top shows the measured values at the marker frequency $(2.61 \mathrm{GHz}) \ldots \ldots \ldots$ 
4.13 RIN measurement of FLD3F7CZ laser diode in the frequency range of 180 $\mathrm{MHz}$ to $10 \mathrm{GHz}$ at $10 \mathrm{~mA}\left(\simeq 1.5 I_{t h}\right)$ bias level at $(3.58 \mathrm{GHz})$. . . . . 78

4.14 RIN measurement of FLD3F7CZ laser diode in the frequency range of 180 $\mathrm{MHz}$ to $10 \mathrm{GHz}$ at $10 \mathrm{~mA}\left(\simeq 2 I_{t h}\right)$ bias level at $(5 \mathrm{GHz}) \ldots \ldots 78$

4.15 RIN measurement of FLD3F7CZ laser diode in the frequency range of 180 $\mathrm{MHz}$ to $10 \mathrm{GHz}$ at $10 \mathrm{~mA}\left(\simeq 3 I_{t h}\right)$ bias level at $(7.68 \mathrm{GHz}) . \ldots . . .79$

4.16 Experimental setup for laser linewidth measurements. . . . . . . . . 80

4.17 Laser linewidth measurement of FLD3F7CZ laser diode at $15.3 \mathrm{~mA}\left(\simeq 2 I_{\text {th }}\right.$ ) bias level using Agilent $71400 \mathrm{C}$ lightwave signal analyzer. The data window on top shows the measured values. . . . . . . . . . . . . . . . 81

4.18 Laser linewidth measurement of FLD3F7CZ laser diode at $23.4 \mathrm{~mA}\left(\simeq 3 I_{\text {th }}\right.$ ) bias level using Agilent $71400 \mathrm{C}$ lightwave signal analyzer. . . . . . . . .

4.19 Laser linewidth measurement of FLD3F7CZ laser diode at $60 \mathrm{~mA}$ bias level using Agilent $71400 \mathrm{C}$ lightwave signal analyzer. . . . . . . . . . . . 82

4.20 Simulation and measurement results of the output optical power versus bias current characteristics at $27 C$ for a $1.3 \mu \mathrm{m}$ InGaAsP-InP Fujitsu FLD3F7CZ butterfly packaged semiconductor laser. . . . . . . . . . . . .

4.21 Simulation and measurement results of the modulation frequency response for Fujitsu FLD3F7CZ laser at $10 \mathrm{~mA}$ bias levels . . . . . . . . . . .

4.22 Simulation and measurement results of the modulation frequency response for Fujitsu FLD3F7CZ laser at $20 \mathrm{~mA}$ bias levels [9].

4.23 Simulation and measurement results of the modulation frequency response for Fujitsu FLD3F7CZ laser at $30 \mathrm{~mA}$ bias levels. . . . . . . . . . . . .

4.24 Simulation and measurement of the second harmonic distortion as a function of the bias current at carrier frequencies 0.5 and $1 \mathrm{GHz}$ and power $0 \mathrm{dBm}[10] .87$

4.25 Simulation and measurement of the second harmonic distortion as a function of the bias current at carrier frequencies 0.5 and $1 \mathrm{GHz}$ and power $-5 \mathrm{dBm}$.

4.26 Measured and simulated second order intermodulation $\left(f_{1}+f_{2}\right)$ distortion power as a function of the bias current at carrier frequencies $f_{1}=0.98$ and $f_{2}=1.02 \mathrm{GHz} \ldots \ldots \ldots \ldots \ldots$ 
4.27 Measured and simulated lower $\left(2 f_{1}-f_{2}\right)$ third order intermodulation distortion components as a function of the bias current at carrier frequencies $f_{1}=0.98$ and $f_{2}=1.02 \mathrm{GHz} \ldots \ldots \ldots \ldots$

4.28 Measured and simulated higher $\left(2 f_{2}-f_{1}\right)$ third order intermodulation distortion components as a function of the bias current at carrier frequencies $f_{1}=0.98$ and $f_{2}=1.02 \mathrm{GHz} . \ldots \ldots \ldots . \ldots \ldots$

5.1 Optoelectronic feedback system. . . . . . . . . . . . . 93

5.2 Optical feed-forward system $[11] \ldots \ldots \ldots \ldots \ldots$

5.3 Phase shift modulation block diagram. . . . . . . . . . . . 95

5.4 Schematic diagram of the analog fibre-optic system employing a directly modulated semiconductor laser with external light injection. OC: refer to optical circulator, PC: polarization control, ECT-LD: external cavity tunable laser diode, and ATT: attenuator $[12] \ldots \ldots 96$

5.5 Schematic diagram for the cascaded linearized modulator [13] . . . . . . . 97

5.6 Optical domain post-distortion. . . . . . . . . . . . . 98

5.7 Electrical domain post-distortion. . . . . . . . . . . . . . . . . 98

5.8 Predistortion system. . . . . . . . . . . . . . . . . . . . . . . 99

5.9 Block diagram of the adaptive pre-distortion system. . . . . . . 100

6.1 A first order Volterra system representation of the pre-distorter. . . . . . . 109

6.2 A second order Volterra system representation of the pre-distorter. . . . . . 109

6.3 A third order Volterra system representation of the pre-distorter. . . . . . . 110

6.4 Entire block diagram of the the pre-distorter model represented by the Volterra system in time domain. . . . . . . . . . . . . . . . . . 110

6.5 Schematic diagram for the proposed adaptive pre-distortion circuit. . . . . 112

6.6 Block digram representation of the Volterra transfer functions representing the pre-distorter and laser models. . . . . . . . . . . . . . . . . . . 114

6.7 Simulation results for second harmonic distortion relative to carrier for Ortel $1510 \mathrm{~B}$ laser, with and without using the proposed pre-distorter based on CGF and MGF at $I_{b}=1.67 I_{t h}$ and $M D=0.14 \ldots \ldots \ldots$ 
6.8 Simulation results for second harmonic distortion relative to carrier for Fujitsu FLD3F7CZ and Ortel 1510B lasers, with and without using the proposed pre-distorter at $I_{b}=1.67 I_{t h}$ and $M D=0.14[14] . \ldots \ldots$. . . . .

6.9 Simulated second order intermodulation distortion (2IMD) for Ortel 1510B laser, with and without using the proposed pre-distorter based on CGF and MGF at $I_{b}=1.67 I_{t h}, \mathrm{MD}=0.14, \Delta f=10 \mathrm{MHz}[9] . \ldots \ldots$

6.10 Simulation results for second order intermodulation distortion (2IMD) relative to carrier for Fujitsu FLD3F7CZ and Ortel 1510B lasers, with and without using the proposed pre-distorter at $I_{b}=1.67 I_{t h}, \mathrm{MD}=0.14, \Delta f=$

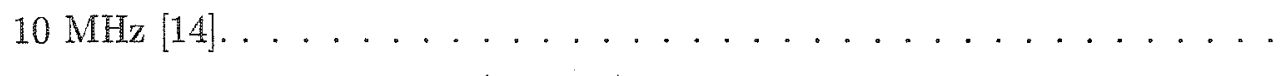

6.11 Simulation results for lower $\left(2 f_{1}-f_{2}\right)$ third order intermodulation distortion for Ortel $1510 \mathrm{~B}$ laser, with and without using the proposed pre-distorter at $I_{b}=1.67 I_{t h}, \mathrm{MD}=0.14, \Delta f=10 \mathrm{MHz} \ldots \ldots \ldots \ldots$

6.12 Simulation results for lower $\left(2 f_{1}-f_{2}\right)$ third order intermodulation distortion relative to carrier for Fujitsu FLD3F7CZ and Ortel 1510B lasers, with and without using the proposed pre-distorter at $I_{b}=1.67 I_{t h}, \mathrm{MD} 0.14, \Delta f=$ $10 \mathrm{MHz}[14] \ldots \ldots \ldots \ldots \ldots \ldots \ldots . \ldots \ldots$

6.13 Simulation results for three tones third order intermodulation distortion relative to carrier for Ortel $1510 \mathrm{~B}$ laser, with and without using the proposed pre-distorter at $I_{b}=1.67 I_{t h}, \mathrm{MD}=0.14, \Delta f=10 \mathrm{MHz}[9] . \ldots . .$.

6.14 Simulation results for three tones third order intermodulation distortion relative to carrier for Fujitsu FLD3F7CZ and Ortel 1510B lasers, with and without using the proposed pre-distorter at $I_{b}=1.67 I_{t h}, \mathrm{MD}=0.14, \Delta f=$ $10 \mathrm{MHz}[14] \ldots \ldots \ldots \ldots \ldots \ldots \ldots$

6.15 Simulation results for second harmonic distortion relative to carrier for Ortel $1510 \mathrm{~B}$ laser, with and without using the proposed pre-distorter at bias levels $I_{b}=1.25,1.75$, and $2.5 I_{\text {th }}$ and $\mathrm{MD}=0.14$.

6.16 Simulation results for second order intermodulation distortion (2IMD) relative to carrier for Ortel $1510 \mathrm{~B}$ laser, with and without using the proposed pre-distorter at bias levels $I_{b}=1.25,1.75$, and $2.5 I_{\text {th }}, \mathrm{MD}=0.14$ and $\Delta f=10 \mathrm{MHz} \ldots \ldots \ldots \ldots \ldots \ldots \ldots$ 
6.17 Simulation results for lower $\left(2 f_{1}-f_{2}\right)$ third order intermodulation distortion. for Ortel $1510 \mathrm{~B}$ laser, without and with using the proposed pre-distorter at bias levels $I_{b}=1.25,1.75$, and $2.5 I_{t h}, \mathrm{MD}=0.14$ and $\Delta f=10 \mathrm{MHz}[14] . \quad 134$

6.18 Simulation results for three tones third order intermodulation distortion relative to carrier for Ortel $1510 \mathrm{~B}$ lasers, with and without using the proposed pre-distorter at bias levels $I_{b}=1.25,1.75$, and $2.5 I_{t h}, \mathrm{MD}=0.14$ and $\Delta f=10 \mathrm{MHz} . \ldots \ldots \ldots \ldots$

6.19 Simulation results for second harmonic distortion relative to carrier for Fujitsu FLD3F7CZ laser, with and without using the proposed pre-distorter at bias levels $I_{b}=1.75$ and $2.5 I_{\text {th }}$ and $\mathrm{MD}=0.14 \ldots \ldots \ldots 135$

6.20 Simulation results for second order intermodulation distortion (2MD) relative to carrier for Fujitsu FLD3F7CZ laser, with and without using the proposed pre-distorter at bias levels $I_{b}=1.75$ and $2.5 I_{t h}$, MD0.14 and $\Delta f=10 \mathrm{MHz} . \ldots \ldots \ldots \ldots \ldots$

6.21 Simulation results for lower $\left(2 f_{1}-f_{2}\right)$ third order intermodulation distortion for Fujitsu FLD3F7CZ laser, with and without using the proposed predistorter at bias levels $I_{b}=1.75$ and $2.5 I_{t h}, \mathrm{MD}=0.14$ and $\Delta f=10 \mathrm{MHz}$ [14]. 136

6.22 Simulation results for three tones third order intermodulation distortion relative to carrier for Fujitsu FLD3F7CZ lasers, with and without using the proposed pre-distorter at bias levels $I_{b}=1.75$ and $2.5 I_{t h}, \mathrm{MD}=0.14$ and $\Delta f=10 \mathrm{MHz} \ldots \ldots \ldots \ldots \ldots \ldots \ldots$

B.1 Energy band diagram showing the band for net stimulated emission [15]. . 148

F.1 Direct expansion of the second order Volterra system. . . . . . . . 165

F.2 Direct expansion of the third order Volterra system. . . . . . . . . 165

G.1 Block diagram of the laser DC measurement setup. . . . . . . . . . 166

G.2 Block diagram of the laser frequency response measurement setup. . . . . . 167

G.3 Block diagram of the laser distortion measurement setup. . . . . . . . . 168 


\section{Chapter 1}

\section{Introduction}

\subsection{Introduction}

Recent analysis of eight-year trends in relative IT investment and relative prosperity between Europe and the USA [16] shows an increasing demand for multimedia communication or interactive services, which require some kind of broadband channel.

In practical scenarios of many of the present-day wireless communication networks, this channel includes a radio link connecting the customer and the base station, and coaxial cables between the base station and the central station.

A fibre optical link is recommended as the physical transmission medium between any network's base stations and central station to avoid the high attenuation of the coaxial cables in the radio frequency band. Fibre optics technology has a number of advantages that makes it attractive in a wide range of applications. Very low attenuation and large bandwidth are two major advantages.

The main reason we are seeking the analog transmission scheme is to bring the remote antenna signal back to a central office where it can be processed along with many other signals at low-cost and less complexity. The unique requirements of analog transmission over digital are for high signal to noise ratios at the receiver output, which necessitates high optical input power, and high end-to-end linearity to avoid distortion and prevent crosstalk between adjacent allocated bands. 


\subsection{Thesis motivation}

The potential for optical link improvements provides a compelling incentive for studying and analyzing laser diode performance. Accurate and rapid analysis and simulation of laser diode performance metrics such as modulation bandwidth [17-20], intensity and frequency noise levels $[18,21,22]$, and nonlinear distortion $[11,23-26]$ are required for overal] optical link system performance evaluation and network characterization. While sophisticated computer programs, such as those presented in [27-29], and numerous laser models such as those given in [30-33], can accurately predict important laser characteristics, they unfortunately have not yet found widespread acceptance in system level simulators due to their computational burden. Analytical solutions, numerical integration computer simulation models, and circuit models [21,34-40] have been introduced, but again they are not generally suitable for system simulations, either due to their computational burden or because of difficulties in incorporating these methods into a full optical link simulation.

The lack of laser linearity enhancement techniques is mainly due to the unavailability of a suitable laser models $[41,42]$ that include physical phenomena such as gain nonlinearities, gain saturation, index nonlinearities, thermal effects, leakage and noise. For this purpose, models suitable for system level simulations and which feature the above mentioned physical phenomena are required. Suitable linearization techniques and circuit implementations are required for analog optical transmission over fiber.

\subsection{Thesis objectives}

The main objective of this thesis is to enhance the performance of the analog optical transmitter for analog optical communications. This can be achieved through the following steps:

1. Review and analyze the available semiconductor laser models. 
2. Enhance the semiconductor laser diode models.

3. Predict the laser response and evaluate its performance using the proposed models.

4. Review and analyze the available linearization techniques.

5. Develop a suitable linearization technique.

6. Implement and test the proposed linearizer.

7. Evaluate laser diode performance with and without the linearizer.

\subsection{Thesis contributions}

The main contributions in this thesis have been reported in several publications and are summarized in the following.

- Enhancing the mathematical modeling of semiconductor laser diodes (emphasis is on $1.3 \mu \mathrm{m}$ GaAsInP-InP lasers).

- Implementation of a suitable model for system simulations. Emphasis is on MATLAB programming, SIMULINK and Agilent-ADS [1].

- Novel normalization technique to overcome the convergence difficulties [1].

- A novel enhanced normalized model, including simultaneous effects such as gain saturation, spectral hole burning, carrier transport, gain nonlinearities and its SDD implementation using Agilent-ADS [3].

- A novel analysis to calculate the thermal effects in the laser diode (outlined and included in the proposed model).

- Enhancing the laser model by adding the thermal effects and modifying the SDD implementation [2].

- Enhancing the laser model by adding the leakage current effects and modifying the SDD implementation [7]. 
- Introduction of a new analytical derivation for the laser noise correlation expressions and their simultaneous generation techniques $[8,43]$.

- Enhancing the above proposed model by adding the noise effects and modifying the SDD implementation [4].

- Prediction of the laser diode operation in the time and frequency domains, distortion levels [44] and CATV application.

- Model verification and parameters extraction $[10,14]$.

- Derivation of a new Volterra series expansion of the laser; derivation of a new inverse Volterra series expansion of the laser; proposal of a pre-distortion topology based upon derivations performed as components of the research [14].

- Software pre-distorter implementation and nonlinearity enhancement $[9,45]$.

\subsection{Thesis organization}

Laser diode modeling and gain modification is presented in Chapter 2, which also includes the technique of the normalization and simulation results in time and frequency domains. Chapter 3 presents the laser model modifications by including the temperature, leakage current and noise effects. Chapter 3 also includes the simulation results in each case. Model verification and validation based on the comparison between simulation and measurement results are presented in Chapter 4 . In Chapter 5, a review of the laser nonlinearity and different linearization techniques is presented. Development of the pre-distorter model, pre-distorter implementation and the nonlinearity performance evaluation with and without using the pre-distorter are discussed in Chapter 6. Finally, Chapter 7 summarizes the results arising from this work, provides the overall conclusions, and presents recommendations for future research. 


\section{Chapter 2}

\section{Semiconductor-Diode Laser Modeling}

In this chapter, general introduction for the semiconductor laser operation as well as a review and analysis of the published laser diode models are presented. This review is expanded by modifying the rate equations governing the dynamics of the laser diode theory adding the simultaneous effects of different physical phenomena such as index non-linearity and the nonlinear gain effect leading to the modified gain formulation. Furthermore, suitable models for system level simulations based on the modified rate equations are implemented using Agilent-ADS simulation tool. Transient, small signal and large signal simulation results in time and frequency domains are demonstrated.

\subsection{Historical background}

The earliest semiconductor diode lasers were implemented using $\mathrm{p}-\mathrm{n}$ homo-junction formed in crystalline blocks of gallium arsenide (GaAs) or Gallium Phosphide Arsenide $G a P_{x} A s_{1-x}$ $[6,46]$. As early as 1963 , it was suggested that semiconductor lasers might be improved if a layer of one semiconductor material were sandwiched between two cladding layers of another semiconductor that has a relatively wider band gab. Such a device is called hetero-structure laser [6]. Single hetero-structure lasers were developed next, where the active region had on one side a cladding layer of higher band gab and lower refractive index material $[6,46]$. The motivation for developing the single hetero-structure is that carriers rapidly diffuse away from the junction in the conventional $\mathrm{p}-\mathrm{n}$ junction. To compensate for 
this diffusion, injected currents of tens of thousands of ampere per square centimeters are required. Further reduction of the threshold currents can be achieved by using the double hetero-junction (or broad-area laser) such as $G a_{x} A l_{1-x} A s-G a A s-G a_{x} A l_{1-x} A s$ sandwich. This type of device allows CW operation at room temperature with current densities around $1000 \mathrm{~A} / \mathrm{cm}^{2}$. The theory of operation behind this type of laser device is to confine the optical mode by varying the index of refraction laterally, and for this reason they are called index-guided lasers. Long wavelength semiconductor lasers in the range 1.1-1.6 $\mathrm{mm}$ are of considerable interest for optical fibre communication $[6,47]$. In the early 1970 's the combination of Indium Gallium Arsenide Phosphide-Indium Phosphide (InGaAsP-InP) turned out to be the most suitable in view of the fact that it is nearly perfect lattice matched $[6,47]$. The active layer is composed of $I n_{1-x} G a_{x} A s_{y} P_{1-y}$ quaternary alloy. By varying the mole fractions $x$ and $y$, almost any wavelength in the 1.1-1.6 $\mu \mathrm{m}$ range can be selected. In 1977-1980 1.3 $\mu \mathrm{m}$ InGaAsP-InP lasers were introduced to benefit from the low-loss dispersion free fibers at $1.3 \mu \mathrm{m}$ and ultra-low-loss $(\approx 0.2 \mathrm{~dB} / \mathrm{Km})$ at $1.55 \mu \mathrm{m}$ wavelength [6]. Following this the theory of InGaAsP lasers operating in the wavelength range $1.3 \mu \mathrm{m}-1.6 \mu \mathrm{m}$ proceeded at an enormous pace [6]. The primary motivation was their application in optical fiber communication in which laser size are in the same order of the optical fiber core size, which makes the coupling more efficient.

\subsection{Basic principles of semiconductor lasers}

The operation of most lasers can be described by the electron transitions, which may occur between two energy levels of a hypothetical atomic system. Laser action is the result of three key processes: photon absorption, spontaneous emission, and stimulated emission. These three processes are represented by the simple two-energy level diagram as shown in Figure 2.1 [48]. In this diagram $E_{1}$ is the ground state (or lower energy level) and $E_{2}$ is 


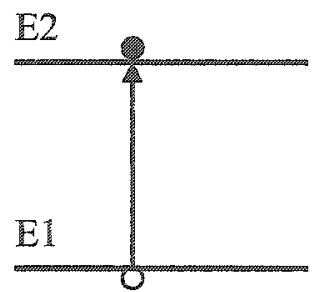

(a) Absorption

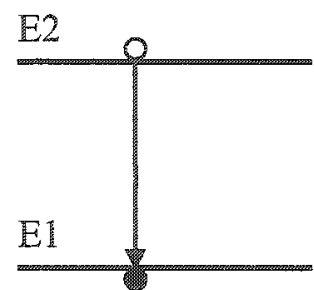

(b) Spontaneous emission

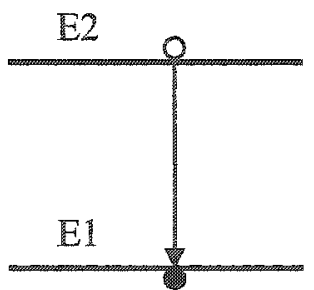

(c) Stimulated emission

Figure 2.1: Two-energy-level diagrams

the excited state (or higher energy level). According to Planck's law, a transition between these two states involves the absorption or emission of a photon energy $h f_{12}=E_{2}-E_{1}$. When a photon of energy $h f_{12}$ impinges on the system, an electron in state $E_{1}$ can absorb the photon energy and be excited to state $E_{2}$, as shown in Figure 2.1(a). Since $E_{2}$ is an unstable state, the electron will shortly return to the ground state as shown in Figure 2.1(b), thereby emitting a photon of energy $h f_{12}$. This is called spontaneous emission and it occurs without any external stimulation. It is also isotropic and has random phase. The electron can also be induced to make a downward transition from the excited level to the ground level by an external stimulation as shown in Figure 2.1(c). If a photon of energy $h f_{12}$ impinges on the system while the electron is still in its excited state, the electron is immediately stimulated to drop to the ground state and give off a photon of energy $h f_{12}$. The remarkable feature is that the emitted photon matches the original photon not only in its wavelength but also in direction of propagation. This means that stimulated process results in coherent radiation as the waves associated with the stimulating and stimulated photons have identical frequencies are in phase and have the same state of polarization. The resultant emission is known as stimulated emission. In thermal equilibrium the density of excited electrons is very small. Most of the photons incident on the system will therefore be absorbed. In this state, the density of electrons in the lower energy level is more than the density of the electrons in the upper energy level. In this case, stimulated emission is 
essentially negligible. The amplification or lasing condition in which stimulated emission exceeds the absorption only happens when the population of the excited states is greater than that of the ground states. This condition is known as population inversion. Since this is not an equilibrium condition, population inversion is achieved by various pumping techniques.

\subsection{Semiconductor laser modeling}

There are two basic classical methods of modelling mathematically the operation of semiconductor lasers. The first method applies the concept of photon/electron particle exchange. This is the standard rate-equation approach which is robust and well researched $[15,46,49]$ but can be difficult to apply when there are strong non-uniformities in the laser's materials. In this method, spontaneous emission which initiates lasing, optical gain which is essential to achieve lasing, and other processes involved in lasing all use quantum processes at the level of single atoms and electrons within the lasing material [46]. The second method is based on the interactions between electromagnetic field and the electric dipoles in an active optical medium. This method is effective when the refractive index is nonlinear and changes inside the laser, leading to permittivity changes. Phenomena causing the gain nonlinearities such as spatial hole burning $[6,50,51]$, carrier heating $[52-58]$ and gain saturation [58-60], coupled with the effect of the leakage current and noise, significantly affect the performance of semiconductor lasers. Therefore, they should be simultaneously included in the mathematical modeling of the semiconductor laser to accurately predict its response.

Accurate and rapid analysis and simulation of laser diode performance metrics such as modulation bandwidth [17-20], intensity and frequency noise levels [18,21,22], and nonlinear distortion $[11,23-26]$ are required for overall optical link system performance evaluation and network characterization. 
Models based on simplified rate equations [61-63] are based on assumptions that make them unsuitable for simulation of analog signal transmission. They also limit the possibility of enhancing optical link parameters such as the dynamic range. Sophisticated computer programs, such as those based on the solution of large sets of simultaneous equations [64-66] have been presented in [27-29]. As well, numerous laser models such as those given in [30-33] have been developed in order to predict some of the important laser characteristics. However, these models didn't include most of the physical effects and failed to gain widespread acceptance in system level simulators due to their computational complexity. Other approaches that are based on numerical analysis and computer simulation models, and circuit based models $[6,21,34-40,67]$ have been introduced, but were deemed to be not suitable for general system simulations either due to their computational burden or because of difficulties in incorporating these models into a full optical link simulators. Because of the complexity of representing the semiconductor lasers as a homogenously broadened laser [68], the energy levels representation of the laser model was a strong candidate to illustrate the methodology of converting the electric field into photons. Thus, in this thesis, the semiclassical theory is used to describe the interactions between the electromagnetic field variation using the classical theory and the electric dipoles perturbation energy in the density matrix using quantum mechanics in an active optical medium. This method is used to derive the modified gain formulation and to describe the electric field phase variation inside the active medium. The theory of quantum mechanics is used to derive the laser rate equations by coupling the atomic system to the field system and by adding the electric-dipole perturbation energy to the Hamiltonian [69] using the density matrix method [70]. 


\subsection{Conventional semiconductor laser rate equations}

Rate equations form a commonly accepted approach for modeling the semiconductor lasers and simulating the dynamics of the electron and photon population. This is due to its simplicity, clarity and ease of modifying the applicability extension $[6,46,48,71-73]$ of the rate equations. The main approaches used for the derivation of the rate equations are; (1) The classical wave theory approach $[6,15,46,70]$, and (2) The combination of quantum mechanics and density matrix methods $[15,69,74-80]$. Simplified single mode rate equations with simplifed linear gain formulation can be expressed as follows $[6,20,30,61-63,81-91]$,

$$
\begin{aligned}
& \frac{d \mathbb{P}(t)}{d t}=\Gamma G \mathbb{P}(t)+\Gamma \beta_{s p} \frac{\mathbb{C}(t)}{\tau_{e}}-\frac{\mathbb{P}(t)}{\tau_{p h}} \\
& \frac{d \mathbb{C}(t)}{d t}=\frac{I(t)}{q V_{c}}-G \mathbb{P}(t)-\frac{\mathbb{C}(t)}{\tau_{e}}
\end{aligned}
$$

in which $\mathbb{P}$ represents the intracavity photon density, $\mathbb{C}$ is the active region carrier density, $\Gamma$ is the mode confinement factor (or the filling factor), $\beta_{s p}$ is the spontaneous emission factor, $\tau_{e}$ is the electron life time, $\tau_{p h}$ is the photon life time, $I(t)$ is the injected current, $q$ is the electronic charge and $V_{c}$ is the volume of the active region.

The gain, $G$, is expressed in one of the forms given by equation 2.5.1a-d.

The system described by equations (2.4.1a) and (2.4.1b) includes the basic set of rate equations which mathematically describe the interaction between electrons and photons inside the active region.

Equation (2.4.1a), represents the rate of change of the photon density inside the laser cavity. The first term of the right hand side, $\Gamma G \mathbb{P}(t)$, represents the rate of stimulated emission of photons by electrons within the laser cavity that can be considered as the source of photons. The next term, $\beta_{s p} \Gamma \mathbb{C}(t) / \tau_{e}$, is the rate of spontaneous emission of photons due to the recombined electrons and photons. The last term, $-\mathbb{P}(t) / \tau_{p h}$, corresponds to the decay rate of the number of photons due to losses. 
Equation (2. A.1b) represents the rate of change of the electrons density. In the right hand side of $(2.4 .1 \mathrm{~b})$ the first term, $I(t) / q V_{c}$, represents the rate of electrons pushed into the conduction band by the effect of the driving current, $I(t)$. This means that increasing the current flow leads to higher electron concentration in the conduction band. The next term, $-G \mathbb{P}(t)$, corresponds to the removal of electrons from the interaction region caused by the stimulated recombination associated with the material optical gain. The last term, $-\mathbb{C}(t) / \tau_{e}$, represents the net electrons decay rate due to the spontaneous recombination.

\subsection{Modified semiconductor laser rate equations}

Results of experimental measurements reported in [32,90,92-99], and device simulation in $[33,65,89,100-110]$ demonstrated the limitations of the simplified laser rate equations. Therefore, the simplified system of rate equations need to be modified to extend its applicability while still maintaining its clarity and physical interpretation. One of the important modifications of the simplified rate equations involves a new derivation of the gain parameter. These modifications are described in the following.

\subsubsection{Modified semiconductor laser gain formulation}

The difference between the analytical and experimental results reported in literature showed the inadequacy of modeling the laser using rate equations with linear gain formulation $[6,69,76]$. Gain formulas appearing in the literature $[6,81]$ such as the empirically obtained linear gain formula given by equation (2.5.1a) represent small signal gain and does not account for most of the laser media nonlinearities. The modified nonlinear versions of (2.5.1a) given by equations (2.5.1b)- (2.5.1d) and which appeared as examples in $[20,61-63,82-91,111]$ were either derived by treating the laser diode as a two-level system, or were empirically obtained by an experimental fitting of one or more parameters. These modified gain formulations either provide small signal gain which is valid for small 
power [82-84], or yield mathematically complicated gain equations that are difficult to include in dynamic models of semiconductor lasers [77,112]. However, these nonlinear gain formulations provide in some cases and under specific conditions close agreement between the theoretical and experimental results. These gain formulas can not be considered as general gain expressions since they are expressed in terms of fitting parameters that are related to particular measured quantities. These fitting parameters do not directly describe the cause of the gain nonlinearity, nor include the parameters reflecting the influence of a specific nonlinear phenomenon on the gain formulation. Expressions for the gain, G, that appeared in the literature are given by the following,

$$
\begin{aligned}
\mathrm{G} & =v_{g} \wp\left(\mathbb{C}-\mathbb{C}_{t r}\right) \\
\mathrm{G} & =v_{g} \wp\left(\mathbb{C}-\mathbb{C}_{t r}\right)(1-\aleph \mathbb{P}) \\
\mathrm{G} & =v_{g} \wp\left(\mathbb{C}-\mathbb{C}_{t r}\right) /(1+\aleph \mathbb{P}) \\
\mathrm{G} & =v_{g} \wp\left(\mathbb{C}-\mathbb{C}_{t r}-\mathbb{C} \beta \frac{\Delta T}{\Delta T_{l}}\right)
\end{aligned}
$$

In the above equations, $\wp$ is the differential gain coefficient, $\mathcal{N}$ is the gain suppression factor, $v_{g}$ is the group velocity, $\mathbb{C}_{t r}$ is the transparency carrier density corresponding to the onset of population inversion, $\Delta T$ is the deviation of carrier temperature from the corresponding temperature of the lattice, $T_{l}$, and $\beta$ is a fitting parameter. The referenced gain formulas were derived by treating the laser diode as a two-level system or by using the third order perturbation theory. Both methodologies do not include the intraband transitions and associated effects. The use of the density matrix approach $[6,69,75-77]$ can correctly include the intraband transition effects. A modified optical gain expression including the effects of spectral hole burning, index of refraction nonlinearities, and gain saturation is given by Equation (2.5.2) [3] (see Appendix A for the details of the derivation).

$$
G(\mathbb{C}, \mathbb{P})=\frac{\Gamma \frac{c}{n_{g}} \wp\left(\mathbb{C}-\mathbb{C}_{t r}\right)}{\sqrt{1+\left(\Gamma \omega_{o} \hbar /\left(\varepsilon_{o} \bar{n} n_{g} I_{s}\right)\right) \mathbb{P}}}
$$


In the above equation, $\bar{n}$ and $n_{g}$ represent the effective mode index and the group refractive index respectively, $\hbar=h / 2 \pi$ (Planck's constant), $I_{s}$ is the saturation intensity and $c$ is the free space light speed. Both the modified gain formula (MGF) given by (2.5.2) and the conventional gain formula (CGF) given by (2.5.1c) were used in the simulation of important laser characteristics. Comparison ${ }^{1}$ between the simulation and measurement results $[9,113]$ of some of the laser characteristics such as the modulation responses confirms that the modified gain formulation behaves in accordance with the measurement results, while the simulated results obtained from the conventional gain formulations do not.

\subsubsection{Semiconductor laser model with modified gain}

Assuming $\mathbb{P}_{s}=\left(\varepsilon_{o} \bar{n} n_{g} / \Gamma \hbar \omega\right) I_{s}$, the modified gain formulation given in $(2.5 .2)$ can be simplified and given by the following equation:

$$
G(\mathbb{C}, \mathbb{P})=\frac{G_{n}\left(\mathbb{C}-\mathbb{C}_{t r}\right)}{\sqrt{1+\mathbb{P} / \mathbb{P}_{s}}}
$$

in which $\mathbb{P}_{s}$ represents the saturation power, $G_{n}=\Gamma v_{g} \wp$, and $v_{g}=c / n_{g}$. The derived simplified gain formula given by equation (2.5.3) agrees with the empirically obtained linear gain formula that has been introduced in $[6,114]$. Using the modified gain formulation (2.5.3) and equations (2.4.1a) and (2.4.1b) leads to the modified laser rate equations given by,

$$
\begin{aligned}
& \frac{d \mathbb{P}(t)}{d t}=\frac{G_{n}\left(\mathbb{C}-\mathbb{C}_{t r}\right)}{\sqrt{1+\mathbb{P}(t) / \mathbb{P}_{s}}} \mathbb{P}(t)-\frac{G_{n}\left(\mathbb{C}_{t h}-\mathbb{C}_{t r}\right)}{\sqrt{1+\mathbb{P}(t) / \mathbb{P}_{s}}} \mathbb{P}(t)+\Re_{s p} \\
& \frac{d \mathbb{C}(t)}{d t}=\frac{I(t)}{q V_{c}}-\frac{G_{n}\left(\mathbb{C}-\mathbb{C}_{t r}\right)}{\Gamma \sqrt{1+\mathbb{P}(t) / \mathbb{P}_{s}}} \mathbb{P}(t)-\frac{\mathbb{C}(t)}{\tau_{e}}
\end{aligned}
$$

in which $\mathbb{C}_{i h}$ is the threshold carrier density, and $\Re_{s p}=\Gamma \beta_{s p} \eta_{s p} \mathbb{C} / \tau_{e}$ is the spontaneous emission rate in which $\eta_{s p}=B \mathbb{C} \tau_{e}$ is the internal spontaneous quantum efficiency, and $B$ is the radiative recombination coefficient. In practice, $\beta_{s p}$ is treated as a fitting parameter, typically in the range of $10^{-4}-10^{-5}$ for GaAs [115] and InGaAsP lasers [6, 114].

\footnotetext{
${ }^{1}$ The measured results in [113] are for different laser parameters than in $[6,61]$.
} 


\subsubsection{Threshold lasing condition and steady state solution}

The threshold current can be calculated by solving equations (2.5.4) and (2.5.5) in steady state.

As shown in Appendix A, the threshold condition is given by,

$$
g_{t h}=\frac{\alpha_{i n t}+\alpha_{m}}{\Gamma}
$$

in which $\alpha_{i n t}$ and $\alpha_{m}$ represent the internal and external (facet) losses and given by,

$$
\begin{aligned}
\alpha_{i n t} & =\frac{k_{o} \sigma}{\bar{n} \varepsilon_{o} \omega} \\
\alpha_{m} & =\frac{1}{2 L} \ln \left(\frac{1}{R_{1} R_{2}}\right)
\end{aligned}
$$

Using equation (2.5.6), the solution of (2.5.4) in steady state will lead to,

$$
\Gamma v_{g} \wp\left(\mathbb{C}_{t h}-\mathbb{C}_{t r}\right)=v_{g}\left(\alpha_{m}+\alpha_{i n t}\right)
$$

from which, the threshold carrier density $\mathbb{C}_{t h}$ can be derived and given as follows,

$$
\mathbb{C}_{t h}=\frac{\left(\alpha_{m}+\alpha_{i n t}\right)+\Gamma_{\wp} \mathbb{C}_{t r}}{\Gamma_{\wp}}
$$

In this solution, the carriers and photons decay rates have been expressed as follows $[6,15$, $46,69]$,

$$
\begin{aligned}
\tau_{e}^{-1} & =B \mathbb{C}+A_{n r}+C_{a} \mathbb{C}^{2} \\
\tau_{p h}^{-1} & =v_{g}\left(\alpha_{m}+\alpha_{i n t}\right)
\end{aligned}
$$

To solve equation (2.5.5) in steady state, we will assume that $\Re_{s p}=0$.

The threshold current can be derived from the solution of equation (2.5.5) in steady state and is given as,

$$
I_{t h}=\frac{q V_{c} \mathbb{C}_{t h}}{\tau_{e}\left(\mathbb{C}_{t h}\right)}
$$




\subsection{Laser model implementation}

\subsubsection{Semiconductor laser diode modeling using STMULINK}

Different methods for the laser model implementation have been discussed [1], based on which the the laser model was simulated using SIMULINK. Numerical solution based on the modified Ronsenbrock formula of order two [116] successfully converged to the laser rate equation solutions. Other numerical solutions experienced convergence difficulty, particularly in the laser threshold region. The block diagram shown in Figure 2.2 shows the semiconductor laser rate equations implemented in SIMULINK

Due to the Convergence problems, significant simulation time was required. Furthermore,

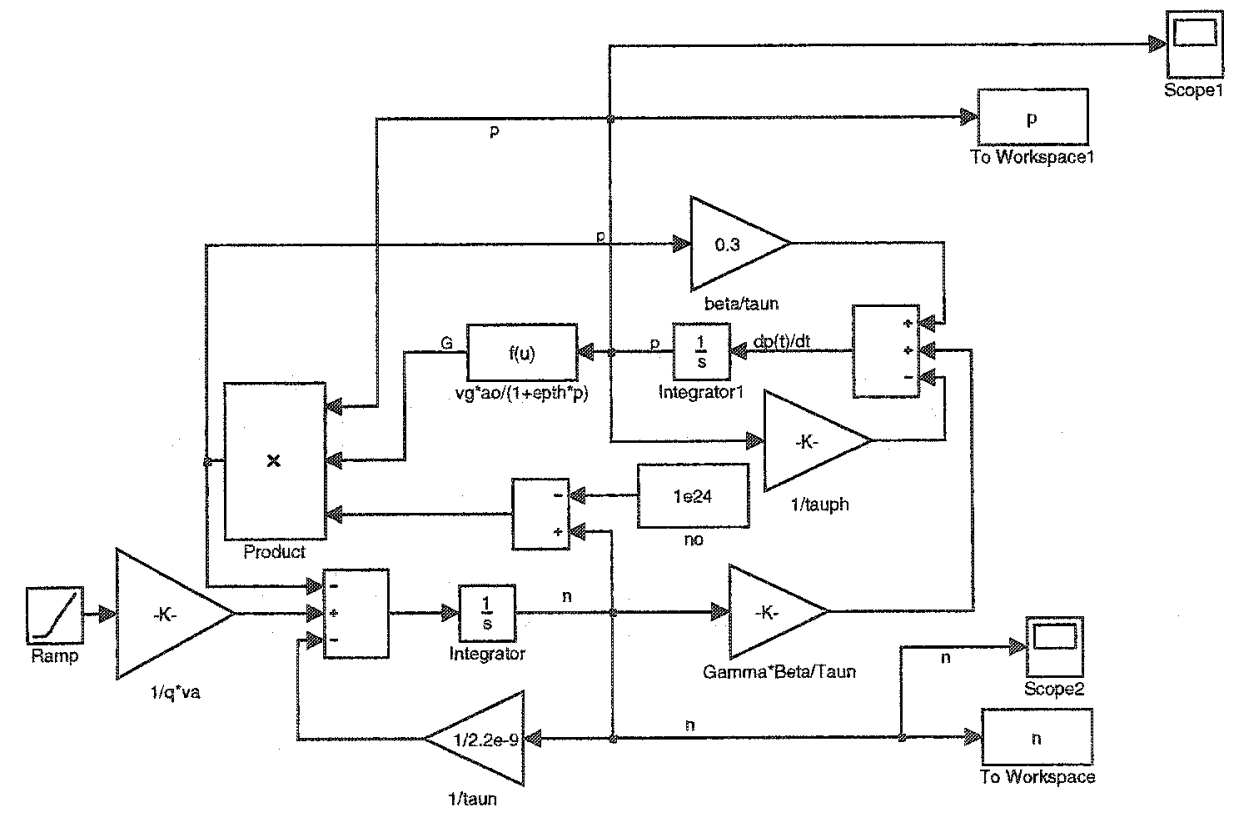

Figure 2.2: SIMULINK block diagram representation of semiconductor laser rate equations. [1]

it is not possible to obtain frequency domain simulations using SMULINK for different laser models. Hence SIMULINK is not deemed suitable for small signal and large signal simulations in both time and frequency domains. 


\subsubsection{Normalized rate equations}

The numerical method used to solve the laser rate equations must converge over the input signal domain, including the laser threshold region. Normalized rate equations enhance the convergence of numerical solvers and are suitable for transient, small signal, and large signal analysis.

\subsubsection{Normalized photon rate equation}

Simplifying equation (2.5.4) and multiplying both sides by $\tau_{e} / \Gamma \mathbb{C}_{t h}$ leads to,

$$
\frac{d\left(\frac{\tau_{e}}{\Gamma \mathbb{C}_{t h}} \mathbb{P}(t)\right)}{d t}=\frac{\tau_{e} G_{n}}{\Gamma} \frac{\left(\frac{\mathbb{C}}{\mathbb{C}_{t h}}-\frac{\mathbb{C}_{t r}}{\mathbb{C}_{t h}}\right)}{\sqrt{1+\mathbb{P}(t) / \mathbb{P}_{s}}} \mathbb{P}(t)-\left(\frac{\tau_{e}}{\Gamma \mathbb{C}_{t h} \tau_{p h}}\right) \mathbb{P}(t)+\beta_{s p} \frac{\mathbb{C}}{\mathbb{C}_{t h}}
$$

using (2.5.12) and (2.5.9) yield,

$$
\tau_{p h} G_{n}=\frac{1}{\mathbb{C}_{t h}\left(1-\mathbb{C}_{t r}^{n}\right)}
$$

using (2.6.2) in (2.6.1) to simplify the results yields the following normalized photon rate equation.

$$
\frac{d \mathbb{P}_{n}(t)}{d t}=\frac{\left(\mathbb{C}_{n}-\mathbb{C}_{t r}^{n}\right)}{\tau_{p h}\left(1-\mathbb{C}_{t r}^{n}\right)} \frac{\mathbb{P}_{n}}{\sqrt{1+\mathbb{P}_{r}^{n}}}-\frac{\mathbb{P}_{n}}{\tau_{p h}}+\beta_{s p} \mathbb{C}_{n}
$$

in which we assume that the internal quantum efficiency $\eta_{s p}$ equals $100 \%, \mathbb{P}_{n}=\tau_{e} \mathbb{P} /\left(\Gamma \mathbb{C}_{t h}\right)$, $\mathbb{C}_{n}=\mathbb{C} / \mathbb{C}_{t h}, \mathbb{C}_{t r}^{n}=\mathbb{C}_{t r} / \mathbb{C}_{t h}, \mathbb{P}_{r}^{n}=\mathbb{P}_{n} / \mathbb{P}_{s}^{n}$, and $\mathbb{P}_{s}^{n}=\tau_{e} \mathbb{P}_{s} / \Gamma \mathbb{C}_{t h}$

\subsubsection{Normalized electron rate equation}

Multiplying both sides of (2.5.5) by $\tau_{e} / \mathbb{C}_{t h}$ and using (2.6.2) leads to the normalized electron $^{1}$ rate equation given by,

$$
\frac{d \mathbb{C}_{n}(t)}{d t}=\frac{I(t)}{q V_{c} \mathbb{C}_{t h}}-\frac{\left(\mathbb{C}_{n}-\mathbb{C}_{t r}^{n}\right)}{\tau_{e} \tau_{p h}\left(1-\mathbb{C}_{t r}^{n}\right)} \frac{\mathbb{P}_{n}}{\sqrt{1+\mathbb{P}_{r}^{n}}}-\frac{\mathbb{C}_{n}}{\tau_{e}}
$$

\footnotetext{
${ }^{1}$ Under electroneutrality condition, the word electrons and carriers are identical [114].
} 


\subsubsection{Symbolically defined laser diode models}

Agilent-ADS (Agilent Advanced Design Systems) was chosen for implementation of the laser models because it is suitable for simulations from the circuit level up to the system level. It also offers both time-domain and frequency domain simulation and modeling capabilities. The semiconductor laser models defined by equations (2.6.3) - (2.6.4) were implemented using a 3-port Symbolically Defined Devices (SDDs) in Agilent-ADS. The laser rate equations were implemented as a combination of implicit and explicit part relationships, as shown in Figure 2.3. $\mathbb{P}_{n}(t)$ is represented by the "current" flowing into port 2 and is defined by the implicit function $F[2,0]$. Similarly $\mathbb{C}_{n}(\mathrm{t})$ is represented by the "current" flowing into port 3 and is defined by the implicit function $\mathrm{F}[3,0] . I(t)$ is represented by the "current" flowing into port 1 and is defined by explicit function $\mathbb{1}[1,0]$. The derivative $d \mathbb{P}_{n}(t) / d t$ is related to the current flowing out of port 2 by the explicit relation $F[2,1]$. The derivative $d \mathbb{C}_{n}(t) / d t$ is related to the current flowing out of port 3 by the explicit relation $\mathrm{F}[3,1]$. The current controlled voltage source (CCVS) shown in the schematic diagram is used to de-normalize the normalized photon density $\mathbb{P}_{n}(t)$ into the actual intracavity photon density by multiplying it by $\Gamma \mathbb{C}_{t h} / \tau_{e}$. The power emitted by each facet $\left(P_{\text {out }}^{1}\right.$ and $\left.P_{o u t}^{2}\right)$ is related to the photon density by equation $(2.6 .5)[6,15,73]$.

$$
\begin{aligned}
& P_{o u t}^{1}=\frac{\left(1-R_{1}\right) \sqrt{R_{2}}}{\left(\sqrt{R_{1}}+\sqrt{R_{2}}\right)\left(1-\sqrt{R_{1} R_{2}}\right)} \frac{\mathbb{P}}{\tau_{p h}} V_{c} \frac{\alpha_{m}}{\left(\alpha_{m}+\alpha_{i n t}\right)} E \\
& P_{o u t}^{2}=\frac{\left(1-R_{2}\right) \sqrt{R_{1}}}{\left(\sqrt{R_{1}}+\sqrt{R_{2}}\right)\left(1-\sqrt{R_{1} R_{2}}\right)} \frac{\mathbb{P}}{\tau_{p h}} V_{c} \frac{\alpha_{m}}{\left(\alpha_{m}+\alpha_{i n t}\right)} E
\end{aligned}
$$

In the conversion process of photons to optical power we assumed that the facet reflectivities are identical, that $100 \%$ of the injected carriers will recombine radiatively and generate photons, and that the photon generation rate $\tau_{p h}^{-1}$ is balanced with the photon escape rate $v_{g} \alpha_{m}$ (rate of photons escape out of the laser cavity) to preserve the steady state. 
Applying these assumptions on equation (2.6.5), using (2.5.12) and replacing $E$ by $h f$ leads to the following equation:

$$
P_{\text {out }}=\frac{1}{2}\left(v_{g} \alpha_{m}\right)\left(\mathbb{P} V_{c}\right)(h f)
$$

\subsection{Simulation results}

The three-port SDD laser model shown in Figure 2.3 is used as part of an Agilent-ADS schematic diagram to predict the different semiconductor laser characteristics. Simulation setup shown in Figure 2.4 is used to predict the semiconductor laser characteristics in the time domain. The simulation setup suitable for the prediction of the semiconductor laser characteristics in the frequency domain is presented in section 2.7.3.

\subsubsection{Time domain steady state simulation results}

The proposed model shown in Figure 2.3 was used in the simulation setup shown in Figure 2.4 to predict the steady state and dynamic characteristics of the laser diodes using realistic parameters for InGaAsP-InP semiconductor lasers, such as those shown in Table 2.1 which was extracted from $[6,21,117]$.

\subsubsection{Parameters extraction}

The parameter extraction methodology is very important for successful model verification. The ideal case for parameter extraction where a unique measurement for each parameter exists [118]. Measuring these parameters is not an easy task and needs many extensive equipments. Using the parameters extraction algorithm presented in Appendix $\mathrm{G}$ and the data given in Table 2.1, the proposed model is able to predicts most of the laser parameters such as those given in Table (2.2). Note that $G_{t h o}$ is the threshold gain at 


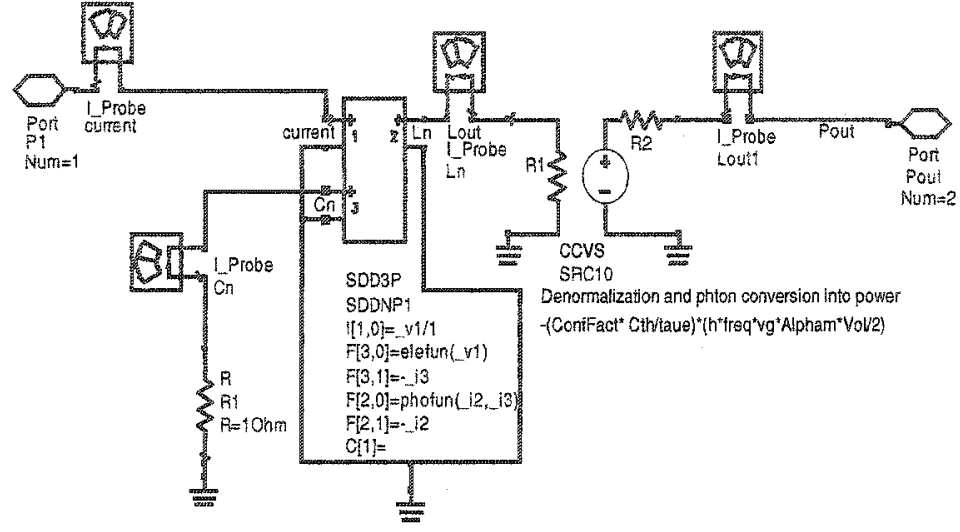

Parameters, Constants, and Calculations

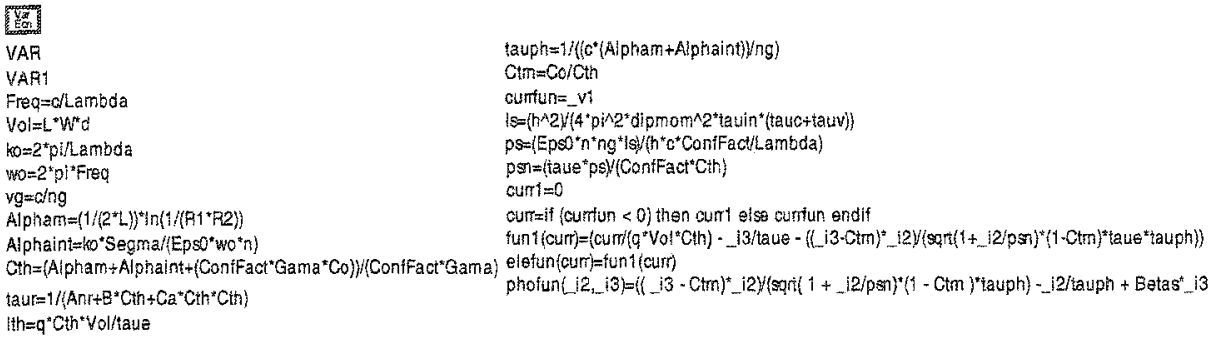

Figure 2.3: The schematic symbol and port equations for a 3-port SDD [2].
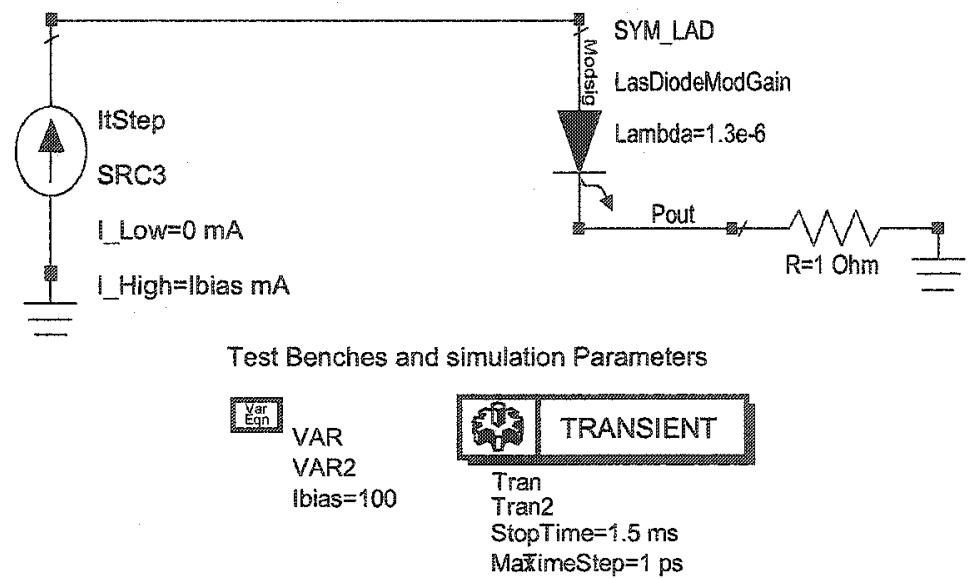

Figure 2.4: Agilent-ADS schematic diagram for the simulation setup. The diode symbol shown represents the hierarchically defined SDD shown in Figure 2.3. The output power is represented by a current in this simulation. 
Table 2.1: Device Parameters for InGaAsP/InP semiconductor laser diodes

\begin{tabular}{||l|l||}
\hline \multicolumn{1}{|c|}{ Parameter definition } & \multicolumn{1}{|c||}{ Symbol \& Value } \\
\hline \hline Active region length & $\mathrm{L}=250 \mu \mathrm{m}$ \\
Active region width & $\mathrm{W}=2 \mu \mathrm{m}$ \\
Active region thickness & $\mathrm{d}=0.2 \mu \mathrm{m}$ \\
Operating wave length & $\lambda=1.3 \mu \mathrm{m}$ \\
Transparency carrier density & $\mathbb{C}_{t r o}=1 \times 10^{24} \mathrm{~m}^{-3}$ \\
Reflectivity of Facets & $\mathrm{R} 1=\mathrm{R} 2=0.3246$ \\
Mode confinement factor & $\Gamma=0.3$ \\
Effective mode index & $\bar{n}=3.5$ \\
Group refractive index & $n_{g}=4$ \\
Line width enhancement factor & $\beta=5$ \\
Differential gain coefficient & $\wp=2.5 \times 10^{-20} \mathrm{~m}^{2}$ \\
Nonradiative recombination rate & $A_{n r}=1 \times 10^{8} \mathrm{~S}^{-1}$ \\
Radiative recombination coefficient & $B_{o}=1 \times 10^{-16} \mathrm{~m}^{3} / \mathrm{S}$ \\
Auger recombination coefficient & $C_{a}=3 \times 10^{-41} \mathrm{~m}^{6} / \mathrm{S}$ \\
Dipole moment & $d_{m}=9 \times 10^{-29} \mathrm{~m} . \mathrm{C}$ \\
Dipole relaxation time & $\tau_{i n}=0.1 \times 10^{-12} \mathrm{~S}$ \\
Electrons intraband relaxation time & $\tau_{c}=0.3 \times 10^{-12} \mathrm{~S}$ \\
Holes intraband relaxation time & $\tau_{v}=0.07 \times 10^{-12} \mathrm{~S}$ \\
Spontaneous emission factor & $\beta_{s p}=10^{-4}$ \\
\hline \hline
\end{tabular}

Table 2.2: Extracted Parameters

\begin{tabular}{|c|c|c|c|c|c|c|}
\hline$\alpha_{m}\left[\mathrm{~cm}^{-1}\right]$ & $\alpha_{\text {int }}\left[\mathrm{cm}^{-1}\right]$ & $\mathbb{C}_{\text {tho }}\left[\mathrm{m}^{-3}\right]$ & $I_{\text {tho }}[\mathrm{mA}]$ & $\tau_{\epsilon}[\mathrm{ns}]$ & $\tau_{p h}[\mathrm{ps}]$ & $G_{\text {tho }}\left[\mathrm{s}^{-1}\right]$ \\
\hline 45.006 & 40.032 & $2.134 \times 10^{24}$ & 15.363 & 2.222 & 1.568 & $6.378 \times 10^{11}$ \\
\hline
\end{tabular}

$300 \mathrm{~K}$. The simulated steady state values given in Table(2.2) are in agreement with the results presented in [6].

\subsubsection{Light-Current characteristics}

representing the lasing phenomenon and the parameters affecting the optical output power requires establishing relationship between optical output power and diode drive current. The proposed normalized model is able to predict this relation as shown in Figure 2.5. This assists laser diode designers to predict and analyze the effect of certain parameter. For 
example, understanding the condition of population inversion. There is a threshold current value $I_{t h}$ required to build up a threshold gain to start lasing. Below this transition point, the device acts like a light-emitting diode and most of the emitted light is the spontaneous radiation. The effect of the spontaneous coupling factor $\beta_{s p}$ on the optical output power is also shown in Figure 2.5. The sharpness of the threshold current is mainly affected by $\beta_{s p}$ as shown in this figure and the transition from the non-lasing to the lasing state becomes softer with an increase of $\beta_{s p}$. A sharp increase in the optical output power occurs just above the threshold. In the limiting case of $\beta_{s p}=0$ the threshold current is defined by (2.5.13). Also in this figure, the slope of the linear part of the light-current curve $(d P / d I)$ determines the laser diode modulation efficiency which is another important parameter in the laser design.

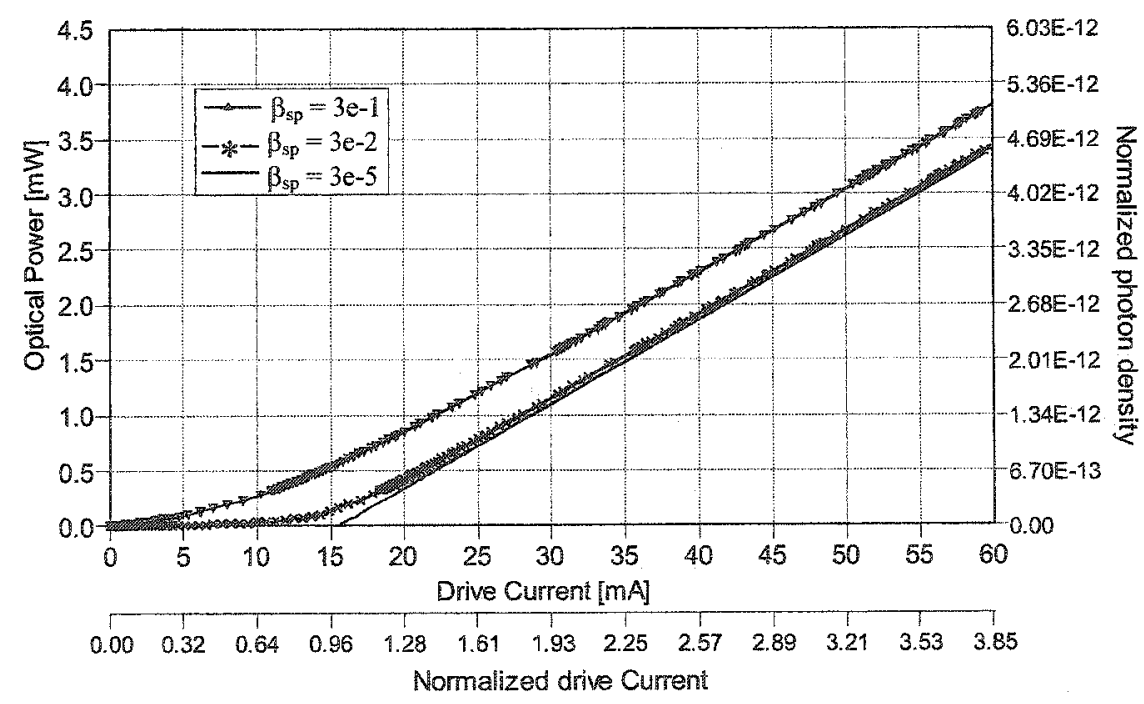

Figure 2.5: Normalized photon density Vs normalized derive current and the corresponding optical output power Vs derive current. As shown, the spontaneous coupling factor $\beta_{s p}$ has a noticeable effect on the light current curve. 


\subsubsection{Variation of carrier population}

Since we assumed an ideal uniform laser in which absorption and leakage do not exist, all the electrons generated by the drive current above the threshold are converted into photons. Thus, above the threshold, the variation of the carrier population with the drive current is nearly constant and does not increase significantly with increasing the drive current as demonstrated by Figure 2.6. Results shown in this figure are in total agreement with the steady state results presented in $[6,46]$

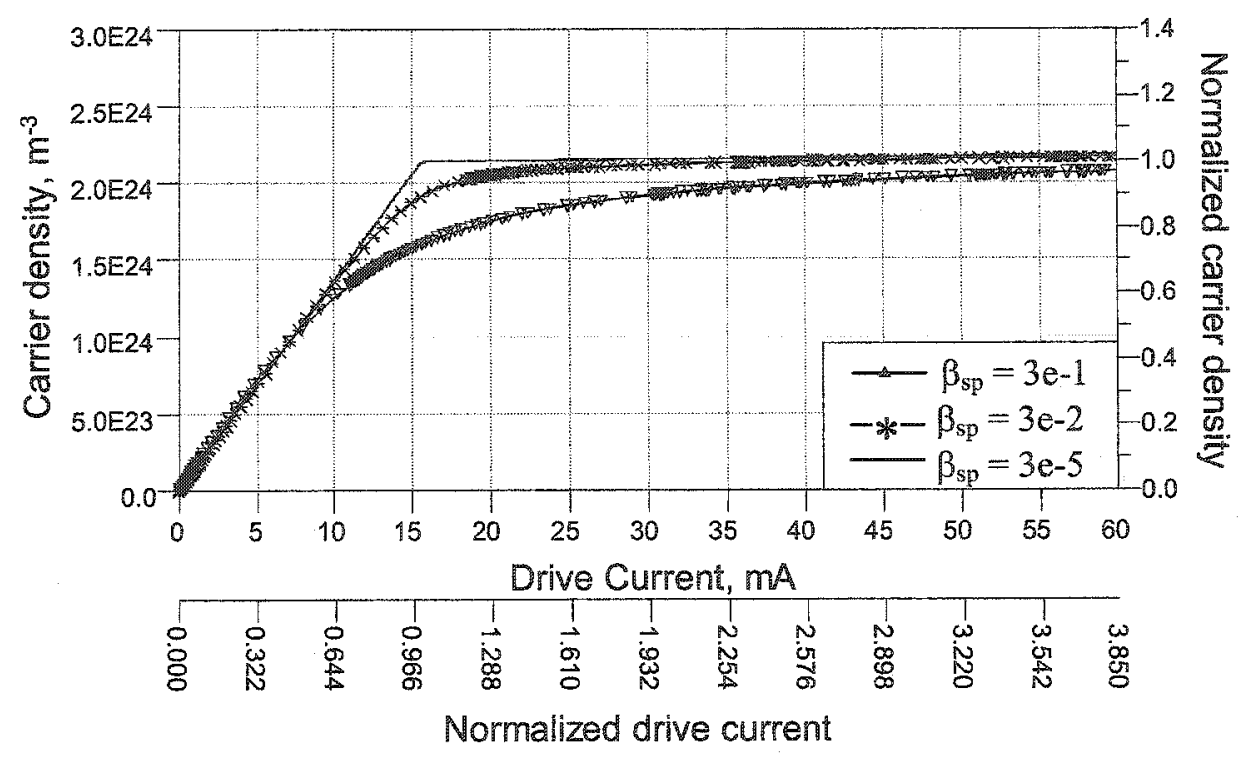

F'igure 2.6: Steady state electron density $\mathbb{C}$ Vs derive current $I(t)$ and the normalized values $\mathbb{C}_{n}$ Vs the normalized drive current.

\subsubsection{Time domain dynamic simulation results}

In section 2.7 .1 we showed how to use the normalized laser model under steady state conditions. In reality, steady state is reached after certain time period called the delay time. This is the time required for the carrier population to reach its threshold value. This delay time is determined by the carrier dynamics alone [6]. The laser diode response in this 
period is called transient response. In optical communications where the modulation frequency is in order of gigahertz, understanding the transient response is important because in this period both electron and photon populations oscillate before reaching steady-state. These oscillations are known as the relaxation oscillations and their frequency is in the gigahertz range. They are used to determine the device response as we will show later. In this section, the normalized model is employed to predict the dynamic characteristics of semiconductor lasers.

Using the parameters shown in Table (2.1), the normalized model predicts the time evolution of the normalized carrier and photon populations under different conditions such as varying $\beta_{s p}$ as shown in Figure 2.7 and Figure 2.8. Figure 2.9 shows the dynamic behav-

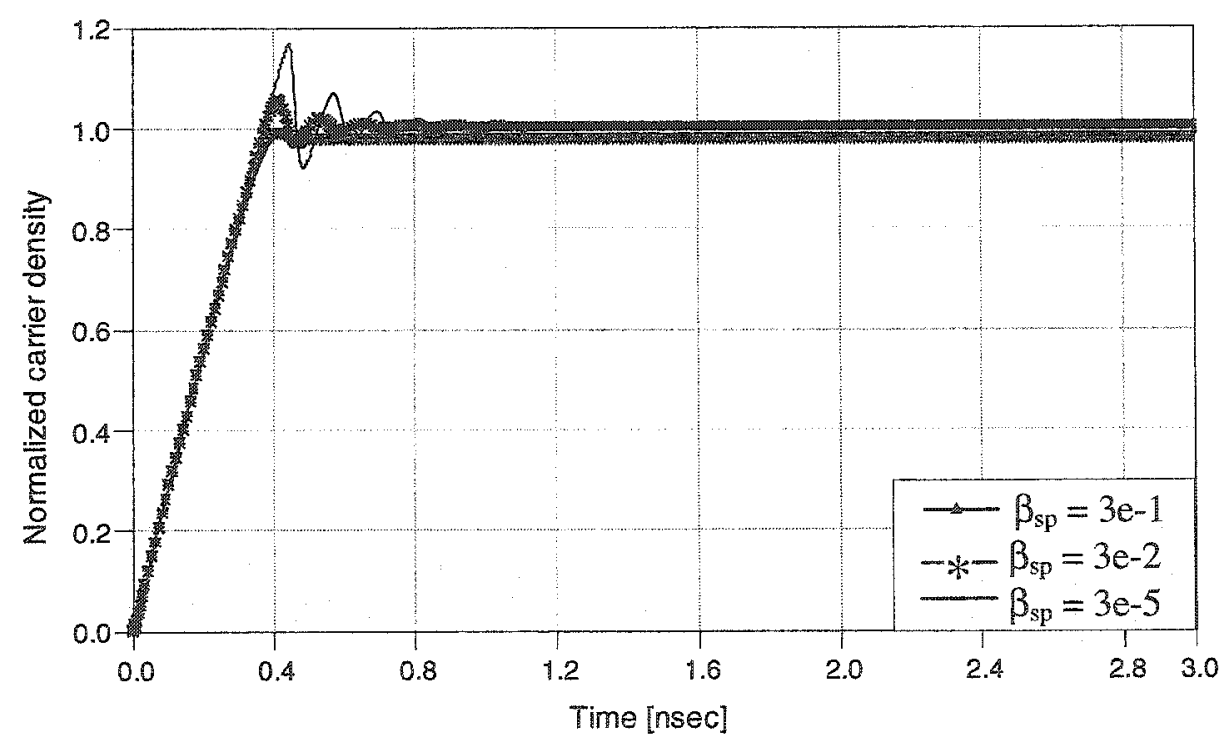

Figure 2.7: Time evolution of the normalized carrier density.

ior of electrons and photons on the same time scale. This figure can be considered as a graphical representation of the relations between the rate equation terms. Also, it is useful in determining the resonance frequency which is the upper limit of the useful bandwidth in the direct modulation. Significant results such as frequency changes and spectral line broadening can also be determined using Figure 2.9 . 


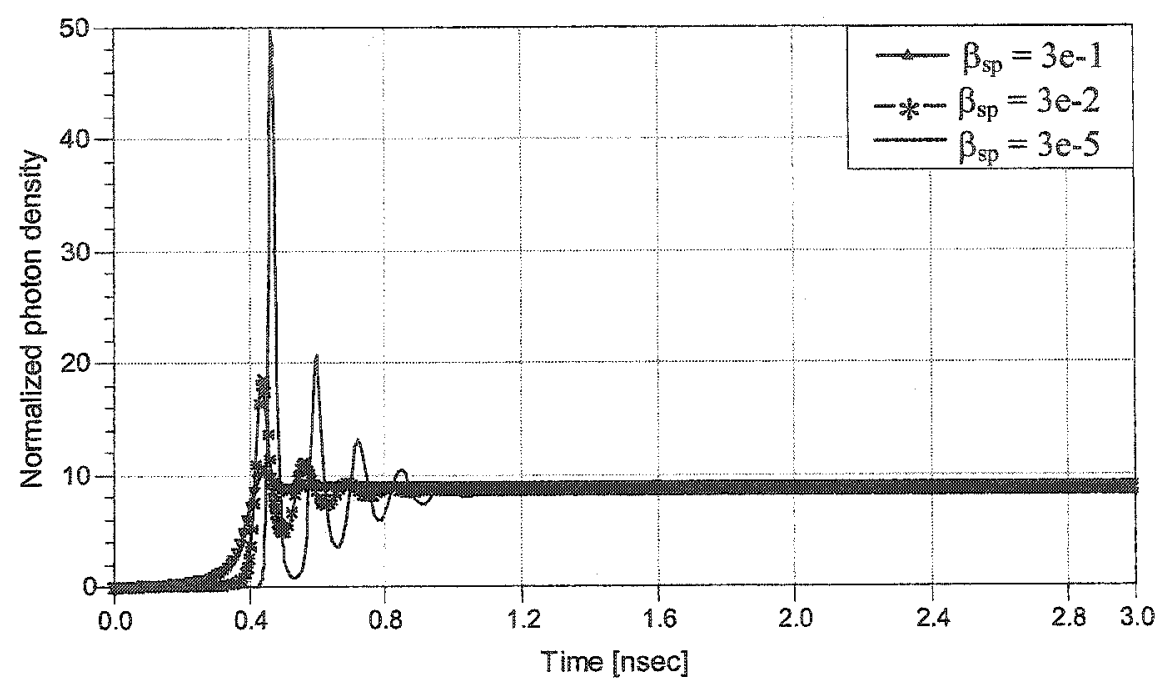

Figure 2.8: Time evolution of the normalized photon density.

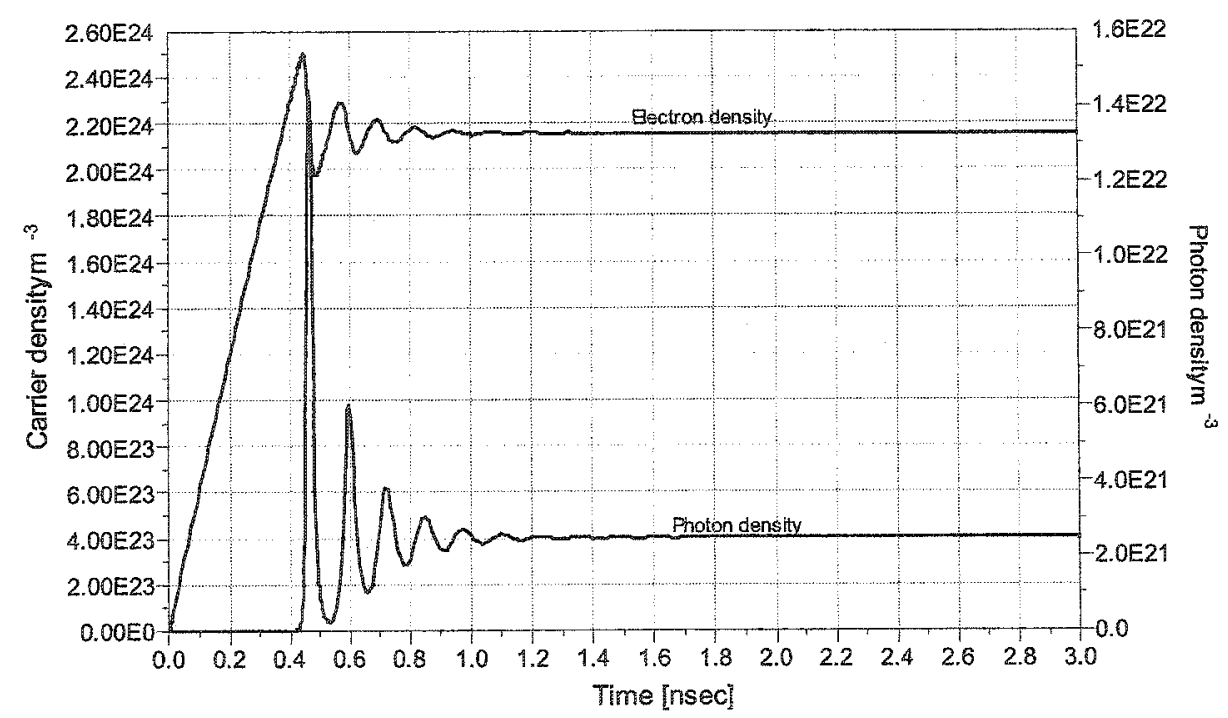

Figure 2.9: Dynamics of the carrier and photon population in time domain showing the turn on spikes and the transient period.

To describe these phenomenon, consider the first spike shown in Figure 2.10 which occurs just after the turn on. Due to increasing the drive current the electron population $\mathbb{C}(t)$ increases and passes up through the threshold value $\mathbb{C}_{t h}$ at time $t 1$, while the photon population in the laser cavity is still essentially zero. Just above $t 1, \mathbb{C}(t)$ surpasses $\mathbb{C}_{t h}$, 


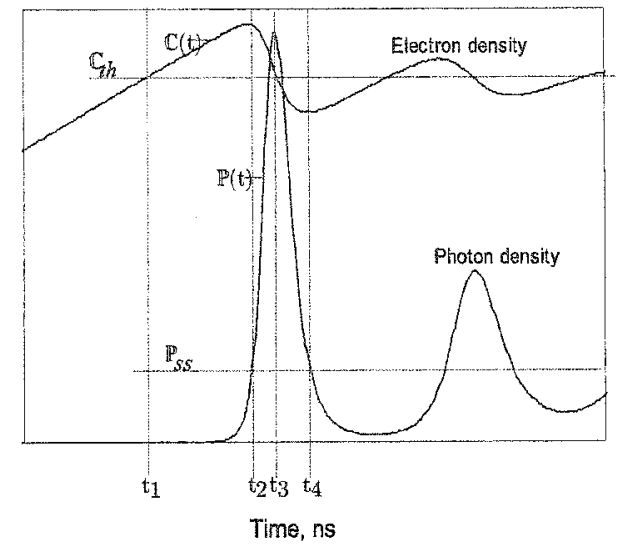

Figure 2.10: First spike from the dynamics of the carrier and photon population shown in Figure 2.9 .

causing the gain to exceeds the loss. Consequently the photon number in the cavity begins to build up exponentially and continues to increase as the pump pushes the carrier population further above the threshold.

Once $\mathbb{P}(t)$ exceeds the steady state level $\mathbb{P}_{s s}$, the intensity of stimulated emission inside the cavity becomes large enough to utilize the excited state atoms at a faster rate than the pump supplies them. Ahead of $t 2, \mathbb{C}(t)$ starts to decrease at a relatively faster rate than its increase, but remains above the threshold level $\mathbb{C}_{t h}$. During this period, the gain remains greater than unity (gain $>$ loss) and $\mathbb{P}(t)$ continues to increase rapidly.

At time $t 3, \mathbb{C}(t)$ approaches $\mathbb{C}_{t h}$ causing the gain to be equal the loss, and $\mathbb{P}(t)$ reaches its peak then begins to fall down but remains large enough to burn up excited state atoms causing $\mathbb{C}(t)$ to continue in its decreasing fashion below $\mathbb{C}_{i h}$. Below $\mathbb{C}_{t h}$, the cavity losses become greater than the laser gain, causing $\mathbb{P}(t)$ to abruptly drop back in value.

At $t 4, \mathbb{P}(t)$ reaches its steady state level and $\mathbb{C}(t)$ reaches a minimum. Immediately after this point, $\mathbb{C}(t)$ begins to build up due to the effect of the injection current, and $\mathbb{P}(t)$ continues to decrease until $\mathbb{C}(t)$ reaches the threshold value again. The same phenomenon is repeated but with decayed amplitudes until $\mathbb{P}(t)$ and $\mathbb{C}(t)$ reach steady state values.

Also from this figure and due to the inverse relation between the electron density and the 
refractive index, the electron density curve can represent the dynamic shift of the central lasing frequency (or chirp or linewidth broadening).

The simulation results presented in this section were obtained while the simulation temperature set to $300 \mathrm{~K}$, and the laser is derived by a current ramp from 0 to $100 \mathrm{~mA}$. The simulated settling time for all cases is 3 nano seconds. The simulated steady state number of carriers was $2.15 \times 10^{8}$ which is in agreement with the results presented in $[6,119]$ and with our steady state analytical solutions. Comparison of carrier and photon density curves in Figure 2.9 shows that the dynamic behavior of the photon number inside the laser cavity is related to the carrier number behavior. This interdependency is exemplified by the time correlation between the peaks and valleys of the waveforms as shown in Figure 2.10 and by the similar settling times for the carrier and photon numbers as described above.

Finally, the time evolution of the optical output power is shown in Figure 2.11. Predictably, the settling time of the output optical power is similar to that of the carrier and photon numbers. Taken together, Figures $2.7-2.9$ and Figure 2.11 indicate that the proposed model can correctly predict the dynamic behavior of semiconductor lasers for low and high laser bias currents.

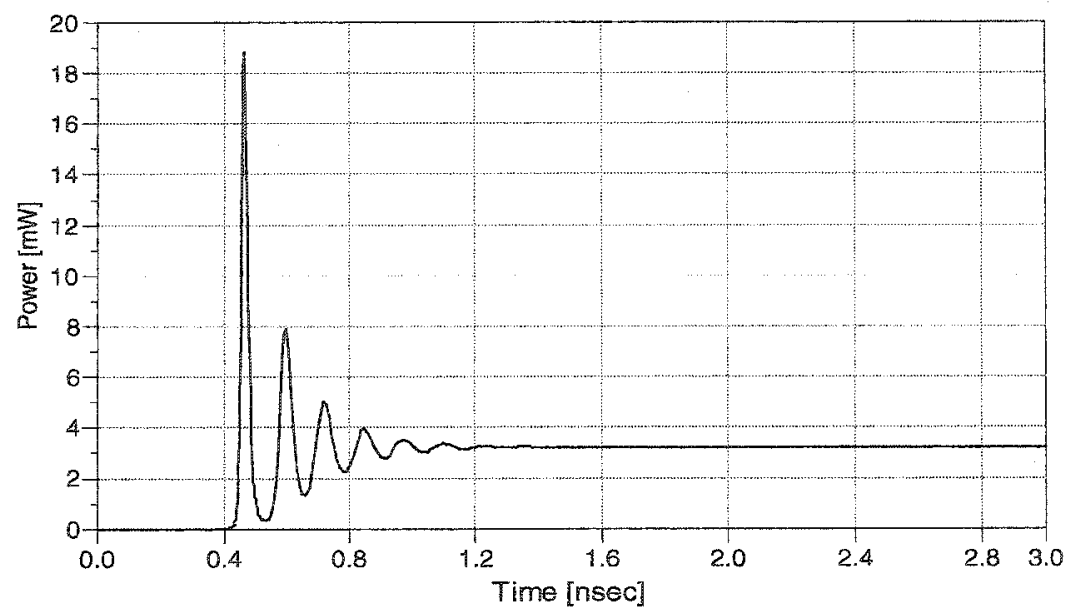

Figure 2.11: Time evolution of the optical output power. 


\subsubsection{Frequency domain simulation results}

Laser modulation response, modulation bandwidth, and relaxation oscillation frequency form the main characteristics of diode lasers intended for optical communication systems. The proposed laser model shown in Figure 2.3 and represented by the diode symbol as shown in Figure 2.12 is used under direct modulation in order to verify that the dynamic behavior of the model is similar to that of a real diode. It is also used to predict the above mentioned characteristics in the frequency domain using the realistic parameters shown in Table (2.1). Both the modified gain formula (MGF) given in (2.5.2) and the conventional gain formula (CGF) given in (2.5.1c) were simulated in order to compare the simulated dynamic behavior using both formulas with the measured dynamic behavior of the real diode [3]. The laser bias was swept from $20 \mathrm{~mA}$ to $100 \mathrm{~mA}$ for a total of ten bias levels. The input signal was a small signal sinusoid, with the input modulation frequency ranging from $0.1 \mathrm{GHz}$ to $15 \mathrm{GHz}$. The simulated laser modulation response is shown in Figure 2.13 for both the modified gain formulation and the conventional gain formulation.

Inspection of the laser modulation response curves, for all bias levels, reveals that the normalized laser modulation response is substantially larger at the resonance frequency of the relaxation oscillations compared to the response at lower frequencies.

Simulation results indicate that a higher relaxation oscillation frequency and a higher normalized modulation peak are predicted using the conventional gain formulation compared to simulation results obtained using the modified gain formulation. Comparison ${ }^{1}$ of the behavior of both sets of modulation responses with published measured results $[9,87,113]$ confirms that the the modified gain formulation behaves in accordance with the measurement results, while the simulated results obtained for conventional gain formulations do not exhibit such performance.

\footnotetext{
${ }^{1}$ The measured results in $[87,113]$ are for different laser parameters.
} 
Published measured results in $[87,113]$ and the measurement results presented later in Chapter 4 indicate that the modulation response peak decreases monotonically with increasing laser bias current. As shown in Figure 2.14, the modulation response peak predicted using a conventional gain formulation does not decrease monotonically with bias current. While, the modulation response peak (in dB) obtained using the modified gain formulation and shown in Figure 2.14 decreases in a linear fashion with bias current when plotted on a logarithmic scale and tends to follow the trend as measured.

The modulation bandwidth and relaxation oscillation frequency are plotted in Figure 2.15. The modulation bandwidth and relaxation oscillation frequency are increasing functions of bias current for both the modified gain formulation and the conventional gain formulation. As shown in Figure 2.15, the relaxation oscillation frequency and modulation bandwidth predicted using the modified gain formulation appear linear when plotted on a linear-logarithmic scale. Generally, the modulation bandwidth and relaxation oscillation frequency are over-estimated when the conventional gain formulation is used compared to results obtained using the modified gain formulation, particularly as the injected current increases, causing an increase in the photon number [3].

In conclusion, the model correctly predicts the variation of the modulation response, modulation bandwidth, and relaxation frequency for a wide range of laser bias currents. It was demonstrated that accurate determination of the laser modulation response at medium to high laser bias currents can be achieved using the proposed gain formulation. 

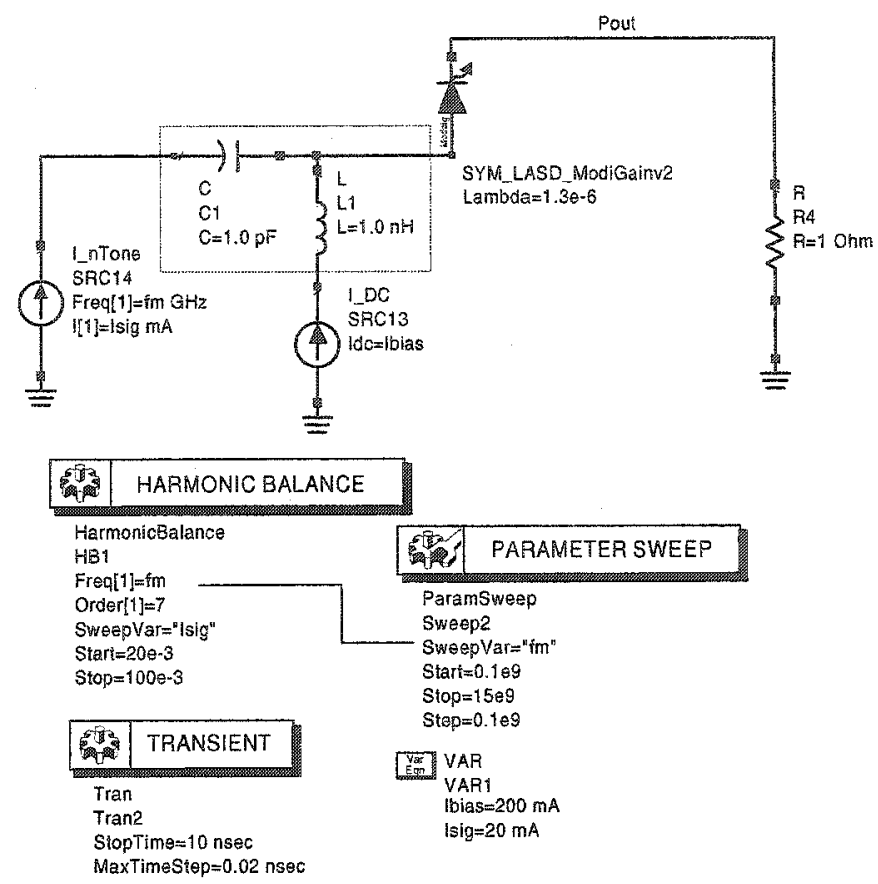

Figure 2.12: Agilent ADS schematic. The diode symbol shown represents the hierarchically defined SDD shown in Figure 2.3 [3].

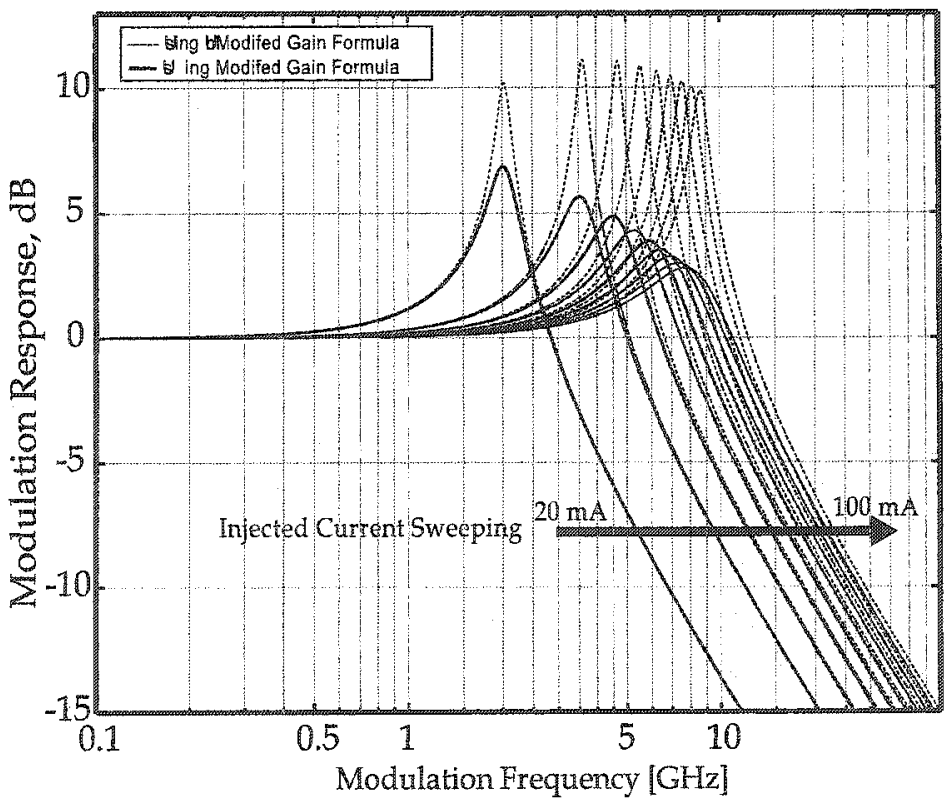

Figure 2.13: Laser modulation response curves. The laser bias current was swept from $20 \mathrm{~mA}$ to $100 \mathrm{~mA}[3]$. 


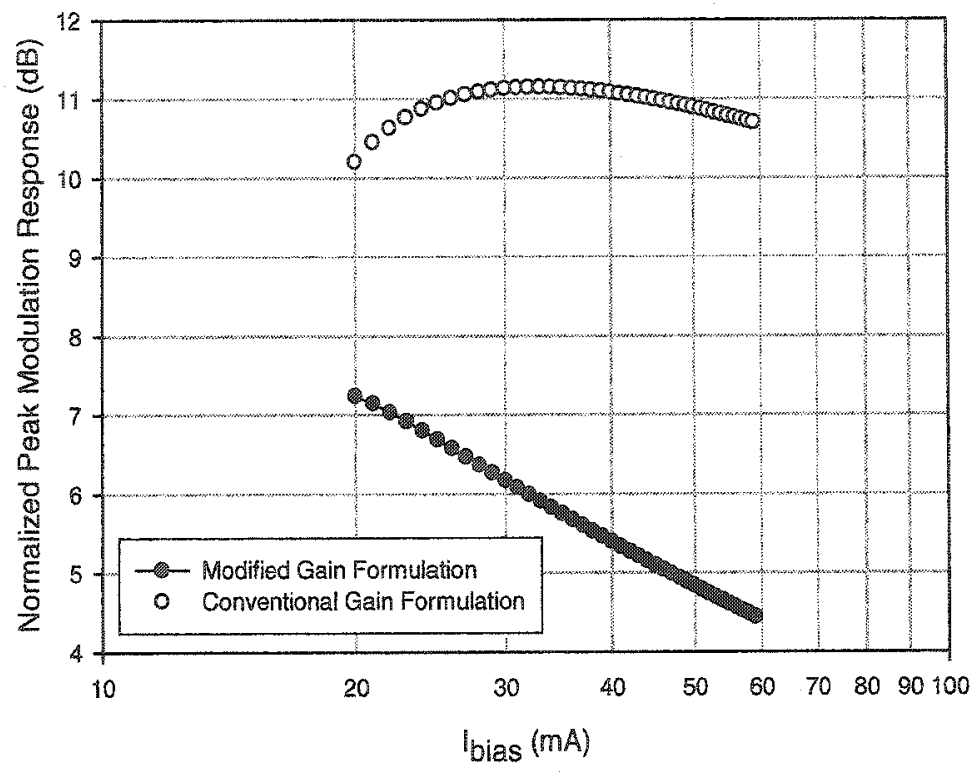

Figure 2.14: Peak modulation response [3].

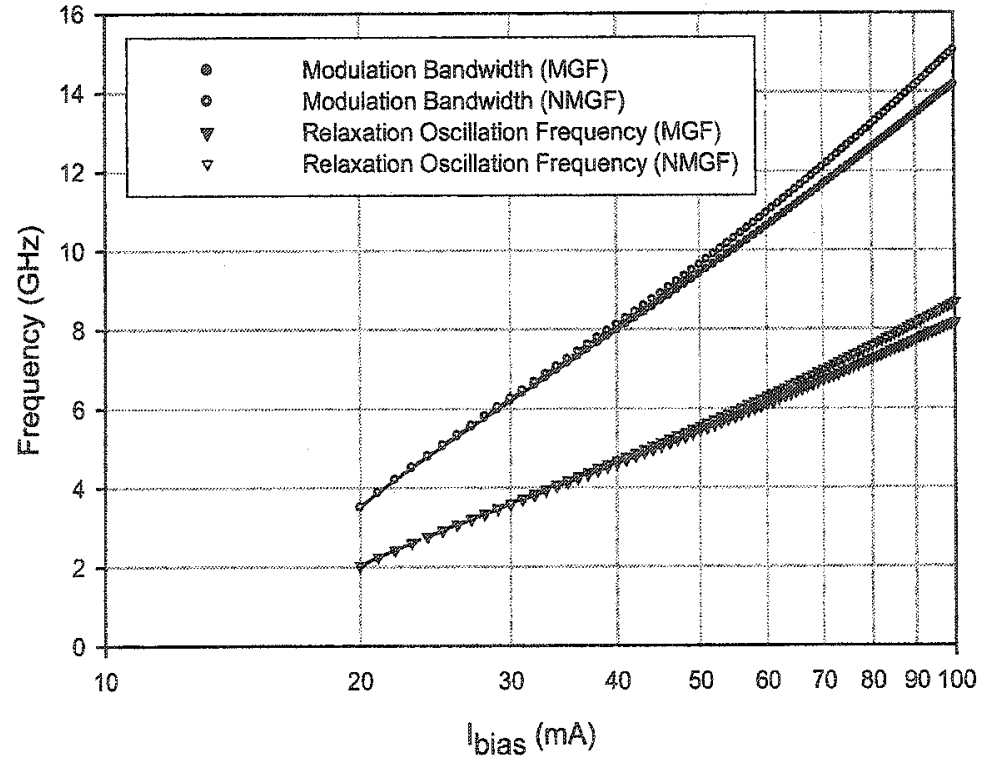

Figure 2.15: Relaxation oscillation frequency and modulation bandwidth derived from Figure 2.13 [3]. 


\subsection{Model advantages and limitations}

Since the proposed model is fundamentally based on the conventional semiconductor laser rate equations, it will inherit all the useful characteristics of these equations such as clarity and simplicity. The conventional rate equations were extended in the proposed model to include the modified gain formulation given by (2.5.2) and to overcome the power limitation. Another advantage of this model is the normalization of the modified rate equations. The proposed normalization technique enhances the convergence of the numerical solvers and makes them suitable for transient, small signal, and large signal analysis.

Implementing these models in Agilent-ADS make them suitable for simulations from the circuit level up to the system level. Hence, the enhanced model is suitable for both small signal and large signal simulations in the time and frequency domains.

The operation of the proposed model is thermally limited to its operation at room temperature. Also, these models can not account for any temperature fluctuations and hence they are not suitable for predicting important analogue optical communication characteristics such as, intensity and phase noise. Dynamic range and distortion enhancements are the main motivation for modifying these models.

To overcome the above mentioned limitations of this model and extend its range of validity, thermal effects, leakage, and noise effects should be factored in the modified model. Including these effects should be simultaneous and with no effect on the suitability of the model for both small and large signal simulations in the time and frequency domains as will be presented in the next chapter. 


\section{Chapter 3}

\section{Modified Semiconductor Laser Models}

Dynamic range, modulation bandwidth, and signal distortion are the main parameters used to analyze, evaluate and enhance the performance of analog optical links $[9,10]$. It is not surprising to find that a gap still exists between experimental reality and optimistic theoretical estimations of these parameters $[19,95,100,114,120-125]$. Thus, it is highly desirable to study how to accurately determine these parameters. Significant physical processes affecting these parameters include gain nonlinearities due spectral hole burning, carrier heating, carrier transport, and gain saturation $[50,53,59,66,82-84,100,114,122-124$, 126-130], Auger recombination [6,93], and 3-D thermal effects [64] have been studied separately. Furthermore, significant models including thermal effects such as those presented in $[32,33,64,65,86,90,101,103-105,109,131]$ have been introduced. However, while they can accurately predict the performance of semiconductor diode lasers including thermal effects they are ill-suited for system level simulations. Existing models are problematic because either they require the solution of large sets of simultaneous equations, or, in order to satisfy the desire for fast simulations, they employ vastly simplified models. Over simplified models are a concern because the various phenomena that define the laser's behavior are complex and closely coupled. To accurately predict laser behavior, a considerable number of physical processes should be considered simultaneously in a self-consistent model. The addition of thermal, leakage and noise effects to the proposed model given in the pre- 
vious chapters renders these models suitable for system level simulations and yet retains the model accuracy in predicting the laser's DC and transient responses. The modified models should be compact, clear, accurate and numerically efficient.

In this chapter, the previously presented model will be modified by adding the thermal effects, leakage current, and noise fluctuations simultaneously.

\subsection{Semiconductor laser models incorporating ther- mal effects}

Pure thermal behavior can be modeled if the cause and location of both the heat generation and dissipation sources of a semiconductor structure are known, or can be precisely determined. However, these sources are purely stochastic in nature and due to many factors. Since the laser rate equations used in this thesis as the basis for modeling the dynamic behavior of semiconductor lasers. We study these rate equations to specify the heat generating mechanisms, in order to augment the model.

The laser diode gain spectrum broadens and its peak location shifts to a longer wave length in response to the increases in the active region temperature. The majority of effects, such as gain broadening and power drop, during static or continuous wave operation are due to the temperature-dependent laser gain and carrier leakage out of the active region $[6,121]$.

\subsubsection{Rate equations temperature dependance}

Investigation of semiconductor laser operation through the rate equations as shown in the previous chapter demonstrates that:

- There is no lasing before threshold. However, spontaneous phenomena is dominant and generated isotropically within the active region. This means that most of this radiation is converted into heat through absorbtion of this radiation by the cladding 
areas surrounding the active region. Losses are then greater than gain in this region of operation.

- After threshold, non-radiative recombination heating (which is the result of absorbing a quantity of energy nearly equal to $\mathbb{E}_{g}$ of each recombination occurrence) is dominant. This explains the relation between both transparency and injected carrier densities and temperature. Also during this period, a portion of the radiated energy is transformed into phonons (due to scattering) generating an extra source of heat in the active region.

We can conclude from the above discussion that the temperature dependence of the rate equations mainly arises from the temperature dependence of the injected and transparency carrier densities.

\subsubsection{Transparent carrier density temperature dependance}

Applying the Bernard-Duraffourg condition [15] and assuming parabolic bands for simplicity of calculations, and assuming the electron ensemble has a quasi-Fermi distribution under the charge neutrality condition with a temperature $T$ equal to the lattice temperature, then as a result of any excitation to the active region in this situation, the carrier ensemble redistributes to form a quasi-Fermi distribution with a temperature different from the lattice temperature. The transparency carrier density is derived in Appendix B and given as:

$$
\mathbb{C}_{t r}=2.5 \times 10^{19}\left(m_{c}^{*} / m_{o}\right)^{3 / 2}(T / 300)^{3 / 2} \exp \left(-\frac{E_{g}}{2 K_{B} T}+\frac{3}{4} \ln \left(\frac{m_{v}^{*}}{m_{c}^{*}}\right)\right)
$$

in which the energy gap $E_{g}$ is in $\mathrm{eV}$ and according to appendix $\mathrm{B}$ it can be represented as a function of temperature as in (3.1.2).

$$
E_{g}(T)=E_{g}(0)-4.3 \times 10^{-4} \frac{T^{2}}{T+T_{o}}
$$


In (3.1.2), $E_{g}$ is in $\mathrm{eV}, \mathrm{T}$ is the average active region temperature given in degrees Kelvin, $T_{0}$ is the characteristic temperature of the active region, and $m_{0}$ is the electron rest mass. Relations for $\left(m_{c}^{*} / m_{o}\right)$ and $\left(m_{v}^{*} / m_{o}\right)$ are given in Appendix $B$; they are related to the material characteristics. Using (3.1.2) and simplifying the expression $T^{2} /\left(T+T_{0}\right)$ by applying Taylor's expansion (3.1.1) leads to the final expression of the transparency carrier density as given by (3.1.3) in $\mathrm{cm}^{-3}$ (see Appendix B for more details).

$$
\mathbb{C}_{t r}(T) \approx \mathbb{C}_{\text {tro }} e^{\frac{T-T_{r}}{T_{o}}}
$$

Where $T_{r}$ is the equilibrium temperature without injection, $\mathbb{C}_{t r o}$ is given by $(\mathbf{B} .0 .13)$ and approximately equal to $1.093 \times 10^{18}\left[\mathrm{~cm}^{-3}\right]$ at $T=300 \mathrm{~K}$ for $\operatorname{In}_{1-x} \mathrm{Ga}_{x} \mathrm{As}_{y} \mathrm{P}_{1-y}$ while the mole fractions $x=0.28$ and $y=0.6$, and lattice matched to InP. Equation (3.1.3) implies that the transparency carrier density is an exponential function of temperature. This is in agreement with the empirical formulations in the literature $[48,121,132]$. An interesting result based on this derivation is that increasing the temperature does not absolutely increase the carrier density. Based on the analytical derivation of the temperature dependance of the carrier density, increasing the temperature will cause more leakage and more reduction in the carrier mobility, resulting in optical output power drop.

\subsubsection{Laser threshold temperature dependance}

Using (3.1.3) in (2.5.9) and (2.5.10) yields that photon life time and threshold current are strong functions of temperature. From (2.5.9) the photon life time is given as,

$$
\tau_{p h}(T)=\frac{1}{\Gamma v_{g}(T) \wp(T)\left(\mathbb{C}_{t h}(T)-\mathbb{C}_{t r}(T)\right)}
$$

From (2.5.10) the threshold carrier density is given as,

$$
\mathbb{C}_{t h}(T)=\frac{1}{\tau_{p h}(T)\left(\Gamma v_{g}(T) \wp(T)\right)}+\mathbb{C}_{t r}(T)
$$


Following the same methodology, the temperature dependance of the differential gain coefficient $\wp$ is given by,

$$
\wp(T)=\wp_{0} e^{\frac{T-T_{g}}{T_{0}}}
$$

in which $\wp_{0}$ is the equilibrium differential gain coefficient, and $T_{g}$ is the characteristic temperature for the differential gain coefficient. For simplicity, we assume that $T_{r}=T_{g}$ for bulk active regions. Substituting (3.1.6) and (3.1.3) in (3.1.5) and simplifying the result leads to the threshold carrier density given by,

$$
\mathbb{C}_{t h}(T) \approx \mathbb{C}_{t h o} e^{\frac{T-T_{r}}{T_{o}}}
$$

in which,

$$
\mathbb{C}_{t h o}=\mathbb{C}_{t r o}+\frac{\left(\alpha_{m}+\alpha_{\text {int }}\right)}{\Gamma \wp_{o}}
$$

Equation (3.1.7) shows that the threshold carrier density is also an exponential increasing function of temperature.

From (3.1.7) and (2.5.13), the temperature dependance threshold current is given as,

$$
I_{t h}(T)=\frac{q V_{c}}{\tau_{e}(T)} \mathbb{C}_{t h o} e^{\frac{T-T_{r}}{T_{o}}}
$$

\subsubsection{Gain formulation including thermal effects}

Gain temperature dependance arises mainly from the temperature dependance of the transparency carrier density and the differential gain coefficient. Indirect temperature dependent parameters, such as index of refraction and group index of refraction (as shown in Appendix ()), will contribute to the temperature dependance of the gain. Substitution of (3.1.3), (3.1.6) and relations for $\bar{n}$ and $n_{g}(T)$ as given in Appendix $\mathrm{C}$ in (2.5.2) leads to the modified thermally dependent gain formulation,

$$
G(\mathbb{C}, \mathbb{P}, T)=\frac{\Gamma \frac{c}{n_{g}(T)} \wp_{o} e^{\frac{T-T_{r}}{T_{o}}}\left(\mathbb{C}(T)-\mathbb{C}_{\text {tro }} e^{\frac{T-T_{r}}{T_{o}}}\right)}{\sqrt{1+\frac{\Gamma \omega_{0}}{\varepsilon_{0} \bar{n} n_{g}(T) I_{s}} \mathbb{P}(T)}}
$$




\subsubsection{Temperature dependance of the carrier recombination time}

The expression of carrier recombination decay rate given in (2.5.11) is a combination of radiative and non-radiative components. The first term, $B \mathbb{C}$, represents the radiative term and it is caused by spontaneous emission, in which $B\left(\mathrm{~cm}^{3} / \mathrm{s}\right)$ is the radiative recombination coefficient (bimolecular recombination coefficient $[6,132]$ ). According to [133], $B$ can be represented as a function of temperature and given as,

$$
B(T)=B_{0} e^{\frac{-E 1}{\bar{K}^{T}}}
$$

in which $B_{o}$ is the radiative recombination coefficient at $300 \mathrm{~K}$ and $E 1$ is the activation energy of the radiative recombination. According to [133], $C_{a}$ can be represented as a function of temperature and given as,

$$
C_{a}(T)=C_{a o} e^{\frac{-E 2}{K_{B} T}}
$$

in which $C_{a o}$ is the Auger recombination coefficient at $300 \mathrm{~K}$ and $\mathrm{E} 2$ is its activation energy. This discussion leads to the relation of the temperature dependance carrier life time given as,

$$
\tau_{e}(T)=\left(B_{o} e^{\frac{-E 1}{K_{B} T}} \mathbb{C}(T)+A_{n r}+C_{a o} e^{\frac{-E 2}{K_{B} T}} \mathbb{C}^{2}(T)\right)^{-1}
$$

in which $A_{n r}$ represents the non-radiative recombination due to trap and surface defects. The last term represents the non-radiative recombination due to Auger recombination.

\subsubsection{Normalized modifed rate equations including thermal effects}

Using the modified gain formula given in (3.1.10) and the temperature dependance parameters such as Auger and spontaneous radiative recombination, threshold carrier density, threshold current and refractive index the modified set of the rate of change of photons 
and carriers including thermal effects are,

$$
\begin{aligned}
\frac{d \mathbb{P}(T, t)}{d t}= & \frac{\Gamma \frac{c}{n_{g}(T)} \wp_{o} e^{\frac{T-T_{r}}{T_{o}}}\left(\mathbb{C}(T, t)-\mathbb{C}_{\text {tro }} e^{\frac{T-T_{r}}{T_{o}}}\right)}{\sqrt{1+\mathbb{P}(T, t) / \mathbb{P}(T)}} \mathbb{P}(T, t)-\frac{\mathbb{P}(T, t)}{\tau_{p h}(T)} \\
& +\frac{\Gamma \beta_{s p} \eta_{s p}(T, t)}{\tau_{e}(T)} \mathbb{C}(T, t) \\
\frac{d \mathbb{C}(T, t)}{d t}= & \frac{I(t)}{q V_{c}}-\frac{\frac{c}{n_{g}(T)} \wp_{o} e^{\frac{T-T_{r}}{T_{o}}}\left(\mathbb{C}(T, t)-\mathbb{C}_{\text {tro }} e^{\frac{T-T_{r}}{T_{o}}}\right)}{\sqrt{1+\mathbb{P}(T, t) / \mathbb{P}_{s}(T)}} \mathbb{P}(T, t)-\frac{\mathbb{C}(T, t)}{\tau_{e}(T)}
\end{aligned}
$$

in which $\mathbb{P}_{s}(T)$ is a function of temperature through the index and group index of refractions. The internal quantum efficiency in (3.1.14) is a function of temperature as given in (3.1.16) [6]. $\eta_{s p}(T, t)$ is 1 for ideal active media.

$$
\eta_{s p}(T, t)=B_{o} e^{\frac{-E 1}{K_{B} T}} \mathbb{C}(T, t) \tau_{e}(T)
$$

Applying the normalization methodology presented in Section 2.6.2 to (3.1.14) and (3.1.15) gives the normalized rate of change of photons and carriers as,

$$
\begin{aligned}
\frac{d \mathbb{P}_{n}(T, t)}{d t}= & \frac{\left(\mathbb{C}_{n}(T, t)-\mathbb{C}_{t r}^{n}(T)\right)}{\tau_{p h}(T)\left(1-\mathbb{C}_{t r}^{n}(T)\right)} \frac{\mathbb{P}_{n}(T, t)}{\sqrt{1+\mathbb{P}_{r}^{n}(T, t)}} \\
& -\frac{\mathbb{P}_{n}(T, t)}{\tau_{p h}(T)}+\left(\beta_{s p} B_{o} \tau_{e}(T) \mathbb{C}_{t h o} e^{\left(\frac{T-T_{r}}{T_{o}}-\frac{E_{1} q}{K_{B} T}\right)}\right) \mathbb{C}_{n}^{2}(T, t) \\
\frac{d \mathbb{C}_{n}(T, t)}{d t}= & \frac{I(t)}{q V_{c} \mathbb{C}_{t h}(T)}-\frac{\left(\mathbb{C}_{n}(T, t)-\mathbb{C}_{t r}^{n}(T)\right)}{\tau_{e}(T) \tau_{p h}(T)\left(1-\mathbb{C}_{t r}^{n}(T)\right)} \frac{\mathbb{P}_{n}(T, t)}{\sqrt{1+\mathbb{P}_{r}^{n}(T, t)}} \\
& -\frac{\mathbb{C}_{n}(T, t)}{\tau_{e}(T)}
\end{aligned}
$$

\subsubsection{Model implementation using symbolically defined devices}

The semiconductor laser models defined by equations (3.1.17) and (3.1.18) were implemented using Symbolically Defined Devices (SDDs) in Agilent-ADS. The three-port SDD laser model presented in Section 2.6 .3 was enhanced by adding the thermal effects. The 
implemented model is suitable for both small and large signal simulations including transient and steady state simulations in the time and frequency domains. Figure 3.1 shows the schematic symbol for the three-port SDD used to implement the normalized thermally modified laser rate equations while the simulation setup used for time domain simulations is illustrated in Figure 3.2.

\subsubsection{Simulation results}

The proposed model shown in Figure 3.1 was used in the simulation setup shown in Figure 3.2 to predict the dynamic characteristics of laser diodes using realistic parameters for InGaAsP-InP semiconductor lasers, such as those shown in Tables 2.1 and 3.1. Figure 3.3

Table 3.1: Parameters for an InGaAsP/InP semiconductor laser diode and other general physical constants

\begin{tabular}{||l|l||}
\hline \hline \multicolumn{1}{|c|}{ Parameter definition } & \multicolumn{1}{|c||}{ Symbol \& Value } \\
\hline \hline Characteristic temperature & $T_{o}=70 \mathrm{~K}$ \\
Equilibrium Temperature without injection & $T_{r}=300 \mathrm{~K}$ \\
Mole fractions (material composition fractions) & $x=0.282 \& y=0.6$ \\
Transparency carrier density & $\mathbb{C}_{t r o}=1 \times 10^{24} \mathrm{~m}^{-3}$ \\
Band gap energy at ambient temperature & $E_{g}(300)=1.003$ \\
Conductivity of the medium & $\sigma=0.372 \times 10^{2} \Omega^{-1} \mathrm{~m}^{-1}$ \\
Radiative coefficient activation energy & $E 1=8.61 \times 10^{-3} \mathrm{eV}$ \\
Auger coefficient activation energy & $E 2=5.16 \times 10^{-2} \mathrm{eV}$ \\
Permeability of free space & $\mu_{o}=1.25664 \times 10^{-6} \mathrm{H} / \mathrm{m}$ \\
Permittivity of free space & $\varepsilon_{o}=8.85419 \times 10^{-12} \mathrm{~F} / \mathrm{m}$ \\
Boltzman's Constant & $K_{B}=1.38 \times 10^{-23} \mathrm{~J} / \mathrm{K}$ \\
Electron charge & $q=1.6 \times 10^{-19} \mathrm{C}$ \\
Speed of light in free space & $c=3 \times 10^{8} \mathrm{~m} / \mathrm{s}$ \\
Planck's Constant & $h=6.62607 \times 10^{-34} \mathrm{Js}$ \\
\hline \hline
\end{tabular}

illustrates the threshold current for increasing temperature. The plotted threshold current is calculated using the proposed model. Temperatures range from $250 \mathrm{~K}$ to $400 \mathrm{~K}$. The range from $250 \mathrm{~K}$ to $350 \mathrm{~K}$ is used in this figure. To further demonstrate the capability of the model, Figure 3.4 illustrates the behavior of the optical gain versus temperature. 

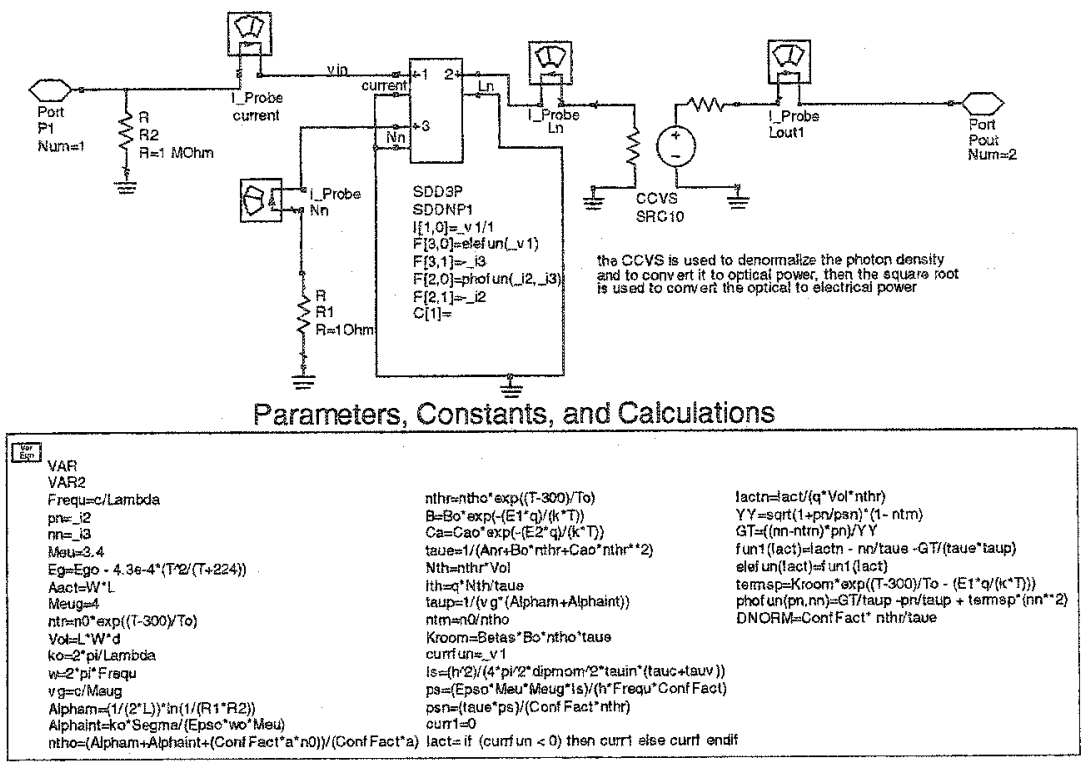

Figure 3.1: The schematic symbol and port equations for a 3-port modified SDD including the temperature effects.

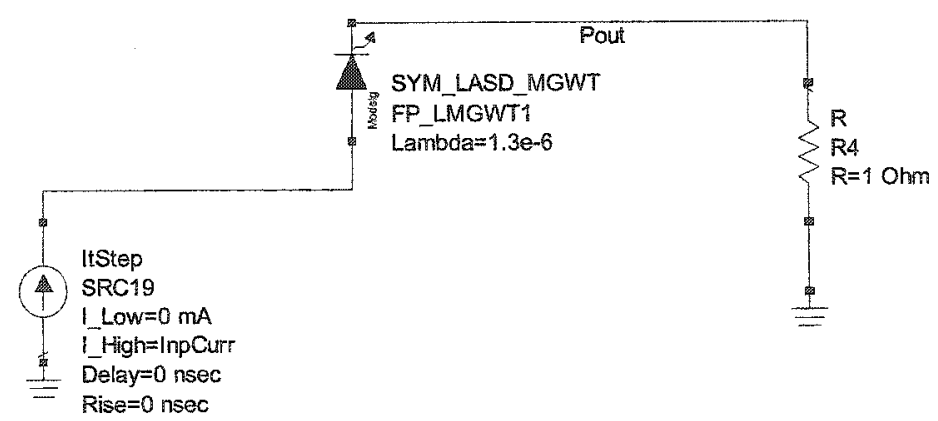

Test Benches and Simulation Parameters

\begin{tabular}{|c|c|c|}
\hline $\begin{array}{l}\text { Var } \\
\text { bor }\end{array}$ & TRANSIENT & PARAMETER SWEEP \\
\hline $\begin{array}{l}\text { VAR1 } \\
\text { InpCurr }=50 e-3 \\
\text { bias }=45 e-3 \\
T=300\end{array}$ & $\begin{array}{l}\text { Tran } \\
\text { Tran3 } \\
\text { StopTine }=15 \text { nsec }\end{array}$ & $\begin{array}{l}\text { ParamSiweep } \\
\text { Sweep5 } \\
\text { SweepVar="T" } \\
\text { SiminstanceName[1] }=\text { "Tran3" } \\
\text { Starn=250 } \\
\text { Stop }=400\end{array}$ \\
\hline
\end{tabular}

Figure 3.2: HP-ADS schematic diagram for the simulation setup. The diode symbol shown represents the hierarchically defined SDD shown in Figure 3.1. The output power is represented by a current in this simulation.

From this figure, the optical gain increases with temperature up to a critical temperature after which it decreases. Decreasing gain corresponds to an increase in the losses which are proportional to the reduction in carrier mobility at elevated temperatures. Reduced 


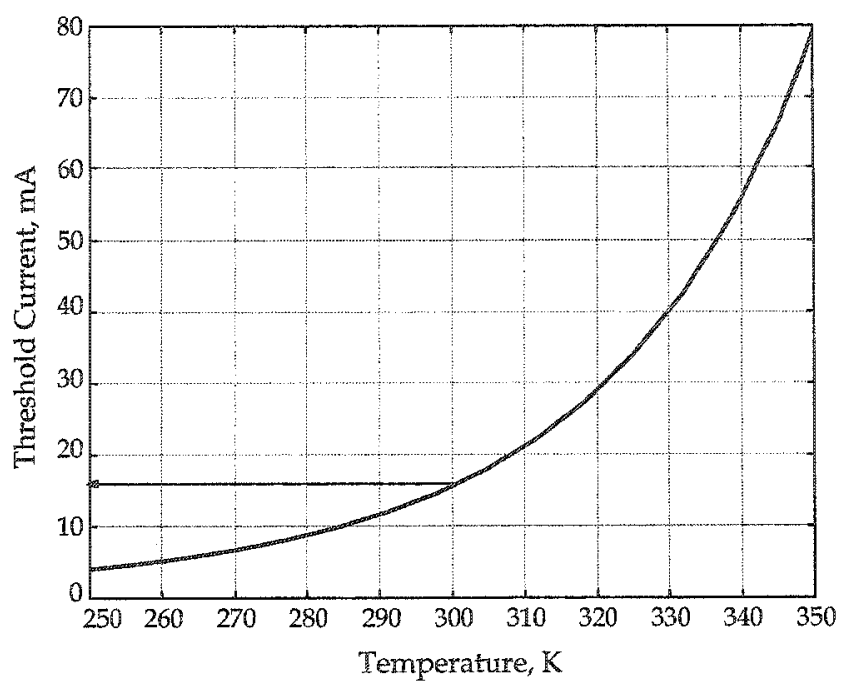

Figure 3.3: Threshold current against temperature as extracted from the model. Temperatures range from $250 \mathrm{~K}$ to $350 \mathrm{~K}$. The arrow is pointing to the threshold current value at room temperature.

gain leads to the output power drop as shown in Figure 3.6. The point at which the gain becomes zero corresponds to zero output power as well. The temperature at which the gain (the output power) vanish is the critical temperature; approximately $340 \mathrm{~K}$ for this laser. Figures 3.5 and 3.6 illustrate the ability of the proposed model to predict the

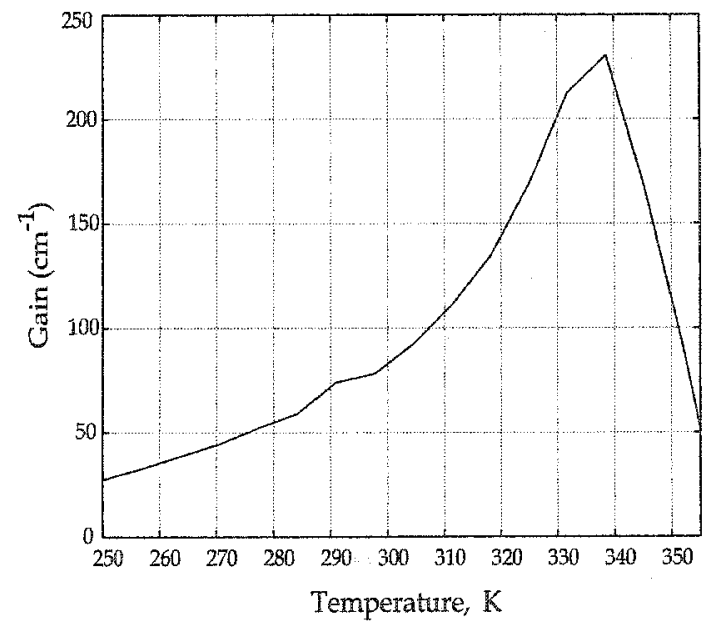

Figure 3.4: Optical gain as a function of temperature. Temperatures range from $250 \mathrm{~K}$ to 360 $\mathrm{K}$. 
influence of the temperature change on the transient response of the semiconductor laser diode. Figure 3.5 illustrates the influence of temperature change on the carrier density while Figure 3.6 illustrates the time evolution of the optical output power and its temperature dependance for a simulated time of $10 \mathrm{~ns}$. A stepped current applied to the laser brings the laser from sub-threshold to above threshold. The simulation was repeated for temperatures from $300 \mathrm{~K}$ to $380 \mathrm{~K}$ using steps of $5 \mathrm{~K}$. As shown in Figures 3.5 and 3.6 the carrier density and output power are both strong functions of temperature. Beyond a critical temperature ( $\mathrm{T}>335 \mathrm{~K}$ as shown in Figure 3.6) the laser output power drops to zero. The simulated steady state number of carriers at room temperature $(300 \mathrm{~K})$ was

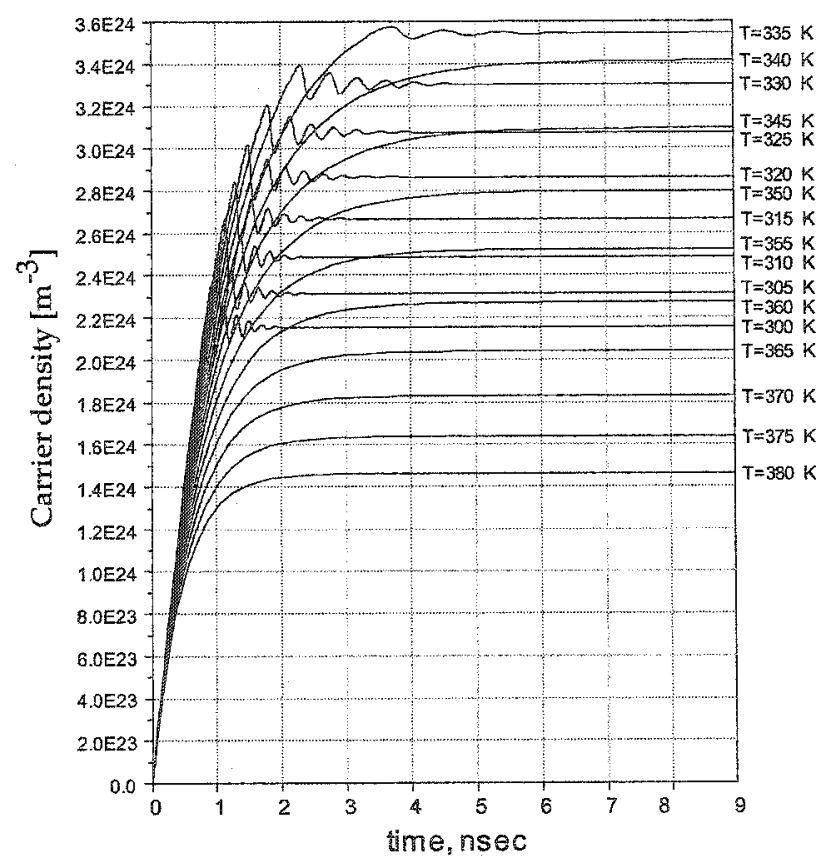

Figure 3.5: Time evolution of carrier density as a function of temperature. Temperature increased from $300 \mathrm{~K}$ to $380 \mathrm{~K}$ using steps of $5 \mathrm{~K}[2]$.

$2.15 \times 10^{8}$ which is in agreement with the results presented in [6]. Comparison of Figure 3.5 and 3.6 shows that the dynamic behavior of the output power is related to the carrier number behavior. This interdependency is exemplified by the time correlation between the 


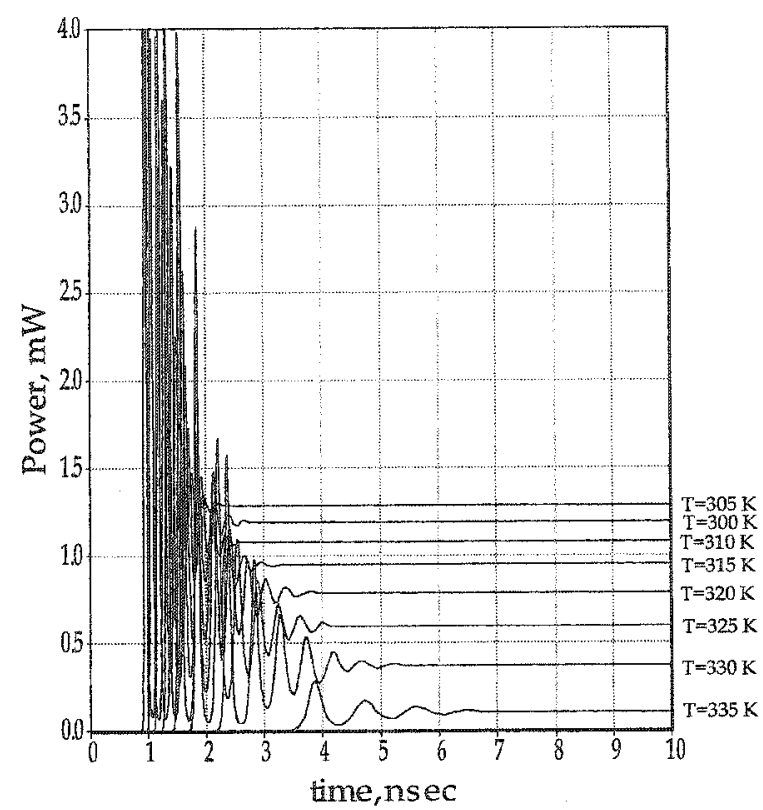

Figure 3.6: Time evolution of the optical power as a function of temperature. Temperature range from $300 \mathrm{~K}$ to $380 \mathrm{~K}$ using steps of $5 \mathrm{~K}[2]$.

peaks and valleys of the plots shown in Figure 3.5 and 3.6 and by the similar settling times for the carrier number and the optical power. Predictably, the settling time of the output optical power is similar to that of the carrier and photon numbers. Figure 3.6 reveals that the peak overshoot and the relaxation oscillation frequency are both decreasing functions of temperature. The time required to reach steady state operation is an increasing function of temperature, implying that the modulation bandwidth is also a decreasing function of temperature as we will show below in Figures 3.7 and 3.8. In the Agilent-ADS simulator, direct modulation of a laser diode with a sinusoidal current signal was used to predict some of the important characteristics extracted from the modulation response of the semiconductor laser. The laser bias was swept from $20 \mathrm{~mA}$ to $100 \mathrm{~mA}$ for a total of ten bias levels. The input signal was a small signal sinusoid with amplitude $1 \mathrm{~mA}$, and the input modulation frequency ranged from $0.1 \mathrm{GHz}$ to $15 \mathrm{GHz}$. Comparison ${ }^{1}$ with published mea-

\footnotetext{
${ }^{1}$ The measured results in [113] are for different laser parameters than in $[6,21]$.
} 
sured results [113], measured results illustrated in Chapter 4, and steady state analytical solution results [6], indicates that the model behaves in accordance with measured results and correctly predicts the steady state values.

Figures 3.7 and 3.8 show the simulated laser modulation bandwidth and relaxation oscillation frequency for different bias currents and different temperatures. Finally, we can

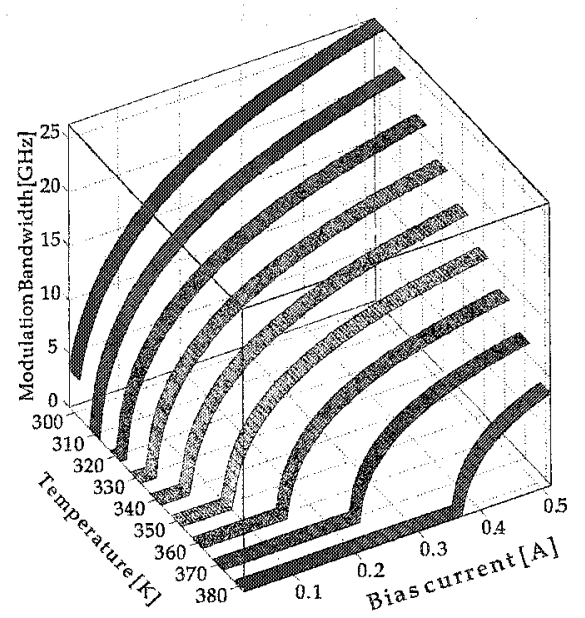

Figure 3.7: Modulation bandwidth for different bias currents and different temperatures [4].

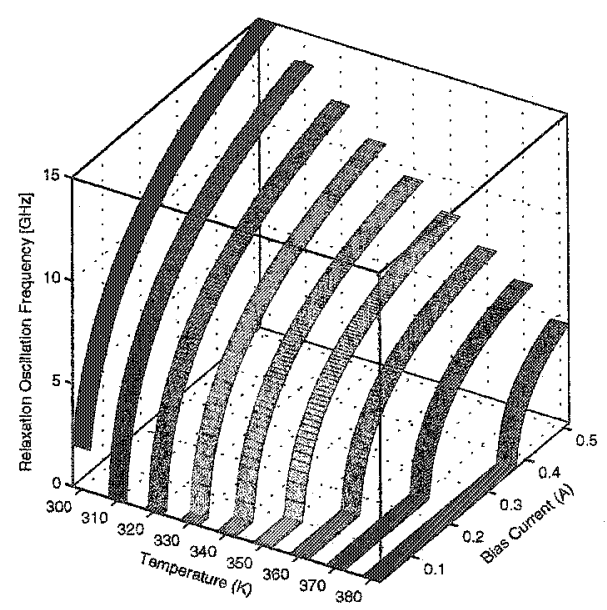

Figure 3.8: Laser relaxation oscillation frequency for different bias currents and different temperatures $[4]$.

conclude that temperature is an important consideration in models intended for simula- 
tions of analog optical links. Taken together, Figure $3.5-$ Figure 3.8 indicate that the proposed model can correctly predict the dynamic behavior of semiconductor lasers for high laser bias currents.

\subsection{Enhanced thermally modified models by includ- ing leakage current effects}

Thermal leakage has a severe impact on device performance [121]. Based on the experimental studies, thermal leakage of carriers out of the active region is a source of laser non-linearity [134-139]. One theory behind the leakage current is that the bandgap of the active layer gets smaller as the device temperature increases as shown in equation (B.0.11) in Appendix B. Furthermore, increasing the device temperature increases the carrier density inside the active layer due the hole transport across the heterostructure. Additionally, hole transport in the forward direction across the active region determines the shape of the barrier confining electrons [134], and increasing the device temperature increases the position of the the active layer's quasi-Fermi levels relative to the bandgap, as shown in Figures 3.9 and 3.10. Consequently, the active layer gets worse at adequately confining carriers. Eventually, a sizeable fraction of injected carriers do not contribute to the recombination process inside the active region. The process depends on several parameters such as the valence band discontinuity and the doping in the depleted region of the cladding layer adjacent to the active layer. Part of the external current does not pass through the active region, causing a reduction in the lasers overall efficiency [121,134-139]. Leakage becomes a dominant infuence on the lasers operation $[121,134,135]$. Leakage currents have been studied and presented based on the thermionic theory of barrier injection [5]. Scott et al. [140] modeled the carrier leakage using an approximate homo-junction-diode relationship proportional to the bandgap of the confinement layers surrounding the active region and the active regions quasi-Fermi-level separation. Their model is based on detailed 


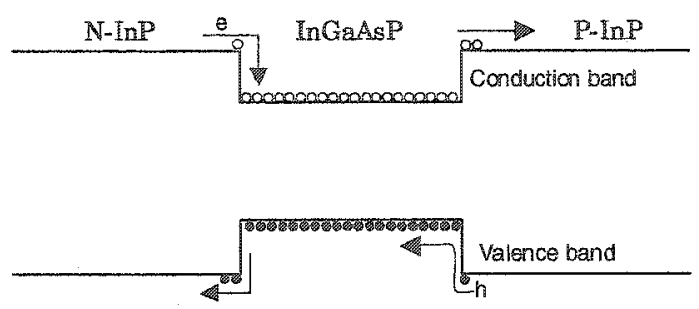

Figure 3.9: Schematic illustration of the hetrojunction leakage current $[5,6]$.

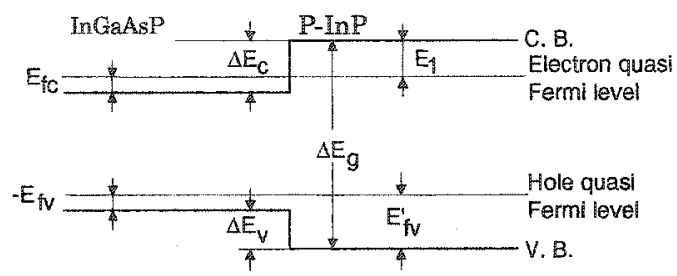

Figure 3.10: Energy band diagram near the boundary of P-InP cladding layer and InGaAsP active layer. $E_{f c}$ and $E_{f v}$ are the electron and hole quasi-Fermi levels, $\Delta E_{c}$ and $\Delta E_{v}$ are the conduction and valence band barrier heights, and $E_{f v}^{\prime}$ and $E_{f v}$ are the hole quasi-Fermi levels in the p-cladding layer and active layer respectively $[5,6]$.

calculations of the leakage current as a function of carrier density, but does not include an analytic model of the thermal dependance of the carriers. Instead Scott et al used curve-fitting to model the carrier and temperature dependence. The distance over which the barrier potential energy in the vicinity of the active layer varies by $K_{B} T$ is typically longer than the mean free path of holes in the depleted region [134]. Therefore, leakage current can be reasonably described by the electron and hole drift and diffusion from the edge of the active layer to the cladding layers.

Diffusion rather than the thermionic theory is used in this thesis, based on this observation. Solving the continuity equation under suitable boundary conditions gives the total leakage current as (for details see Appendix D),

$$
I_{l e a k}=\left(J_{e}+J_{p}\right) A_{a c t}
$$

in which $J_{e}$ is the electron leakage current density and is given as,

$$
J_{e}=q \sqrt{\frac{D_{e}}{\tau_{e}}}\left(\operatorname{coth} \sqrt{\frac{\tau_{e}}{D_{e}}}\right) \mathbb{C}_{b}
$$


and the hole leakage current density can be given as,

$$
J_{p}=-q \sqrt{\frac{D_{p}}{\tau_{p h}}}\left(\operatorname{coth} \sqrt{\frac{\tau_{p h}}{D_{p}}}\right) \mathbb{P}_{b}
$$

Based on calculations of the mobility and diffusion length of the electrons and holes we neglect the effect of the hole leakage in our model. The effect of the leakage current is added to the model by splitting the injection current into two components, one called $I_{e f f}$ which refers to the part of the external current that passes through the active region, and the leakage current which is represented as, $\mathbb{I}_{\text {leak }}$, such that,

$$
I_{e f f}(t)=I(t)-I_{\text {leak }}
$$

Using equation (3.2.4) in (3.1.18), the enhanced laser model is similar to the model given by (3.1.17) and (3.1.18) except the first term in equation (3.1.18) is,

$$
\frac{I_{e f f}(t)}{q V_{c} \mathbb{C}_{t h}}=\frac{I(t)}{q V_{c} \mathbb{C}_{t h}}-\frac{I_{l e a k}(t)}{q V_{c} \mathbb{C}_{t h}}
$$

\subsubsection{Simulation results}

The model shown in Figure 3.1 was enhanced by adding the leakage current term. The simulation setup shown in Figure 3.2 was used to study the effect of the leakage current on the performance of the laser diode. Device temperature and the carrier density were examined. The dynamic characteristics of laser diodes with and without the leakage current was simulated for various temperatures. The InGaAsP-InP semiconductor laser parameters shown in Tables 2.1 and 3.1 were used in the simulations. Figure 3.11 illustrates the variation of the leakage current with the device temperature for different carrier densities. The figure also shows that leakage current is negligible at lower temperatures but increases drastically after a threshold value. This threshold values depends on the type of laser and the laser parameters. For the case shown in Figure 3.12, the critical temperature was 330 K. Figure 3.12 illustrates the behavior of the leakage current in the time domain for several 
temperatures. There is a significant variation in the time domain response of the leakage current after the threshold temperature is exceeded. This increase is due to the relation between the temperature and the carrier density, as discussed below. Figures 3.13 and

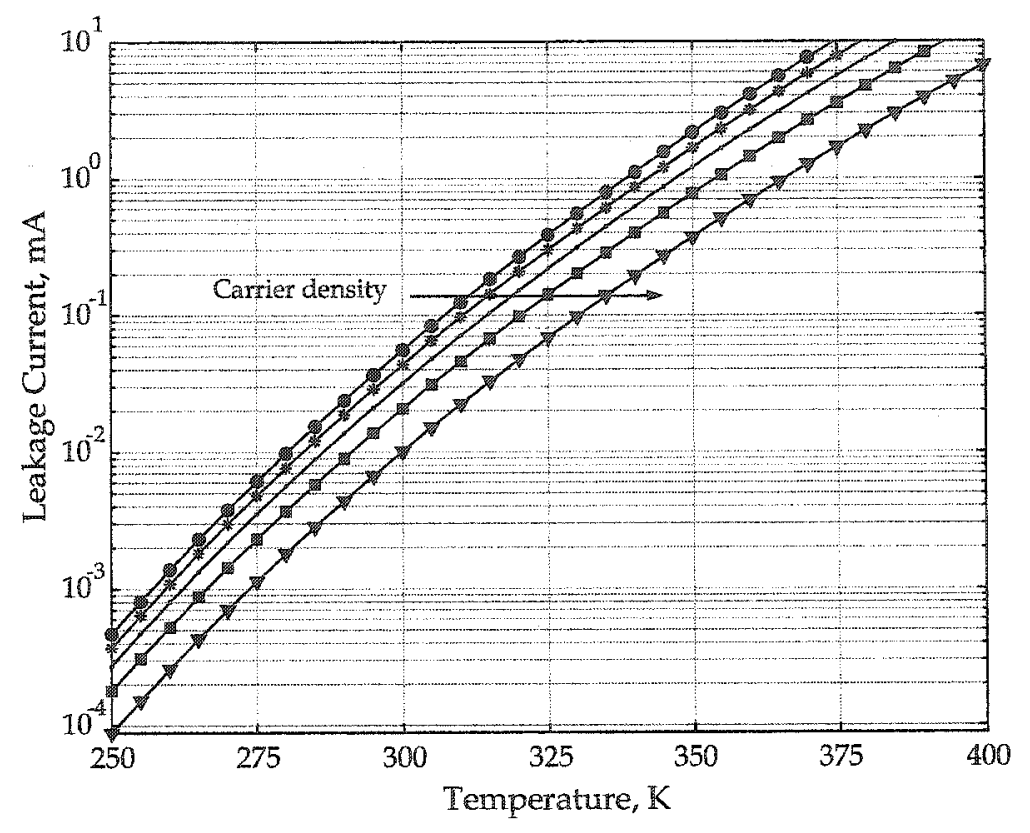

Figure 3.11: Variation of the leakage current with temperature for different carrier densities.

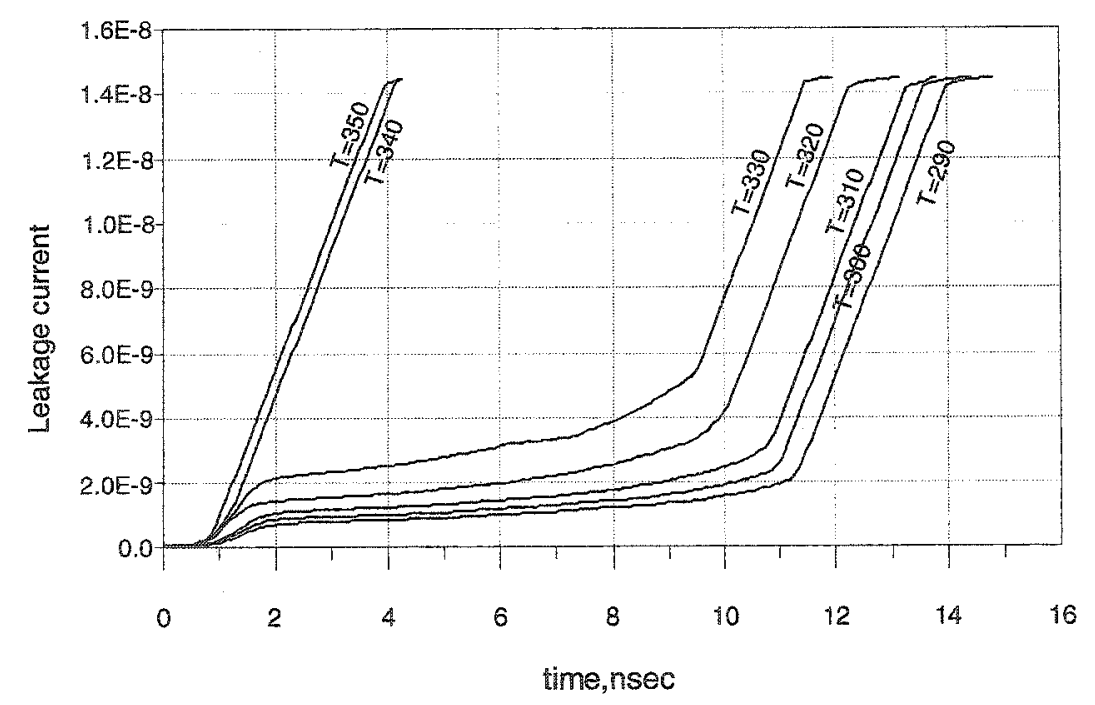

Figure 3.12: Instantaneous leakage current and its variation with temperature. Note the rapid increase of the leakage current when $\mathrm{T}>330 \mathrm{~K}$ 
3.14 illustrate the variation of the leakage current with the carrier density. Figure 3.14 demonstrates this variation at low temperatures. Figure 3.14 shows also that the leakage current is an increasing function of the carrier density and becomes more sensitive to the variation of carrier density when the device temperature gets higher. Eventually, the leakage current saturates with respect to the carrier density, irrespective of the device temperature as shown in Figures 3.14 and 3.12. Figures $3.15-3.17$ illustrate the ability

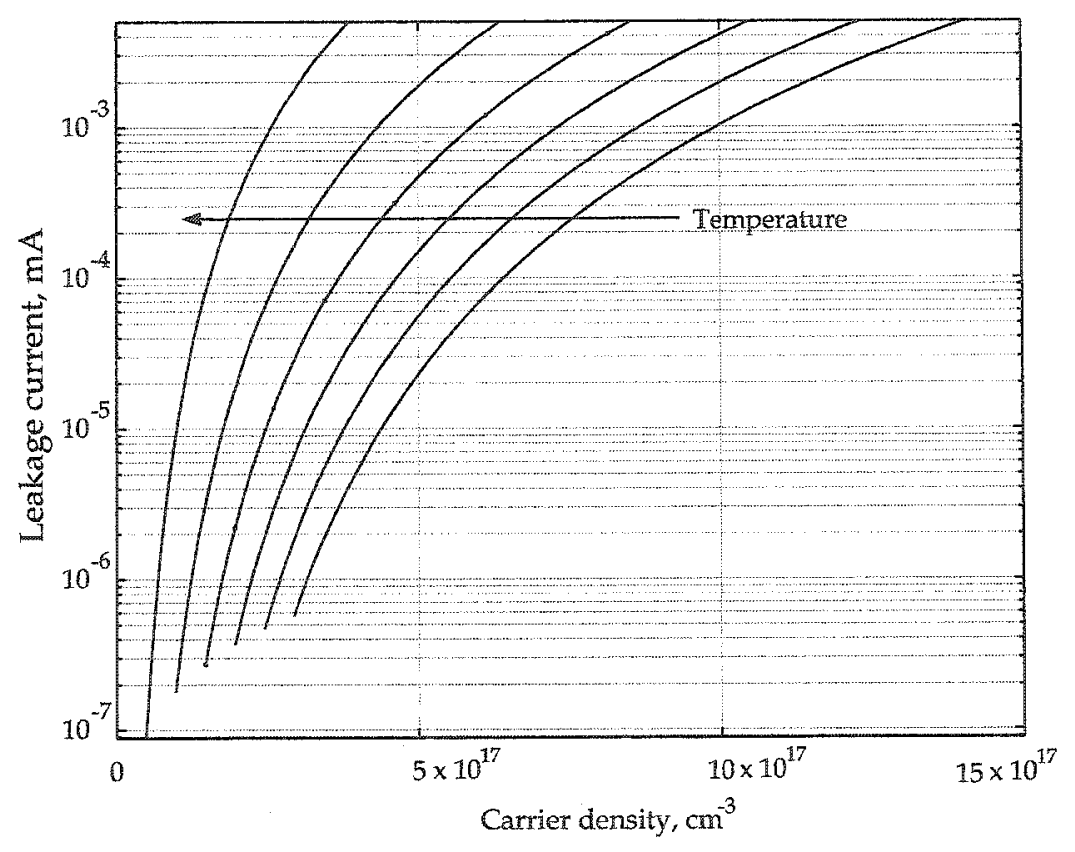

Figure 3.13: Variation of the leakage current with carrier density.

of the model to predict the influence of temperature change on the transient response of a semiconductor laser diode while including the effects of leakage current. A stepped current applied to the laser brings the laser from sub-threshold to above threshold. The simulation was repeated for temperatures from $250 \mathrm{~K}$ to $400 \mathrm{~K}$ using steps of $10 \mathrm{~K}$, with and without the effect of leakage current. As shown, the laser carrier density and output optical power are both strong functions of temperature. Beyond a critical temperature, there is a reduction in carrier mobility and the output power drops to zero. 


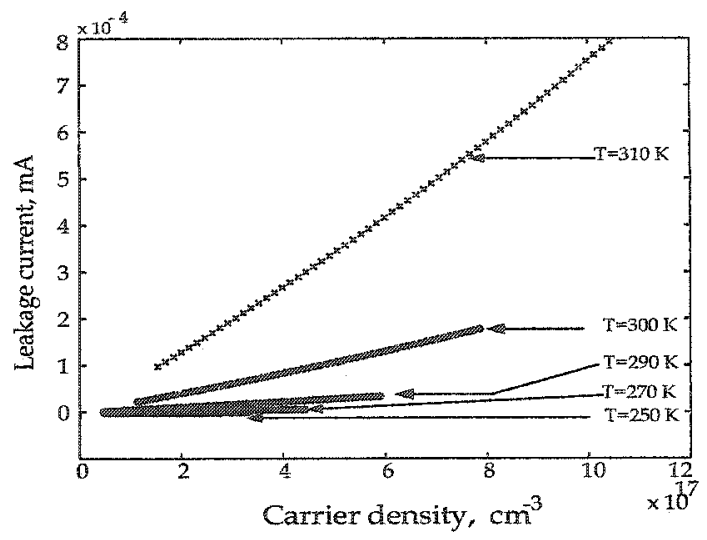

Figure 3.14: Variation of the leakage current with carrier density and the effect of temperature at lower temperatures from $250 \mathrm{~K} 310 \mathrm{~K}$.

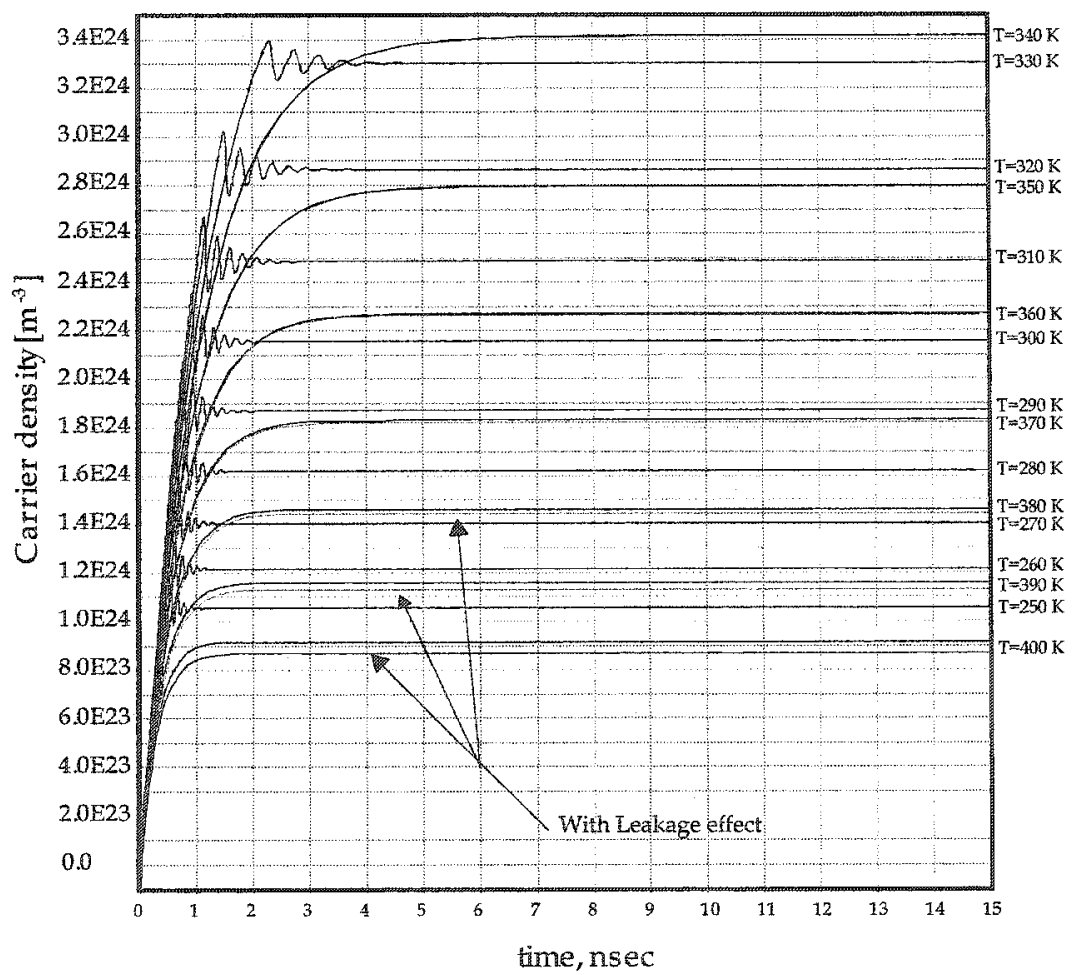

Figure 3.15: Time evolution of the carrier density. Temperature varied between 250 K and 400 K. Simulations were performed with and without including the effect of the leakage current $[7]$. 

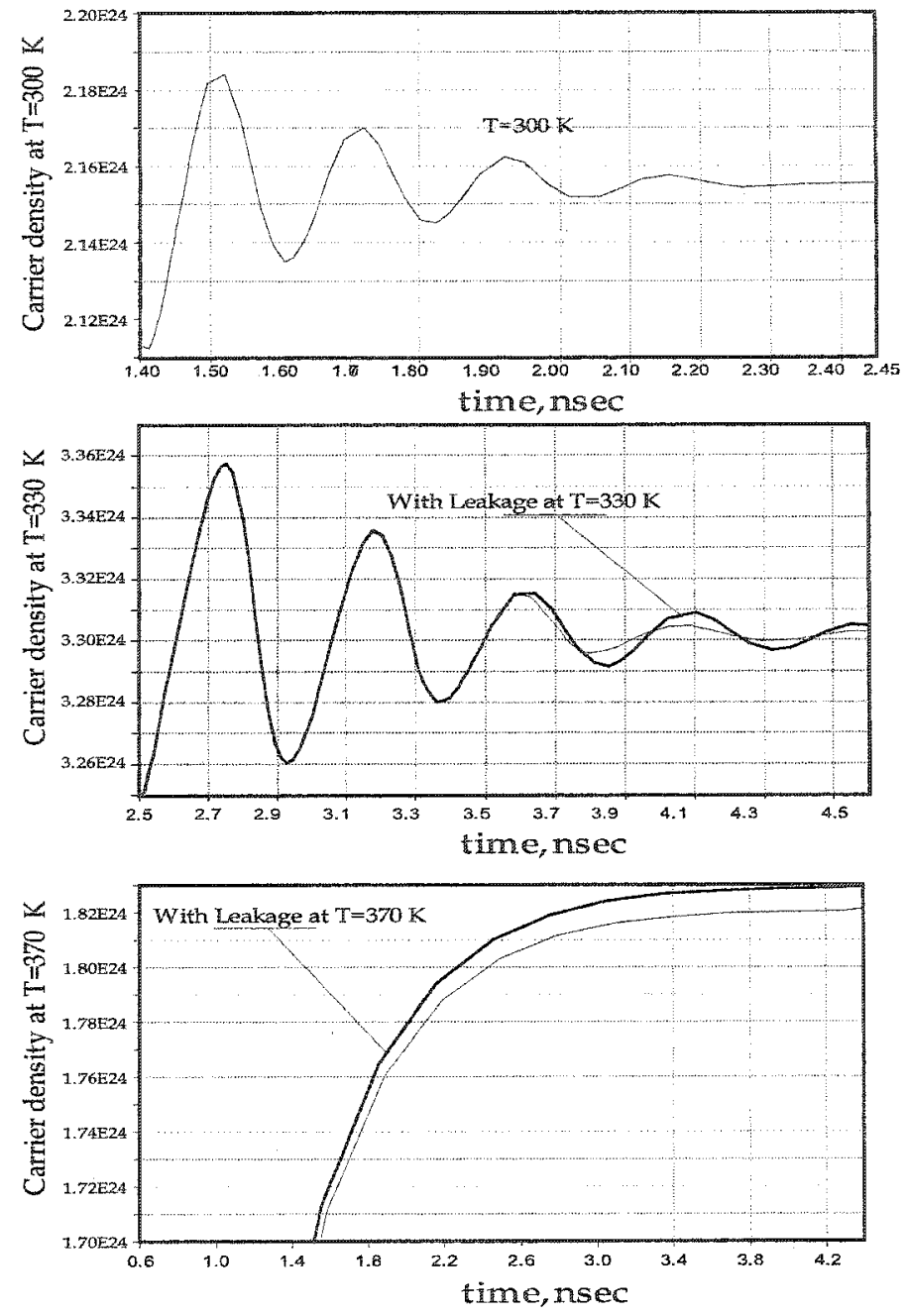

Figure 3.16: Time evolution of the carrier density at temperatures $300 \mathrm{~K}, 330 \mathrm{~K}$ and $370 \mathrm{~K}$ with and without including the effect of the leakage current.

The effect of the leakage current on the carrier density is noticeable at high temperatures, as shown in Figure 3.16. The presented leakage current model is based on the diffusion theory of barrier injection, in which the barrier height depends on the acceptor concentration in the p-cladding layer, and on the carrier mobility. An interesting point is that the doping levels can be tuned along with the laser geometry and used as controlling parameters to minimize the leakage current, leading to an increase in the device efficiency. This concept 
has been experimentally proven [139].

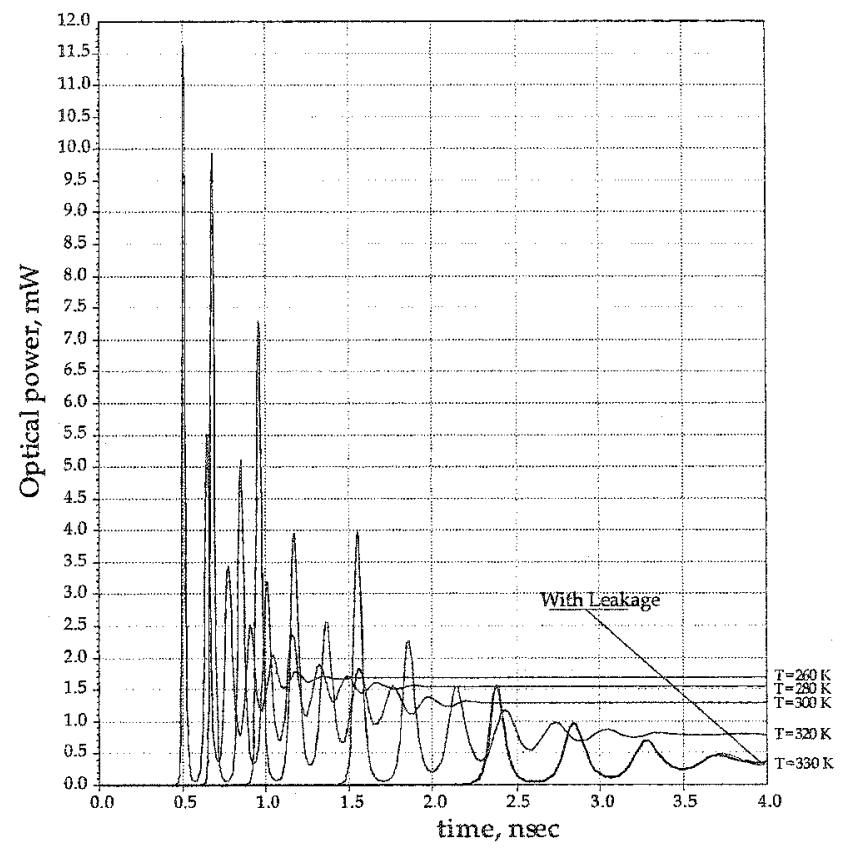

Figure 3.17: Time evolution of the optical power at temperatures $300 \mathrm{~K}, 330 \mathrm{~K}$ and $370 \mathrm{~K}$ with and without including the effect of the leakage current [2].

\subsection{Modified laser models including noise fluctuations}

Intensity and frequency noise produced by the laser diodes limits their use in optical communication and optical measuring [21,81]. Device and system performance evaluation and network characterization and improvements are the main reasons for studying and analyzing the laser intensity and frequency noise. Sophisticated mathematical models of laser noise have been introduced $[6,35,38,68,79,141,142]$, but they are not generally suitable for both device and systems simulations as proposed in $[2,3]$. Analytical laser noise models based on a small signal approximation of the laser rate equations driven by Langevin noise sources appear in the literature $[6,34-36,79]$, but the reported models does not include dynamic (instantaneous) fluctuation information, and they are not suitable for large signal 
fluctuation analysis. Several sophisticated computer simulations based on direct numerical integration of the laser rate equations including the effect of Langevin noise sources have been introduced [37-39], but the cross-correlation relations between carriers, photons, and phase have been largely neglected in these models. Circuit models $[21,40,67]$ have been used for the prediction of relative intensity noise (RIN), but again the cross-correlation relations were neglected, and the resulting models are not generally suitable for system simulations.

This section presents an extended analytical study for the auto and cross correlation relations between the noise sources. Using quantum mechanics and Quantum noise generation theory, the semiconductor laser rate equations were modified to include noise sources. The auto-correlation and the cross-correlation properties of the noise sources were incorporated into the derived rate equations. Details are given in Appendix $\mathbf{E}$. The derived expressions provide a theoretical base for the expressions presented by M. Ahmed et al. [81] and a generalization of Marcuse's noise analysis [68]. A self-consistent normalized laser model that includes the correlated noise sources is proposed.

\subsubsection{Noise driven enhanced laser model}

The normalized laser model given by (3.1.17) and (3.1.18), including the leakage current effect given in (3.2.5) may be modified by adding the phase rate equation and the effects of the noise sources as derived in Appendix E, leading to the normalized modified noise driven laser model, given by,

$$
\begin{aligned}
\frac{d \mathbb{P}_{n}(T, t)}{d t}= & \frac{\left(\mathbb{C}_{n}(T, t)-\mathbb{C}_{t r}^{n}(T)\right)}{\tau_{p h}(T)\left(1-\mathbb{C}_{t r}^{n}(T)\right)} \frac{\mathbb{P}_{n}(T, t)}{\sqrt{1+\mathbb{P}_{r}^{n}(T, t)}}-\frac{\mathbb{P}_{n}(T, t)}{\tau_{p h}(T)} \\
& +\left(\beta_{s p} B_{o} \tau_{e}(T) \mathbb{C}_{t h o} \exp \left(\frac{T-T_{r}}{T_{o}}-\frac{E 1 q}{K_{B} T}\right)\right) \mathbb{C}_{n}^{2}(T, t)+F_{\mathbb{P}_{n}}(t)(3
\end{aligned}
$$




$$
\begin{aligned}
\frac{d \mathbb{C}_{n}(T, t)}{d t}= & \frac{I_{e f f}(t)}{q V_{c} \mathbb{C}_{t n}(T)}-\frac{\left(\mathbb{C}_{n}(T, t)-\mathbb{C}_{t r}^{n}(T)\right)}{\tau_{e}(T) \tau_{p h}(T)\left(1-\mathbb{C}_{t r}^{n}(T)\right)} \frac{\mathbb{P}_{n}(T, t)}{\sqrt{1+\mathbb{P}_{r}^{n}(T, t)}} \\
& -\frac{\mathbb{C}_{n}(T, t)}{\tau_{e}(T)}+F_{\mathbb{C}_{n}}(t) \\
\frac{d \phi_{n}(t)}{d t}= & \frac{\beta}{2} G_{n}\left(\mathbb{C}_{n}(T, t)-\overline{\mathbb{C}}_{n}\right)+F_{\phi_{n}}(t)
\end{aligned}
$$

where $F_{\mathbb{P}_{n}}(t), F_{\mathbb{C}_{n}}(t), F_{\phi_{n}}(t)$ are the normalized photon, carrier, and phase Langevin noise sources, $\overline{\mathbb{C}}$ is the mean of the carrier number, $\beta$ is the linewidth enhancement factor and $G_{n}=\Gamma v_{g} \wp$

\subsubsection{Noise generation technique}

The stochastic functions $F_{\mathbb{P}_{n}}(t)$, and $F_{\phi_{n}}(t)$ in equations (3.3.1) and (3.3.2) may generated by a set of discrete-time Gaussian white noise sources that satisfy a multidimensional distribution with a probability density function $p d f(\underline{x})$ given as,

$$
p d f(\underline{x})=\frac{1}{\sqrt{\left((2 \pi)^{n}\|K\|\right)}} \exp \left(-(\underline{x}-\mu)^{T} K^{-1}(\underline{x}-\mu) / 2\right)
$$

in which $\underline{x}$ is a length $n$ vector, $K$ is the $n$-by-n covariance matrix, $\mu$ is the mean value vector, and the superscript $T$ indicates the matrix transpose. The covariance matrix $K$ is a diagonal matrix whose diagonal elements ${ }^{1} \mathfrak{R D}_{i i} x_{i}$ represent the variance in the number of events affecting $i$ in a time $\Delta t$. The set of jointly Gaussian random variables represent the changes in photons, carriers, and phase induced by the Langevin operators during the integration time step $\Delta t$. In order to obtain a suitable event state induced by the Langevin noise operator, the variables should be divided by the time interval between any two successive sampling times, $\Delta t$. Based on this discussion, a self-consistent technique for generating these stochastic functions is as follows:

* Generate three different white Gaussian random variable $x_{\mathbb{P}}, x_{\phi}$, and $x_{\mathbb{C}}$.

\footnotetext{
${ }^{1}$ The diagonal elements can be evaluated using equations (E.5.4) - (E.5.6), (E.5.8) and (E.5.9).
} 
* Generate the Langevin photons, phase and the new variable RVn noise operators as:

$$
\begin{aligned}
F_{\mathbb{P}_{n}}(t) & =\frac{\left.D_{\mathbb{P P}}\right|_{n}}{\sqrt{\Delta t}} x_{\mathbb{P}} \\
F_{\phi_{n}}(t) & =\frac{\left.D_{\phi \phi}\right|_{n}}{\sqrt{\Delta t}} x_{\phi} \\
F_{R V n}^{n}(t) & =\sqrt{\frac{\left.D_{n^{\prime} n^{\prime}}\right|_{n}}{\Delta t} x_{\mathbb{C}}}
\end{aligned}
$$

* Generate the carriers noise source using $F_{\mathbb{C}_{n}}(t)=F_{R V n}^{n}(t)-\left(\mathfrak{R}_{\mathbb{P} \mathbb{C}}^{n} F_{\mathbb{P}_{n}}+\mathfrak{R}_{\mathbb{C} \phi}^{n} F_{\phi_{n}}\right)$. The $\left.\right|_{n}$ means the normalized form of the operator, $\mathfrak{R}_{\mathbb{P}}^{n}, \mathfrak{R}_{\mathbb{C} \phi}^{n}$ are the normalized cross-correlated operators.

\subsubsection{Enhanced SDD model implementation}

A semiconductor laser model as defined by (3.3.1) - (3.3.3) was implemented using Symbolically Defined Devices (SDDs) in Agilent-ADS. The three-port SDD laser model proposed in Section 3.1.2 and shown in Figure 3.1 was enhanced by adding an extra port for implementing the phase rate equation. The implemented model is suitable for both small and large signal simulations in the time and frequency domains. Figure 3.18 shows the schematic symbol for the four-port SDD used to implement the normalized modified laser rate equations, while the experimental setup used for time domain simulations is illustrated in Figure 3.19.

\subsubsection{Simulation results}

Realistic laser diode parameters obtained from $[6,21]$ for InGaAsP semiconductor lasers and used in the simulations are listed in Tables 2.1 and 3.1. The proposed model shown in Figure 3.18 was used as a part of the experimental setup shown in Fig. 3.19 to predict the noise effects on the instantaneous values of carriers, photons, power, and phase $[4,8]$. The fixed simulation time steps were chosen to ensure white Gaussian noise generation while 


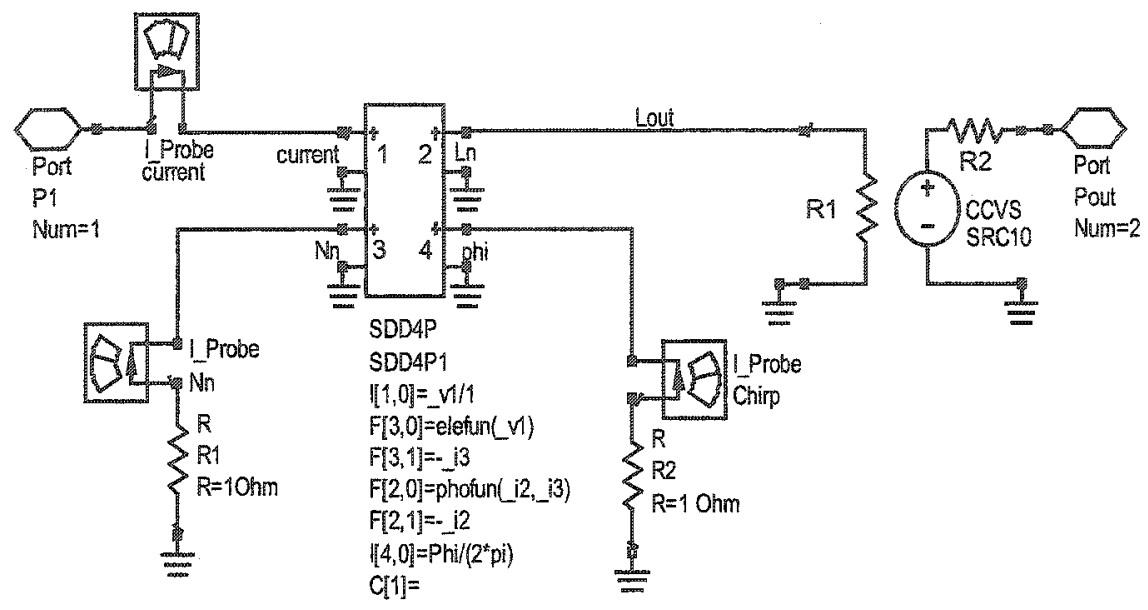

Parameters, Constants, and Calculations

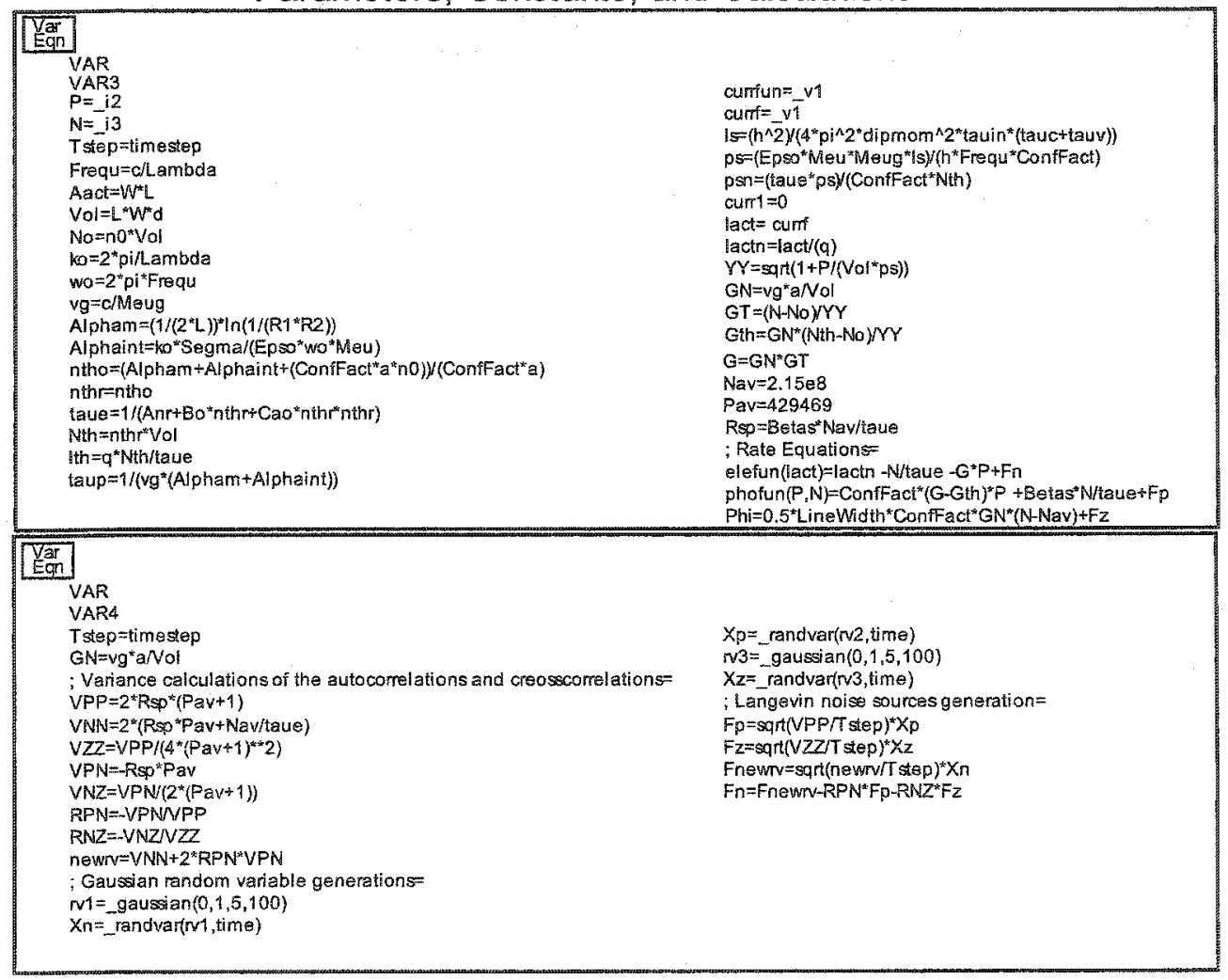

Figure 3.18: Schematic symbol for the four port SDD model.

preserving reasonable simulation time. The largest time step used was $\Delta t=5$ ps which corresponds to a $200 \mathrm{GHz}$ noise spectrum. This bandwidth is much larger than the laser's 


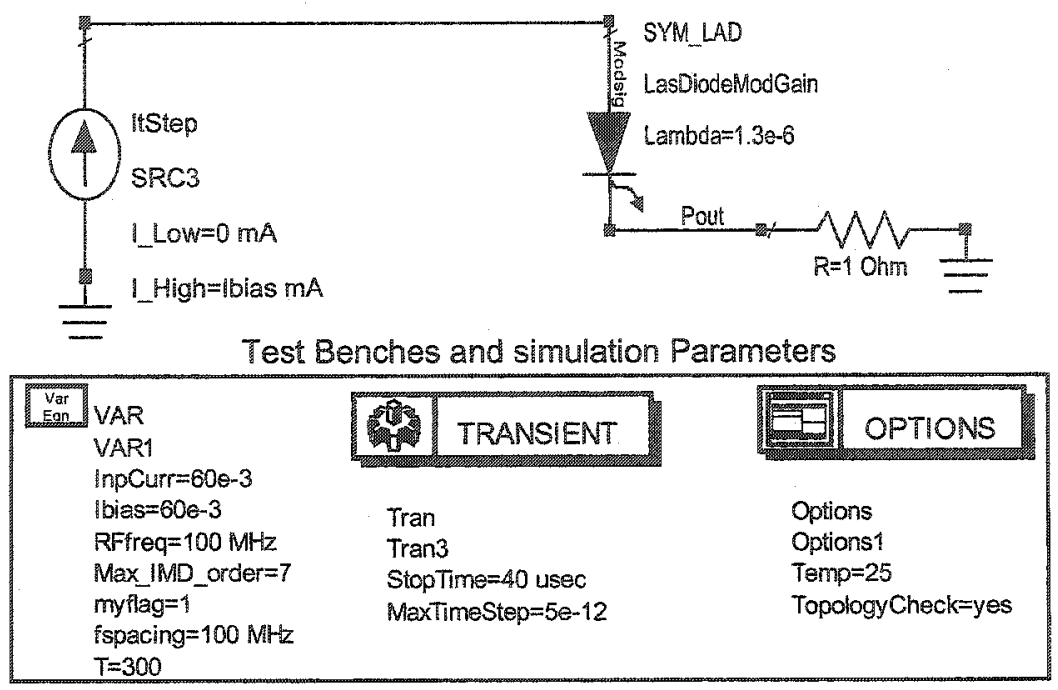

Figure 3.19: Agilent-ADS schematic diagram for the simulation setup. The diode symbol shown represents the hierarchically defined SDD shown in Figure 3.18. The output power is represented by a current in this simulation.

relaxation oscillation frequency (6 GHz at a $60 \mathrm{~mA}$ bias level as shown in Figure 2.15 [3]).

\subsubsection{Carrier, photon, and power results}

The time evolution of carrier number, photon number, and optical power output - with and without the effect of the noise - including the start-up transient period are shown in Figures 3.20, 3.22 and 3.24. The corresponding steady state conditions are shown in Figures 3.21, 3.23 and 3.25. From these figures, it is readily seen that adding the noise operators causes the fluctuation of the carriers, photons, and power around their steady state values during and after the transient period. The effect of noise is less visible during the start-up transient, due to the relative scale of the transient conditions and the noise. The steady state solution as shown in Figures $3.21,3.23$ and 3.25 agrees with the small signal results using the perturbation theory given by [6]. 


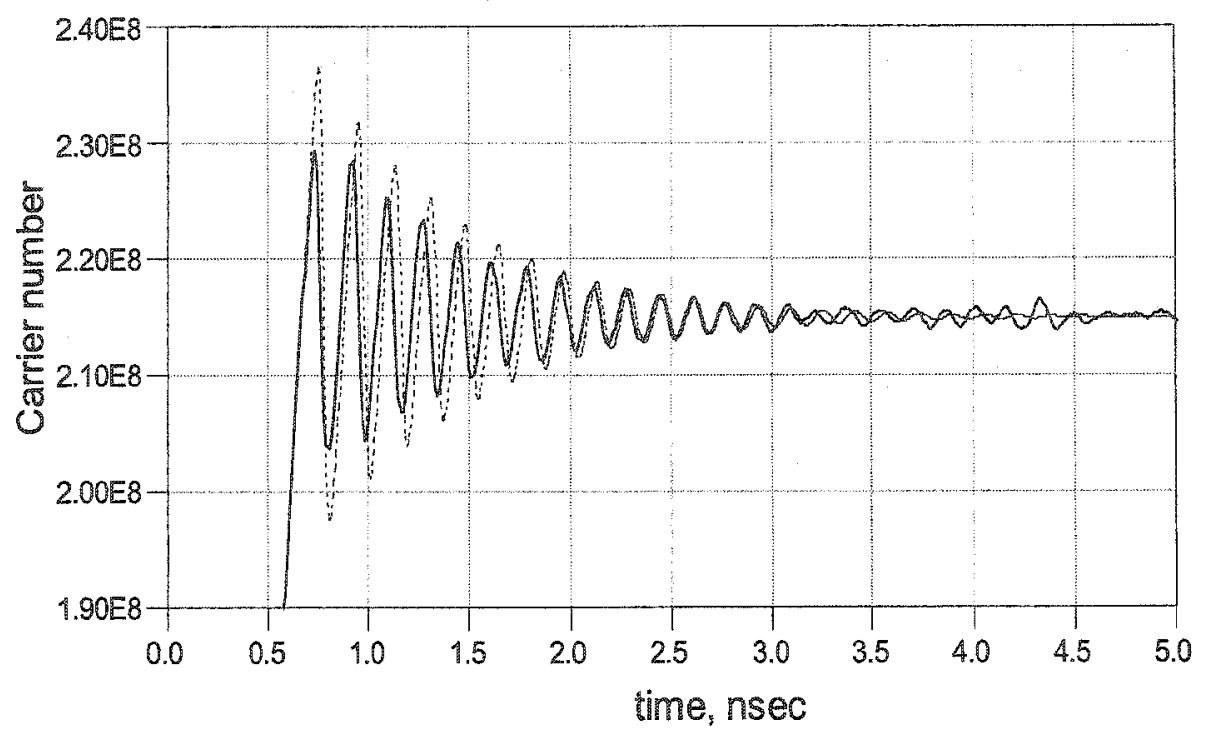

Figure 3.20: Time evolution of the carrier number with the effect of noise operators (solid lines) and without the effect of noise operators (doted lines) including transient [8].

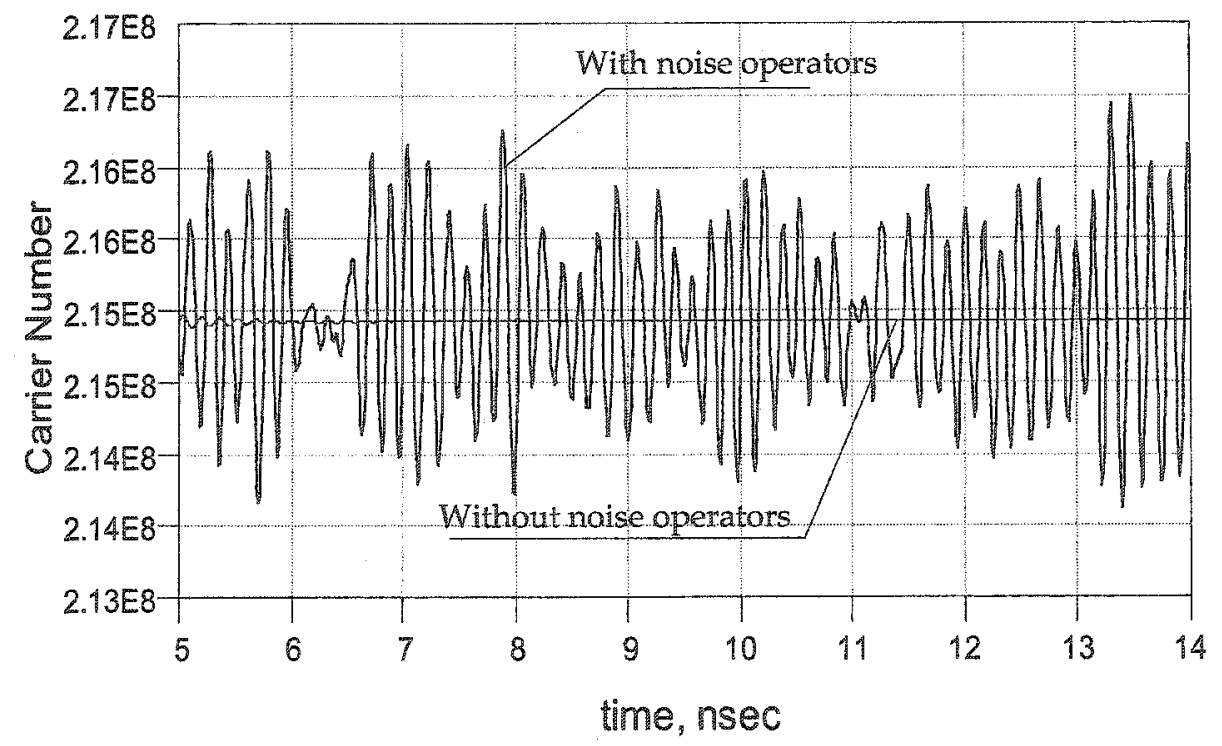

Figure 3.21: Time evolution of the carrier number with and without the effect of noise operators for steady state operation [8]. 


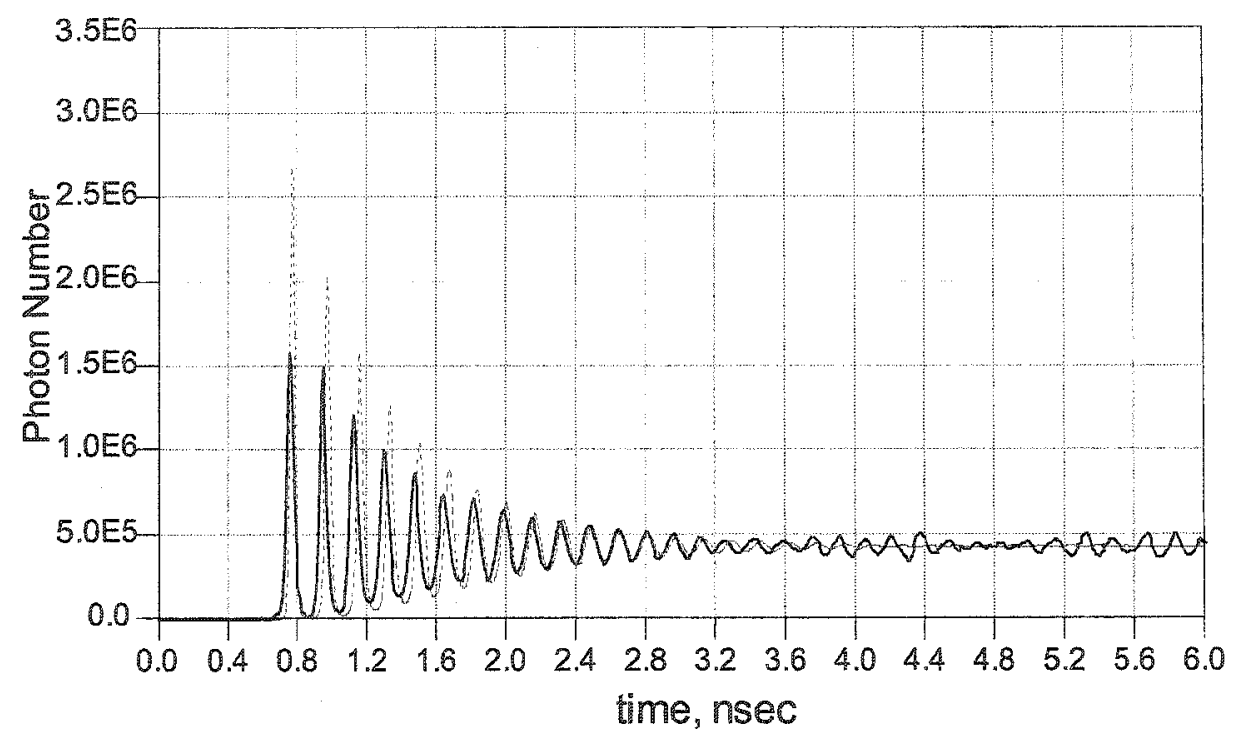

Pigure 3.22: Time evolution of the photon number with the effect of noise operators (solid lines) and without the effect of noise operators (doted lines) including transient [8].

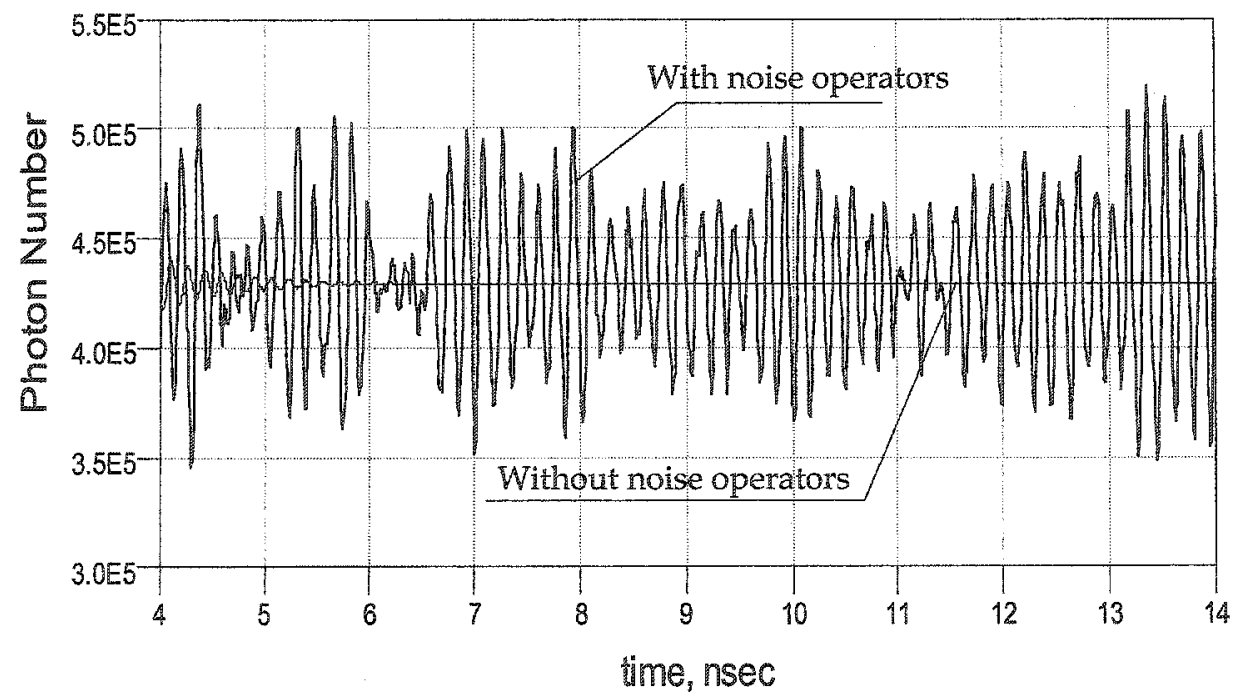

Figure 3.23: Time evolution of the photon number with and without the effect of noise operators for steady state operation [8]. 


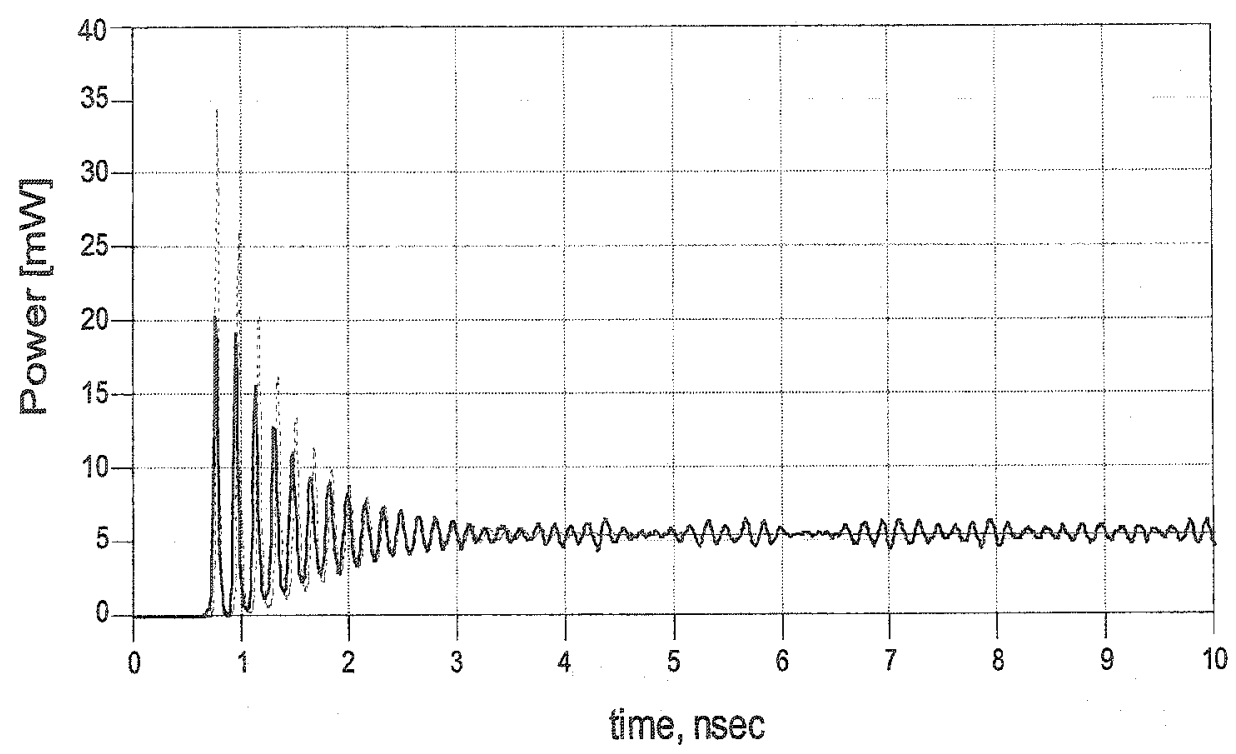

Figure 3.24: Time evolution of the output power with the effect of noise operators (solid lines) and without the effect of noise operators (doted lines) including transient [8].

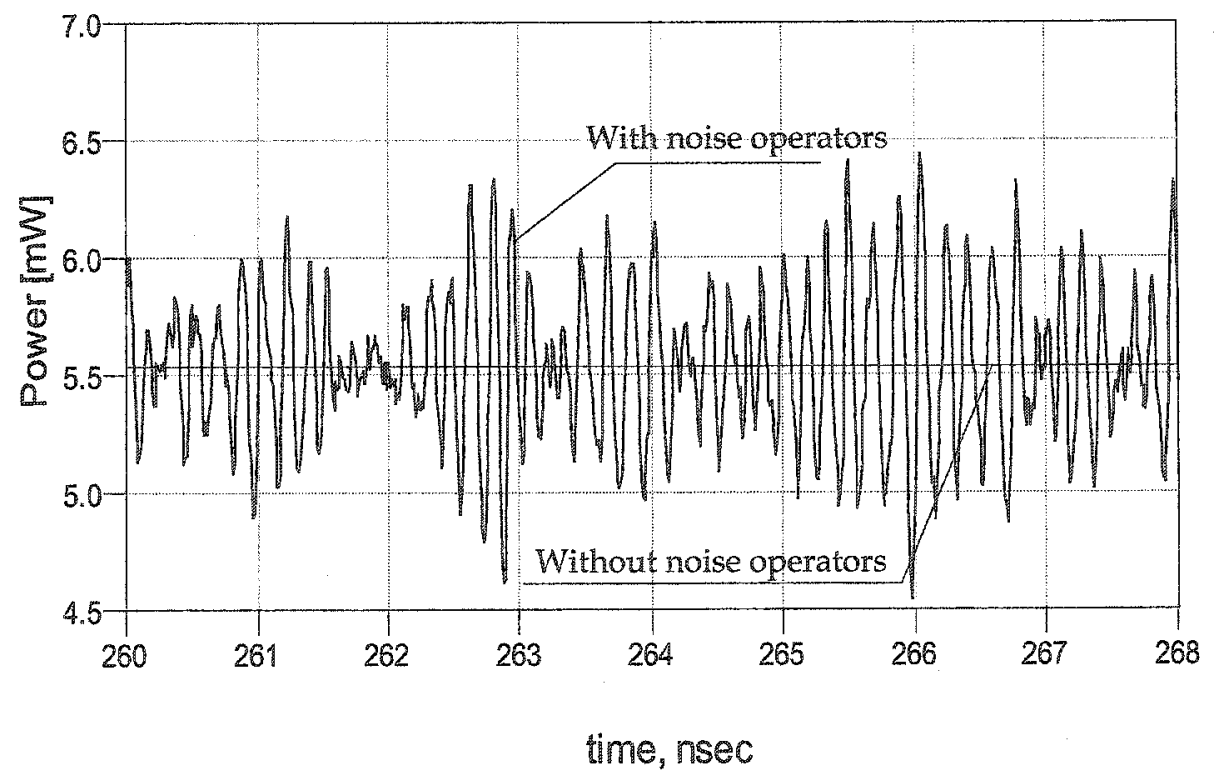

Figure 3.25: Time evolution of the output power with and without the effect of noise operators for steady state operation [8]. 


\subsubsection{Laser intensity noise}

Laser intensity noise is characterized by relative intensity noise (RIN). RIN may significantly degrad the system carrier-to-noise ratio (CNR), so it is important to study its effect on link performance. RIN is defined as the ratio of the spectral density of the fluctuated power to the steady state mean square power, and is given by,

$$
\operatorname{RIN}(\omega)=\frac{\mathbb{P}_{S}(\omega)}{\overline{\mathbb{P}}^{2}}=\frac{\left\langle|\mathbb{P}(\omega)|^{2}\right\rangle}{\overline{\mathbb{P}}^{2}}
$$

in which $\mathbb{P}_{S}(\omega)$ is the spectral density (ensemble average) given by,

$$
\mathbb{P}_{S}(\omega)=\int_{0}^{\infty} \delta \mathbb{P}(t) \delta \mathbb{P}(t+\tau) e^{-j \omega \tau} d \tau
$$

where $\delta \mathbb{P}(t)=(\mathbb{P}(t)-\overline{\mathbb{P}}), \overline{\mathbb{P}}$ is the average number of photons, and $\mathbb{P}(\omega)$ is the Fourier transform of $\delta \mathbb{P}(t)$ and given as,

$$
\mathbb{P}(\omega)=\int_{0}^{\infty} \delta \mathbb{P}(\tau) e^{-j \omega \tau} d \tau
$$

Based on our simulations, the calculated time average of the number of photons over 25 runs with different seeds is equal to the statistical average over the same number. Therefore this process can be considered as an ergodic stochastic process [143-146]. Since this is an ergodic stochastic process, the ensemble average in Equation (3.3.7) can be replaced by the time average over a long time period T. Thus, the RIN is given as,

$$
R I N(\omega)=\frac{1}{\overline{\mathbb{P}}^{2}}\left\{\frac{1}{T} \int_{0}^{T}|\mathbb{P}(\omega)|^{2} d t\right\}
$$

Figure 3.26 shows the simulated RIN spectra and Figure 3.27 illustrates the results of the calculated RIN derived from the small signal analytical solution of the laser rate equations. From these two figures the laser RIN is maximum near the threshold which is also around the relaxation oscillation frequency $(6 \mathrm{GHz})$ based on the available laser parameters. RIN drops off from the peak located just above this frequency. As shown in Figure 3.26, the 
RIN is nearly flat (white) at low frequencies above $10 \mathrm{KHz}$. Below $10 \mathrm{KHz}$, RN has a 1/f characteristic. As shown in Figure 3.27, increasing the injection current increases the output optical power leading to a decrease in the RIN amplitude and a shift of the peak frequency of the RIN toward the higher frequency.

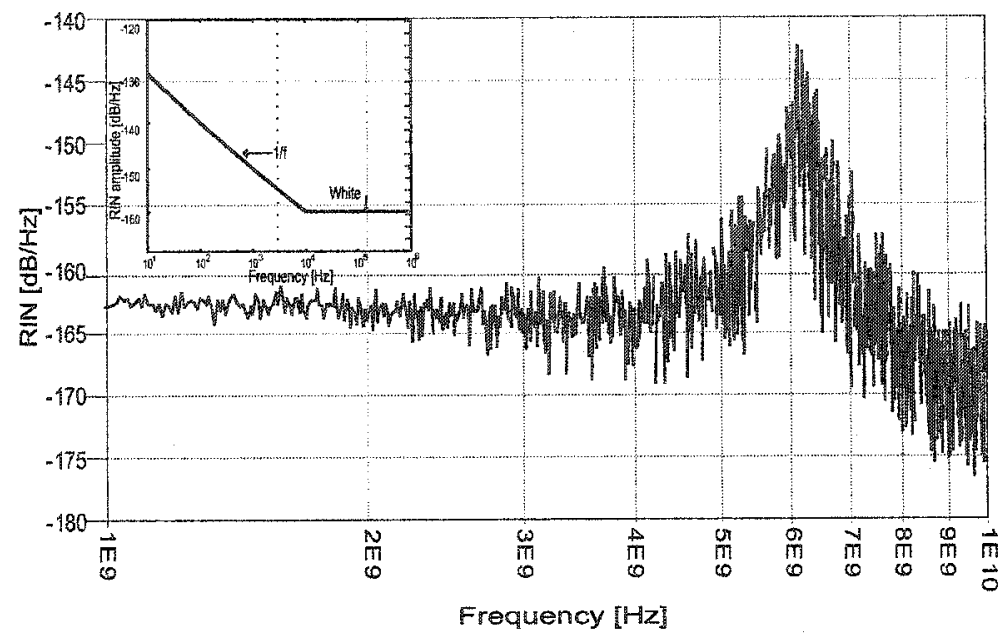

Figure 3.26: Laser diode relative intensity noise spectra (RIN) and frequency dependance of the amplitude noise [4].

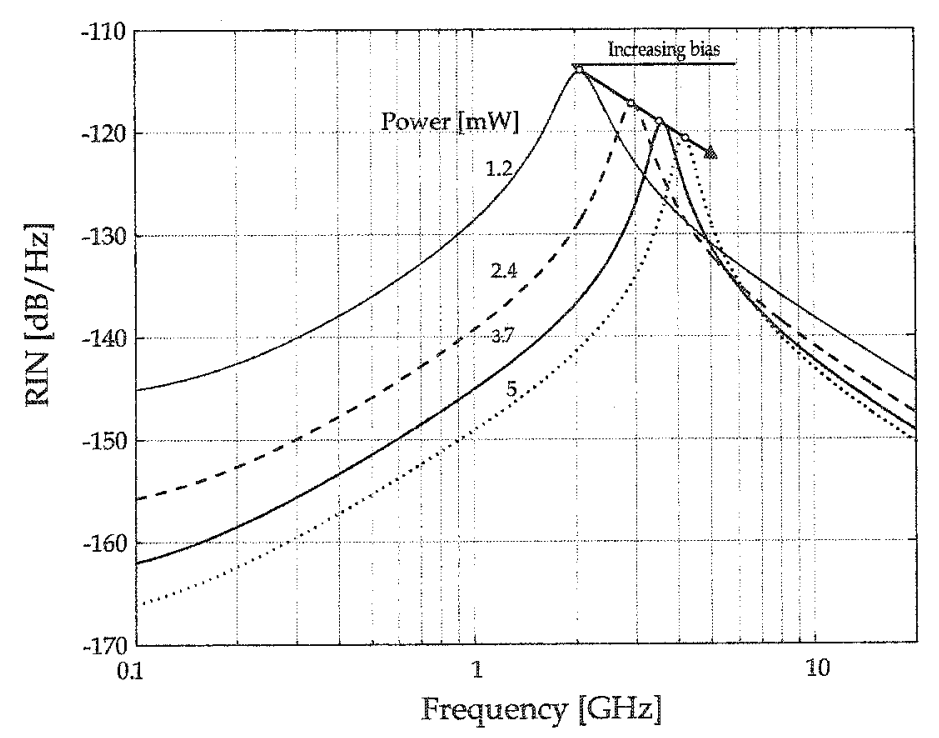

Figure 3.27: Intensity noise spectra at several power levels showing the shift of the peak frequency as the bias current increases. 


\subsubsection{Laser phase/frequency noise}

Phase noise is an important concern in coherent optical communication systems and interferometer sensors. The magnitude of the noise depends on the laser's structure, material, and operating characteristics. Phase noise, $F N S_{\phi} .(\omega)$, is defined by the spectral density of the frequency noise and given by,

$$
\begin{aligned}
& F N S_{\phi}(\omega)=\left\langle|\omega \Delta F(\omega)|^{2}\right\rangle \\
& F N S_{\phi} \cdot(\omega)=\int_{o}^{\infty} \Delta F(t) \Delta F(t+\tau) e^{-j \omega \tau} d \tau
\end{aligned}
$$

in which $\omega$ is the Fourier angular frequency and $\Delta F(\omega)$ is the Fourier transform of the time domain frequency noise $\Delta F(t) . \Delta F(t)$ is the frequency fluctuation around the steady state frequency and given by,

$$
\Delta F(t)=\frac{1}{2 \pi} \frac{d \phi(t)}{d t}
$$

Phase noise leads to a frequency shift which is interpreted as laser line width broadening. The phenomenon of the dynamic shift of the lasing frequency is know as frequency chirping. It is also known as wavelength chirping, since $\Delta \lambda(t)=\left(-\lambda^{2} / c\right) \Delta F(t)$. Since the frequency noise is an ergodic stochastic process, the ensemble average in Equation (3.3.11) can be replaced by time average taken over a long time period T. $F N S_{\phi} .(\omega)$ is then given by,

$$
F N S_{\phi}(\omega)=\frac{1}{T} \int_{0}^{T}\left|\Delta F(\tau) e^{-j \omega \tau} d \tau\right|^{2} d t
$$

Figure 3.28 demonstrates instantaneous frequency fluctuations including the transient response, and Figure 3.29 illustrates steady state operation, with and without including the noise operators in the model. As shown in these figures, the frequency noise is highest during the transient period, but it rapidly dampens and fluctuates around the steady sate with a nearly constant envelop. Figure 3.30 shows the phase noise characteristic of a laser diode. The frequency noise spectrum is relatively flat for low frequencies. It peaks at the 


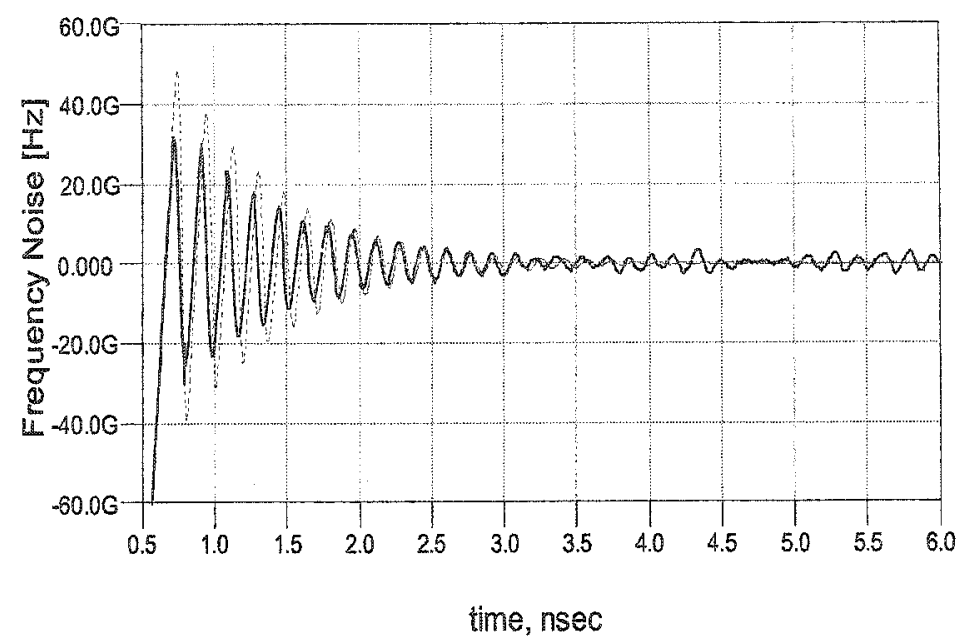

Figure 3.28: Time evolution of the phase with the effect of noise operators (solid lines) and without the effect of noise operators (doted lines) including transient [8].

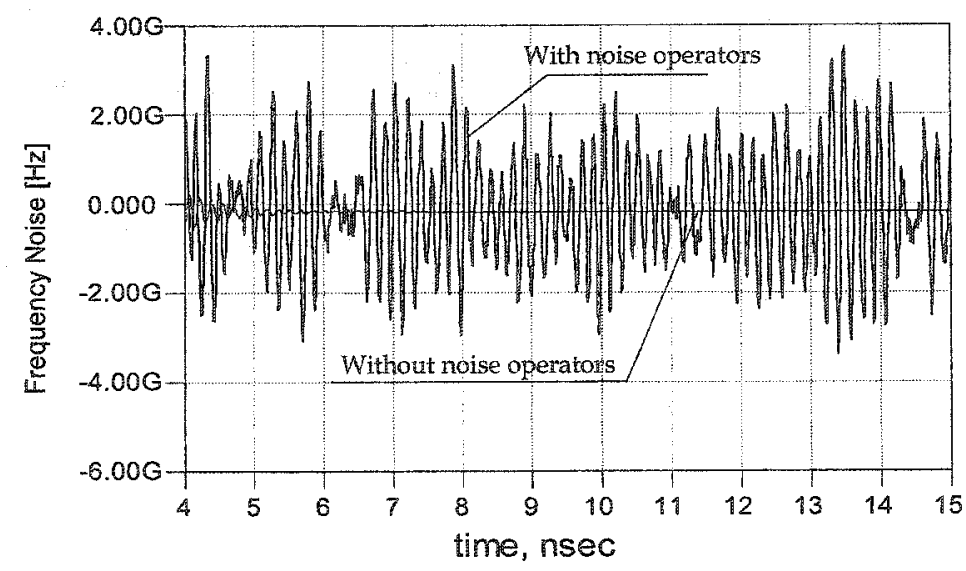

Figure 3.29: Time evolution of the phase with and without the effect of noise operators for steady state operation [8].

relaxation oscillation frequency. Frequency/phase noise simulations at very low frequencies are necessary for line width characterization, as line width is defined as,

$$
\Delta f=F N S_{\phi}(0) /(2 \pi)
$$

The results shown in Figure 3.30 are useful for determining the laser's line width. Generally, the line width decreases as the laser output power increases. For the laser considered 


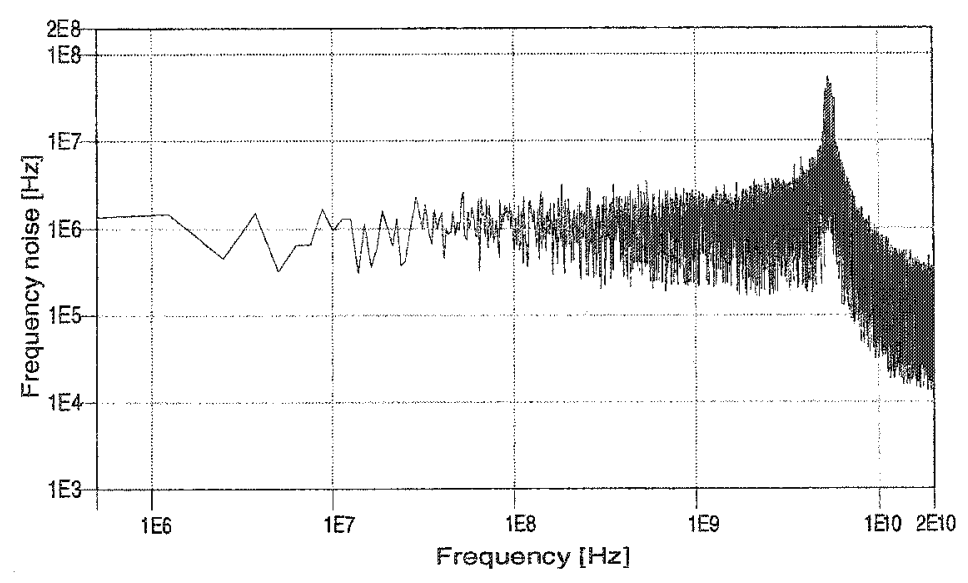

Figure 3.30: Laser diode phase/frequency noise spectra $[4,8]$.

in Figure 3.30, the line width is found to saturate to a value in the range 1-10 MHz at a power level above $10 \mathrm{~mW}$ [6]. At a $60 \mathrm{~mA}$ bias level, the line width is $\Delta F=13.5 \mathrm{MHz}$, which is comparable to published values $[6,21]$ and agrees with the measured results presented later in Chapter 4. Comparison of Figure 3.26 with Figure 3.30 demonstrates that the flat range of the frequency noise spectrum is larger than that of the intensity noise spectrum, but the general characteristics are similar.

\subsubsection{Summary and model advantages}

A computationally efficient model for analog and digital optical system simulations was presented. The reported model includes thermal effects, leakage current, and noise in an enhanced set of laser rate equations. An analog laser model was constructed using a symbolically defined device in Agilent-ADS. Time domain simulations of the enhanced laser model indicate that the laser carrier and photon density are strong decreasing functions of temperature. Simulations in time and frequency domains indicate that the modulation bandwidth and the relaxation oscillation frequency are also strongly decreasing functions of temperature. Noise effects were included in the model and allow for the simulation of RIN, FNS, and line width $\triangle f$. The RIN and the FNS were seen to have similar qualitative 
behavior and both are shaped by the laser irequency response. Models intended for analog optical transmission need to be suitable for system simulations in time and frequency domains and should include temperature, leakage, and noise effects. The proposed model and simulator implementation is the first such model that includes these effects simultaneously in a computationally attractive framework. The proposed model may therefore provide a useful mechanism for digital and analog optical link simulations in both the time and frequency domains. 


\section{Chapter 4}

\section{Experimentation and Model Verification}

In this chapter, the experimental results of the DC measurements, modulation response, distortion and noise of a directly modulated semiconductor laser are demonstrated. Also, comparison between the simulation and measurement results to validate the proposed model is presented.

\subsection{DC measurements}

The measurement of the output optical power is important for the determination of the power required for a fiber-optic link. The photograph of the experimental setup shown in Figure 4.1 is used for the DC measurement. The detailed block diagram of the experimental setup and the description of the measurement technique are included in Section G.1 of Appendix $G$. The measured output optical power versus drive current at room temperature $(27 C)$ for a $1.3 \mu \mathrm{m}$ InGaAsP-InP (Fujitsu FLD3F7CZ) butterfly packaged semiconductor laser is shown in Figure 4.2. From Figure 4.2, the lasing threshold current at room temperature is approximately $8 \mathrm{~mA}$. 


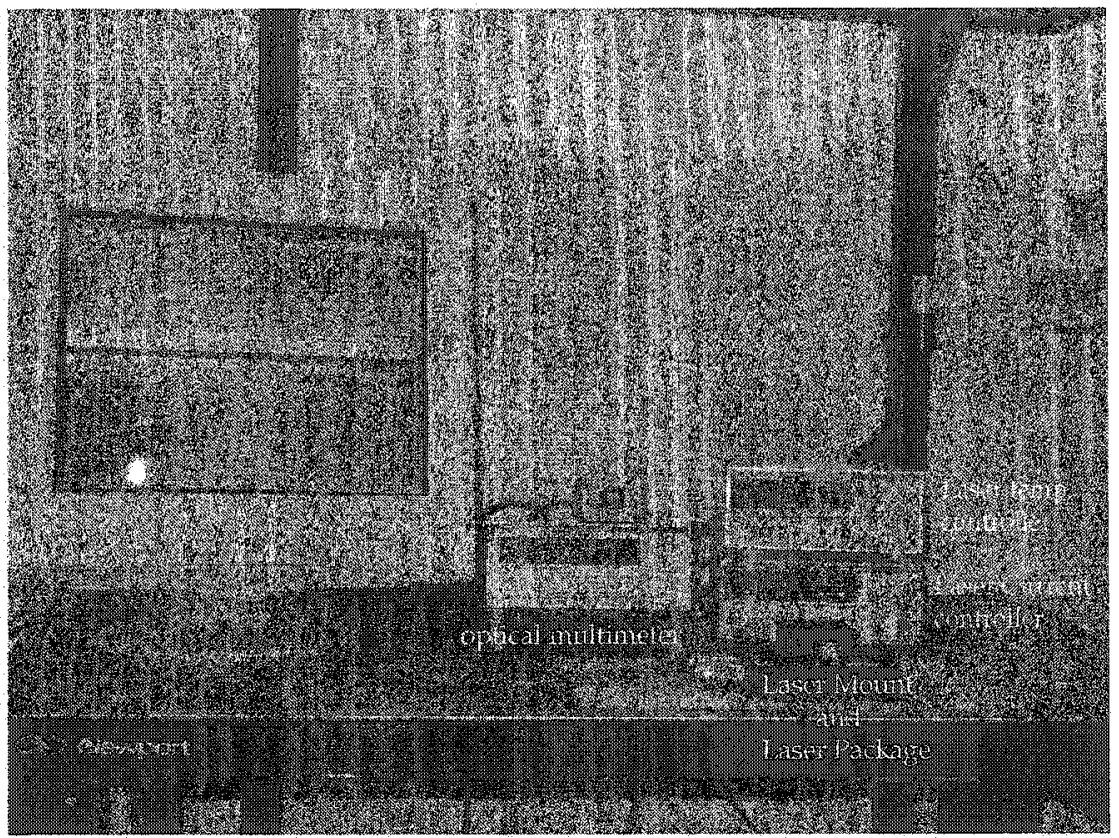

Figure 4.1: Experimental setup for the DC measurements.

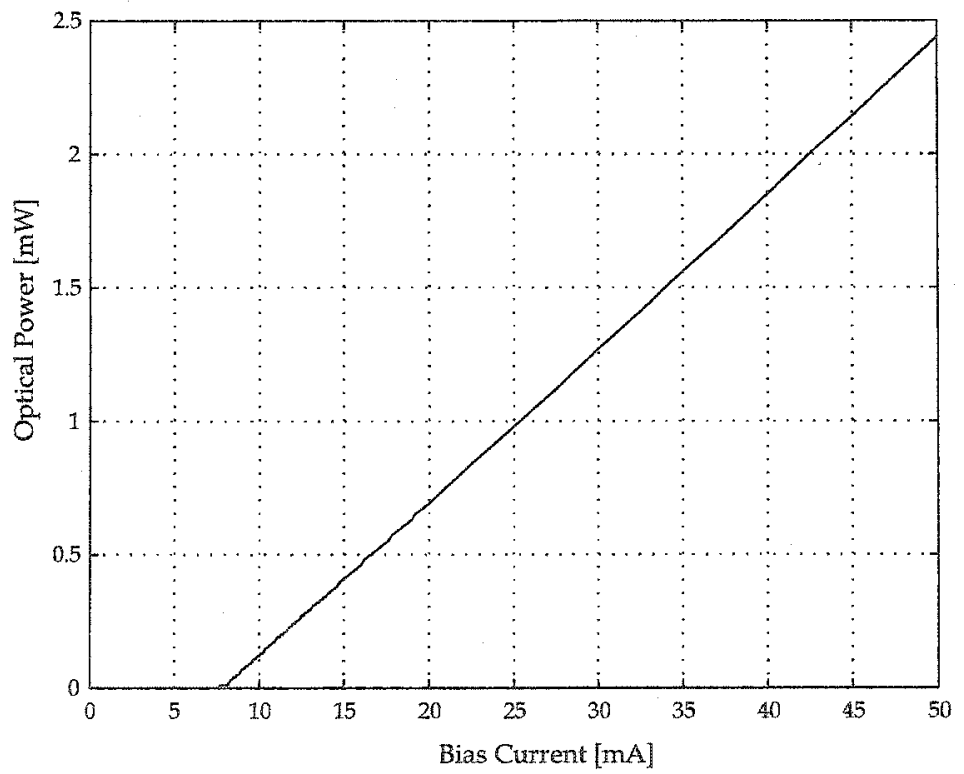

Figure 4.2: Output optical power versus driving current characteristics at room temperature for a $1.3 \mu \mathrm{m}$ InGaAsP-InP Fujitsu FLD3F7CZ butterfly packaged semiconductor laser. 


\subsection{Modulation response and linearity}

Modulation frequency response and linearity measurements are important in determining the modulation bandwidth and the link dynamic range. Modulation bandwidth is a limiting factor in the design of the analog optical links since it defines the usable frequency bandwidth of the whole link while the signal distortion due to nonlinearities decreases the total carrier-to-noise-ratio. In the next two sections, the modulation frequency response and linearity measurements were carried out at different frequencies using an Agilent $71400 \mathrm{C}$ light wave signal analyzer.

\subsubsection{Modulation frequency response}

The modulation frequency response of a semiconductor laser diode at different frequencies and different bias currents is measured using the experimental setup shown in Figure 4.3. The detailed block diagram of the experimental setup and the measurement description are included in Section G.2 of Appendix G.

Figure 4.4 shows the normalized modulation response of a $1.3 \mu \mathrm{m}$ InGaAsP-InP (Fujitsu FLD3F7CZ) laser diode at $27 C$ for different bias levels. The laser bias was swept from $10 \mathrm{~mA}$ to $30 \mathrm{~mA}\left(\simeq 3.75 I_{t h}\right)$, for a total of three bias levels. The input signal was a small signal sinusoid with input modulation frequency ranging from $0.5 \mathrm{GHz}$ to $10 \mathrm{GHz}$. From Figure 4.4 the relaxation frequency peaks are approximately at $2.2,6.2$ and $6.5 \mathrm{GHz}$ for bias levels of 10,20 , and $30 \mathrm{~mA}$ respectively. The laser modulation response curves for all bias levels indicate that the modulation response peak decreases monotonically with increasing laser bias current. The modulation bandwidth and relaxation oscillation frequency are increasing functions of bias current. The sharp cutoff frequency is mainly due to the product $\mathrm{RC}$ of the parasitic capacitance and series resistance of the laser diode. 


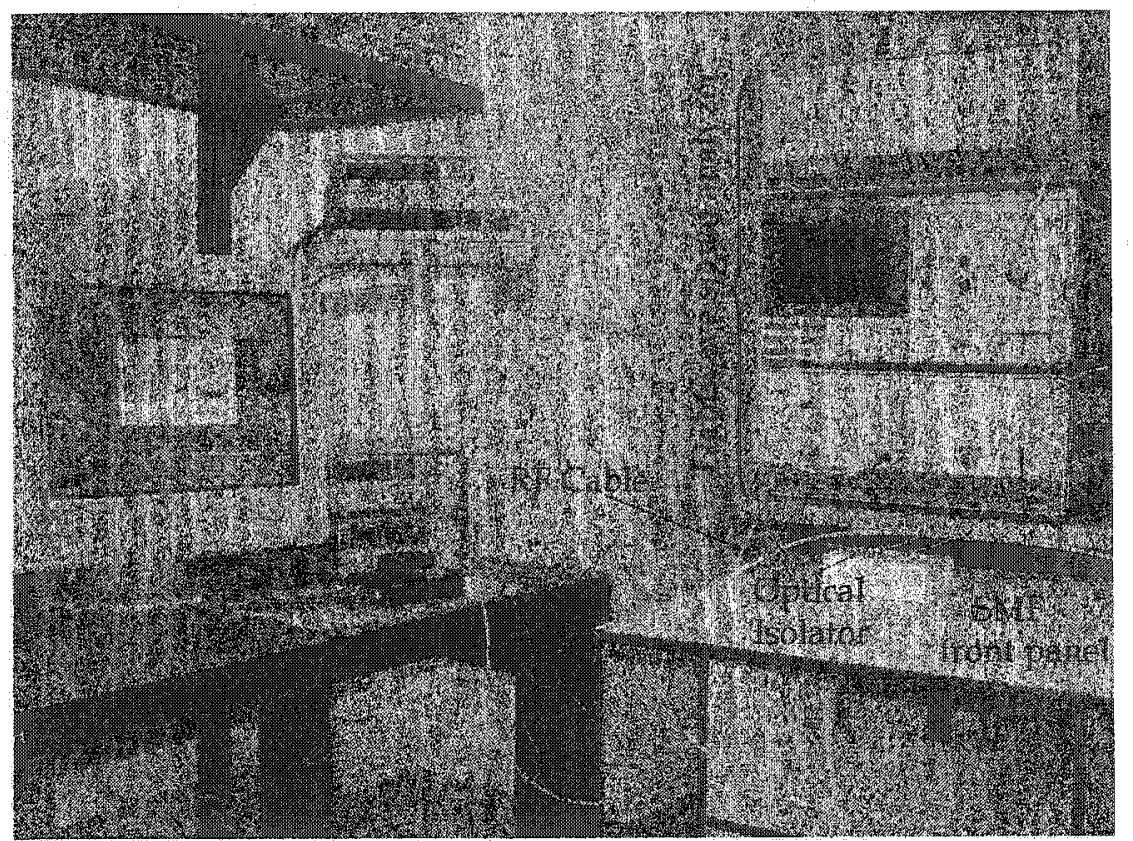

Figure 4.3: Experimental setup for the high frequency laser frequency response measurements.

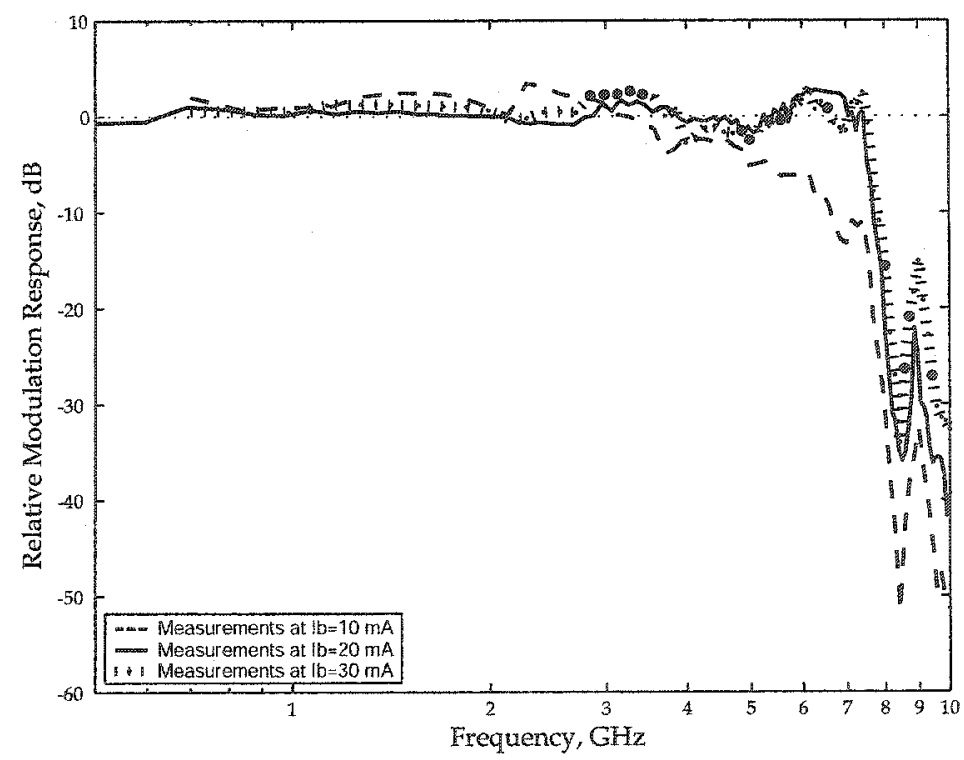

Figure 4.4: Modulation response of a $1.3 \mu \mathrm{m}$ InGaAsP-InP (Fujitsu FLD3F7CZ) laser at bias levels of 10,20 , and $30 \mathrm{~mA}$. 


\subsubsection{Distortion measurements}

Measurements of the laser nonlinearity are required to evaluate the performance of the system. In this section, the concept of measuring harmonic, second order and third order products using Agilent 71400C lightwave signal analyzer and multi-tone Agilent E4433B ESG-AP series signal generator is presented. The modulated light enters the light wave section in the $71400 \mathrm{C}$ system through a single-mode fiber (SMF) front-panel input connector $(\mathrm{FC} / \mathrm{PC})$. The optical power is detected, collimated and amplified, then focused onto InGaAs photodetector using the Agilent $70810 \mathrm{~B}$ lightwave section. The data was acquired through GPIB (IEEE488.2 interface) to a personal computer.

Different experiments under different conditions such as varying the frequency difference between carriers from $1 \mathrm{MHz}$ to $40 \mathrm{MHz}$, the carrier frequency up to $10 \mathrm{GHz}$, and power have been done for the purpose of this chapter, some of these experimental results are presented in the following sections.

\subsubsection{Harmonic response}

In this section, measurement results are presented for the harmonic distortion of a high speed laser diode 1.3um InGaAsP-InP (Fujitsu FLD3F7CZ) using the experimental setup shown in Figure 4.5. The detailed block diagram of the experimental setup and the measurement description are included in Section G.3 of Appendix G. In this experimental study, 0.5 and $1 \mathrm{GHz}$ carrier frequencies were used. The measurements were done at $300 \mathrm{~K}$ and repeated for carrier powers 0 and $-5 \mathrm{dBm}$ (peak-to-peak modulating current $I_{p p} \simeq 12.64$ and $7.11 \mathrm{~mA}$ respectively). The measured second order harmonic power as a function of the bias current is shown in Figures 4.6 and 4.7 at 0.5 and $1 \mathrm{GHz}$ modulating frequencies. 


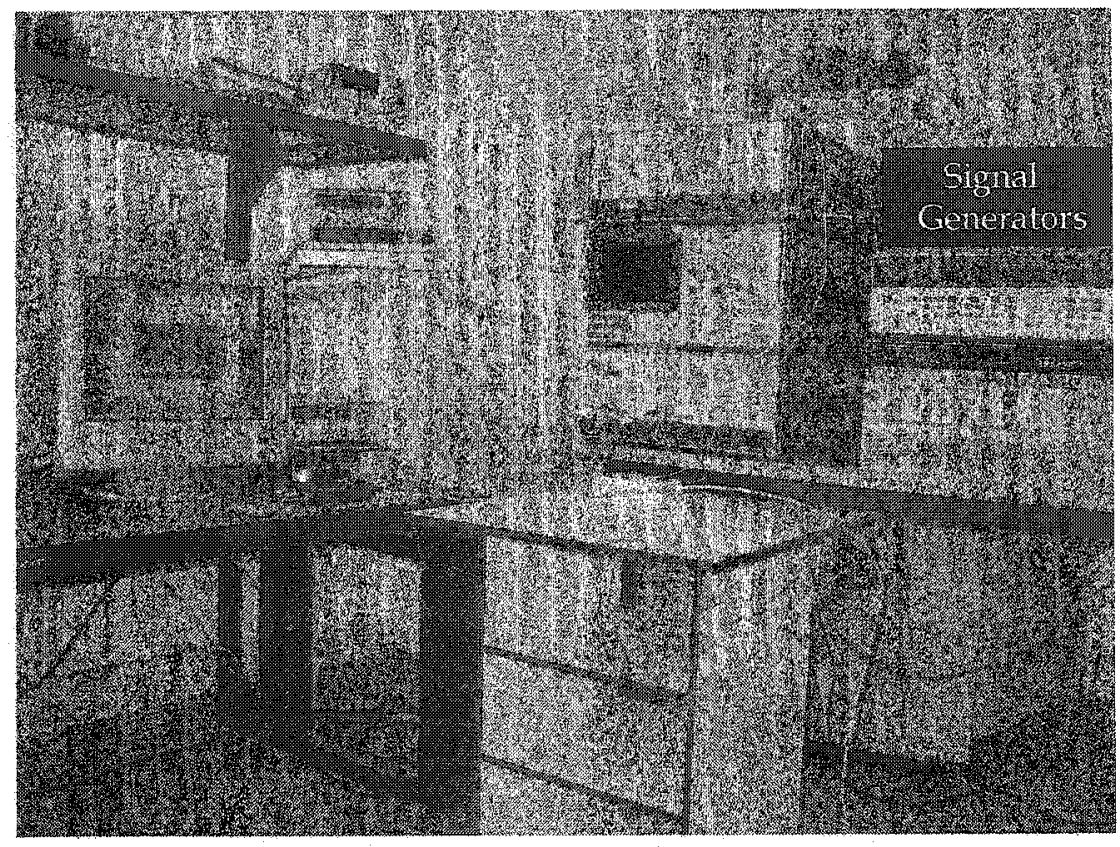

Figure 4.5: Experimental setup for the harmonic distortion measurements.

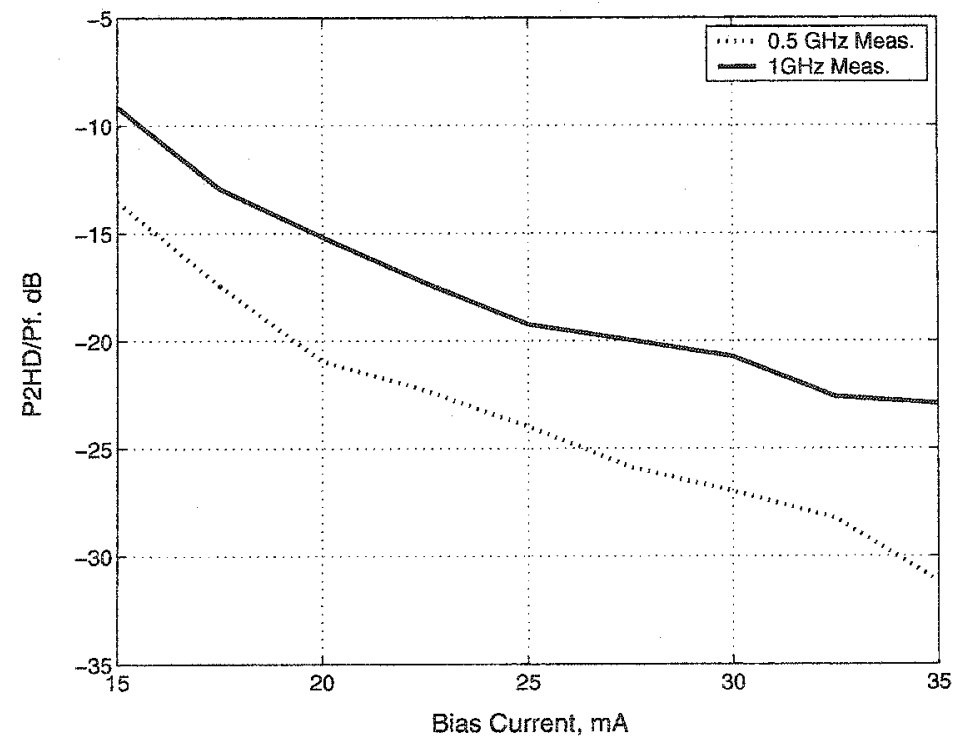

Figure 4.6: Measured power ratio of the second harmonic and the fundamental as a function of the bias current at carrier frequencies 0.5 and $1 \mathrm{GHz}$. The carrier power is $0 \mathrm{dBm}$. 


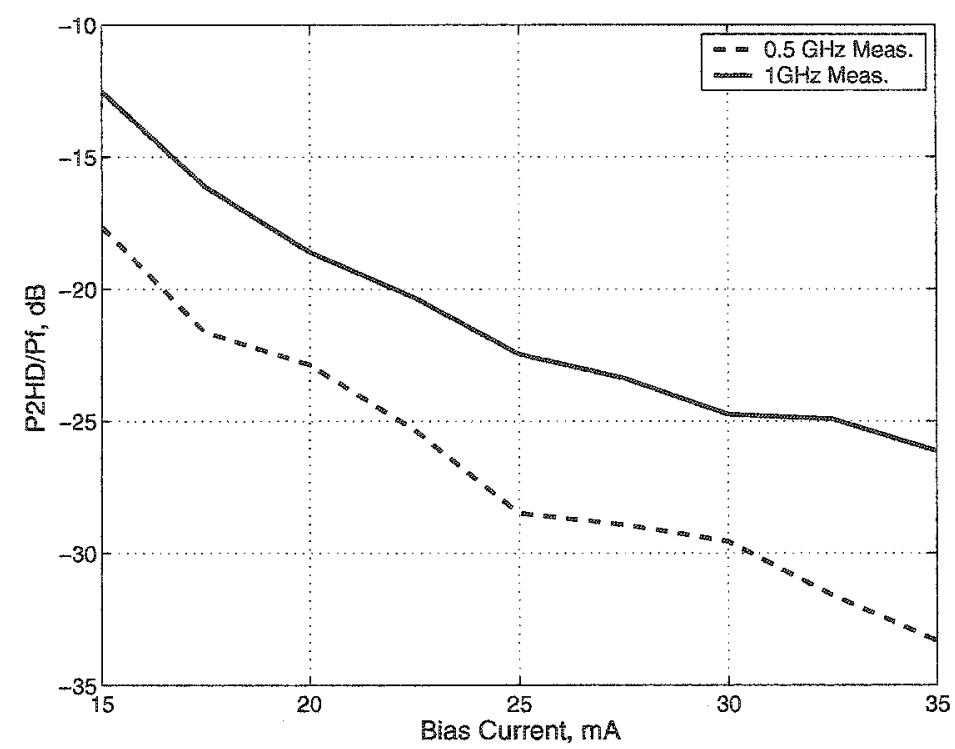

Figure 4.7: Measured power ratio of the second harmonic and the fundamental as a function of the bias current at carrier frequencies 0.5 and $1 \mathrm{GHz}$. The carrier power is $-5 \mathrm{dBm}$.

From Figures 4.6 and 4.7 it can be observed that the second harmonic distortion increases as the modulating frequency increases. The harmonic distortion is a decreasing function of the bias current for low and high frequencies. As the difference between the bias current and the threshold current decreases, the amplitude of the harmonic distortion becomes significant at low and high frequencies.

\subsubsection{Intermodulation distortion response}

Intermodulation distortion is a multi-tone distortion product that result when two or more signals are used to modulate the laser. In this experimental work two microwave signals with frequency separation of $40 \mathrm{MHz}$ are used to directly modulate a high speed laser diode 1.3 $\mu m$ InGaAsP-InP (Fujitsu FLD3F7CZ). The photograph of the experimental setup is shown in Figure 4.8. The detailed block diagram of the experimental setup and the measurement, description are included in Section G.3 of Appendix G.

In this experimental study, two signals of equal power and frequencies $f_{1}=0.98$ and $f_{2}=$ 
$1.02 \mathrm{GHz}$ were generated from a multi-tone Agilent E4433B ESG-AP series signal generator and applied to directly modulate the laser diode under test. The modulated output is then detected and focused onto InGaAs photodetector using Agilent $70810 \mathrm{~B}$ lightwave section. Agilent $71400 \mathrm{C}$ lightwave signal analyzer was used to perform the required measurements. The measurements were done at $300 \mathrm{~K}$ and repeated for different carrier powers. The measured second order intermodulation distortion power as a function of the bias current for signal powers 0 and $-2 \mathrm{dBm}\left(I_{p p} \simeq 12.64\right.$ and $\left.10.04 \mathrm{~mA}\right)$ at frequencies $f_{1}=0.98$ and $f_{2}=1.02 \mathrm{GHz}$ are shown in Figure 4.9. Using the experimental setup shown in Figure 4.8,

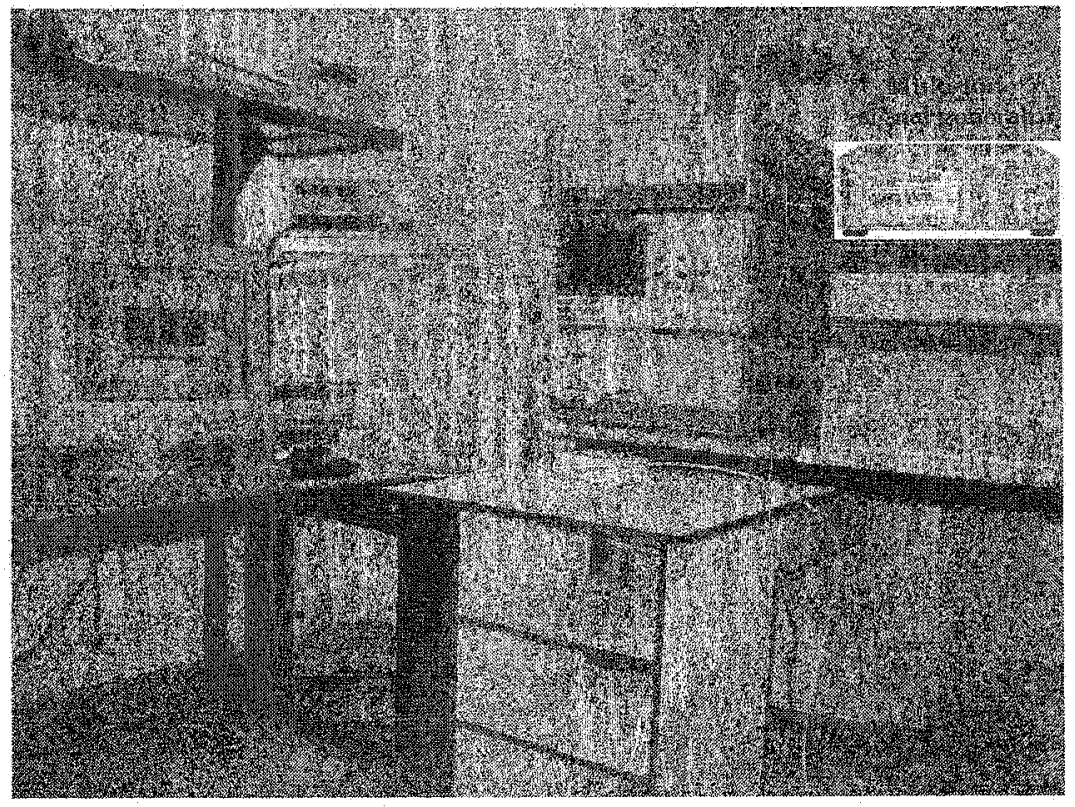

Figure 4.8: Experimental setup for intermodulation distortion measurements.

the desired third order intermodulation distortion products are acquired from the spectrum analyzer to a personal computer. The resulting power of the higher $\left(2 f_{2}-f_{1}\right)$ and lower $\left(2 f_{1}-f_{2}\right)$ third order intermodulation distortion power as a function of the bias current for signal powers 0 and $-2 \mathrm{dBm}\left(I_{p p} \simeq 12.64\right.$ and $\left.10.04 \mathrm{~mA}\right)$ at frequencies $f_{1}=0.98$ and $f_{2}=1.02 \mathrm{GHz}$ are shown in Figures 4.10 and 4.11 . 


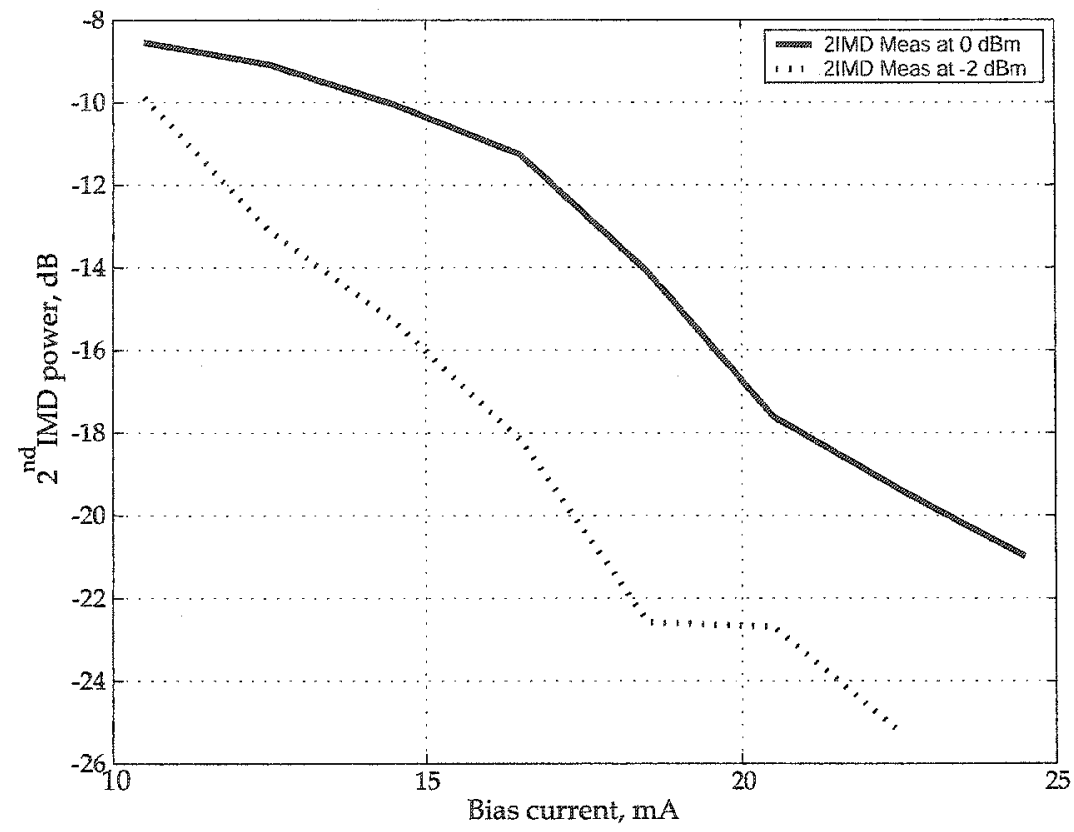

Figure 4.9: Measured second order intermodulation distortion power as a function of the bias current. The carrier frequencies are $f_{1}=0.98$ and $f_{2}=1.02 \mathrm{GHz}$.

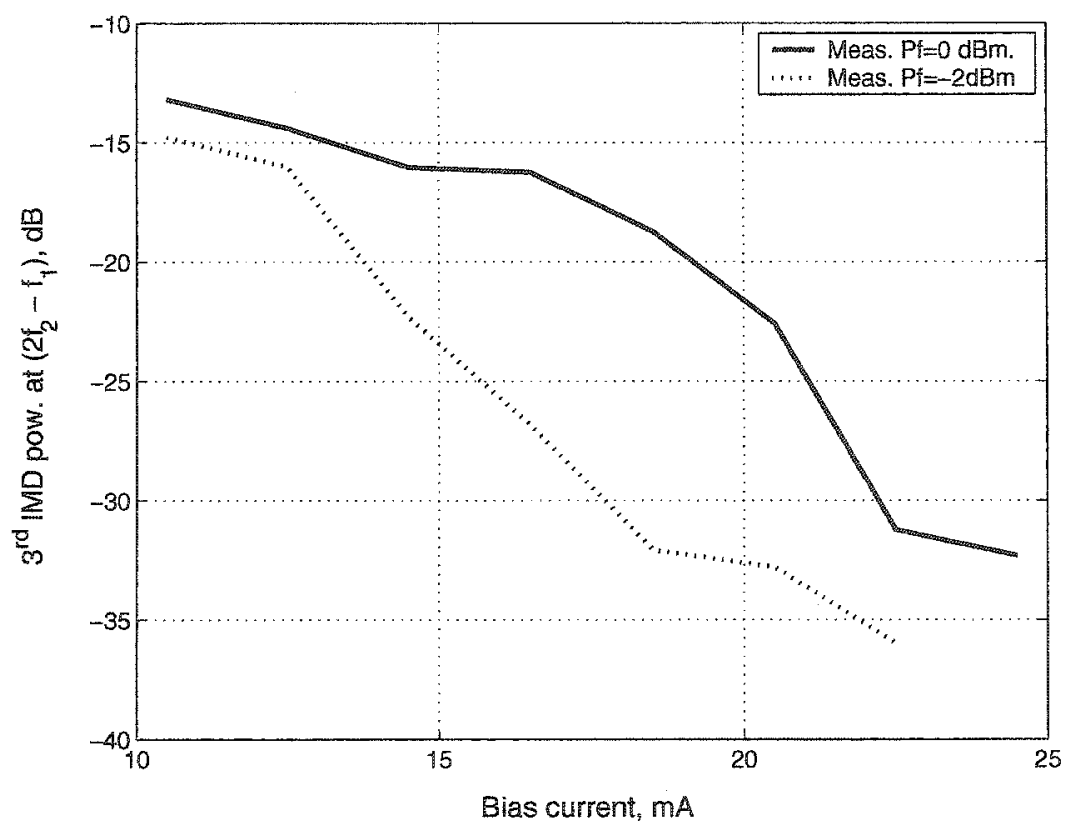

Figure 4.10: Measured higher $\left(2 f_{2}-f_{1}\right)$ third order intermodulation distortion as a function of the bias current. The carrier frequencies are $f_{1}=0.98$ and $f_{2}=1.02 \mathrm{GHz}$. 


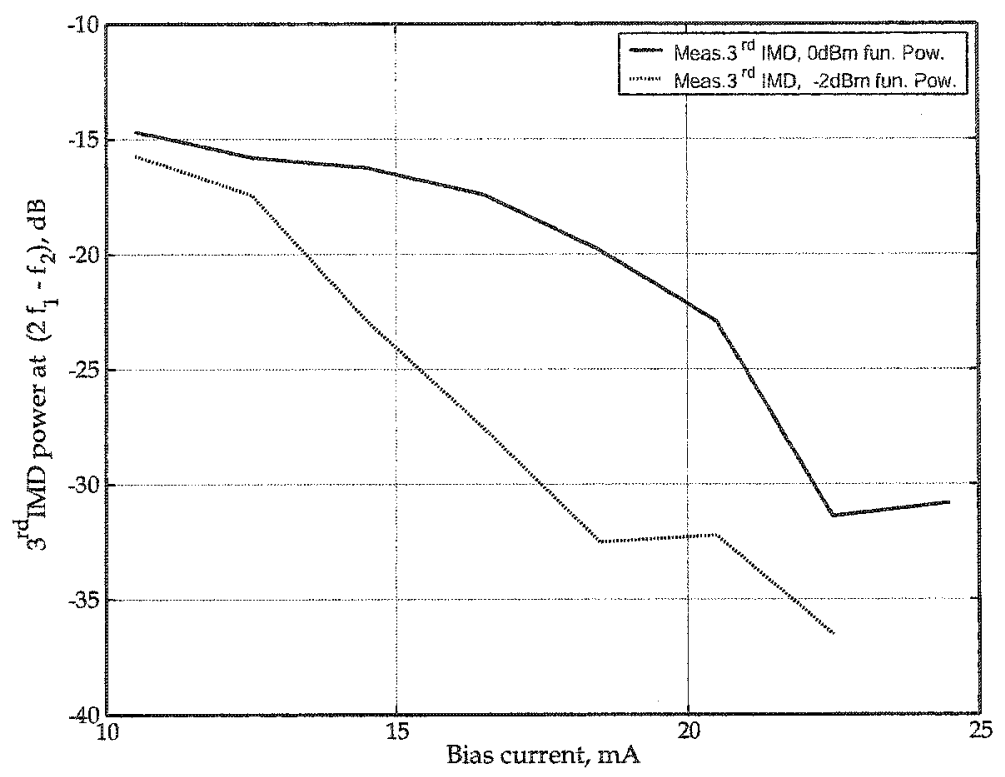

Figure 4.11: Measured lower $\left(2 f_{1}-f_{2}\right)$ third order intermodulation distortion as a function of the bias current. The carrier frequencies are $f_{1}=0.98$ and $f_{2}=1.02 \mathrm{GHz}$.

\subsection{Noise measurements}

Laser noise is an important limiting factor in determining the performance of optical systems. The relative intensity noise (RIN) and the frequency or phase noise are the main laser noise categories. Phase noise may cause a frequency shift which manifests as linewidth broadening. RIN and laser linewidth measurements are the focus of this section.

\subsubsection{RIN measurements}

Measurements of the RIN lead to the evaluation of the maximum achievable carrier-tonoise ratio $(\mathrm{C} / \mathrm{N})$ in the optical transmission system. The laser noise is the dominant noise source in short and medium length optical links where the fiber loss is low. The maximum achievable $(\mathrm{C} / \mathrm{N})$ depends on the carrier frequency, while the lowest $(\mathrm{C} / \mathrm{N})$ approximately around the relaxation oscillation frequency. Agilent $71400 \mathrm{C}$ lightwave signal analyzer with an RIN measurement module (special software routine) are used for the laser RIN measurements. The two main measurements that can be done using the RIN module 
are: (1) RIN SYS which is equivalent to the laser intensity noise, shot and thermal noise of the instrument. (2) $R I N_{\text {laser }}$ which is equivalent to the ratio of the laser noise to the average power without including the shot and thermal noise terms. $R I N_{S Y S}$ and $R I N_{\text {laser }}$ measurements are limited by the available optical power of the laser and the noise floor (shot and thermal) of the analyzer.

Actual RIN measurements of a $1.3 \mu m$ InGaAsP-InP (Fujitsu FLD3F7CZ) laser diode at four different bias levels $\left(10 \mathrm{~mA} \simeq 1.25 I_{t h}, 11.7 \mathrm{~mA} \simeq 1.5 I_{t h}, 15.2 \mathrm{~mA} \simeq 2 I_{t h}\right.$, and $\left.23.4 m A \simeq 3 I_{t h}\right)$ are shown in Figures 4.12-4.15.

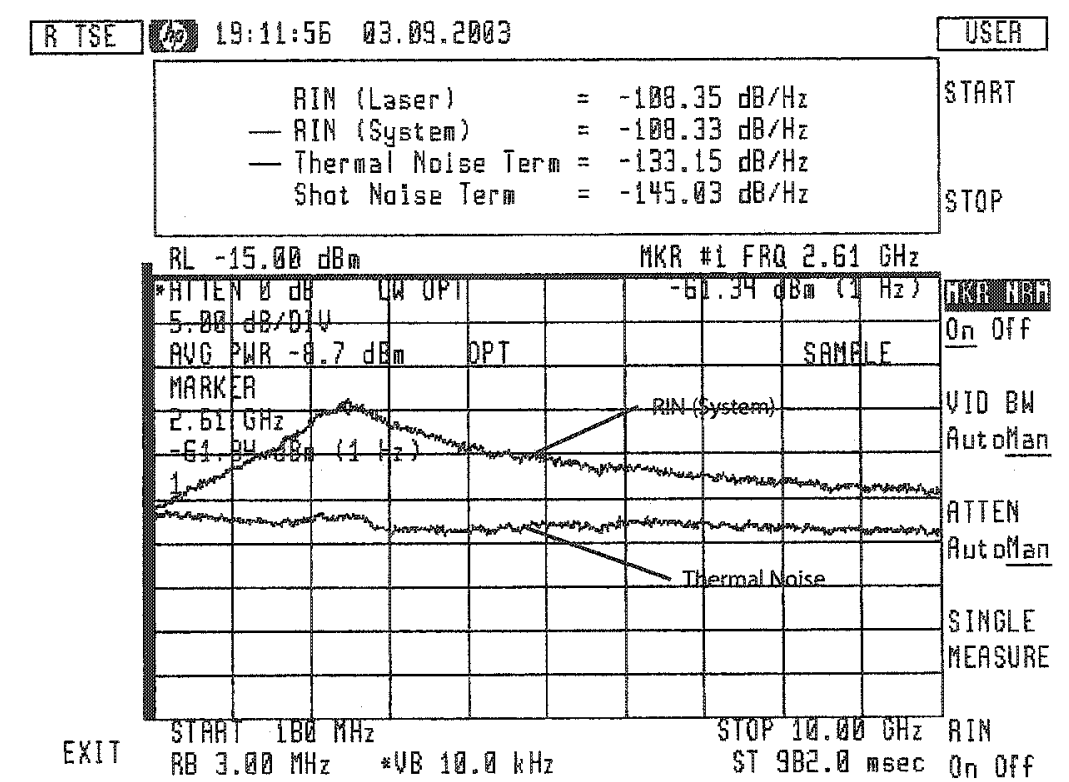

Figure 4.12: RIN measurement of FLD3F7CZ laser diode in the frequency range of $180 \mathrm{MHz}$ to $10 \mathrm{GHz}$ at $10 \mathrm{~mA}\left(\simeq 1.25 I_{t h}\right)$ bias level using Agilent $71400 \mathrm{C}$ lightwave signal analyzer. The data window on top shows the measured values at the marker frequency $(2.61 \mathrm{GHz})$.

Figures 4.12 to 4.15 show that the laser RIN is maximum near the relaxation oscillation frequency. The position of the RIN peak moves up on the frequency axis as the bias current increases. At lower bias levels, the RIN decreases faster as the bias level increases while it decreases slowly at higher bias levels. Figure 4.12 shows that $R I N_{\text {laser }}$ varies between -128.5 and $108.35 \mathrm{~dB} / \mathrm{Hz}$ in the frequency range from $350 \mathrm{MHz}$ to $10 \mathrm{GHz}$ which is critical 
in low-noise wideband applications and shows the importance of choosing the bias point that is suitable for the required application.

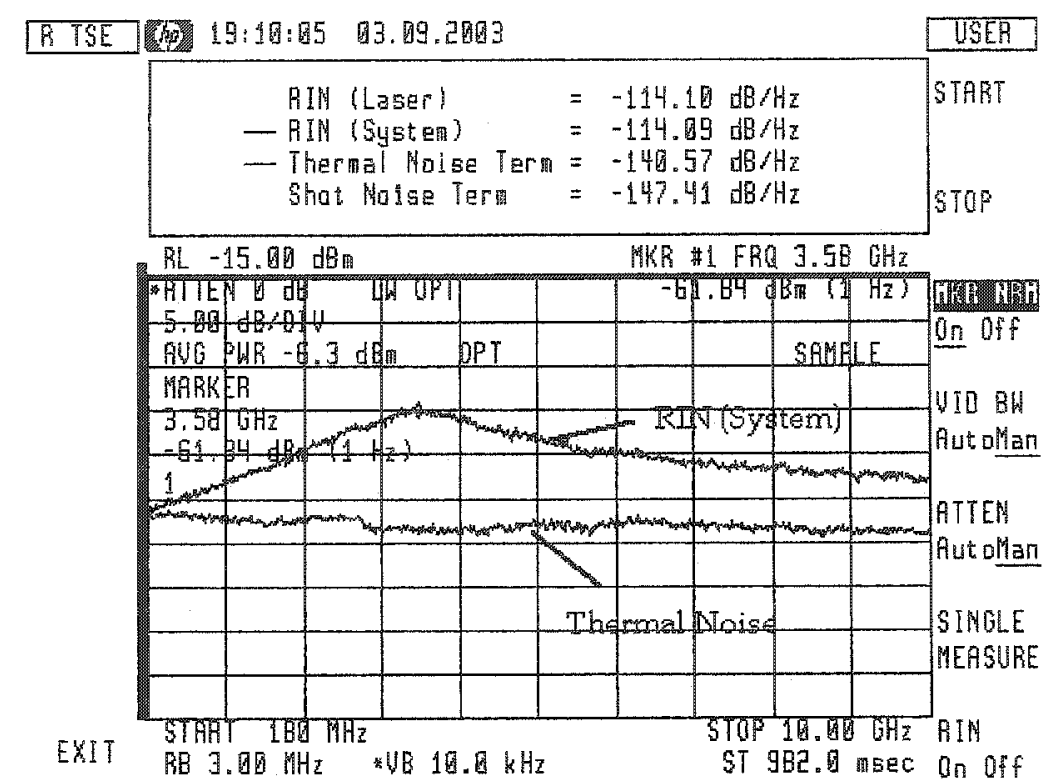

Figure 4.13: RIN measurement of FLD3F7CZ laser diode in the frequency range of $180 \mathrm{MHz}$ to $10 \mathrm{GHz}$ at $10 \mathrm{~mA}\left(\simeq 1.5 I_{t h}\right)$ bias level at $(3.58 \mathrm{GHz})$.

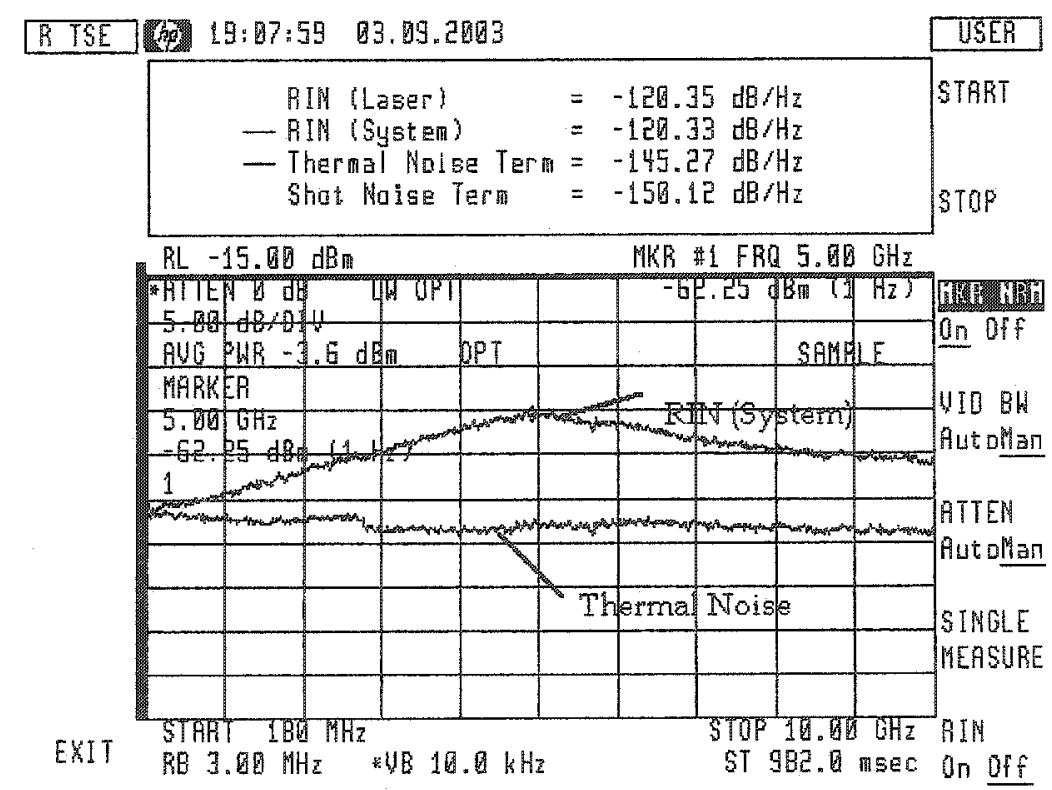

Figure 4.14: RIN measurement of FLD3F7CZ laser diode in the frequency range of $180 \mathrm{MHz}$ to $10 \mathrm{GHz}$ at $10 \mathrm{~mA}\left(\simeq 2 I_{t h}\right)$ bias level at $(5 \mathrm{GHz})$. 


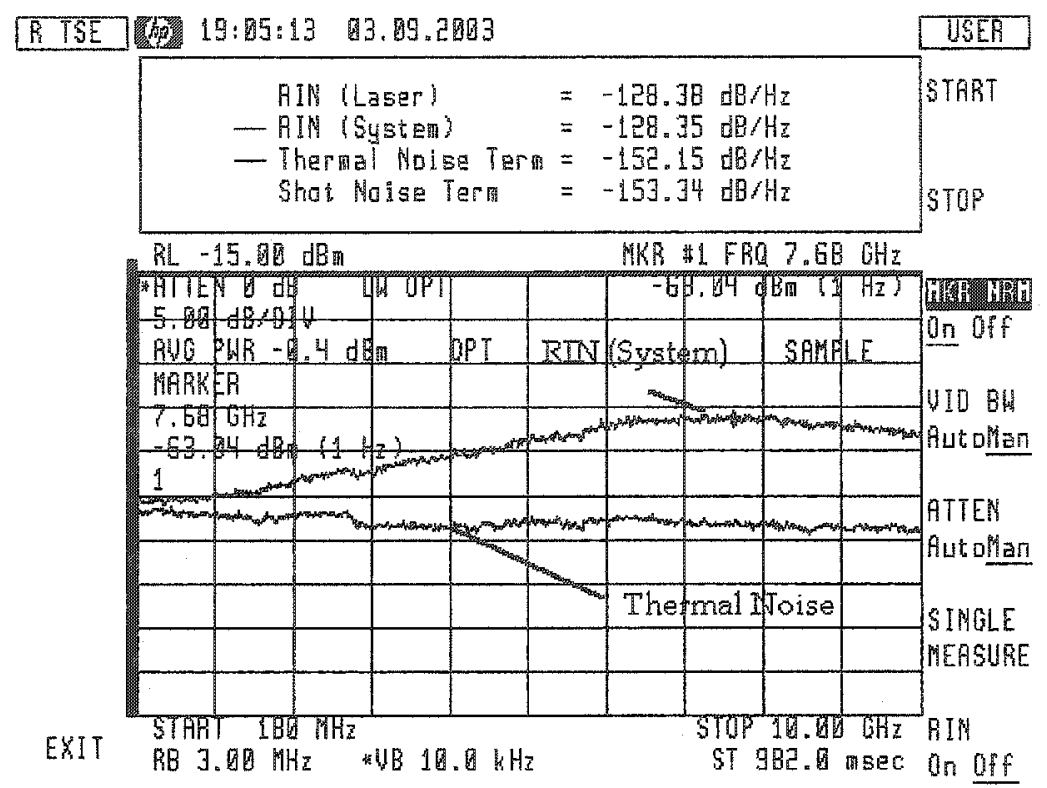

Figure 4.15: RIN measurement of FLD3F7CZ laser diode in the frequency range of $180 \mathrm{MHz}$ to $10 \mathrm{GHz}$ at $10 \mathrm{~mA}\left(\simeq 3 I_{t h}\right)$ bias level at $(7.68 \mathrm{GHz})$.

\subsubsection{Laser linewidth measurements}

Optical phase noise and frequency chirp have substantial impact on the optical power spectrum and the quality of transmission. Phase noise and frequency chirp are the main causes of the spectral broadening in a single-longitudinal-mode laser. Spectral broadening increases the error rates in the communication systems and also limits the proximity of channels in WDM systems [147]. Random phase noise and relaxation oscillations change the phase of the freerunning laser frequency. Laser frequency chirp is created due to the effect of the material and structural properties of the modulated laser causing linewidth broadening well beyond the free-running optical linewidth. Laser linewidth is the measure of the phase noise presented at the output of the laser diode. In this section, measurements of the linewidth of a free-running single mode laser (1.3um InGaAsP-InP Fujitsu FLD3F7CZ) were made using delayed self-homodyne technique. Agilent 71400C lightwave signal analyzer and Agilent 11980A interferometer with 70880A laser linewidth measure- 
ment software were used to characterize the linewidth. The photograph of the experimental setup shown in Figure 4.16 was used for the measurement of the linewidth. Linewidth measurements of a $1.3 \mu \mathrm{m}$ InGaAsP-InP (Fujitsu FLD3F7CZ) laser diode at three different bias levels $15.2,23.4$, and $60 \mathrm{~mA}$ are shown in Figures 4.17 - 4.19 .

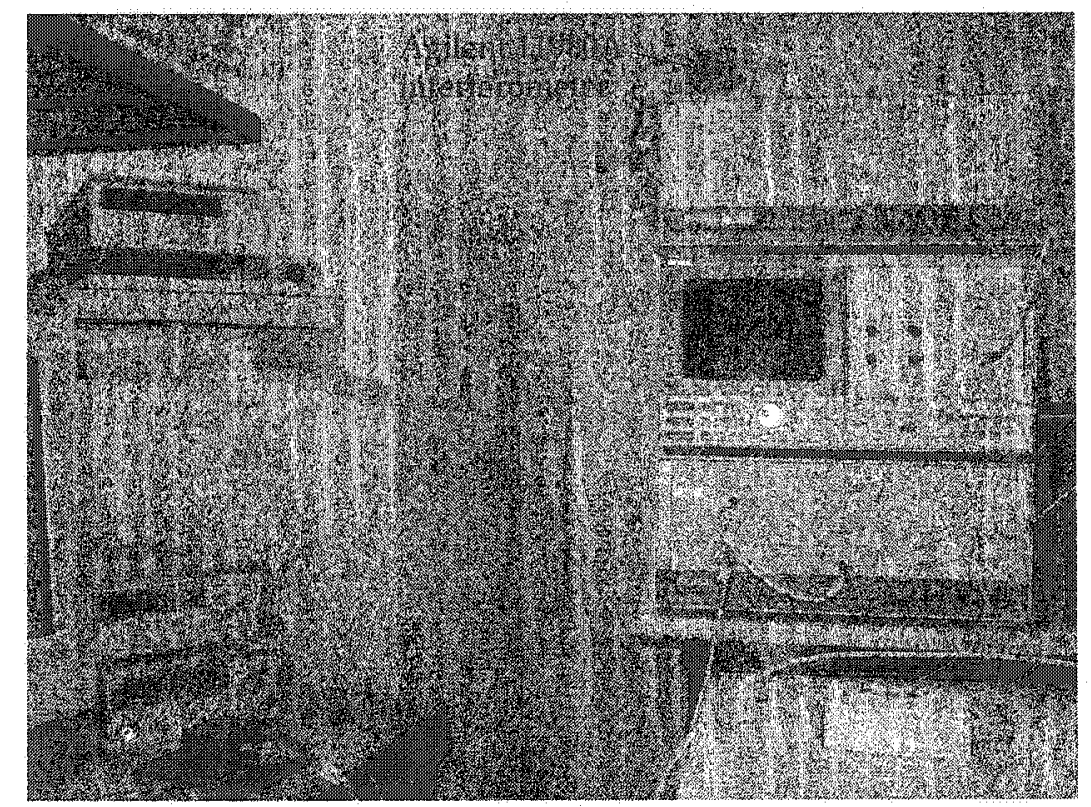

Figure 4.16: Experimental setup for laser linewidth measurements.

The measured linewidth shown in the top window in Figures $4.17-4.19$ is the actual 3$\mathrm{dB}$ linewidth of the laser. From these figures, the linewidth decreases as the bias level increases. The Lorentzian fit shown in these figures is calculated from the ideal Lorentzian line shape. The Lorentzian fit standard deviation is obtained by calculating the linewidth from each trace point and for 800 trace points. The root-mean square deviation of the individual linewidth from the least square fit linewidth results in the standard deviation measurement of the linewidth as shown in the top window of each figure. As shown in Figures 4.17 - 4.19 the trace data and the calculated distribution curve overlap on the display which means that the laser line shape at different bias levels is Lorentzian and the standard deviation in this case is a measure of the average trace noise. 


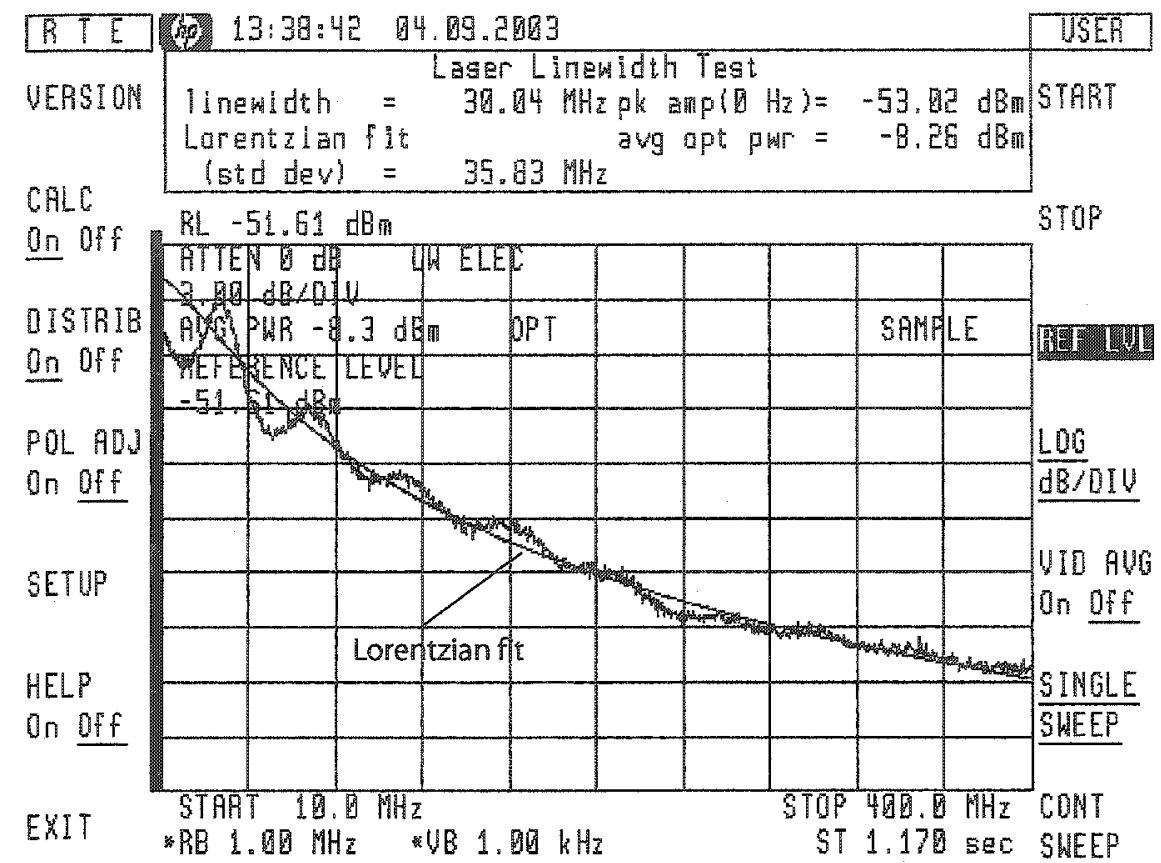

Figure 4.17: Laser linewidth measurement of FLD3F7CZ laser diode at $15.3 \mathrm{~mA}\left(\simeq 2 I_{t h}\right)$ bias level using Agilent $71400 \mathrm{C}$ lightwave signal analyzer. The data window on top shows the measured values.

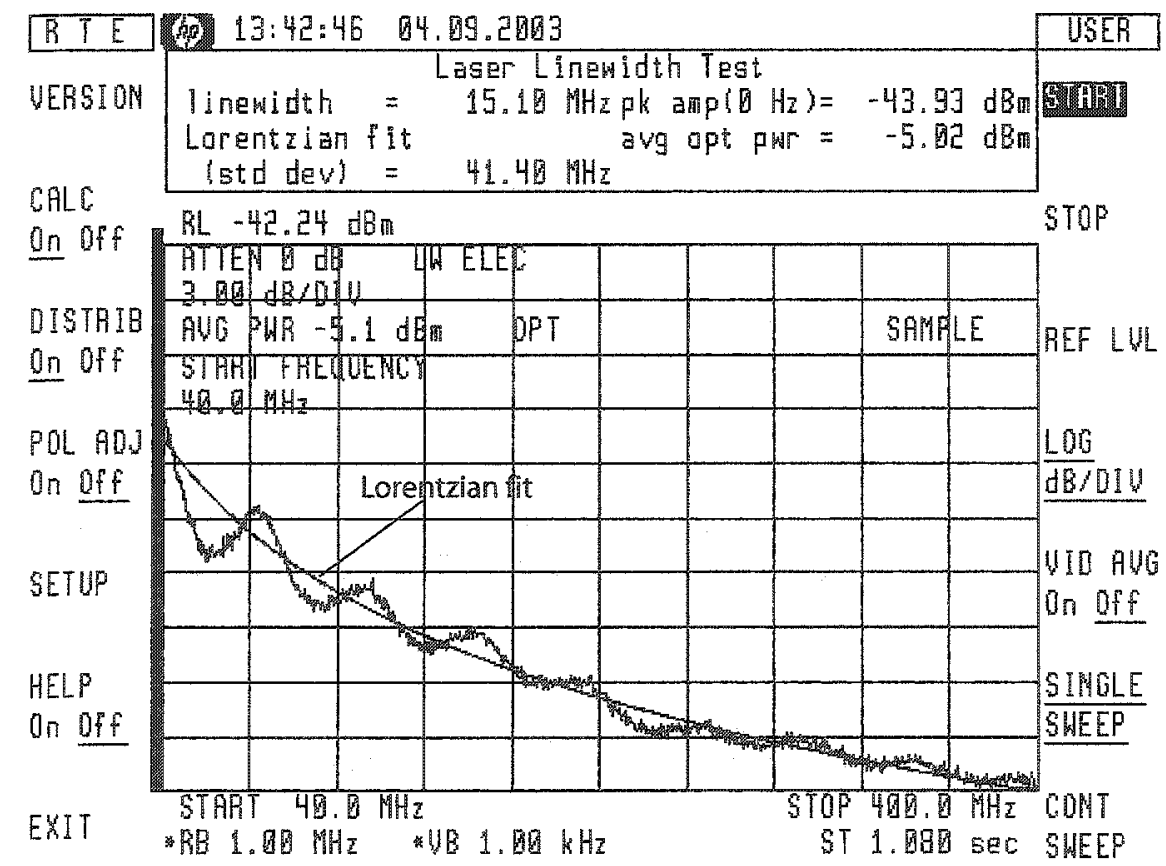

Figure 4.18: Laser linewidth measurement of FLD3F7CZ laser diode at $23.4 \mathrm{~mA}\left(\simeq 3 \mathrm{I}_{\mathrm{th}}\right)$ bias level using Agilent $71400 \mathrm{C}$ lightwave signal analyzer. 


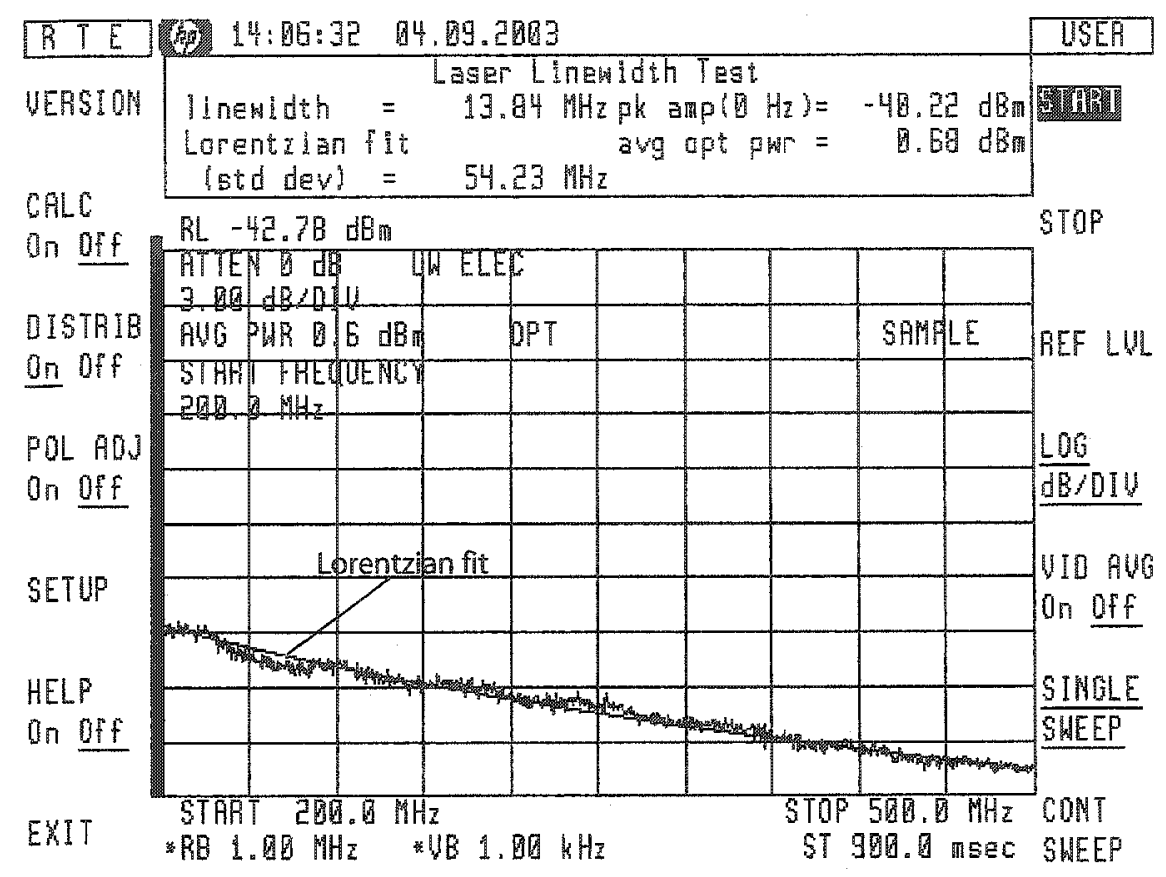

Figure 4.19: Laser linewidth measurement of FLD3F7CZ laser diode at $60 \mathrm{~mA}$ bias level using Agilent $71400 \mathrm{C}$ lightwave signal analyzer.

\subsection{Model verification}

To verify the proposed laser models, we compared the DC, modulation frequency response, and nonlinearity measurements with the corresponding simulation results under various conditions. For the purpose of this comparison, parameters suitable for the laser under test (InGaAsP-InP Fujitsu FLD3F7CZ) are extracted using the parameters extraction algorithm presented in Section G.4 of Appendix G. Figure 4.20 illustrates the measured and simulated results of the DC light versus bias current. From Figure 4.20 , the simulation results using the proposed model based on the extracted parameters stated in Section G.4 of Appendix $G$ are in good agreement with the measured results. The DC comparison is important in the determination of the threshold current which is one of the key parameters for the system level simulations.

In Figures $4.21-4.23$, the frequency response for Fujitsu FLD3F7CZ laser was measured 


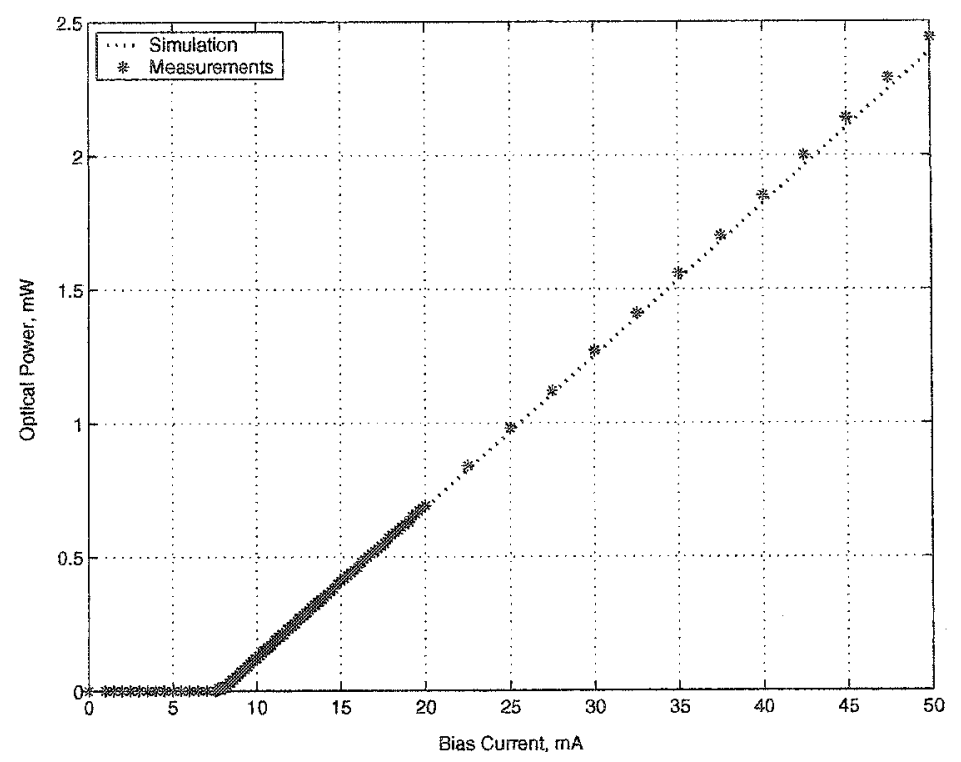

Figure 4.20: Simulation and measurement results of the output optical power versus bias current characteristics at $27 C$ for a $1.3 \mu \mathrm{m}$ InGaAsP-InP Fujitsu FLD3F7CZ butterfly packaged semiconductor laser.

at various bias levels and compared with the simulation results using the proposed model. As shown in Figures $4.21-4.23$ the resonance frequency gradually subsides as the bias current (optical power) increases which being flat for the overall response. This means that the peak of the relaxation frequency decreases as the bias current increases, which a.grees with the simulation results using the proposed model. From these measurements, we deduce that the modulation bandwidth is an increasing function of the bias current which is consistent with the simulation results using the proposed model as discussed in Chapter 3. Many important parameters for both systems and device designers that have significant impact on the performance of the whole optical communication system can be predicted from these plots. Examples of such parameters include the relaxation oscillation frequency, modulation bandwidth.

From Figures $4.21-4.23$, we conclude that the simulation results are in good agreement with the measured results, specially in the frequency range up to $3 \mathrm{GHz}$ which is suitable 


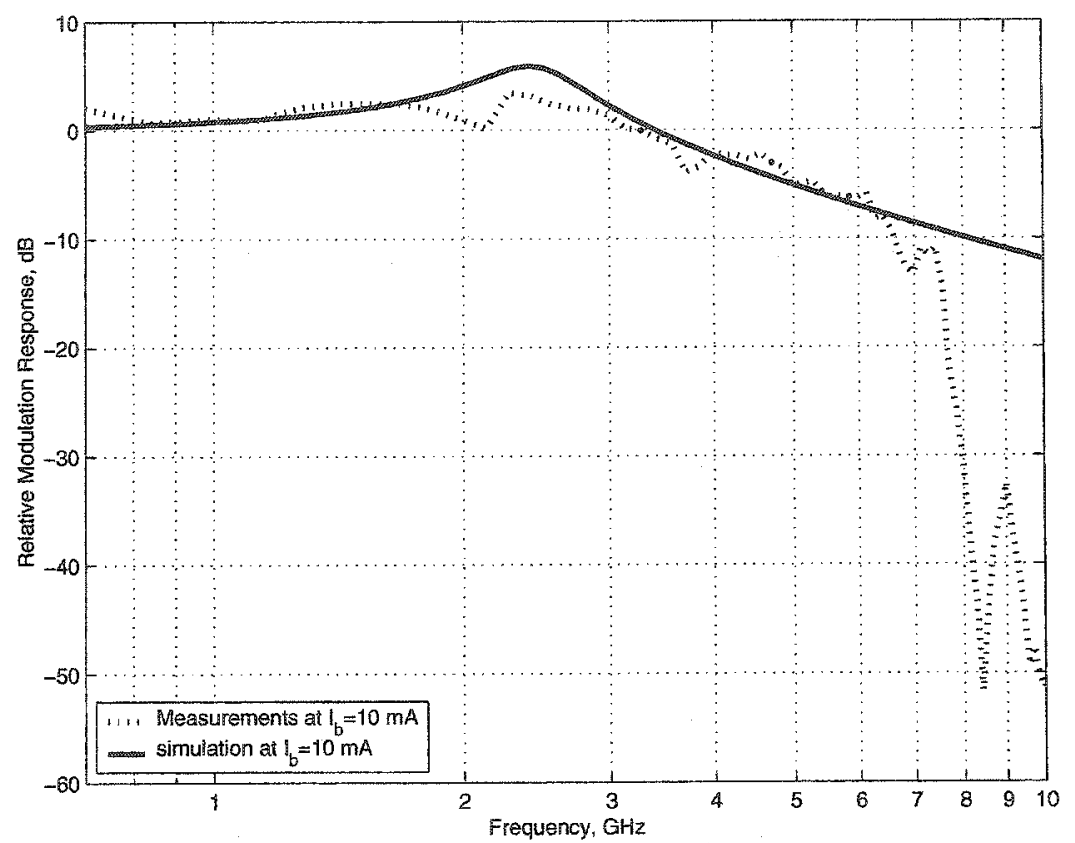

Figure 4.21: Simulation and measurement results of the modulation frequency response for Fujitsu FLD3F7CZ laser at $10 \mathrm{~mA}$ bias levels .

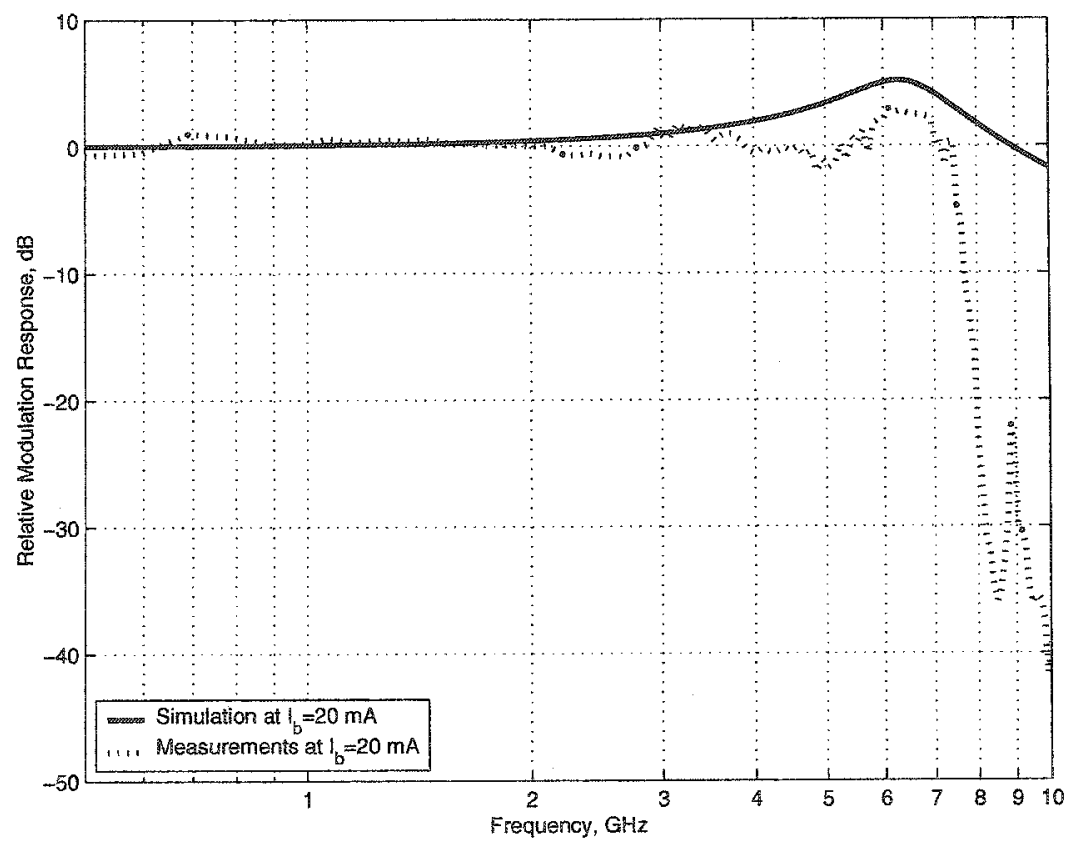

Figure 4.22: Simulation and measurement results of the modulation frequency response for Fujitsu FLD3F7CZ laser at $20 \mathrm{~mA}$ bias levels [9]. 


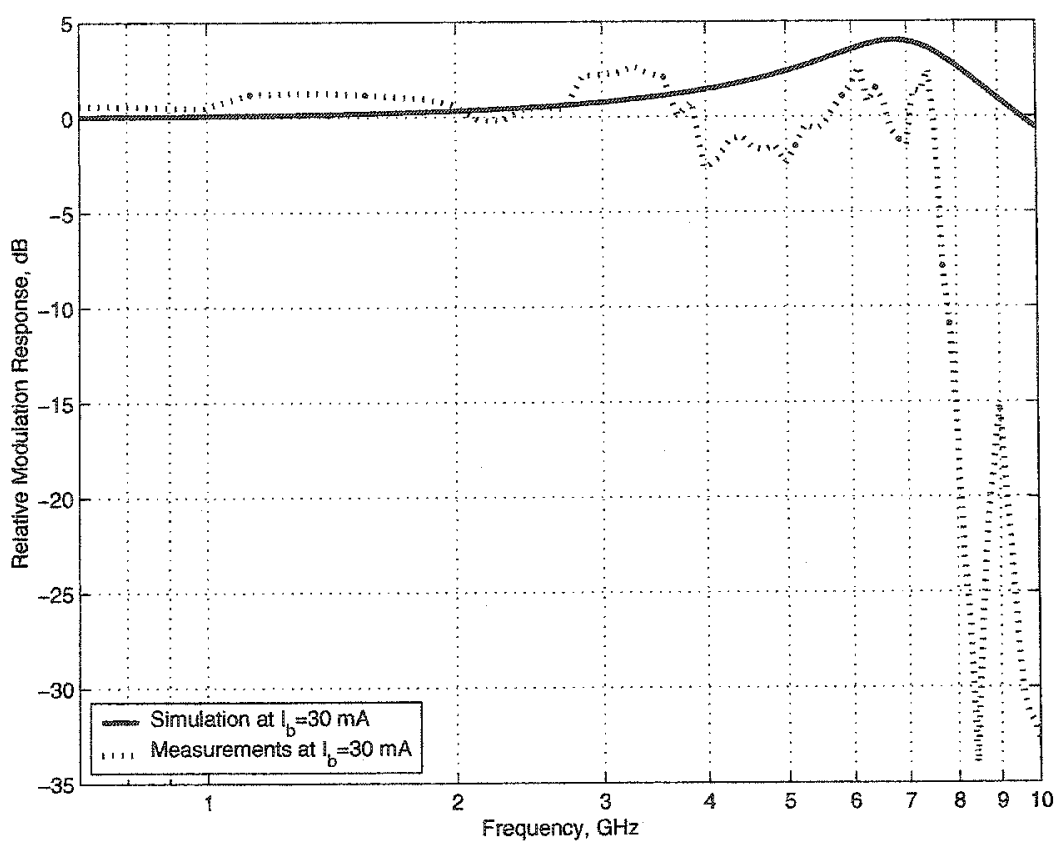

Figure 4.23: Simulation and measurement results of the modulation frequency response for Fujitsu FLD3F7CZ laser at $30 \mathrm{~mA}$ bias levels.

for the intended application. The discrepancies between the measured and the simulated results in Figures 4.21 - 4.23 can be attributed to the fact that the model does not take into account the matching circuit of the laser mount and the electrical parasitics of the laser package.

Transmitter linearity is an important consideration in the design of wideband transmitters for analog lightwave transmission systems since it limits the dynamic range of the whole transmission link [9]. Since the dynamic range of the directly modulated lasers are mainly limited by the distortion levels, accurate simulations for the nonlinear distortion are important for improving the laser diode linearity. This improvement leads in turn to significant improvement of the performance of the whole system.

For further validation of the model, measurements of the second harmonic distortion (2HD), second and third intermodulation distortions (2IMD and 3IMD) are compared with the 
simulation results of the same distortion components. These results are illustrated in Figures $4.24-4.28$.

We selected these distortion components because the superior free dynamic range of the optical link is measured as the difference between the power of one of the fundamental tones and the power of the highest distortion components that fall in the bandwidth of interest above the noise floor. For wideband applications, second harmonic and second intermodulation distortion are significant. While for the applications operating within one octave, the third order intermodulation distortion is significant.

The Simulation and experimental study in this part of the comparison consists of direct modulation of the laser with two tones that are $40 \mathrm{MHz}$ apart and monitoring a variety of sum and difference generated frequencies. Figures 4.24 and 4.25 illustrate the comparison between the measurement and simulation results of the second harmonic distortion for two different carrier powers. The comparison of the simulated second order intermodulation distortion with the measured 2IMD is shown in Figure 4.26. The measured and simulated lower $\left(2 f_{1}-f_{2}\right)$ and higher $\left(2 f_{2}-f_{1}\right)$ components of the third order intermodulation distortion at different bias levels are illustrated in Figures 4.27 and 4.28 respectively. The measurements and simulations were done using two tone of frequencies $f_{1}=0.98$ and $f_{2}=1.02 \mathrm{GHz}$ and repeated for signal powers 0 and $-2 \mathrm{dBm}$. The major observed features predicted by the proposed model and verified by the experimental results as shown in Figures $4.24-4.28$ are:

- Distortion is a decreasing function of the bias current up to saturation.

- The relative amplitude of the distortion components is an increasing function of the input signal power and frequency.

- Measurements at specific bias or input power or frequency are not a reliable indication for the device performance under different conditions. 
The results Illustrated in Figures $4.20-4.28$ confirmed that the predicted performance using the proposed model is in good agreement with the measured performance. This means that the proposed models are robust and can be trusted in predicting the device performance under different conditions and for different applications.

Including the matching circuit and package model of the laser will enhance the model ability in predicting the modulation frequency response. Also, including the effect of reflected waves from the laser mirrors and the fiber pigtail may reduce the discrepancies between experimental and simulation results in Figures 4.24 to 4.28 . However, these differences are not significant when taking into account the ability of the proposed models to predict different characteristics under different conditions and for different applications.

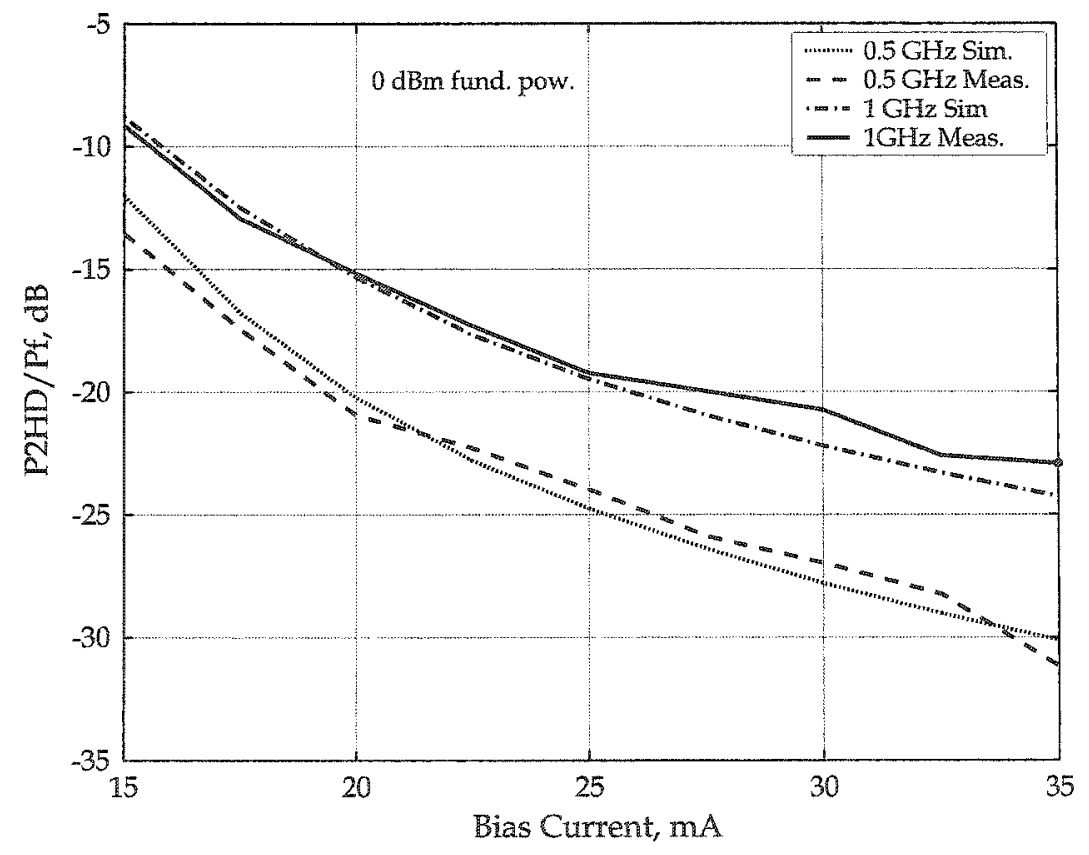

Figure 4.24: Simulation and measurement of the second harmonic distortion as a function of the bias current at carrier frequencies 0.5 and $1 \mathrm{GHz}$ and power $0 \mathrm{dBm}$ [10]. 


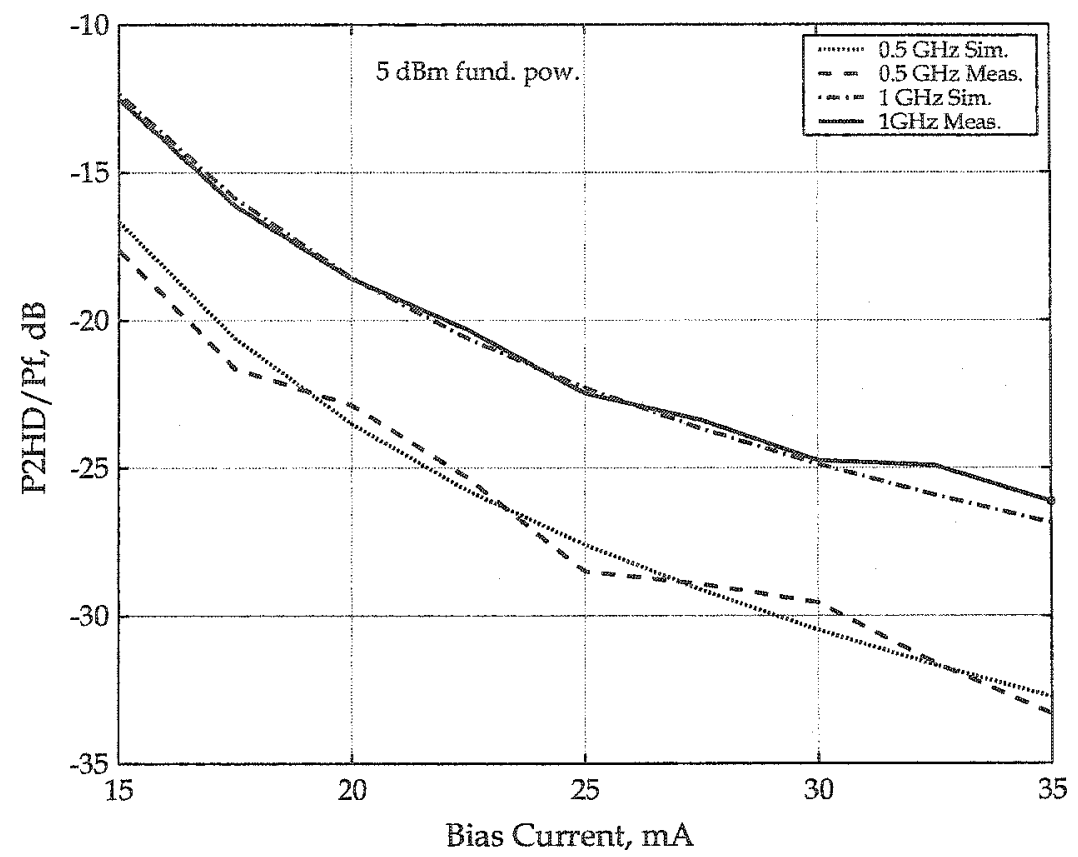

Figure 4.25: Simulation and measurement of the second harmonic distortion as a function of the bias current at carrier frequencies 0.5 and $1 \mathrm{GHz}$ and power $-5 \mathrm{dBm}$.

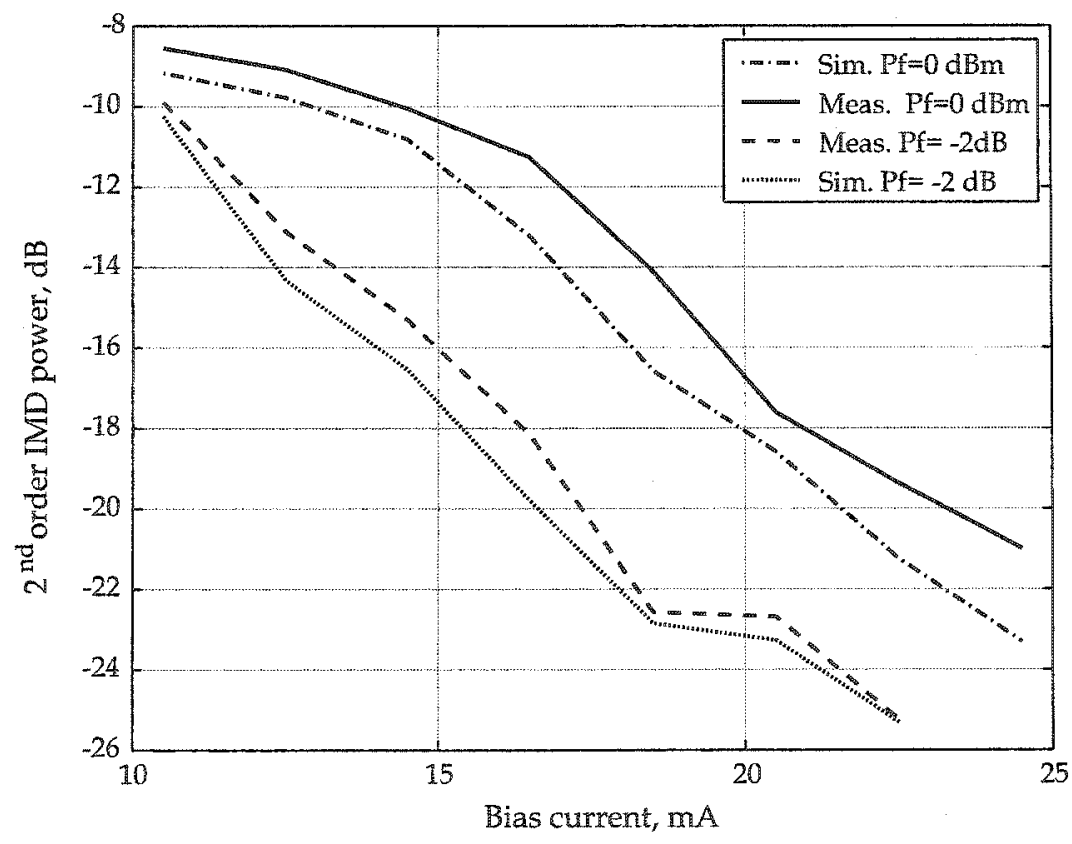

Figure 4.26: Measured and simulated second order intermodulation $\left(f_{1}+f_{2}\right)$ distortion power as a function of the bias current at carrier frequencies $f_{1}=0.98$ and $f_{2}=1.02$ $\mathrm{GHz}$. 


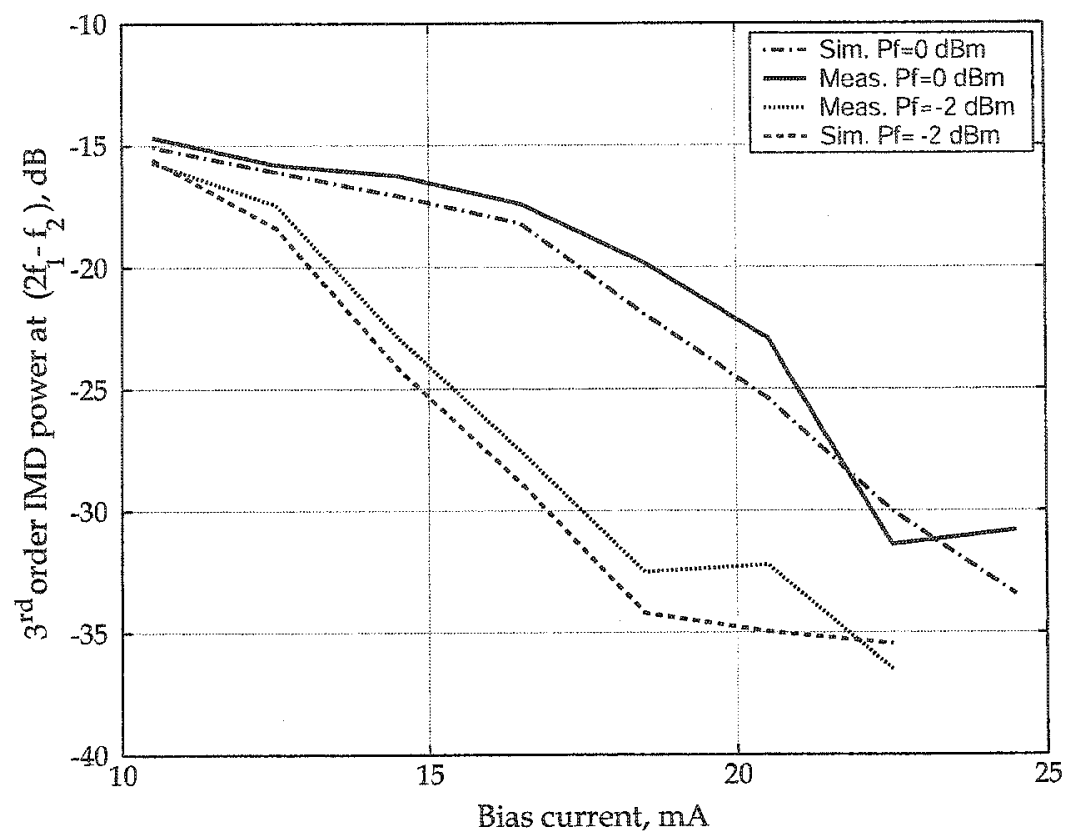

Figure 4.27: Measured and simulated lower $\left(2 f_{1}-f_{2}\right)$ third order intermodulation distortion components as a function of the bias current at carrier frequencies $f_{1}=0.98$ and $f_{2}=1.02 \mathrm{GHz}$.

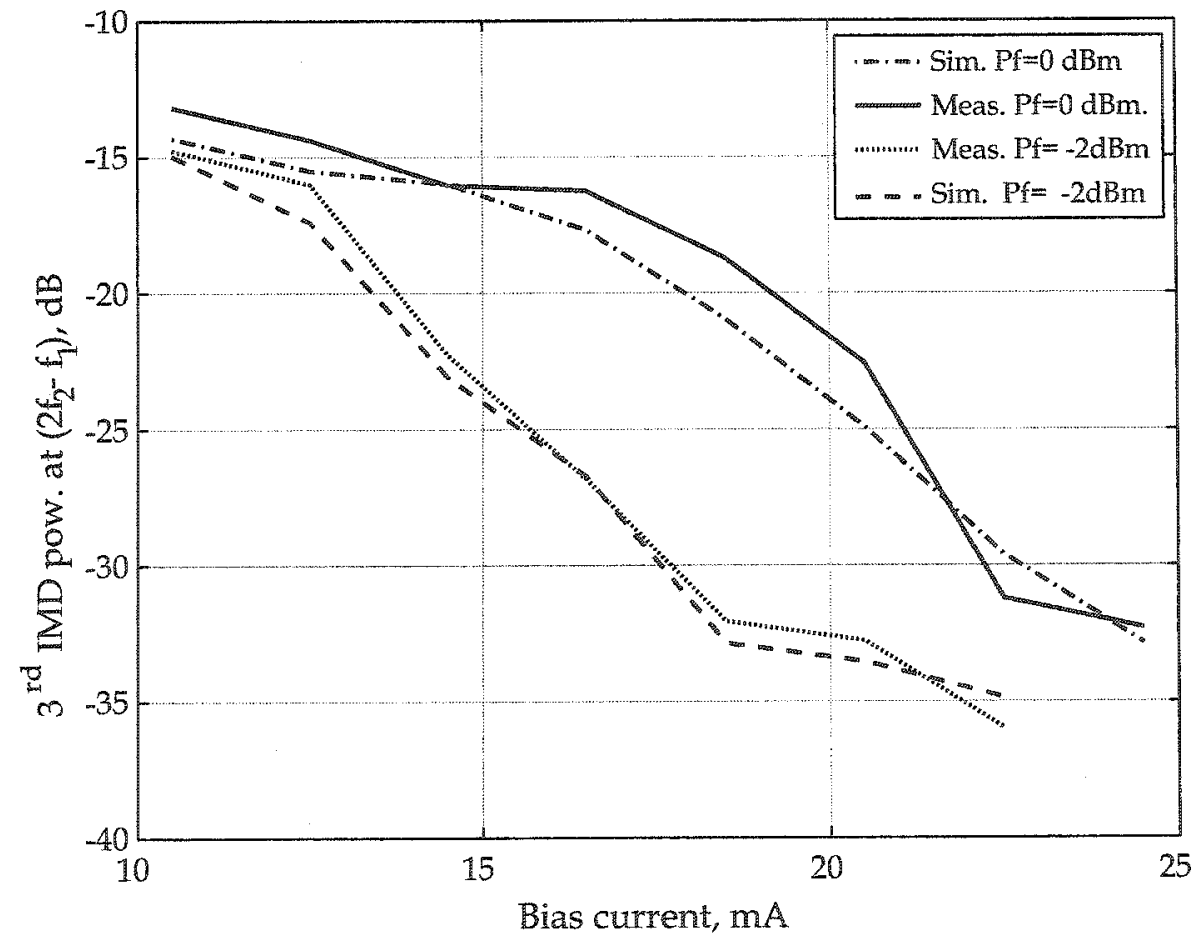

Figure 4.28: Measured and simulated higher $\left(2 f_{2}-f_{1}\right)$ third order intermodulation distortion components as a function of the bias current at carrier frequencies $f_{1}=0.98$ and $f_{2}=1.02 \mathrm{GHz}$. 


\section{Chapter 5}

\section{Nonlinearity and Linearization Techniques}

\subsection{Concepts of nonlinearity}

Nonlinear circuits are often characterized as either strongly nonlinear or weakly nonlinear.

Weakly nonlinear circuits can be described with adequate accuracy using time domain techniques such as power series expansion, and can be analyzed by power series or Volterra series. Strong nonlinear circuits can be analyzed using frequency-domain techniques. Two common frequency domain techniques are harmonic-balance and Volterra-series or nonlinear transfer function analysis [148].

\subsubsection{Harmonic generation}

Nonlinear system generate harmonics of the excitation signal frequency, and cause what is called harmonic distortion, HD. Harmonics may interact with one or more of the excitation frequencies or with each other creating cross product terms given as $n \omega_{1}+m \omega_{2}$, where $\mathrm{m}$ and $\mathrm{n}=0, \pm 1, \pm 2, \pm 3, \ldots$ This phenomenon is called intermodulation distortion. The sum of the absolute values of the coefficients $m$ and $n$ determines the order of the intermodulation distortion (MD). For example, second order intermodulation products (2MMP) are at $\left|\omega_{1} \pm \omega_{2}\right|$, the third order intermodulation products are at $\left|\omega_{1} \pm 2 \omega_{2}\right|$ and $\left|2 \omega_{1} \pm \omega_{2}\right|$, and so on. Intermodulation products often present a serious problem, because they represent signals that interfere with, and can be mistaken for, desired signals. In general, the odd order intermodulation products having $m=n \pm 1$ are the source of most 
of the problems because they may fall within the desired bandwidth. Third and fifth order terms are important because they are typically the strongest of all odd-order products, close to the generating signals, and seldom rejected by filters. Second order terms are also problematic in broadband applications, and at low frequencies.

\subsubsection{Nonlinearity in semiconductor lasers}

Using Radio-Over-Fibre (ROF) links in microcellular wireless networks enables increased signal processing at the central station. For the purpose of this research, directly modulated ROF links are considered. A primary concern when a wireless link is in series with an optical link in a multi-user environment is the nonlinear distortion (NLD) of the ROF link. An inhibiting factor for analog wireless/optical networks is the natural nonlinearity of semiconductor lasers which degrades the overall system performance and capabilities [149]. The dominant nonlinearity at higher frequencies appears when the input signal frequency becomes comparable to the resonant frequency of the optical cavity. This is known as resonance distortion and it is a result of the nonlinear coupling between the photons and the injected electrons $[3,6,82,83,150]$. There are many other mechanisms that highly affect the linearity of the laser diodes such as spatial hole burning $[6,106]$, carrier heating $[66,100]$, current leakage $[7,33,107]$, intervalence band absorption [117] and free-carrier absorption [33]. Furthermore, optical reflections or feedback may become a source of nonlinearity within the laser or may interfere with the fibre chirp causing frequency modulation (FM) $[22,81,151-153]$.

\subsection{Review of linearization techniques}

Transmitter linearity is an important consideration in the design of wideband transmitters for analog lightwave transmission systems, since it limits the dynamic range of the 
whole link. The transmission quality of optical transmitters can be described by carrierto-noise ratio (CNR), composite triple beat (CTB), and composite second order (CSO) distortion $[11,24,25,154-156]$. The CSO and CTB are the ratios of the carrier to the total power within the largest accumulation of even and odd order distortion products at the carrier frequency. Published results indicate that in a typical microcellular environment with obstructions, $80-90 \mathrm{~dB}$ dynamic range (DR) is required in the uplink, whereas larger DR is required in the down link $[149,157]$. CATV applications generally impose limitations on allowable nonlinearities in the transmitter [158] and require a carrier to noise ratio $(\mathrm{CNR})>50 \mathrm{~dB}[25,159]$. The highest published spurious free dynamic range (SFDR) in a directly modulated $\mathrm{ROF}$ links was $90-110 \mathrm{~dB} \mathrm{H} z^{\frac{2}{3}}$ using distributed feedback (DFB) lasers, and $70-90 \mathrm{~dB} \mathrm{~Hz} z^{\frac{2}{3}}$ using Fabry-Perot (FP) lasers [160]. The results for the F.P. are less than required, due to the nonlinearity of the F.P. laser diodes. Improved fabrication techniques and device structures have lead to many successful applications, but fabricating linear lasers is an expensive task. Consequently, the possibility of linearization of inexpensive lasers (with characteristically poor linearity) by using circuit techniques to compensate for the inherent nonlinearity of the optical transmitter to the point where they perform as well as expensive lasers is an attractive alternative. A variety of linearization methods for semiconductor laser diodes suitable for specific applications can be classified as: optoelectronic feedback and feed-forward methods [11, 24-26, 159, 161], post-distortion [162-164], pre-distortion [165-171], and adaptive pre-distortion $[149,153,157,172]$. Techniques using quasi-feed-forward, phase-shift modulation, external injection, electro-absorption, dual parallel and cascaded modulation $[12,13,34,173-175]$ are also used, but they don't account for laser heating and aging. An alternative solution that accounts for the effects of laser aging and heating and suitable for high frequency and broadband applications would be valuable for use in analog optical links. The ideal solution would be highly integrated, perhaps in the form of a single-chip, for reasons of economy. 


\subsubsection{Optoelectronic feedback}

Optoelectronic feedback is based on the concept of negative feedback for linearization. In this method a monitoring photodiode and a feedback network are used to provide negative electrical feedback. Figure 5.1 shows a simplified block diagram of an optoelectronic feedback system. In this technique a photodiode is used to monitor the laser output.

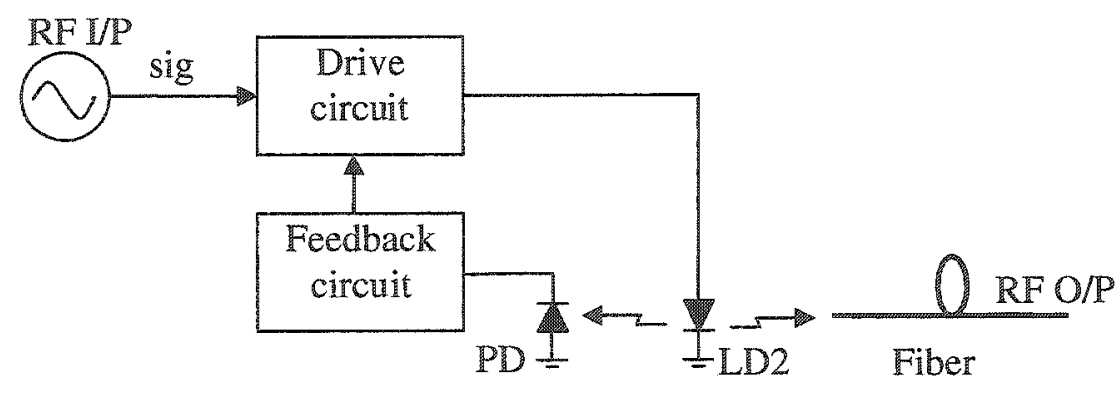

Figure 5.1: Optoelectronic feedback system.

Several experiments were carried out in the period from 1970's to 1980's based on the feedback technique. For the typical results using this technique, see Table 5.1 in Section 5.3. Negative feedback reduces both the second and third harmonic distortion, but it is not recommended at high frequencies, since the overall forward gain is reduced. At high frequencies loop stability becomes problematic, and tight requirements on the loop gain and bandwidth must be met. The enhancement levels expected from this linearization technique are limited by the matching between the gain and phase of the signal in the forward path and the feedback signal from the feedback paths. As a result, optoelectronic feedback is useful in narrowband applications, but it is not suitable for wideband applications.

\subsubsection{Optical feed-forward}

This technique uses the feed-forward method in order to linearize the output of the laser diode. The block diagram of the feedforward method is shown in Figure 5.2 [11,24-26]. As shown in Figure 5.2, the RF input signal $I$ is split into two paths. The first path contains 


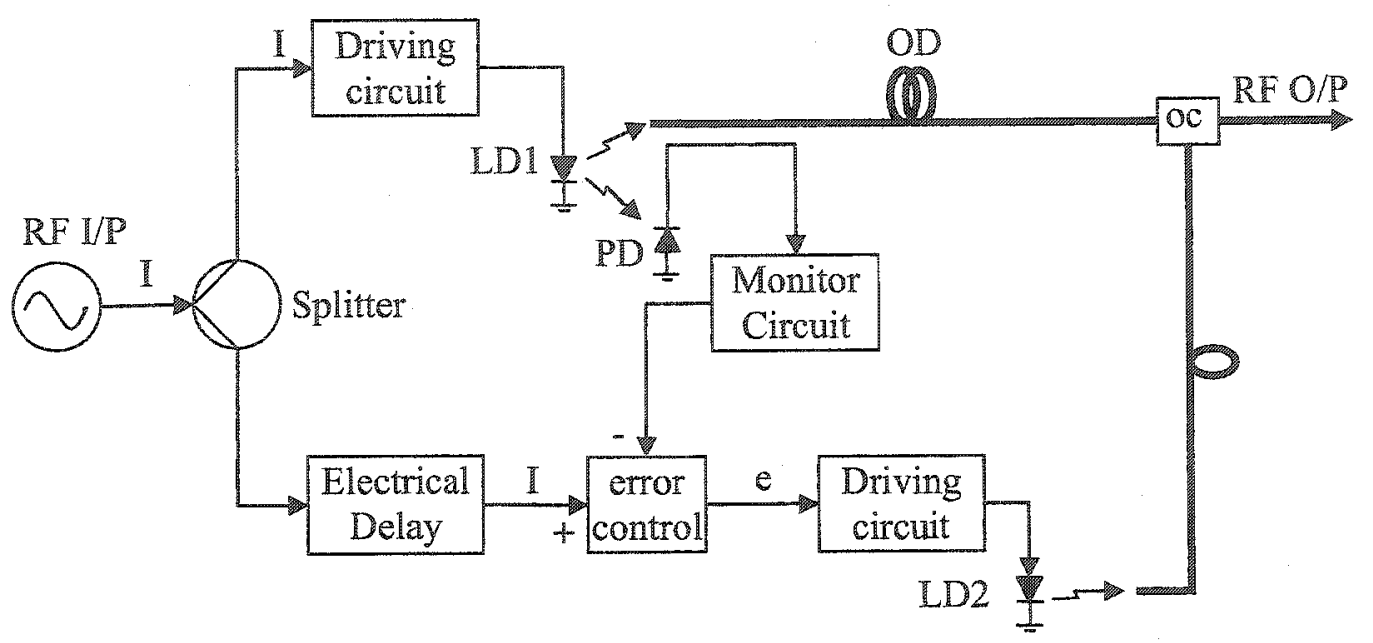

Figure 5.2: Optical feed-forward system [11].

the laser diode. The nonlinearly amplified optical signal is then split into two branches, one being delayed using an optical delay (OD) and the other monitored using a photodiode and subtracted from a delayed version of the RF input signal. Since the RF input contains only the desired signal while the photodiode output contains the desired signal and other harmonics due to the nonlinear nature of the laser diode, ideally the difference contains only the unwanted harmonics. This error signal is amplified through the second laser, which is then subtracted from the delayed version of the amplified RF signal of LD1 through an optical coupler (OC). Ideally, this technique cancels the nonlinearities of LD1, and the final output is a linear representation of the input. The feed-forward technique is completely open-loop and therefore avoids feedback instability issues. The main concern with this method is the matching between the two paths. The feed-forward technique reintroduces another LD to amplify the error signal. The additional LD should be as linear as possible in order to adequately cancel the error components in the output. Note that the method uses an optical power combiner, which may add more distortion to the signal. Cost and matching problems are the main drawbacks of this technique. Even with these drawbacks, the feed-forward technique can be valuable. The feed-forward technique can reduce laser 
noise as well as increase linearity. For the typical results using this technique, see Table 5.1 in Section 5.3. The above mentioned significant results make this technique very useful to linearize optical transmitters used in wideband applications but cost and high matching accuracy make it critical.

\subsubsection{Phase shift modulation}

In this technique, an additional laser diode is required to cancel a specific harmonic according to the amount of the phase shift between the two signals. $\mathrm{n}^{\text {th }}$ order distortion products can be cancelled when the phase angle between the two modulating signals is equal to $180^{\circ} / n\left(\phi_{n}=180^{\circ} / n\right)$. For the phase difference $\phi$ equal to $90^{\circ}$ the second order distortion can be eliminated. To eliminate the third order distortion the signal phase shift between the pairs should be $60^{\circ}$. Simultaneous cancellation of both second and third order distortion can be achieved by using two pairs of matched laser diodes as shown in Figure 5.3 [150]. This configuration can be repeated for higher order distortion cancellation. This technique

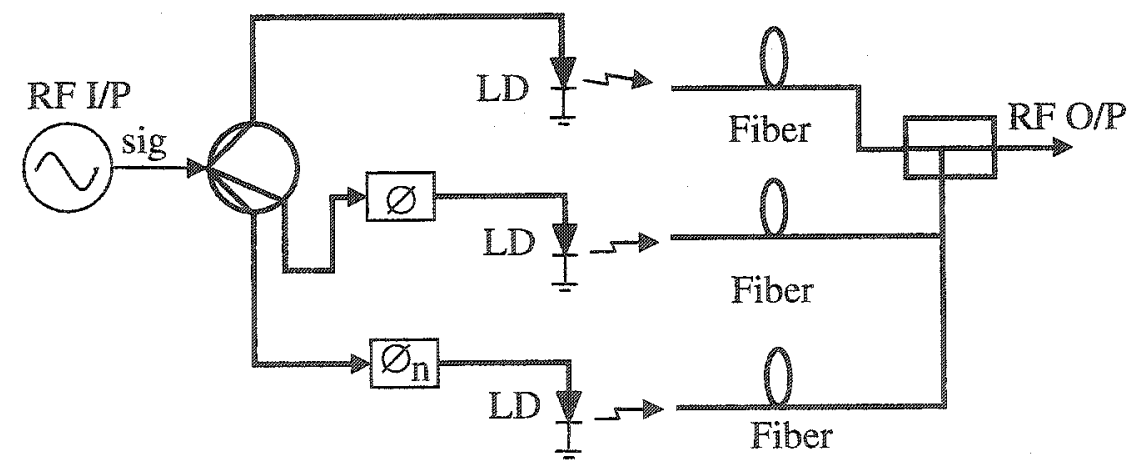

Figure 5.3: Phase shift modulation block diagram.

is not costly effective because an additional $\mathrm{LD}$ is required for each cancellation order. Also, matching between these LDs is required for high performance. 


\subsubsection{External light injection}

In this technique, shown in Figure 5.4, an external laser source called the master laser (usually a tunable laser source) is used in conjunction with a directly modulated source called the slave laser. The first experimental demonstration of analog optical fibre communication system employing a directly modulated semiconductor laser under optical injection locking was reported in $[12,173]$. For the typical results using this technique, see Table 5.1 in Section 5.3. G. Yabre and J. L. Bihan [34] introduced a theoretical investigation of the second and third order intermodulation distortions in an injection-locked semiconductor laser under small-signal modulation. Their results show that a substantial reduction of the laser nonlinearity can be obtained, depending on both the injection level and frequency detuning between the master and slave lasers.

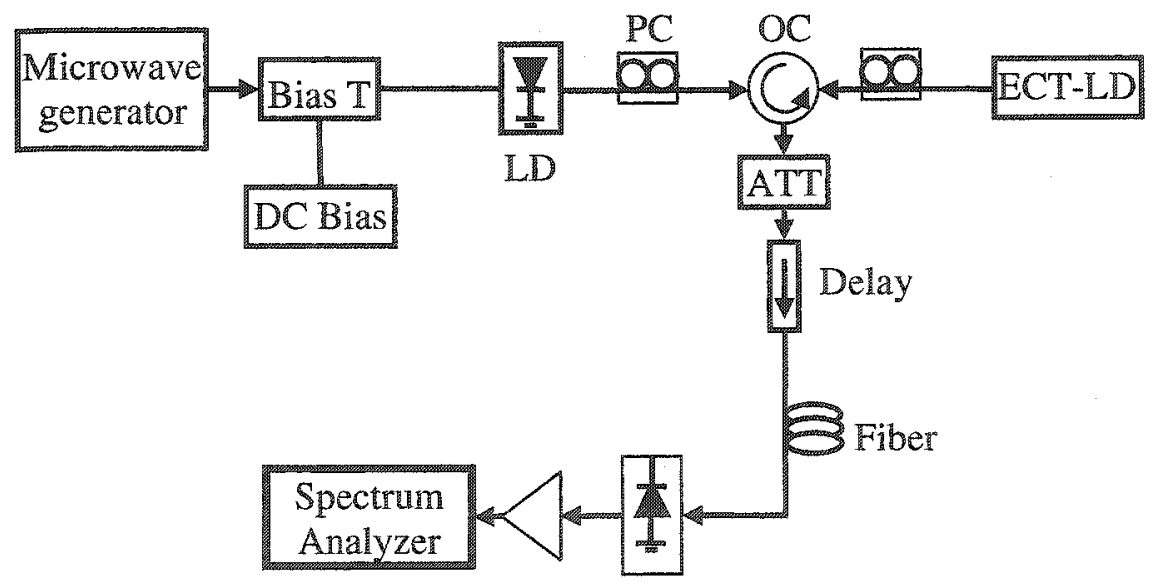

Figure 5.4: Schematic diagram of the analog fibre-optic system employing a directly modulated semiconductor laser with external light injection. OC: refer to optical circulator, PC: polarization control, ECT-LD: external cavity tunable laser diode, and ATT: attenuator [12]

\subsubsection{Dual-parallel modulation}

Using this technique, two laser diodes operating at different wavelengths and an optical combiner are used. Intermodulation distortion is reduced by controlling the bias current 
of the LDs. For the typical results using this technique, see Table 5.1 in Section 5.3.

\subsubsection{Cascaded linearized modulator}

In this technique, shown in Figure 5.5, two modulators are used in cascade: the second modulator compensates for the nonlinearity generated by the first modulator. Suppression of the nonlinearities is achieved by adjusting the bias voltages of the two modulators and the relative amplitudes and phases of the two $\mathrm{RF}$ input signals to each port [13].

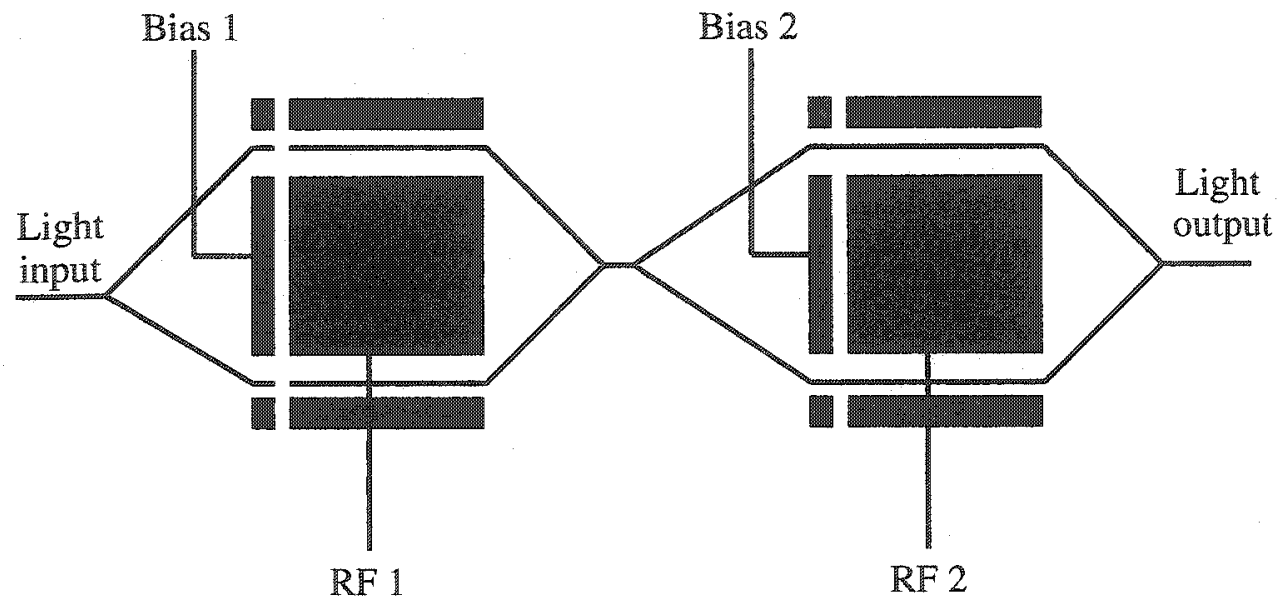

Figure 5.5: Schematic diagram for the cascaded linearized modulator [13].

\subsubsection{Post-distortion}

Post-distortion is a direct compensation technique that has no feedback or feed-forward paths [150]. In this technique, the modulated signal (laser output) is fed into a postdistortion circuit that introduces artificial distortion that cancels, as possible, the distortion introduced by the optical transmitter. Post-distortion can be added to the modulated signal path either in the optical domain (before the PD) as shown in Figure 5.6 or in the electrical domain (after the PD) as shown in Figure 5.7.

Post-distortion in wideband applications is rare, except for some theoretical studies in the 
literature [162-164].

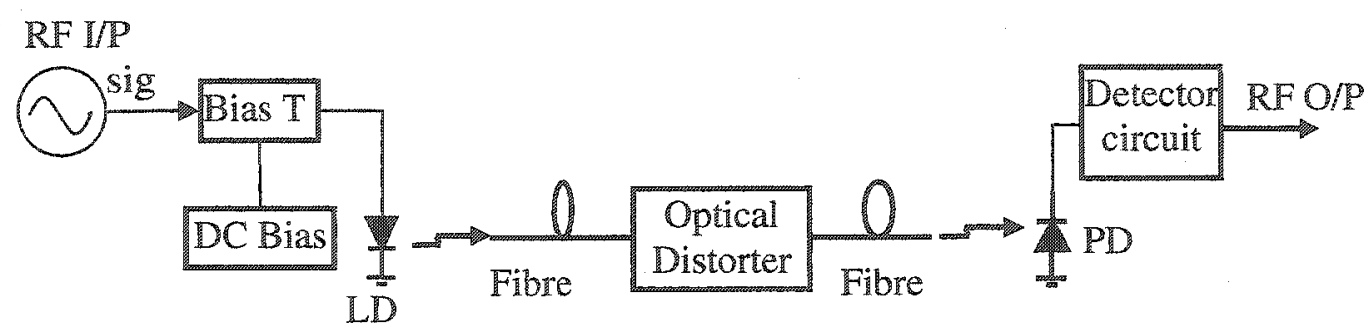

Figure 5.6: Optical domain post-distortion.

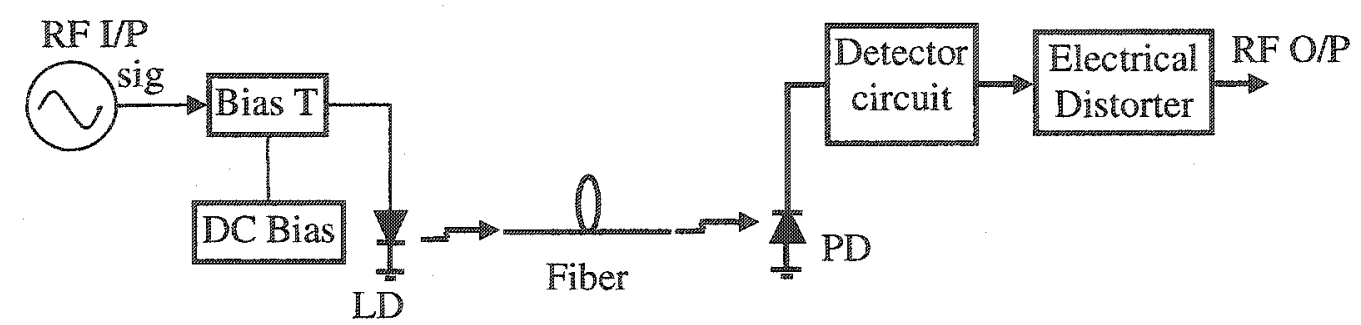

Figure 5.7: Electrical domain post-distortion.

\subsubsection{Pre-distortion linearizer}

The idea of the pre-distortion linearization technique is to insert an electronic pre-distorter in the signal path in front of the laser diode. The pre-distortion technique can be implemented using either analog circuits or digital circuits. A DSP can use known pre-distortion coefficients stored in a lookup table (LUT) to pre-distort the input signal. The hardware circuits or the coefficients in the digital pre-distorters are generated based on the known distortion characteristics of the laser diode. These distortion characteristics can be derived by analyzing the laser using either Volterra or Wiener theory. The basic structure of a predistortion implementation of $5^{\text {th }}$ order is shown in Figure 5.8. From this figure, the output of the laser diode is a nonlinear function of the input signal " $\mathrm{S}$ " and can be represented by a polynomial series $G 1 S+G 2 S^{2}+G 3 S^{3}+G 4 S^{5}, \mathrm{G} 1, \mathrm{G} 2, \mathrm{G} 3$ and $\mathrm{G} 4$ are functions of the 
laser parameters, $\phi 1, \phi 2, \phi 3$, and $\phi 4$ are phase adjusters. For the typical results using this technique, see Table 5.1 in Section 5.3. The above results support the use of pre-distortion

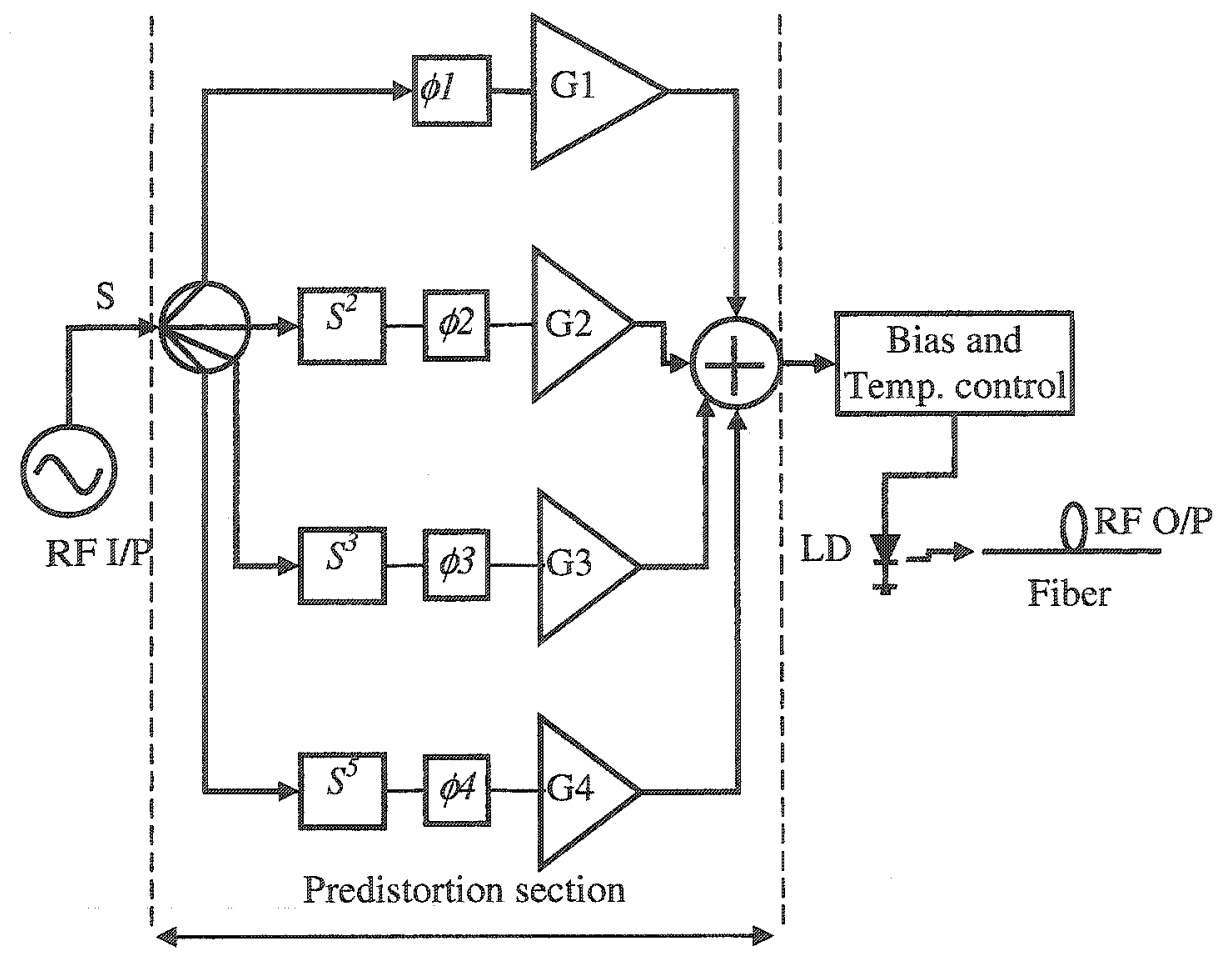

Figure 5.8: Predistortion system.

techniques in broadband applications. The inability to compensate for changes in the laser diode due to aging, thermal effects, or any differences due to the manufacturing is the major drawback of this technique as presented in the cited publications.

\subsubsection{Adaptive pre-distortion}

Adaptive pre-distortion uses the same idea of pre-distortion but it periodically senses the laser's output in order to update the coefficients in the DSP section (in the case of digital pre-distortion) or to change the gain of pre-distorter branches (in case of analogue circuits) to compensate for any of the laser's time-varying non-linearities due to aging, thermal effects and other effects. 
Figure 5.9 shows the general block diagram of the adaptive pre-distorter. In this figure second, third and ffth order harmonics of the input signal are generated by corresponding circuits. The feedback path is used to compensate for thermal effects, aging and any other changes. The photodiode in the feedback path is used to monitor the distortion levels. A higher order adaptive filter was proposed to compensate for laser nonlinearity in [149].

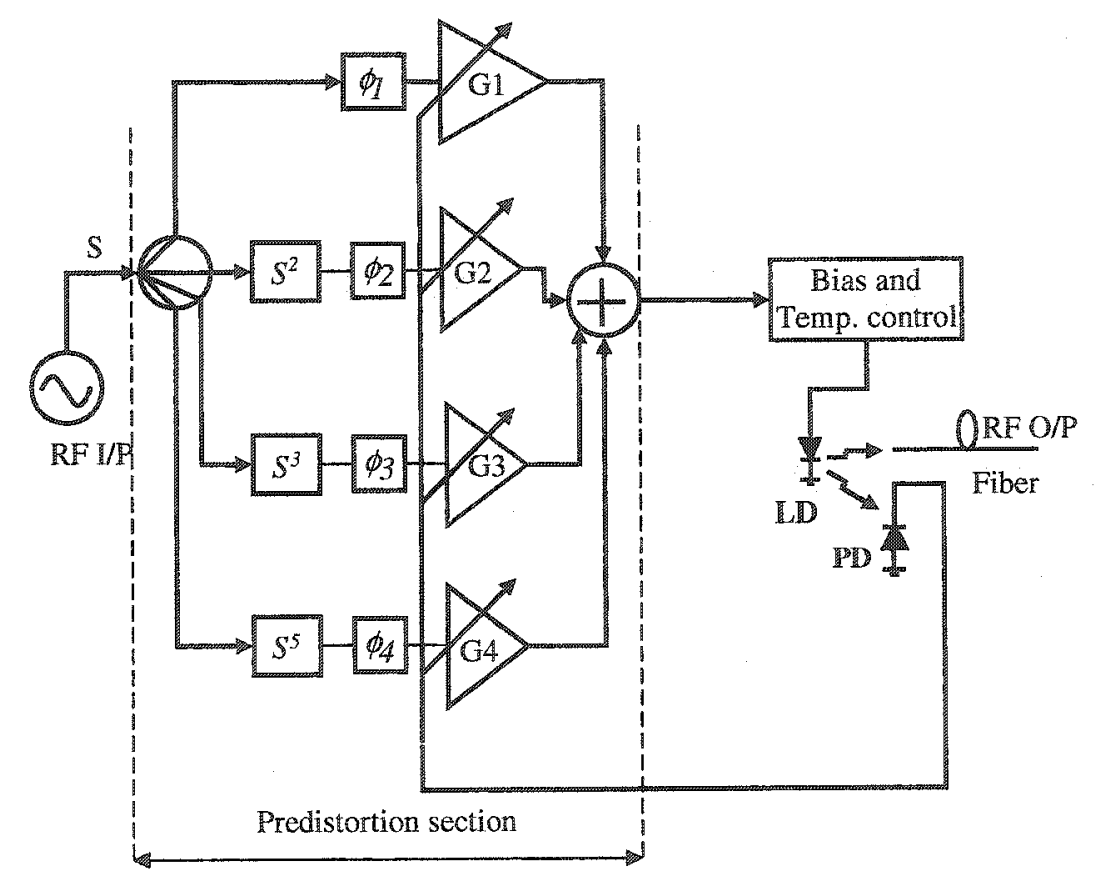

Figure 5.9: Block diagram of the adaptive pre-distortion system.

The proposed filter was adapted for distortions in vector modulated symbols and did not utilize in depth knowledge of the physical link parameters. Using this method, AM-AM compensation was achieved for a reduced dynamic range of $1 \mathrm{~mW}$, and the error between the original phase and the reduced phase was less than \pm 0.1 radian up to $2 \mathrm{~mW}$. In [149] it was demonstrated that a third order filter accurately models the ROF link while a second order filter adequately compensates for the phase nonlinearity. 


\subsection{Summary}

In this chapter we reviewed the concepts of nonlinearity in general and we also discussed the laser nonlinearity and distortion generation. As discussed in Section 5.2, various linearization techniques have been introduced to minimize the signal distortion due to the laser nonlinearity. For each technique, we discussed some of published results. Table 5.1 summarizes these techniques and some of the published results related to them.

Table 5.1: Summary of the linearization techniques and distortion reduction results.

\begin{tabular}{|c|c|c|}
\hline Technique & Typical results & Reference \\
\hline $\begin{array}{l}\text { Optoelectronic } \\
\text { feedback }\end{array}$ & $\begin{array}{l}\text { The } 2^{\text {nd }} \text { and } 3^{\text {rd }} \text { harmonic distortion were reduced } \\
\text { by } 10 \text { and } 12 \mathrm{~dB} \text { respectively for a } 6 \mathrm{MHz} \text { channel. } \\
\text { The } 2^{\text {nd }} \text { and } 3^{\text {rd }} \text { harmonic distortion were reduced } \\
\text { by } 12 \mathrm{~dB} \text { and } 4 \mathrm{~dB} \text { for a } 6 \mathrm{MHz} \text { channel. } \\
\mathrm{A} 20 \mathrm{~dB} \text { reduction of the second harmonic distor- } \\
\text { tion in a single channel TV transmission. }\end{array}$ & $\begin{array}{l}{[165]} \\
{[177]}\end{array}$ \\
\hline $\begin{array}{l}\text { Optical feed- } \\
\text { forward }\end{array}$ & $\begin{array}{l}30 \mathrm{~dB} \text { reduction in the adjacent channel interfer- } \\
\text { ence over } 900 \mathrm{MHz} \text { bandwidth. } \\
\text { Reduction in the second harmonic distortion of } \\
\text { more than } 14 \mathrm{~dB} \text { and intermodulation by more } \\
\text { than } 10 \mathrm{~dB} \text { over the frequency range of } 100 \mathrm{MHz}- \\
1.5 \mathrm{GHz} \text {. } \\
\text { Simultaneous reduction in the laser intensity noise } \\
\text { of more than } 10 \mathrm{~dB} \text { over the frequency range } 1.7- \\
3.0 \mathrm{GHz} \text {. } \\
\text { Reduction of third-order intermodulation distor- } \\
\text { tion of more than } 10 \mathrm{~dB} \text { over the frequency range } \\
2.1 \mathrm{GHz}-3.0 \mathrm{GHz} \text {. } \\
\text { Reduction of second order-harmonic distortion of } \\
25 \mathrm{~dB} \text { at } 2.4 \mathrm{GHz} \text {. } \\
\text { An average of } 20 \mathrm{~dB} \text { reduction over } 850 \mathrm{MHz} \text { range } \\
\text { of the second and third order harmonic and inter- } \\
\text { modulation distortion. } \\
\text { An average of } 10 \mathrm{~dB} \text { reduction of the laser noise } \\
\text { over a bandwidth of } 400 \mathrm{MHz} \text {. }\end{array}$ & $\begin{array}{l}{[161]} \\
{[11,159]}\end{array}$ \\
\hline $\begin{array}{l}\text { External light } \\
\text { injection }\end{array}$ & $\begin{array}{l}\text { A reduction of } 10 \mathrm{~dB} \text { in the second order harmonic } \\
\text { distortion. }\end{array}$ & {$[12,173]$} \\
\hline
\end{tabular}




\begin{tabular}{|c|c|c|}
\hline $\begin{array}{l}\text { Dual-parallel } \\
\text { modulation }\end{array}$ & $\begin{array}{l}\text { A } 15 \mathrm{~dB} \text { reduction in IMD3. } \\
\text { A } 23 \mathrm{~dB} \text { improvement in which the dynamic range } \\
\text { was increased by } 19.6 \mathrm{~dB}\end{array}$ & $\begin{array}{l}{[174]} \\
{[175]} \\
\end{array}$ \\
\hline $\begin{array}{l}\text { Cascaded lin- } \\
\text { earized mod- } \\
\text { ulator }\end{array}$ & $\begin{array}{l}\text { A } 34 \mathrm{~dB} \text { reduction in IP3 and SFDR of } 53 \mathrm{~dB} \text { for } \\
\text { a signal bandwidth of } 2 \mathrm{GHz} \text {. }\end{array}$ & {$[13]$} \\
\hline $\begin{array}{l}\text { Pre- } \\
\text { distortion } \\
\text { linearizer }\end{array}$ & $\begin{array}{l}\text { A } 15 \mathrm{~dB} \text { reduction in the second order harmonic } \\
\text { distortion (2HD) and a } 9 \mathrm{~dB} \text { reduction in the third } \\
\text { order harmonic distortion (3HD) over the range } \\
500 \mathrm{MHz}-2 \mathrm{GHz} \text { for a directly modulated laser } \\
\text { diode. } \\
\text { A reduction of nearly } 20 \mathrm{~dB} \text { in the } \mathrm{IP} 3 \text { for a } \\
32 \% \text { optical modulation index (OMI) and an } 11 \\
\mathrm{~dB} \text { improvement in Spurious-Free Dynamic Range } \\
\text { (SFDR) as a result of linearizing a Mach-Zehnder } \\
\text { modulator for external laser modulation. } \\
\text { A } 17 \mathrm{~dB} \text { suppression of the IP3 at for an OMI of } \\
49.6 \% \text { the frequency range from DC to } 1.3 \mathrm{GHz} \text {. } \\
\text { Measurements over an extended channel band- } \\
\text { width to the } 61^{\text {st }} \text { channel yield a } 10 \mathrm{~dB} \text { improve- } \\
\text { ment in the CSO over the range } 330-450 \mathrm{MHz} \text {. } \\
\text { A reduction of } 17,11 \text {, and } 7 \mathrm{~dB} \text { at channels } 3 \text {, } 11 \text {, } \\
\text { and } 40 \text { respectively with no change in the CNR or } \\
\mathrm{CTB} \text { using a second order pre-distorter. } \\
\text { A circuit was able to cancel the third- and fifth } \\
\text { order intermodulation distortion of a } 1.55 \mu m \text { DFB } \\
\text { laser integrated with electroabsorption modulator. }\end{array}$ & $\begin{array}{l}{[169]} \\
{[169]} \\
{[155]} \\
{[178,179]}\end{array}$ \\
\hline
\end{tabular}

The common aspect in all of the discussed linearization techniques is the lack of analytical model that can identify the levels of distortion due to the laser nonlinearity.

In conclusion, an accurate model that is able to predict the laser distortion and able to sense other changes in the laser diode due to aging, thermal effects, or any differences due to the manufacturing is needed. The above results support the use of adaptive pre-distorter that is based on this model in broadband applications. 


\section{Chapter 6}

\section{Pre-distorter Modeling, Implementation and Nonlinearity Evaluation}

\subsection{Inverse laser modeling}

A pre-distorter will be developed based on the inverse of the laser model such that when it is cascaded with the laser model it reduces the overall distortion.

The Volterra series gives an algebraically tractable method of calculating the laser's transfer function and its inverse. Laser rate equations represent an input-output relation between the injection current and the photon density $\mathbb{P}=f(I)$. Since the analytical solution of this equation is complicated, we will attempt to solve the model in an opposite way. This means we will derive the Volterra series of $I=f(\mathbb{P})$ instead of $\mathbb{P}=f(I)$, in order to determine the laser's inverse function. To do so, a frequency domain pre-distorter model will be derived, and then the time domain model will be derived from the frequency domain model. The pre-distorter circuit will be an implementation of the time domain Volterra series of the inverse laser model. The modified laser model presented in Chapter 4 is algebraically manipulated and denormalized and given in the form $I=f(\mathbb{P})$. From Equation (3.3.1), the carrier density can be given as,

$$
\mathbb{C}=\frac{\frac{d \mathbb{P}}{d t}+\frac{\mathbb{P}}{\tau_{p h}}+\frac{G \mathbb{C}_{t r} \mathbb{P}}{\sqrt{1+\mathbb{P} / \mathbb{P}_{s}}}}{\frac{\Gamma \beta_{s p}}{\tau_{e}}+\frac{G \mathbb{P}}{\sqrt{1+\mathbb{P} / \mathbb{P}_{s}}}}
$$


Substituting $G=\Gamma v_{g} \gamma_{o}, \mathbb{P}$ by $P$ where $P=P_{d c}+P_{a c}, \mathbb{P}_{s}$ by $P_{s}, d \mathbb{P} / d t$ by $P^{\prime}, \mathbb{C}_{t r}$ by $C_{t r}$, $\mathbb{C}$ by $C$ where $C=C_{d c}+C_{a c}$, and $I$ by $I_{d c}+I_{a c}$ in (6.1.1) yields,

$$
\left(C_{d c}+C_{a c}\right)=\frac{P^{\prime}+\frac{\left(P_{d c}+P_{a c}\right)}{\tau_{p h}}+\frac{G \mathbb{C}_{t r}\left(P_{d c}+P_{a c}\right)}{\sqrt{1+\left(P_{d c}+P_{a c}\right) / P_{s}}}}{\frac{\Gamma \beta_{s p}}{\tau_{e}}+\frac{G\left(P_{d c}+P_{a c}\right)}{\sqrt{1+\left(P_{d c}+P_{a c}\right) / P_{s}}}}
$$

The expansion, up to the third order, of $\left(1 / \sqrt{1+\left(P_{d c}+P_{a c}\right) / P_{s}}\right)$ using the Taylor series is given as,

$$
\frac{1}{\sqrt{1+\left(P_{d c}+P_{a c}\right) / P_{s}}} \equiv \Phi=M 0+M 1 P_{a c}+M 2 P_{a c}^{2}+M 3 P_{a c}^{3}
$$

in which $M 0, M 1, M 2, M 3$ are given by,

$$
\begin{aligned}
M 0 & =\frac{1}{1+P_{d c} / P_{s}} \\
M 1 & =\frac{-1}{2 P_{s}\left(1+P_{d c} / P_{s}\right)^{3 / 2}} \\
M 2 & =\frac{3}{8 P_{s}^{2}\left(1+P_{d c} / P_{s}\right)^{5 / 2}} \\
M 3 & =\frac{-5}{16 P_{s}^{3}\left(1+P_{d c} / P_{s}\right)^{7 / 2}}
\end{aligned}
$$

Expansion of (1/Den), where Den is the denominator of (6.1.2), up to the third order, and substitution of $\Gamma \beta_{s p} / \tau_{e}$ by co leads to,

$$
\frac{1}{\left(c o+G\left(P_{d c}+P_{a c}\right) \Phi\right)} \equiv \Psi=D 0+D 1 P_{a c}+D 2 P_{a c}^{2}+D 3 P_{a c}^{3}
$$

in which $D 0, D 1, D 2, D 3$ are given by,

$$
D 0=\frac{1}{c o+G M 0 P_{d c}}
$$




$$
\begin{aligned}
D 1= & \frac{-G}{c o^{2}+2 c o G M 0 P_{d c}+\left(G M 0 P_{d c}\right)^{2}}\left(M 0+M 1 P_{d c}\right) \\
D 2= & \frac{-G}{\left(c o+G M 0 P_{d c}\right)^{2}}\left(M 1+M 2 P_{d c}\right) \\
& +\frac{G^{2}\left(M 0^{2}+2 M 0 M 1 P_{d c}+M 1^{2} P_{d c}^{2}\right)}{\left(c o+G M 0 P_{d c}\right)\left(c o^{2}+2 c o G M 0 P_{d c}+G^{2} M 0^{2} P_{d c}^{2}\right)} \\
D 3= & \frac{G^{2}}{\left(c o+G M 0 P_{d c}\right)^{3}}\left(M 0 M 1+M 1^{2} P_{d c}+M 0 M 2 P_{d c}+M 1 M 2 P_{d c}^{2}\right) \\
& -\frac{G}{\left(c o+G M 0 P_{d c}\right)^{2}}\left(M 2+M 3 P_{d c}\right) \\
& -\frac{G^{3}\left(M 0^{3}+3 M 0^{2} M 1 P_{d c}+3 M 0 M 1^{2} P_{d c}^{2}+M 1^{3} P_{d c}^{3}\right)}{\left(c o+G M 0 P_{d c}\right)^{2}\left(c o^{2}+2 c o G M 0 P_{d c}+G^{2} M 0^{2} P_{d c}^{2}\right)} \\
& +\frac{G^{2}\left(M 0 M 1+M 1^{2} P_{d c}+M 0 M 2 P_{d c}+M 1 M 2 P_{d c}^{2}\right)}{\left(c o+G M 0 P_{d c}\right)\left(c o^{2}+2 c o G M 0 P_{d c}+G^{2} M 0^{2} P_{d c}^{2}\right)}
\end{aligned}
$$

Substitution of equations (6.1.3) and (6.1.5) into equation (6.1.2) gives the carrier density and its derivative as,

$$
\begin{aligned}
\left(C_{a c}+C_{d c}\right)= & Z_{0}+Z_{1} P_{a c}+Z_{2} P_{a c}^{\prime}+Z_{3} P_{a c}^{2}+Z_{4} P_{a c} P_{a c}^{\prime} \\
& +Z_{5} P_{a c}^{3}+Z_{6} P_{a c}^{2} P_{a c}^{\prime}+Z_{7} P_{a c}^{3} P_{a c}^{\prime} \\
C_{a c}^{\prime}= & Z_{1} P_{a c}^{\prime}+2 Z_{3} P_{a c} P_{a c}^{\prime}+Z_{4}\left(P_{a c}^{\prime}\right)^{2}+Z_{4} P_{a c} P_{a c}^{\prime \prime}+3 Z_{5} P_{a c}^{2} P_{a c}^{\prime} \\
& +2 Z_{6} P_{a c}\left(P_{a c}^{\prime}\right)^{2}+Z_{6} P_{a c}^{2} P_{a c}^{\prime \prime}+3 Z_{7} P_{a c}^{2}\left(P_{a c}^{\prime}\right)^{2}+Z_{7} P_{a c}^{3} P_{a c}^{\prime \prime}
\end{aligned}
$$

in which,

$$
\begin{aligned}
Z 0= & D 0 P_{d c}\left(C_{t r} G M 0+1 / \tau_{p h}\right) \\
Z 1= & D 0 G C_{t r}\left(M 0+M 1 P_{d c}\right)+D 0 / \tau_{p h} \\
& +D 1 P_{d c}\left(G M 0 C_{t r}+1 / \tau_{p h}\right) \\
Z 2= & D 0 \\
Z 3= & D 1\left(G C_{t r}\left(M 0+M 1 P_{d c}\right)+1 / \tau_{p h}\right) \\
& +D 0 G C_{t r}\left(M 1+M 2 P_{d c}\right)+D 2 P_{d c}\left(G M 0 C_{t r}+1 / \tau_{p h}\right) \\
Z 4= & D 1
\end{aligned}
$$




$$
\begin{aligned}
Z 5= & D 0 G C_{t r}\left(M 2+M 3 P_{d c}\right)+D 1 G C_{t r}\left(M 1+M 2 P_{d c}\right) \\
& +D 2\left(G C_{t r}\left(M 0+M 1 P_{d c}\right)+\left(1 / \tau_{p h}\right)\right) \\
& +D 3 P_{d c}\left(G C_{t r} M 0+\left(1 / \tau_{p h}\right)\right) \\
Z 6= & D 2 \\
Z 7= & D 3
\end{aligned}
$$

Using (6.1.3)-(6.1.19) in equation (3.3.2), the modulating current is given as,

$$
I_{a c}=-I_{d c}+q V_{c} C_{a c}^{\prime}+\frac{\left(C_{a c}+C_{d c}\right)}{\tau_{e}}+g_{m}\left(\left(C_{a c}+C_{d c}\right)-C_{t r}\right) \times \Phi
$$

Where $g_{m}=G / \Gamma$. Substitution of (6.1.10), (6.1.11) and (6.1.3) into (6.1.20) and neglecting terms higher than the third order leads to the following,

$$
\begin{aligned}
I_{a c}= & K \\
& +\left\{m_{0} P_{a c}+m_{1} P_{a c}^{\prime}+m_{2} P_{a c}^{\prime \prime}\right\} \\
& +\left\{m_{3} P_{a c}^{2}+m_{1} P_{a c} P_{a c}^{\prime}+m_{5}\left(P_{a c}^{\prime}\right)^{2}+m_{5} P_{a c} P_{a c}^{\prime \prime}\right\} \\
& +\left\{m_{6} P_{a c}^{3}+m_{7} P_{a c}^{2} P_{a c}^{\prime}+2 m_{8} P_{a c}\left(P_{a c}^{\prime}\right)^{2}+m_{8} P_{a c}^{2} P_{a c}^{\prime \prime}\right\}
\end{aligned}
$$

$K$ in (6.1.21a) is a constant, (6.1.21b) is of first order in the series, (6.1.21c) is of second order in the series and (6.1.21d) is of third order in the series. The constants $m_{0} \ldots m_{8}$ are functions of the laser parameters and are given by,

$$
\begin{aligned}
m_{0}= & g_{m} q V_{c}\left(M 0 Z 0-M 0 C_{t r}+M 1 P_{d c} Z 0+M 0 P_{d c} Z 1\right. \\
& \left.-M 1 C_{t r} P_{d c}\right)+\frac{q V_{c} Z 1}{\tau_{e}} \\
m_{1}= & q V_{c}\left(Z 1+g_{m} M 0 P_{d c} Z 2+\frac{Z 2}{\tau_{e}}\right) \\
m_{2}= & q V_{c} Z 2 \\
m_{3}= & g_{m} q V_{c}\left(-C_{t r}\left(M 1+M 2 P_{d c}\right)+Z_{0}\left(M 1+M_{2} P_{d c}\right)\right. \\
& \left.+Z 1\left(M 0+M 1 P_{d c}\right)+M 0 P_{d c} Z 3\right)+\frac{q V_{c} Z_{3}}{\tau_{e}}
\end{aligned}
$$




$$
\begin{aligned}
m_{4}= & q V_{c}\left(g_{m}\left(M 0 Z 2+M 1 P_{d c} Z 2\right)+2 Z 3+g_{m} M 0 P_{d c} Z_{4}+\frac{Z_{4}}{\tau_{e}}\right)( \\
m_{5}= & q V_{c} Z_{4} \\
m_{6}= & g_{m} q V_{c}\left(-C_{t r}\left(M 2+M 3 P_{d c}\right)+Z 0\left(M 2+M 3 P_{d c}\right)\right. \\
& +Z 1\left(M 1+M 2 P_{d c}\right)+Z 3\left(M 0+M 1 P_{d c}\right) \\
& \left.+Z 5 M 0 P_{d c}\right)+\frac{q V_{c} Z_{5}}{\tau_{e}} \\
m_{7}= & q V_{c}\left(g_{m} M 1\left(Z 2+P_{d c} Z 4\right)+3 Z 5+g_{m} M 0 P_{d c} Z 6\right. \\
& \left.+g_{m} M 2 P_{d c} Z 2+G_{m} M 0 Z 4+\frac{Z_{6}}{\tau_{e}}\right) \\
m_{8}= & q V_{c} Z_{6}
\end{aligned}
$$

Equation (6.1.21) represents the input-output relation (the laser's inverse model) that will be used for the extraction of the pre-distorter Volterra kernels.

\subsection{Frequency domain laser's pre-distorter Volterra model}

To extract the Volterra kernels, the harmonic input method presented in Section F.3.1 in Appendix $F$ is applied to equation (6.1.21) as follows:

1. The first order Volterra transfer function is the coefficient of $e^{j \omega_{1} t}$ if one tone input of the form $P_{a c}=P_{d c} e^{j \omega_{1} t}$ is applied to $(6.1 .21)$ and is given as,

$$
G_{1}\left(\omega_{1}\right)=\left(m_{0}+m_{1} j \omega_{1}-m_{2} \omega_{1}^{2}\right) P_{d c}
$$

2. The second order Volterra transfer function is the coefficient of $e^{j\left(\omega_{1}+\omega_{2}\right) t}$ if two tone inputs of the form $P_{a c}=P_{d c} e^{j \omega_{1} t}+P_{d c} e^{j \omega_{2} t}$ are applied to (6.1.21) and is given as,

$$
G_{2}\left(\omega_{1}, \omega_{2}\right)=\left(2 m_{3}+j m_{4}\left(\omega_{1}+\omega_{2}\right)-m_{5}\left(\omega_{1}+\omega_{2}\right)^{2}\right) P_{d c}^{2}
$$


3. The third order Volterra transfer function is the coefficient of $e^{j\left(\omega_{1}+\omega_{2}+\omega_{3}\right) t}$ if three tone inputs of the form of $P_{a c}=P_{d c} e^{j \omega_{1} t}+P_{d c} e^{j \omega_{2} t}+P_{d c} e^{j \omega_{3} t}$ are applied to (6.1.21) and is given as,

$$
G_{3}\left(\omega_{1}, \omega_{2}, \omega_{3}\right)=\left(6 m_{6}+2 j m_{7}\left(\omega_{1}+\omega_{2}+\omega_{3}\right)-2 m_{8}\left(\omega_{1}+\omega_{2}+\omega_{3}\right)^{2}\right) P_{d c}^{3}
$$

Equations (6.2.1), (6.2.2) and (6.2.3) represent the pre-distorter frequency domain Volterra transfer functions.

\subsection{Time domain pre-distorter Volterra models}

The time domain pre-distorter model can be derived from the frequency domain model using the direct expansion method presented in Section $\mathbb{F} .3 .2$. For simplicity, equations $(6.2 .1),(6.2 .2)$ and $(6.2 .3)$ can be represented by its Laplace transform if each $j \omega$ is replaced by " $s$ " as follows,

$$
\begin{aligned}
G_{1 L}\left(s_{1}\right) & =\left(m_{0}+m_{1} s_{1}+m_{2} s_{1}^{2}\right) P_{d c} \\
G_{2 L}\left(s_{1}, s_{2}\right) & =\left(2 m_{3}+m_{4}\left(s_{1}+s_{2}\right)+m_{5}\left(s_{1}+s_{2}\right)^{2}\right) P_{d c}^{2} \\
G_{3 L}\left(s_{1}, s_{2}, s_{3}\right) & =\left(6 m_{6}+2 m_{7}\left(s_{1}+s_{2}+s_{3}\right)+2 m_{8}\left(s_{1}+s_{2}+s_{3}\right)^{2}\right) P_{d c}^{3}
\end{aligned}
$$

Each branch of (6.3.1) consists of three terms:

- The first term can be represented by an amplifier with gain $m_{0}$ for the first branch, $2 m_{3}$ for the second branch and $6 m_{6}$ for the third branch.

- The second terms can be represented by a differentiator and an amplifier with gain. $m_{1}$ for the first branch, $m_{4}$ for the second branch and $2 m_{7}$ for the third branch.

- The last term can be represented by cascaded (tow) differentiators and an amplifier with gain $m_{2}$ for the first branch, $m_{5}$ for the second branch and $2 m_{8}$ for the third branch.

Each branch of (6.3.1) is represented as follows: 
1. The first order Volterra system of the pre-distorter is represented by equation (6.3.1a) and is illustrated in Figure 6.1

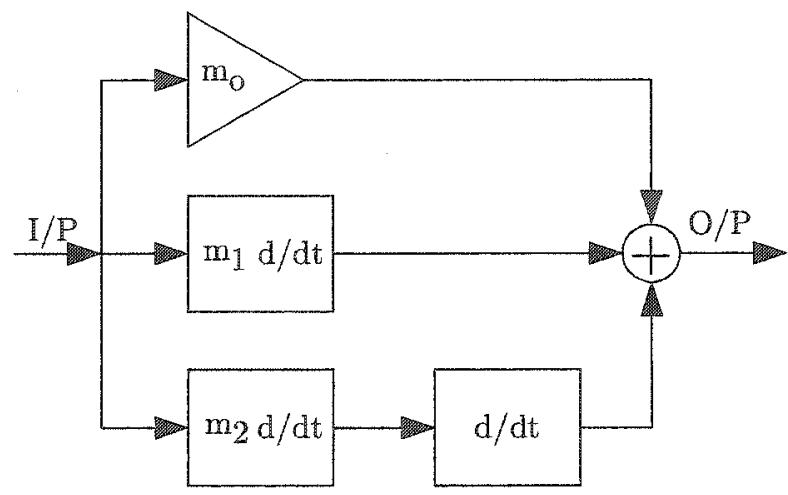

Figure 6.1: A first order Volterra system representation of the pre-distorter.

2. The second order Volterra system representation of the pre-distorter is derived by comparing equation (6.3.1b) with (F.3.3). Relations (6.3.1b) and (F.3.3) can be considered similar if we assume $G_{x}\left(s_{1}\right)=G_{y}\left(s_{2}\right)=1$. Thus, the second order Volterra system of the pre-distorter can be represented by the block diagram shown in Figure 6.2.

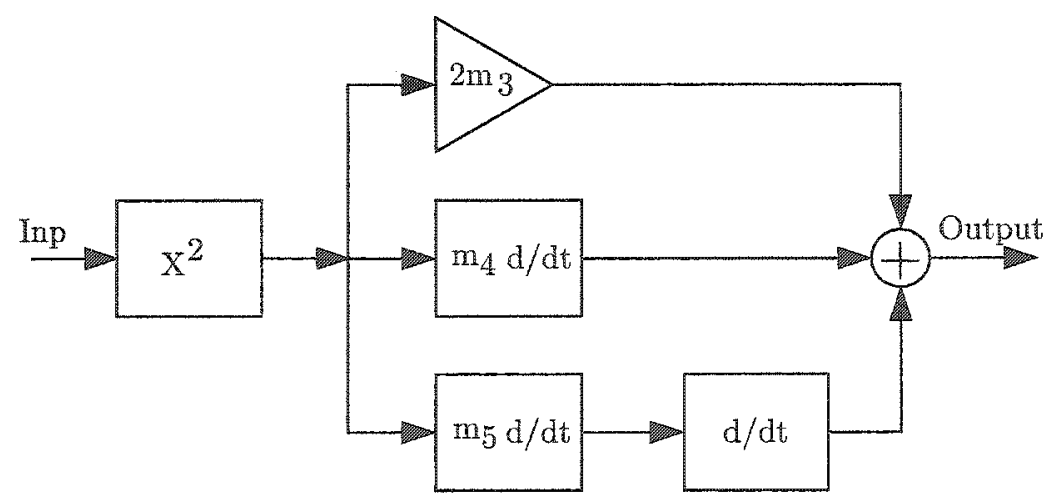

Figure 6.2: A second order Volterra system representation of the pre-distorter.

3. The third order Volterra system of the pre-distorter is derived by comparing equation (6.3.1c) with (F.3.4). Relations (6.3.1b) and (F.3.4) can be considered similar if 
we assume $G_{x}\left(s_{1}\right)=G_{y}\left(s_{2}\right)=G_{k}\left(s_{3}\right)=G_{z}\left(s_{1}, s_{2}\right)=1$. Thus, the third order Volterra system of the pre-distorter can be represented by the block diagram shown in Figure 6.3

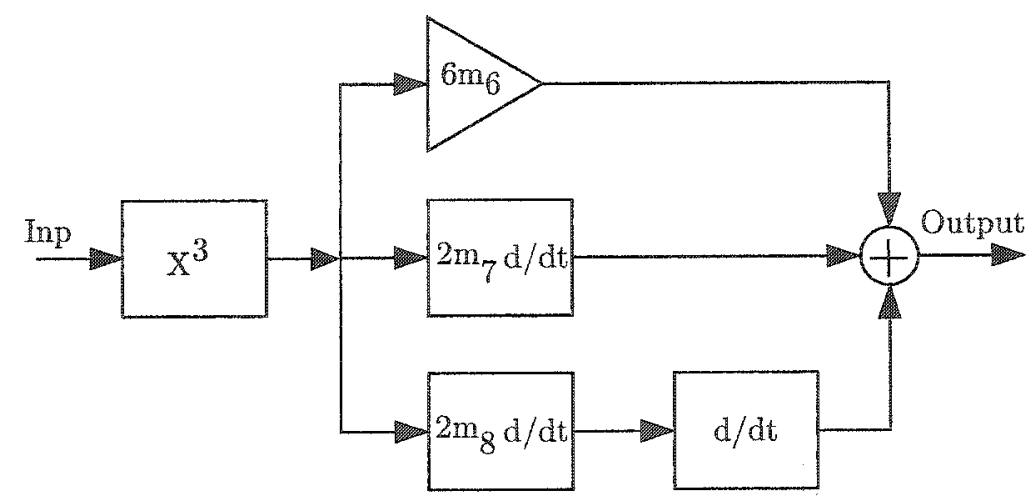

Figure 6.3: A third order Volterra system representation of the pre-distorter.

Based on the above discussion, the complete time domain Volterra system to $3^{\text {rd }}$ order of the pre-distorter model can be illustrated in Figure 6.4.

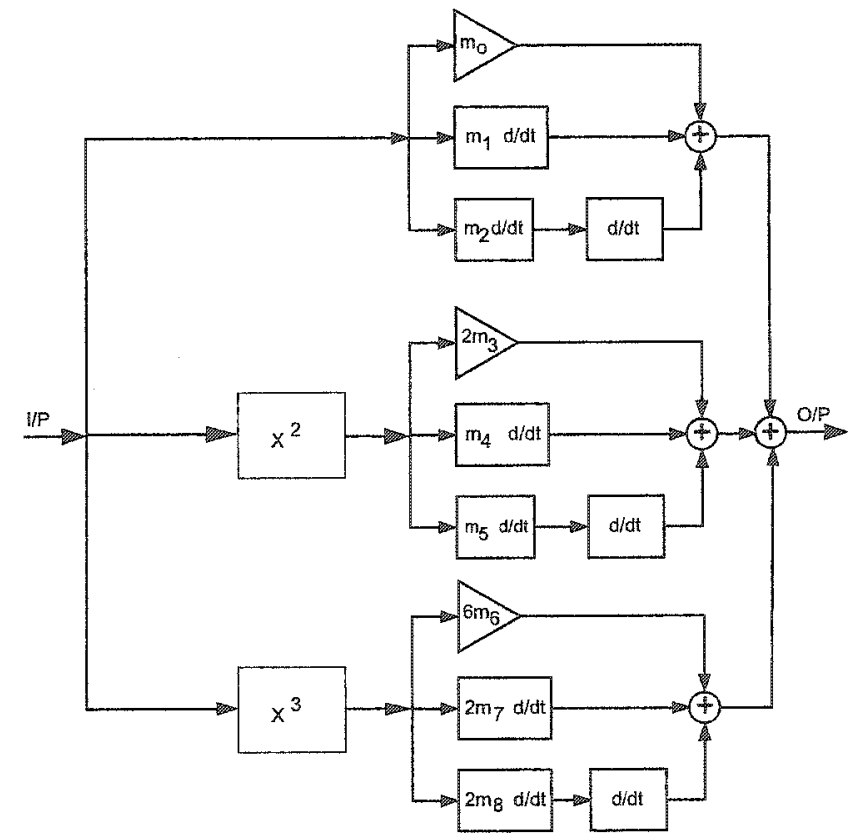

Figure 6.4: Entire block diagram of the the pre-distorter model represented by the Volterra system in time domain. 


\subsection{Proposed linearization technique and model}

Different laser diodes linearization techniques were reviewed in Chapter 5. From this review we concluded that no technique is suitable for all applications. The cited linearization methods don't account for the laser heating and aging. For our intended applications (broadband transmission over fibre), an adaptive linearization technique is the most suitable technique.

A block diagram of the proposed analog adaptive pre-distorter circuit is shown in Figure 6.5. The adaptive pre-distortion circuit is intended to cancel up to the third order intermodulation products. It consists of three branches; each branch represents an order of the Volterra system of the pre-distorter and is derived from the inverse Volterra series of the laser diode. The first branch represents the first order Volterra transfer function $\left(1^{s t}\right)$, the second branch represents the $2^{\text {nd }}$ order Volterra transfer function, and the third branch represents the $3^{\text {rd }}$ order Volterra transfer function. Coefficients $m_{o}, \ldots, m_{8}$ are the weighting functions of the pre-distorter. They are functions of the laser device parameters as given in (6.1.22). $\phi_{1}, \ldots, \phi_{3}$ are the phase adjusters used for each path.

To compensate for unpredictable changes in the laser diodes due to thermal effects, aging, or any other effects a photodiode in the feedback bath monitors the distortion levels from reference signals at frequencies $f_{1}$ and $f_{2}$. The DSP processor correlates the IMD monitored by the photodiode with reference signals of frequencies $f_{2}-f_{1}$ and $f_{2}-2 f_{1}$, and calculates the correct pre-distortion parameters that minimize the distortion based on this comparison. The DSP adjusts either the parameters in each branch or the gain unit of the whole branch. 


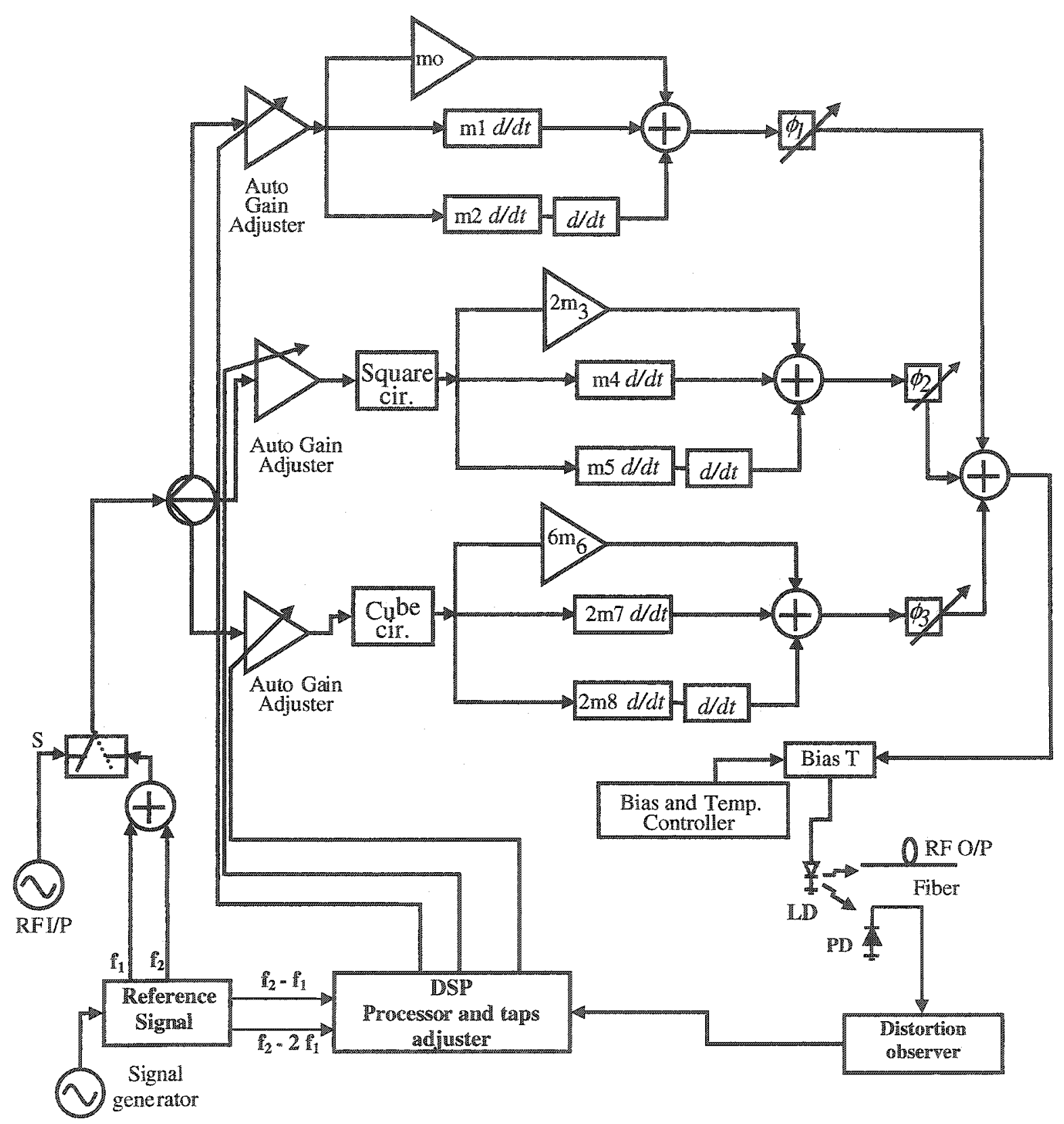

Figure 6.5: Schematic diagram for the proposed adaptive pre-distortion circuit. 


\subsection{Pre-distorter implementation and nonlinearity eval- uation}

We have proposed an electronic scheme that can compensate the signal distortion due to the laser nonlinearity. To examine the performance of the proposed distortion compensation scheme, an input signal that consists of one carrier (for harmonic distortion) or the sum of two, three or more carriers (for intermodulation distortion) can be applied to the input of the pre-distorter Volterra model shown in Figure 6.4. The pre-distorter should be able to modify the input RF signals such that they will be able to reduce the signal distortion levels due to the laser nonlinearity. To examine this procedure, the frequency domain pre-distorter model as given in (6.2.1) to (6.2.3) was placed in cascade with the frequency domain laser Volterra model. The distortion with and without the pre-distorter was examined. The derivation of the frequency domain laser Volterra model is presented in Section 6.5.1 while the distortion evaluation with and without the pre-distorter is discussed in Section 6.5 .2

\subsubsection{Frequency domain semiconductor laser Volterra model}

Frequency domain pre-distorter and laser models enable evaluation of the distortion and pre-distortion. The laser Volterra model can be derived by following the same procedure used to derive the pre-distorter Volterra model. Starting with the laser nonlinear impulse response, the transfer functions representing the laser model in the frequency domain can be evaluated using the harmonic input method presented in Section F.3.1. The laser Volterra model can also be directly evaluated from the pre-distorter frequency domain Volterra model. Since the pre-distorter model is the inverse of the laser model, the laser frequency domain Volterra model is simply the inverse of the pre-distorter model. In this section the latter technique is used to derive the laser Volterra model in frequency domain. Using the 
pre-distorter frequency domain Volterra model in $(6.2 .1)-(6.2 .3)$, the transfer functions representing the laser model can be determined as follows,

- The first order transfer function (TF) is the inverse of equation (6.2.1),

$$
H_{1}(w)=\frac{1}{G_{1}(w)}
$$

The block diagram shown in Figure 6.6 represents the pre-distortion Volterra model and the laser model,

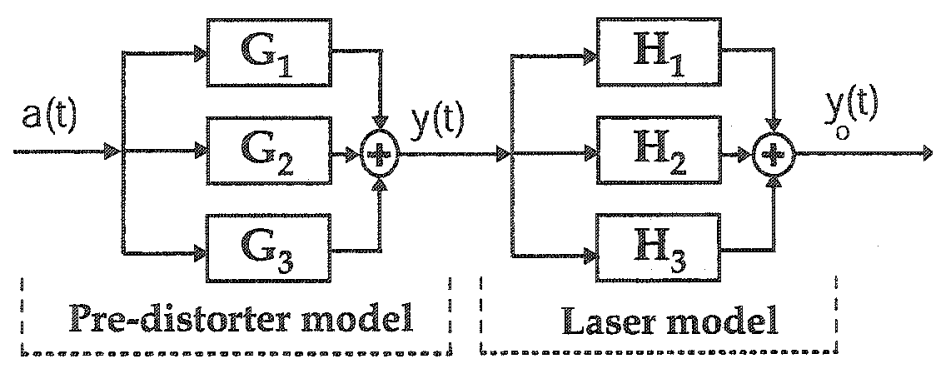

Figure 6.6: Block digram representation of the Volterra transfer functions representing the predistorter and laser models.

- The second order transfer function can be determined by assuming the input signal, $a(t)$, to the pre-distorter takes the form $a(t)=e^{j \omega_{1} t}+e^{j \omega_{2} t}$. The output of the pre-distorter $y(t)$ is given as,

$$
y(t)=G_{1}\left(\omega_{1}\right) e^{j \omega_{1} t}+G_{1}\left(\omega_{2}\right) e^{j \omega_{2} t}+G_{2}\left(\omega_{1}, \omega_{2}\right) e^{j\left(\omega_{1}+\omega_{2}\right) t}
$$

$y(t)$ is used as an input to the laser model. The overall response $y_{o}(t)$ can be deter- 
mined as follows,

$$
\begin{aligned}
y_{o}(t)= & H_{1}\left(\omega_{1}\right) G_{1}\left(\omega_{1}\right) e^{j \omega_{1} t}+H_{1}\left(\omega_{2}\right) G_{1}\left(\omega_{2}\right) e^{j \omega_{2} t} \\
& +H_{1}\left(\omega_{1}+\omega_{2}\right) G_{2}\left(\omega_{1}, \omega_{2}\right) e^{j\left(\omega_{1}+\omega_{2}\right) t} \\
& +H_{2}\left(\omega_{1}, \omega_{2}\right) G_{1}\left(\omega_{1}\right) G_{1}\left(\omega_{2}\right) e^{j\left(\omega_{1}+\omega_{2}\right) t} \\
& +H_{2}\left(\omega_{1}, \omega_{1}+\omega_{2}\right) G_{1}\left(\omega_{1}\right) G_{2}\left(\omega_{1}, \omega_{2}\right) e^{j\left(2 \omega_{1}+\omega_{2}\right) t} \\
& +H_{2}\left(\omega_{2}, \omega_{1}+\omega_{2}\right) G_{1}\left(\omega_{2}\right) G_{2}\left(\omega_{1}, \omega_{2}\right) e^{j\left(\omega_{1}+2 \omega_{2}\right) t}
\end{aligned}
$$

Since Volterra techniques treat each sub-system as a linear system, the overall response $y_{o}(t)$ can be reduced to the second order transfer function of the laser model if we equate the coefficient of $e^{j\left(\omega_{1}+\omega_{2}\right) t}$ to zeros, yielding,

$$
H_{2}\left(\omega_{1}, \omega_{2}\right)=-\frac{H_{1}\left(\omega_{1}+\omega_{2}\right) G_{2}\left(\omega_{1}, \omega_{2}\right)}{G_{1}\left(\omega_{1}\right) G_{1}\left(\omega_{2}\right)}
$$

Using equation (6.5.1) and replacing $H_{1}\left(\omega_{1}\right)$ with $1 / G_{1}\left(\omega_{1}\right)$, and $H_{1}\left(\omega_{1}, \omega_{2}\right)$ with $1 / G_{1}\left(\omega_{1}+\omega_{2}\right)$ in equation (6.5.4) gives,

$$
H_{2}\left(\omega_{1}, \omega_{2}\right)=-\frac{G_{2}\left(\omega_{1}, \omega_{2}\right)}{G_{1}\left(\omega_{1}\right) G_{1}\left(\omega_{2}\right) G_{1}\left(\omega_{1}+\omega_{2}\right)}
$$

- The third order transfer function can be determined by assuming the input signal, $a(t)$, to the pre-distorter takes the form $a(t)=e^{j \omega_{1} t}+e^{j \omega_{2} t}+e^{j \omega_{3} t}$. The output of the pre-distorter $y(t)$ is given as,

$$
\begin{aligned}
y(t)= & G_{1}\left(\omega_{1}\right) e^{j \omega_{1} t}+G_{1}\left(\omega_{2}\right) e^{j \omega_{2} t}+G_{3}\left(\omega_{3}\right) e^{j \omega_{3} t} \\
& +G_{2}\left(\omega_{1}, \omega_{2}\right) e^{j\left(\omega_{1}+\omega_{3}\right) t}+G_{2}\left(\omega_{1}, \omega_{3}\right) e^{j\left(\omega_{1}+\omega_{3}\right) t} \\
& +G_{2}\left(\omega_{2}, \omega_{2}\right) e^{j\left(\omega_{2}+\omega_{2}\right) t}+G_{3}\left(\omega_{1}, \omega_{3}, \omega_{3}\right) e^{j\left(\omega_{1}+\omega_{3}+\omega_{3}\right) t}
\end{aligned}
$$

where $y(t)$ is used as the input to the laser model. The overall response $y_{0}(t)$ can be 
determined as follows,

$$
\begin{aligned}
y_{0}(t)= & H_{1}\left(\omega_{1}\right) G_{1}\left(\omega_{1}\right) e^{j \omega_{1} t}+H_{1}\left(\omega_{2}\right) G_{1}\left(\omega_{2}\right) e^{j \omega_{2} t}+H_{1}\left(\omega_{3}\right) G_{1}\left(\omega_{3}\right) e^{j \omega_{3} t} \\
& +H_{1}\left(\omega_{1}+\omega_{2}\right) G_{2}\left(\omega_{1}, \omega_{2}\right) e^{j\left(\omega_{1}+\omega_{2}\right) t}+H_{1}\left(\omega_{1}+\omega_{3}\right) G_{2}\left(\omega_{1}, \omega_{3}\right) e^{j\left(\omega_{1}+\omega_{3}\right) t} \\
& +H_{1}\left(\omega_{2}+\omega_{3}\right) G_{2}\left(\omega_{2}, \omega_{3}\right) e^{j\left(\omega_{2}+\omega_{3}\right) t} \\
& +H_{1}\left(\omega_{1}+\omega_{2}+\omega_{3}\right) G_{3}\left(\omega_{1}, \omega_{2}, \omega_{3}\right) e^{j\left(\omega_{1}+\omega_{2}+\omega_{3}\right) t} \\
& +H_{2}\left(\omega_{1}, \omega_{2}+\omega 3\right) G_{1}\left(\omega_{1}\right) G_{2}\left(\omega_{2}, \omega_{3}\right) e^{j\left(\omega_{1}+\omega_{2}+\omega_{3}\right) t} \\
& +H_{2}\left(\omega_{2}, \omega_{1}+\omega_{3}\right) G_{1}\left(\omega_{2}\right) G_{2}\left(\omega_{1}, \omega_{3}\right) e^{j\left(\omega_{1}+\omega_{2}+\omega_{3}\right) t} \\
& +H_{2}\left(\omega_{3}, \omega_{1}+\omega_{2}\right) G_{1}\left(\omega_{3}\right) G_{2}\left(\omega_{1}, \omega_{2}\right) e^{j\left(\omega_{1}+\omega_{2}+\omega_{3}\right) t} \\
& +H_{3}\left(\left(\omega_{1}, \omega_{2}, \omega_{3}\right) G_{1}\left(\omega_{1}\right) G_{1}\left(\omega_{2}\right) G_{1}\left(\omega_{3}\right) e^{j\left(\omega_{1}+\omega_{2}+\omega_{3}\right) t}\right.
\end{aligned}
$$

The overall response $y_{o}(t)$ can be reduced to the third order transfer function of the laser model if we equate the coefficient of $e^{j\left(\omega_{1}+\omega_{2}+\omega_{3}\right) t}$ to zeros and make use of equations (6.5.1) and (6.5.5), yielding,

$$
\begin{aligned}
H_{3}\left(\left(\omega_{1}, \omega_{2}, \omega_{3}\right)=\right. & \frac{-1}{G_{1}\left(\omega_{1}\right) G_{1}\left(\omega_{2}\right) G_{1}\left(\omega_{3}\right) G_{1}\left(\omega_{1}+\omega_{2}\right)+\omega_{3}}\left\{G_{3}\left(\omega_{1}, \omega_{2}, \omega_{3}\right)\right. \\
& -\frac{G_{2}\left(\omega_{1}, \omega_{2}+\omega_{3}\right) G_{2}\left(\omega_{2}, \omega_{3}\right)}{G_{1}\left(\omega_{2}+\omega_{3}\right)}-\frac{G_{2}\left(\omega_{2}, \omega_{1}+\omega_{3}\right)}{G_{1}\left(\omega_{1}+\omega_{3}\right)} \\
& \left.-\frac{G_{2}\left(\omega_{3}, \omega_{1}+\omega_{2}\right)}{G_{1}\left(\omega_{1}+\omega_{2}\right)}\right\}
\end{aligned}
$$

Equations (6.5.1), (6.5.5), and (6.5.8) represent the laser frequency domain Volterra transfer functions.

\subsubsection{Distortion evaluation}

The response of the laser with and without the pre-distorter was calculated using the general formulations of a typical Volterra series in [180]. For simplicity, take the input $x(t)$ 
to be a sum of $n$ sinusoidal signals and given as,

$$
\begin{aligned}
x(t) & =e^{j \omega_{1} t}+e^{j \omega_{2} t}+\ldots+e^{j \omega_{n} t} \\
& =\frac{1}{2 \pi} \int_{-\infty}^{\infty} e^{j \omega t} X(\omega) d \omega
\end{aligned}
$$

in which $X(f)$ is the Fourier transform of $x(t) . f_{1}, \ldots, f_{n}$ are linearly independent. The resulting output from a $k^{\text {th }}$ order Volterra system in the frequency domain, subjected to an input $x(t)$, is given by,

$$
\begin{aligned}
Y(\omega)= & \frac{1}{1 !} Z_{1}\left(\omega_{1}\right) X\left(\omega_{1}\right)+\frac{1}{2 ! 2 \pi} \int_{-\infty}^{\infty} Z_{2}\left(\omega_{2}, \omega-\omega_{2}\right) X\left(\omega_{2}\right) X\left(\omega-\omega_{2}\right) d \omega_{2} \\
& +\frac{1}{3 !(2 \pi)^{2}} \int_{-\infty}^{\infty} d \omega_{2} \int_{-\infty}^{\infty} d \omega_{3} Z_{3}\left(\omega_{2}, \omega_{3}, \omega-\omega_{2}-\omega_{3}\right) X\left(\omega_{2}\right) X\left(\omega_{3}\right) X\left(\omega-\omega_{2}-\omega_{3}\right) \\
& +\ldots \\
& +\frac{1}{k !(2 \pi)^{(k-1)}} \int_{-\infty}^{\infty} \ldots \int_{-\infty}^{\infty} Z_{k}\left(\omega_{2}, \omega_{3}, \ldots, \omega-\omega_{2}-\omega_{3}-\cdots-\omega_{k}\right) \times \\
& X\left(\omega_{2}\right) X\left(\omega_{3}\right) \ldots X\left(\omega-\omega_{2}-\omega_{3}-\ldots-\omega_{k}\right) d \omega_{2} \ldots d \omega_{k}
\end{aligned}
$$

in which $Z_{n}\left(\omega_{1}, \omega_{2}-\omega_{3}-\cdots-\omega_{k}\right)$ refer to the $k^{t h}$-order Volterra transfer function.

\subsubsection{Distortion evaluation without using pre-distorter}

The distortion levels of the laser diode were determined in the frequency domain by applying an input signal current that consists of one carrier (for harmonic distortion) or the sum of two, three or more carriers (for intermodulation distortion) to the input of laser Volterra transfer functions given by (6.5.1), (6.5.5), and (6.5.8). The input signal current that consists of $m$ carriers is given by (6.5.12) in the time domain while its spectrum is 
given by (6.5.13) in frequency domain.

$$
\begin{aligned}
I(t) & =\frac{1}{2} \sum_{i=1}^{m}\left\{a_{i} e^{j 2 \pi f_{i} t}+a_{i}^{*} e^{-j 2 \pi f_{i} t}\right\} \\
I(\omega) & =\frac{1}{2(2 \pi)} \sum_{i=1}^{m}\left\{A_{i}\left(\omega-\omega_{i}\right)+A_{i}^{*}\left(-\omega-\omega_{i}\right)\right\} \\
& =\frac{1}{2(2 \pi)} \sum_{i=-m}^{m} A_{i}\left(\omega-\omega_{i}\right)
\end{aligned}
$$

Equation (6.5.11) was applied to the laser Volterra model by replacing each $Z(\omega)$ by its corresponding laser transfer function $H(\omega)$. The input $X(\omega)$ is equivalent to the input current $I(\omega)$. For a unit impulse, the response of the laser model represented by $(6.5 .1)$, (6.5.5), and (6.5.8) can be determined as follows,

$$
\begin{aligned}
Y(\omega)= & \frac{1}{1 !} H_{1}\left(\omega_{1}\right) \delta\left(\omega-\omega_{1}\right) \\
& +\frac{1}{2 ! 2 \pi} \int_{-\infty}^{\infty} H_{2}\left(\omega_{2}, \omega-\omega_{2}\right) \delta\left(\omega_{2}-\omega_{1}\right) \delta\left(\omega-\omega_{1}-\omega_{2}\right) d \omega_{2} \\
& +\frac{1}{3 !(2 \pi)^{2}} \int_{-\infty}^{\infty} \int_{-\infty}^{\infty} H_{3}\left(\omega_{3}, \omega_{2}, \omega-\omega_{2}-\omega_{3}\right) \times \\
& \delta\left(\omega_{3}-\omega_{1}\right) \delta\left(\omega_{2}-\omega_{1}\right) \delta\left(\omega-\omega_{1}-\omega_{2}-\omega_{3}\right) d \omega_{2} d \omega_{3}
\end{aligned}
$$

Using equation (6.5.15), the laser response was derived as,

$$
\begin{aligned}
Y(\omega)= & \frac{1}{1 !} H_{1}\left(\omega_{1}\right) \delta\left(\omega-\omega_{1}\right)+\frac{1}{2 ! 2 \pi} H_{2}\left(\omega_{1}, \omega_{1}\right) \delta\left(\omega-2 \omega_{1}\right) \\
& +\frac{1}{3 !(2 \pi)^{2}} H_{3}\left(\omega_{1}, \omega_{1}, \omega_{1}\right) \delta\left(\omega-3 \omega_{1}\right)
\end{aligned}
$$

Using (6.5.15), (6.5.16), and (6.5.13) the general frequency domain output of the laser model for $N$ input carriers is given by,

$$
\begin{aligned}
& Y(\omega)=\sum_{n=1}^{n=3} \sum_{i=-m}^{m} \frac{1}{(2 \pi)^{n-1}} \operatorname{Re}\left\{\frac { n ! 2 ^ { - n + 1 } } { r _ { - m } ! \ldots r _ { m } ! } \left(\int_{\infty}^{\infty} \int_{\infty}^{\infty} \int_{\infty}^{\infty} H_{n}\left(\omega_{1}, \ldots, \omega_{n}\right)\right.\right. \\
&\left.\left.\times \prod_{k=1}^{N} A_{i_{k}}\left(\omega_{k}\right) d \omega_{k}\right) \exp \left(\sum_{k=1}^{n} \omega_{i_{k}}\right)\right\}
\end{aligned}
$$


Equation (6.5.17) represents the output spectrum of the laser (harmonic and intermodulation products) subjected to $\mathrm{N}$ input carriers. From (6.5.17) the harmonics and second and third order distortion levels can be predicted. Also, the complex envelope amplitude $D\left(f_{i_{1}}, \ldots, f_{i_{k}}\right)$, of the output components can be derived as,

$$
D\left(f_{i_{1}}, \ldots, f_{i_{k}}\right)=\frac{1}{(2 \pi)^{n-1}} \frac{n ! 2^{-n+1} \prod_{k=1}^{n} A_{i_{k}}}{r_{-m} ! \ldots r_{m} !} H_{n}\left(f_{i_{1}}, \ldots, f_{i_{n}}\right)
$$

in which $i_{1}, \ldots, i_{n}= \pm 1, \pm 2, \ldots, \pm N$ and the input signal amplitude for each carrier is known by $A_{i_{k}}$ or simply $=A_{i}$ and it is related to the optical modulation depth (MD). Since, the $\mathrm{MD}$ is defined as the ratio of the peak of the modulated light power to the average (DC) light power, $=A_{i}$ is given by,

$$
A_{i}=M D P_{d c} / N\left|H_{1}\left(f_{1}\right)\right|
$$

Using (6.5.16) to (6.5.19), and assuming the laser is modulated by three carriers, the harmonic and intermodulation distortion levels relative to the level of the first carrier can be derived. For example,

$$
\begin{aligned}
\left.\left.2 H D\right|_{2 f_{1}}\right|_{d B} & =20 \log \left\{\frac{1}{4} \frac{M D P_{d c}}{\left|H_{1}\left(f_{1}\right)\right|} \frac{\left|H_{2}\left(f_{1}, f_{1}\right)\right|}{\left|H_{1}\left(f_{1}\right)\right|}\right\} \\
\left.\left.2 I M D\right|_{\left(f_{1}+f_{3}\right)}\right|_{d B} & =20 \log \left\{\frac{1}{2} \frac{M D P_{d c}}{\left|H_{1}\left(f_{1}\right)\right|} \frac{\left|H_{2}\left(f_{1}, f_{3}\right)\right|}{\left|H_{1}\left(f_{3}\right)\right|}\right\} \\
\left.\left.3 I M D\right|_{\left(2 f_{1}-f_{2}\right)}\right|_{d B} & =20 \log \left\{\frac{3}{8} \frac{M D^{2} P_{d c}{ }^{2}}{\left|H_{1}\left(f_{1}\right)\right|} \frac{\left|H_{3}\left(f_{1}, f_{1},-f_{2}\right)\right|}{\left|H_{1}\left(f_{1}\right) H_{1}\left(f_{2}\right)\right|}\right\} \\
\left.\left.3 I M D\right|_{\left(2 f_{2}-f_{1}\right)}\right|_{d B} & =20 \log \left\{\frac{3}{8} \frac{M D^{2} P_{d c}{ }^{2}}{\left|H_{1}\left(f_{1}\right)\right|} \frac{\left|H_{3}\left(-f_{1}, f_{2}, f_{2}\right)\right|}{\left|H_{1}\left(f_{1}\right) H_{1}\left(f_{2}\right)\right|}\right\} \\
\left.\left.3 I M D\right|_{\left(f_{1}+f_{2}-f_{3}\right)}\right|_{d B} & =20 \log \left\{\frac{3}{4} \frac{M D^{2} P_{d c}{ }^{2}}{\left|H_{1}\left(f_{1}\right)\right|} \frac{\left|H_{3}\left(f_{1}, f_{2},-f_{3}\right)\right|}{\left|H_{1}\left(f_{2}\right) H_{1}\left(f_{3}\right)\right|}\right\}
\end{aligned}
$$

\subsubsection{Distortion evaluation after pre-distorter}

For the performance of the whole system, the pre-distorter and laser diode models are cascaded, and evaluated following the same theory as presented in Section 6.5.2.1. Recall 
Figure 6.6 and equation (6.5.16), and for simplicity consider that the input signal $I(\omega)$ is a unit impulse. The output of the pre-distorter $Y(\omega)$ is then similar to that of equation (6.5.16) after replacing $H(\omega)$ by $G(\omega) . Y(\omega)$ is then the input to the laser Volterra model. Using equation (6.5.16), the output spectrum $Y_{0}(\omega)$ of the whole system was derived as,

$$
\begin{aligned}
Y_{o}(\omega)= & \underbrace{\frac{1}{1 !} H_{1}(\omega) Y(\omega)}_{\text {o/p of the } 1^{\text {st }} \text { Volterra Operator }\left(Y_{o 1}\right)} \\
& +\underbrace{\frac{1}{2 ! 2 \pi} \int_{-\infty}^{\infty} H_{2}\left(\omega_{1}, \omega-\omega_{1}\right) Y\left(\omega_{1}\right) Y\left(\omega-\omega_{1}\right) d \omega_{1}}_{\text {o/p of the } 2^{\text {nd }} \text { Volterra Operator }\left(Y_{o 2}\right)} \\
& +\underbrace{\frac{1}{3 !(2 \pi)^{2}} \int_{-\infty}^{\infty} \int_{-\infty}^{\infty} H_{3}\left(\omega_{2}, \omega_{1}, \omega-\omega_{1}-\omega_{2}\right)}_{\text {o/p of the } 3^{\text {rd }} \text { Volterra Operator }\left(Y_{o 3}\right)}
\end{aligned}
$$

Calculating the integrals in equation 6.5 .25 and summing the outputs of the three Volterra orders leads to the overall system response. From equation 6.5 .16 , after replacing $H(\omega)$ by $G(\omega)$ in $(6.5 .25)$, the output of the $1^{3 t}, 2^{\text {nd }}$, and $3^{\text {rd }}$ order Volterra operators are calculated as follows,

- Output of the $1^{s t}$ order Volterra operators:

$$
\begin{aligned}
Y_{o 1}(f)= & 1 \times \delta\left(f-f_{o}\right)+\frac{1}{2 !} H_{1}\left(2 f_{o}\right) G_{2}\left(f_{o}, f_{0}\right) \delta\left(f-2 f_{o}\right) \\
& +\frac{1}{3 !} H_{1}\left(3 f_{o}\right) G_{3}\left(f_{o}, f_{o}, f_{o}\right) \delta\left(f-3 f_{o}\right)
\end{aligned}
$$

- Output of the $2^{\text {nd }}$ order Volterra operators: 


$$
\begin{aligned}
Y_{o 2}(f)= & \frac{1}{2 !}\left[\int _ { - \infty } ^ { \infty } H _ { 2 } ( f - f _ { 1 } , f _ { 1 } ) \left\{\frac{1}{1 !} G_{1}\left(f_{o}\right) \delta\left(f-f_{1}-f_{o}\right)\right.\right. \\
& +\frac{1}{2 !} G_{2}\left(f_{o}, f_{o}\right) \delta\left(f-f_{1}-2 f_{o}\right) \\
& \left.+\frac{1}{3 !} G_{3}\left(f_{o}, f_{o}, f_{o}\right) \delta\left(f-f_{1}-3 f_{o}\right)\right\} \\
& \left\{\frac{1}{1 !} G_{1}\left(f_{o}\right) \delta\left(f_{1}-f_{o}\right)+\frac{1}{2 !} G_{2}\left(f_{o}, f_{o}\right) \delta\left(f_{1}-2 f_{o}\right)\right. \\
& \left.\left.+\frac{1}{3 !} G_{3}\left(f_{o}, f_{o}, f_{o}\right) \delta\left(f-3 f_{o}\right)\right\} d f_{1}\right] \\
= & \frac{1}{2 !}\left\{H_{2}\left(f_{o}, f_{o}\right) G_{1}^{2}\left(f_{o}\right) \delta\left(f-2 f_{o}\right)\right. \\
& +\frac{1}{2 !} H_{2}\left(f_{o}, 2 f_{o}\right) G_{1}\left(f_{o}\right) G_{2}\left(f_{o}, f_{o}\right) \delta\left(f-3 f_{o}\right) \\
& +\frac{1}{3 !} H_{2}\left(f_{o}, 3 f_{o}\right) G_{1}\left(f_{o}\right) G_{3}\left(f_{o}, f_{o}, f_{o}\right) \delta\left(f-4 f_{o}\right) \\
& +\frac{1}{2 !} H_{2}\left(2 f_{o}, f_{o}\right) G_{2}\left(f_{o}, f_{o}\right) G_{1}\left(f_{o}\right) \delta\left(f-3 f_{o}\right) \\
& +\frac{1}{2 ! 2 !} H_{2}\left(2 f_{o}, 2 f_{o}\right) G_{2}^{2}\left(f_{o}, f_{o}\right) \delta\left(f-4 f_{o}\right) \\
& +\frac{1}{2 ! 3 !} H_{2}\left(2 f_{o}, 3 f_{o}\right) G_{2}\left(f_{o}, f_{o}\right) G_{3}\left(f_{o}, f_{o}, f_{o}\right) \delta\left(f-5 f_{o}\right) \\
& +\frac{1}{3 !} H_{2}\left(3 f_{o}, f_{o}\right) G_{3}\left(f_{o}, f_{o}, f_{o}\right) G_{1}\left(f_{o}\right) \delta\left(f-4 f_{o}\right) \\
& +\frac{1}{2 ! 3 !} H_{2}\left(3 f_{o}, 2 f_{o}\right) G_{3}\left(f_{o}, f_{o}, f_{o}\right) G_{2}\left(f_{o}, f_{o}\right) \delta\left(f-5 f_{o}\right) \\
& \left.+\frac{1}{3 ! 3 !} H_{2}\left(3 f_{o}, 3 f_{o}\right) G_{3}^{2}\left(f_{o}, f_{o}, f_{o}\right) \delta\left(f-6 f_{o}\right)\right\} \\
&
\end{aligned}
$$

The output of the $2^{\text {nd }}$ order Volterra operators is given by (6.5.29), assuming terms higher than the third order will not contribute to the output, since the nonlinear terms were expanded up to the third order, and can be neglected.

$$
\begin{aligned}
Y_{02}(f)= & \frac{1}{2 !} H_{2}\left(f_{o}, f_{o}\right) G_{1}^{2}\left(f_{o}\right) \delta\left(f-2 f_{o}\right) \\
& +\frac{1}{2 ! 2 !} H_{2}\left(f_{o}, 2 f_{o}\right) G_{1}\left(f_{o}\right) G_{2}\left(f_{o}, f_{o}\right) \delta\left(f-3 f_{o}\right) \\
& +\frac{1}{2 ! 2 !} H_{2}\left(2 f_{o}, f_{o}\right) G_{2}\left(f_{o}, f_{o}\right) G_{1}\left(f_{o}\right) \delta\left(f-3 f_{o}\right)
\end{aligned}
$$


- Output of the $3^{\text {rd }}$ order Volterra operators:

$$
\begin{aligned}
Y_{o 3}(f)= & \frac{1}{3 !}\left[\int _ { - \infty } ^ { \infty } \int _ { - \infty } ^ { \infty } H _ { 3 } ( f - f _ { 1 } - f _ { 2 } , f _ { 1 } , f _ { 2 } ) \left\{\frac{1}{1 !} G_{1}\left(f_{o}\right) \delta\left(f-f_{1}-f_{2}-f_{o}\right)\right.\right. \\
& +\frac{1}{2 !} G_{2}\left(f_{o}, f_{o}\right) \delta\left(f-f_{1}-f_{2}-2 f_{o}\right)+\frac{1}{3 !} G_{3}\left(f_{o}, f_{o}, f_{o}\right) \times \\
& \left.\delta\left(f-f_{1}-f_{2}-3 f_{o}\right)\right\}\left\{\frac{1}{1 !} G_{1}\left(f_{o}\right) \delta\left(f_{1}-f_{o}\right)+\frac{1}{2 !} G_{2}\left(f_{o}, f_{o}\right) \delta\left(f_{1}-2 f_{o}\right)\right. \\
& \left.+\frac{1}{3 !} G_{3}\left(f_{o}, f_{o}, f_{o}\right) \delta\left(f-3 f_{o}\right)\right\}\left\{\frac{1}{1 !} G_{1}\left(f_{o}\right) \delta\left(f_{2}-f_{o}\right)\right. \\
+ & \left.\left.\frac{1}{2 !} G_{2}\left(f_{o}, f_{o}\right) \delta\left(f_{2}-2 f_{o}\right)+\frac{1}{3 !} G_{3}\left(f_{o}, f_{o}, f_{o}\right) \delta\left(f_{2}-3 f_{o}\right)\right\} d f_{1} d f_{2}\right] \quad(6.5 .30) \\
= & \frac{1}{3 !}\left\{H_{3}\left(f_{o}, f_{o}, f_{o}\right) G_{1}^{3}\left(f_{0}\right) \delta\left(f-3 f_{o}\right)\right. \\
& +\frac{1}{2 !} H_{3}\left(f_{o}, f_{o}, 2 f_{o}\right) G_{1}^{2}\left(f_{o}\right) G_{2}\left(f_{o}, f_{o}\right) \delta\left(f-4 f_{o}\right) \\
& +\frac{1}{3 !} H_{3}\left(f_{o}, f_{o}, 3 f_{o}\right) G_{1}^{2}\left(f_{o}\right) G_{3}\left(f_{o}, f_{o}, f_{o}\right) \delta\left(f-5 f_{o}\right) \\
& \cdot \\
& \cdot+\frac{1}{3 ! 3 !} H_{3}\left(3 f_{o}, 3 f_{o}, f_{o}\right) G_{1}\left(f_{o}\right) G_{3}^{2}\left(f_{o}, f_{o}, f_{o}\right) \delta\left(f-7 f_{o}\right) \\
& +\frac{1}{3 ! 3 ! 2 !} H_{3}\left(3 f_{o}, 3 f_{o}, 2 f_{o}\right) G_{2}\left(f_{o}, f_{o}\right) G_{3}^{2}\left(f_{o}, f_{o}, f_{o}\right) \delta\left(f-8 f_{o}\right) \\
& \left.+\frac{1}{3 ! 3 ! 3 !} H_{3}\left(3 f_{o}, 3 f_{o}, 3 f_{o}\right) G_{3}^{3}\left(f_{o}, f_{o}, f_{o}\right) \delta\left(f-9 f_{o}\right)\right\} \\
&
\end{aligned}
$$

The output of the $3^{\text {rd }}$ order Volterra operators is given by (6.5.32), assuming terms higher than the third order will not contribute to the output, since the nonlinear terms were expanded up to the third order,

$$
Y_{o 3}(f)=\frac{1}{3 !} H_{3}\left(f_{o}, f_{o}, f_{o}\right) G_{1}^{3}\left(f_{o}\right) \delta\left(f-3 f_{o}\right)
$$


Using equations $(6.5 .26),(6.5 .29)$, and $(6.5 .32)$, the total output of the whole system is given by,

$$
\begin{aligned}
Y_{o}(f)= & 1 \times \delta\left(f-f_{o}\right) \\
+ & +\frac{1}{2 !}\left[H_{1}\left(2 f_{o}\right) G_{2}\left(f_{o}, f_{o}\right)+H_{2}\left(f_{o}, f_{o}\right) G_{1}^{2}\left(f_{o}\right)\right] \delta\left(f-2 f_{o}\right) \\
+ & \frac{1}{3 !}\left[H_{1}\left(3 f_{0}\right) G_{3}\left(f_{o}, f_{o}, f_{0}\right)+H_{2}\left(f_{o}, 2 f_{o}\right) G_{1}\left(f_{o}\right) G_{2}\left(f_{o}, f_{o}\right)\right. \\
& +H_{2}\left(2 f_{o}, f_{o}\right) G_{2}\left(f_{o}, f_{o}\right) G_{1}\left(f_{o}\right) \\
& \left.+H_{3}\left(f_{o}, f_{o}, f_{o}\right) G_{1}^{3}\left(f_{o}\right)\right] \delta\left(f-3 f_{o}\right)
\end{aligned}
$$

The overall output of the system, assuming the input signal to the pre-distorter consists of $N$ carriers, was derived by using equation (6.5.25) after replacing the unit impulse by the sum of the three carriers as given by (6.5.13) and (6.5.19). Examples of the harmonic and intermodulation distortion levels in $\mathrm{dB}$ (of the laser cascaded with the pre-distorter) relative to the level of the first carrier and assuming the input is the sum of three independent carriers of frequencies $f_{1}, f_{2}$, and $f_{3}$ are,

$$
\begin{aligned}
\left.2 H D\right|_{2 f_{1}} ^{W P D}=20 \log \{ & \left\{\frac{1}{2} M D\left(I_{b}-I_{t h}\right) P_{d c} \mid H_{1}\left(2 f_{1}\right) G_{2}\left(f_{1}, f_{1}\right)\right. \\
& \left.+H_{2}\left(f_{1}, f_{1}\right)\left(G_{1}\left(f_{1}\right)\right)^{2} \mid\right\} \\
\left.2 I M D\right|_{\left(f_{1}+f_{3}\right)} ^{W P D}=20 \log \left\{\frac{1}{2} M D\left(I_{b}-I_{t h}\right) P_{d c} \mid H_{1}\left(f_{1}+f_{3}\right) G_{2}\left(f_{1}, f_{3}\right)\right. & \left.+H_{2}\left(f_{1},-f_{3}\right) G_{1}\left(f_{1}\right) G_{1}\left(-f_{3}\right) \mid\right\} \\
\left.3 I M D\right|_{\left(2 f_{1}-f_{2}\right)} ^{W P D} 20 \log \left\{\frac{3}{4} M D^{2}\left(I_{b}-I_{t h}\right)^{2} P_{d c}^{2} \mid H_{1}\left(f_{1}+f_{1}-f_{2}\right) G_{3}\left(f_{1}, f_{1},-f_{2}\right)\right. & +H_{2}\left(f_{1},\left(f_{1}-f_{2}\right)\right) G_{1}\left(f_{1}\right) G_{2}\left(f_{1},\left(f_{1}-f_{2}\right)\right) \\
& +H_{2}\left(\left(f_{1}-f_{2}\right),-f_{2}\right) G_{2}\left(\left(f_{1}-f_{2}\right),-f_{2}\right) G_{1}\left(f_{1}-f_{2}\right) \\
& \left.+H_{3}\left(f_{1}, f_{1},-f_{2}\right)\left(G_{1}\left(f_{1}\right)\right)^{2} G_{1}\left(-f_{2}\right) \mid\right\}
\end{aligned}
$$




$$
\begin{aligned}
\left.3 M M\right|_{\left(f_{1}+f_{2}-f_{3}\right)} ^{W P D}=20 \log & \left\{\frac{3}{4} M D^{2}\left(I_{b}-I_{t h}\right)^{2} P_{d c}^{2} \mid H_{1}\left(f_{1}+f_{2}-f_{3}\right) G_{3}\left(f_{1}, f_{2},-f_{3}\right)\right. \\
& +H_{2}\left(f_{1},\left(f_{2}-f_{3}\right)\right) G_{1}\left(f_{1}\right) G_{2}\left(f_{1},\left(f_{2}-f_{3}\right)\right) \\
& +H_{2}\left(\left(f_{1}+f_{2}\right),-f_{3}\right) G_{2}\left(\left(f_{1}+f_{2}\right),-f_{3}\right) G_{1}\left(f_{1}-f_{2}\right) \\
& \left.+H_{3}\left(f_{1}, f_{2},-f_{3}\right) G_{1}\left(f_{1}\right) G_{1}\left(f_{2}\right) G_{1}\left(-f_{3}\right) \mid\right\}
\end{aligned}
$$

\subsubsection{Pre-distorter Implementation and Testing}

In the previous sections, we proposed and demonstrated a linearization scheme that in principle can compensate the laser distortion. The system illustrated in Figure 6.6 was implemented in software. In order to assess the distortion levels of a semiconductor laser, the system was tested by a series of single, two and three tones at different bias levels over a wide range of carrier frequencies. Distortion levels as predicted by the pre-distorter based on the MGF, compared with that predicted by the proposed pre-distorter based on the CGF, are presented. Moreover, the distortion levels of two different semiconductor lasers (1.3 $\mu \mathrm{m}$ InGaAsP-InP Ortel 1510B and Fujitsu FLD3F7CZ) are predicted using realistic parameters such as those shown in Table 6.1 for Ortel 1510B [181] and Fujitsu FLD3F7CZ.

Predicted distortion levels for Ortel and Fujitsu lasers at $I_{b}=1.67 I_{t h}$, and modulation depth $M D=0.14$ and the corresponding enhancements using the software implemented CGF-based and MGF-based pre-distorter are illustrated in Figures 6.7 - 6.14.

A single tone input was swept in frequency up to $10 \mathrm{GHz}$ and used to test the ability of the proposed pre-distorter to reduce the second harmonic distortion of the laser. The simulation results of the predicted second harmonic distortion with and without using the pre-distorter for Ortel and Fujitsu lasers are presented in Figures 6.7 and 6.8. The simulations were repeated using CGF-based and MGF-based pre-distorter.

From Figure 6.7, the distortion levels and their enhancement ratios are over estimated using 
Table 6.1: Parameters values for 1.3 $\mu \mathrm{m}$ InGaAsP/InP semiconductor Ortel $1510 \mathrm{~B}$ and Fujitsu FLD3F7CZ laser diodes

\begin{tabular}{||l|l|l|l||}
\hline \hline \multicolumn{1}{|c|}{ Parameter } & $\begin{array}{c}\text { Symbol, } \\
\text { Unit }\end{array}$ & $\begin{array}{c}\text { IVijitsu } \\
\text { FLD3F7CZ }\end{array}$ & \multicolumn{1}{|c||}{$\begin{array}{c}\text { Ortel } \\
\text { 1510B }\end{array}$} \\
\hline \hline Operating wave length & $\lambda, \mu m$ & 1.3 & 1.3 \\
\hline Active region volume & $V_{c}, \mu m^{3}$ & $25 \times 10^{-18}$ & $8 \times 10^{-17}$ \\
\hline Transparency carrier density & $\mathbb{C}_{t r o}, m^{-3}$ & $1 \times 10^{24}$ & $1 \times 10^{24}$ \\
\hline Mode confinement factor & $\Gamma$ & 0.165 & 0.35 \\
\hline Gain coefficient & $\mathrm{g}, m^{2}$ & $3.83 \times 10^{-12}$ & $3.3 \times 10^{-12}$ \\
\hline Spontaneous emission factor & $\beta_{s p}$ & $5 \times 10^{-5}$ & $10^{-3}$ \\
Photon life time & $\tau_{p h}, \mathrm{~s}$ & $1 \times 10^{-12}$ & $1 \times 10^{-9}$ \\
\hline Electrons life time & $\tau_{e}, \mathrm{~s}$ & $1 \times 10^{-12}$ & $1.6 \times 10^{-9}$ \\
\hline Gain compression & $\epsilon, m^{-3}$ & $2.3 \times 10^{-24}$ & $3.2 \times 10^{-23}$ \\
\hline \hline
\end{tabular}

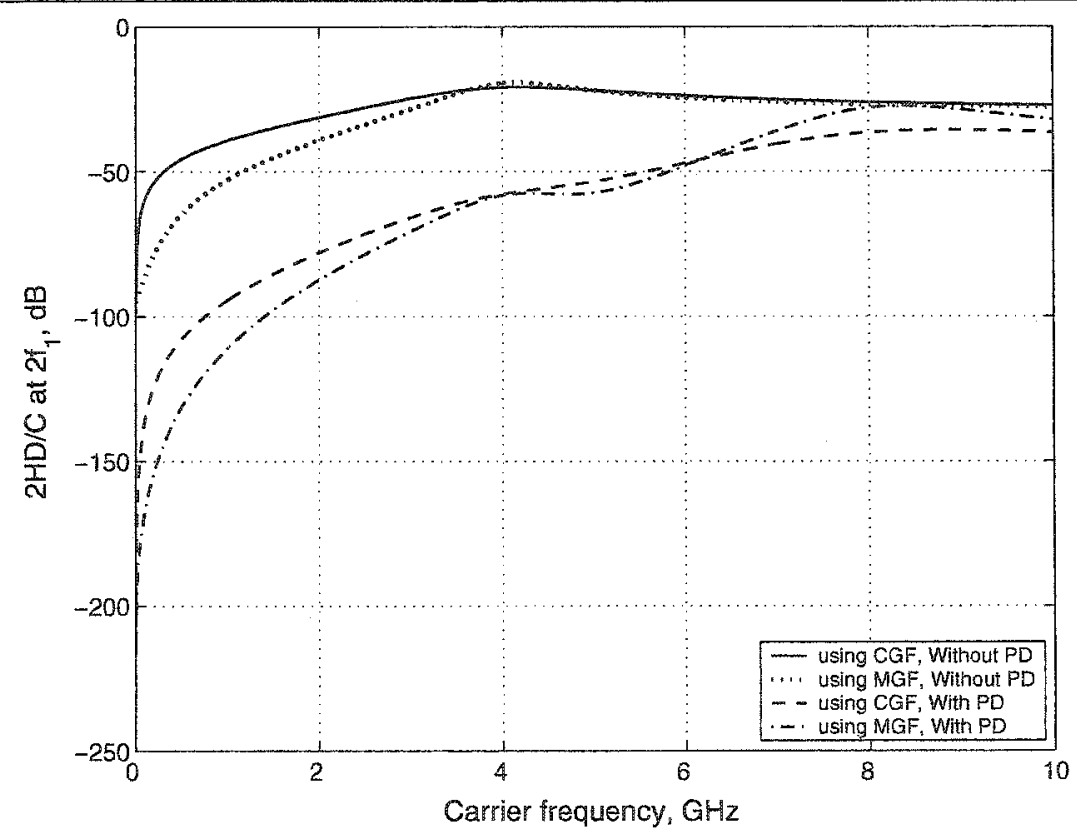

Figure 6.7: Simulation results for second harmonic distortion relative to carrier for Ortel $1510 \mathrm{~B}$ laser, with and without using the proposed pre-distorter based on CGF and MGF at $I_{b}=1.67 I_{\text {th }}$ and $\mathrm{MD}=0.14$.

CGF-based models while the MGF-based models give more accurate results compared with the measurement results. A significant difference can be observed from Figures 6.7 and 6.8 between the wide band reduction ability of the models using Ortel laser parameters [181] 


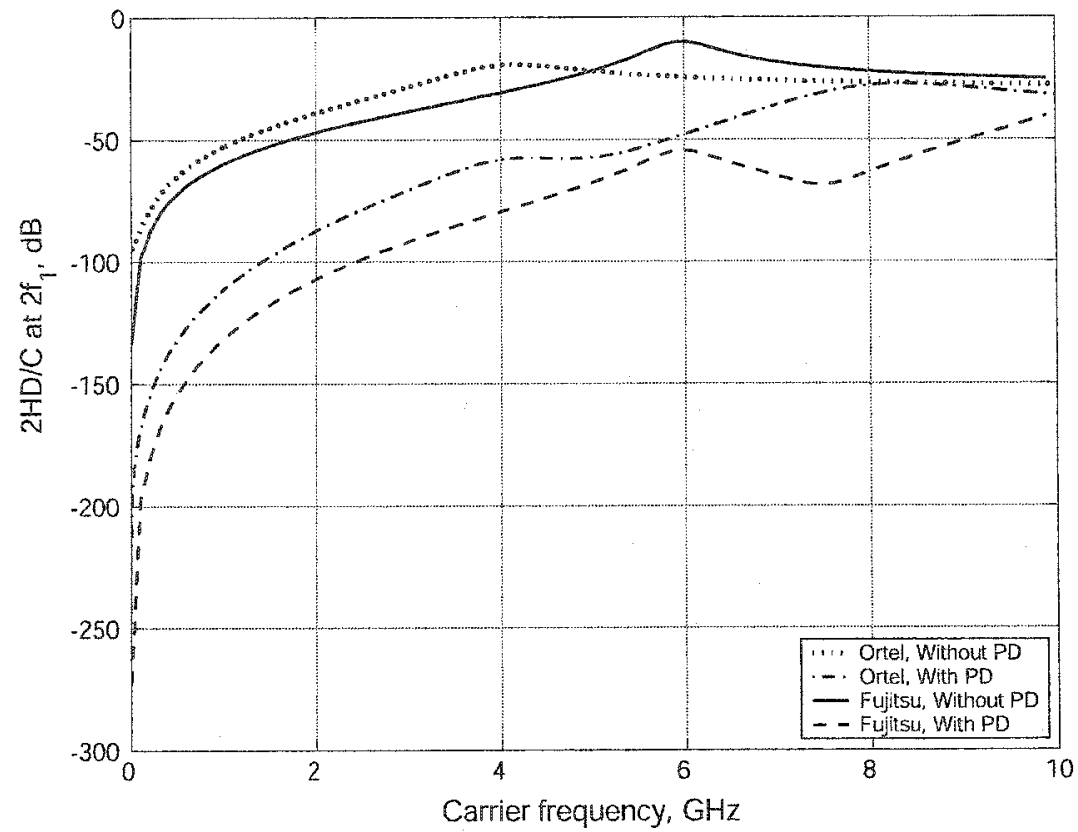

Figure 6.8: Simulation results for second harmonic distortion relative to carrier for Fujitsu FLD3F7CZ and Ortel $1510 \mathrm{~B}$ lasers, with and without using the proposed predistorter at $I_{b}=1.67 I_{\text {th }}$ and $\mathrm{MD}=0.14[14]$.

and using Fujitsu laser extracted parameters presented in Appendix G [45]. We have found that changes in the device parameters can significantly change the observed difference in the reduction characteristics and bandwidth. Also, we investigated the reason for this difference and found that it is mainly due to inconsistencies between some of the extracted parameters for the Ortel laser such as the gain coefficient, gain saturation and cavity volume. An improved extraction routine presented in in Appendix G [45] gave reasonable results. Simulation results shown in Figures 6.7 and 6.8 indicate that an average second harmonic distortion reduction of $40 \mathrm{~dB}$ over a bandwidth of approximately $6 \mathrm{GHz}$ for the Ortel laser and $55 \mathrm{~dB}$ over a bandwidth of approximately $10 \mathrm{GHz}$ for the Fujitsu laser is possible.

Figures 6.9 and 6.10 illustrate the simulation results of the second order intermodulation distortion of type $f_{1}+f_{2}$ and the corresponding enhancements for Ortel and Fujitsu lasers at $l_{b}=1.67 I$ th, and $M D=0.14$ by varying the central frequency $f_{c}$ of two tones $f_{1}$ and 
$f_{2}$ separated by $\Delta f=10 \mathrm{MHz}, f_{1}=f_{c}-\Delta f$ and $f_{2}=f_{c}+\Delta f$.

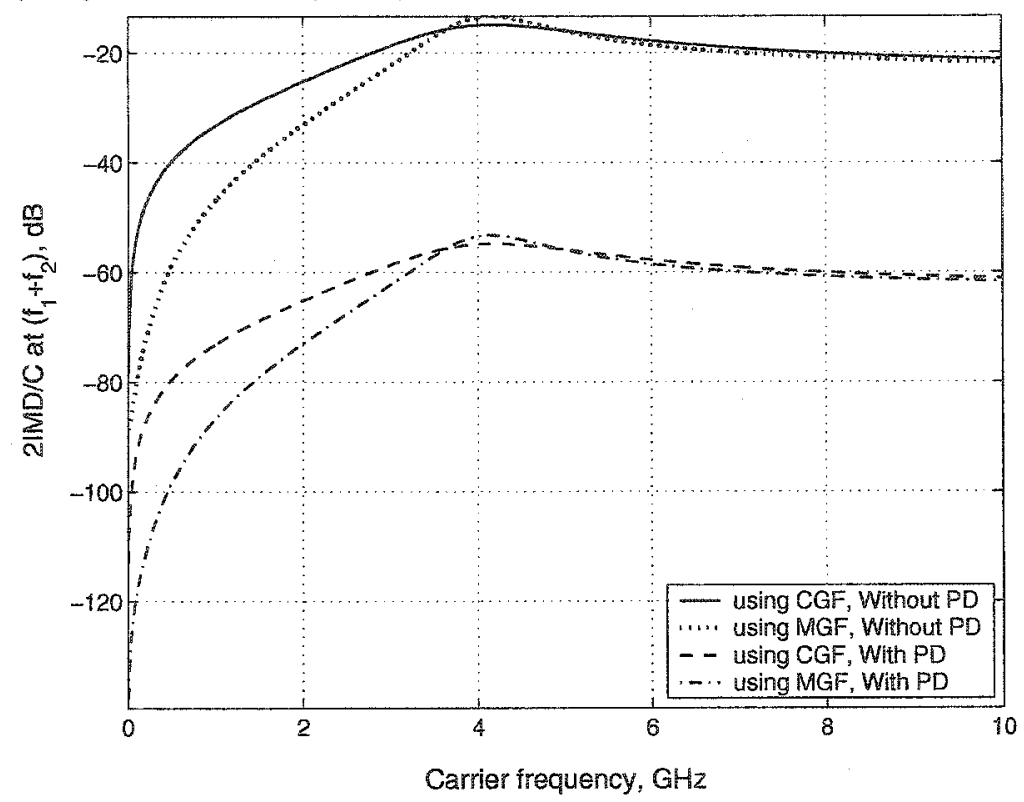

Figure 6.9: Simulated second order intermodulation distortion (2IMD) for Ortel 1510B laser, with and without using the proposed pre-distorter based on CGF and MGF at $I_{b}=1.67 I_{t h}, \mathrm{MD}=0.14, \Delta f=10 \mathrm{MHz}[9]$.

Figure 6.9 illustrates the simulation results using CGF-based and MGF-based models. As can be seen from Figure 6.10, the second order intermodulation distortion is reduced by an average of $40 \mathrm{~dB}$ for the Ortel laser and of $45 \mathrm{~dB}$ for the Fujitsu laser over a $10 \mathrm{GHz}$ frequency range.

Simulation results for the third order intermodulation distortion of type $2 f_{1}-f_{2}$ and its corresponding enhancement for the Ortel and Fujitsu lasers at $I_{b}=1.67$ Ith obtained by sweeping the center frequency $f_{c}$ of two tones $f_{1}$ and $f_{2}$ separated by $\Delta f=10 \mathrm{MHz}$ are shown in Figures 6.11 and 6.12 .

From Figure 6.11, simulations using CGF-based and MGF-based models give approximately equal third order intermodulation distortion results for the Ortel laser in the frequency range up to $4 \mathrm{GHz}$. From Figure 6.12 , the results of the third order intermodulation distortion reduction for the Ortel laser show a dip at frequencies near the relaxation oscil- 


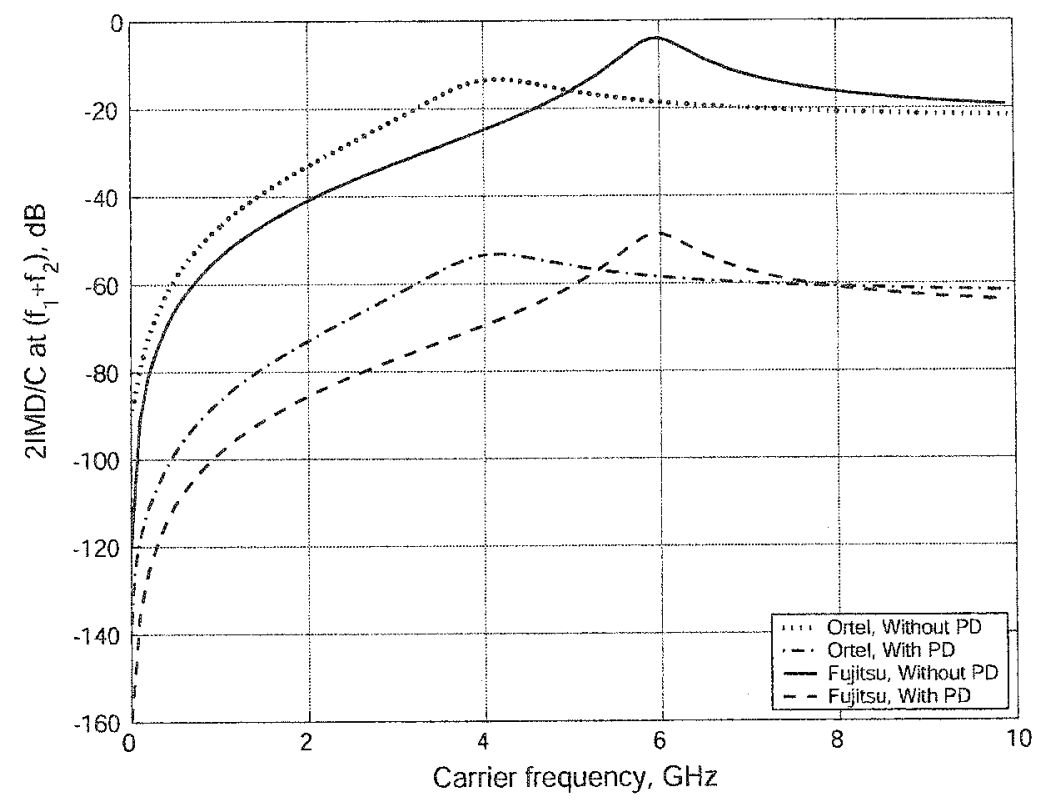

Figure 6.10: Simulation results for second order intermodulation distortion (2IMD) relative to carrier for Fujitsu FLD3F7CZ and Ortel $1510 B$ lasers, with and without using the proposed pre-distorter at $I_{b}=1.67 I_{t h}, \mathrm{MD}=0.14, \Delta f=10 \mathrm{MHz}[14]$.

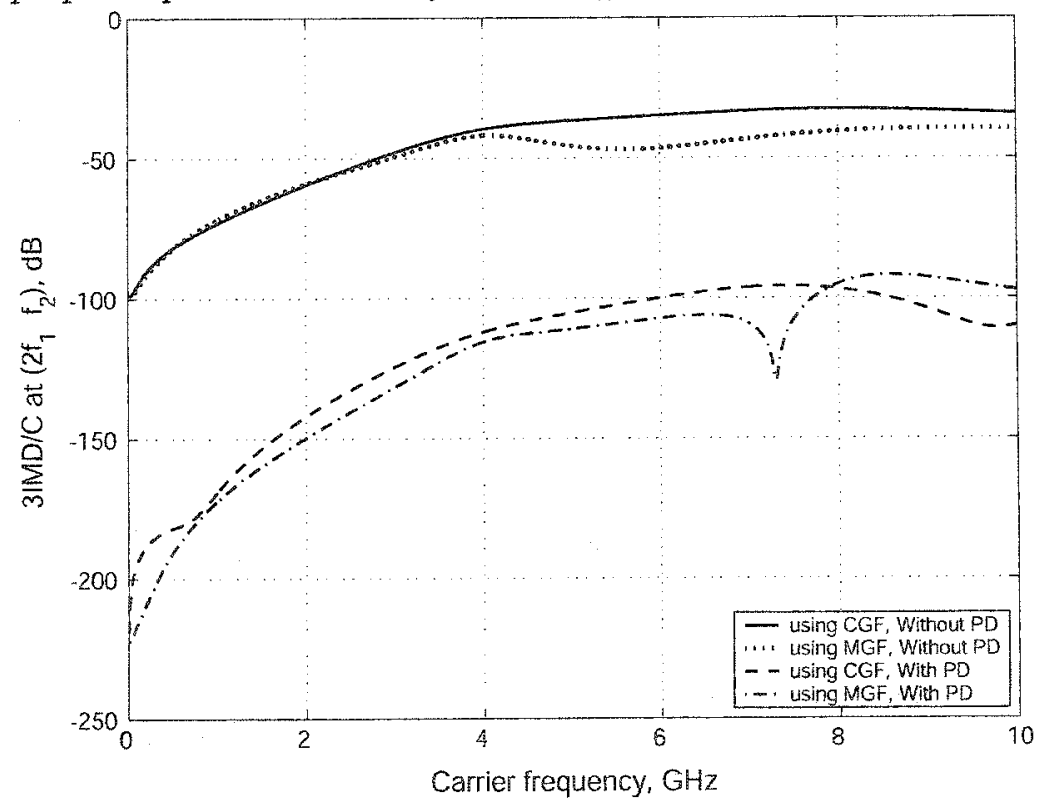

Figure 6.11: Simulation results for lower $\left(2 f_{1}-f_{2}\right)$ third order intermodulation distortion for Ortel $1510 \mathrm{~B}$ laser, with and without using the proposed pre-distorter at $I_{b}=$ $1.67 I_{\text {th }}, \mathrm{MD}=0.14, \Delta f=10 \mathrm{MHz}$. 


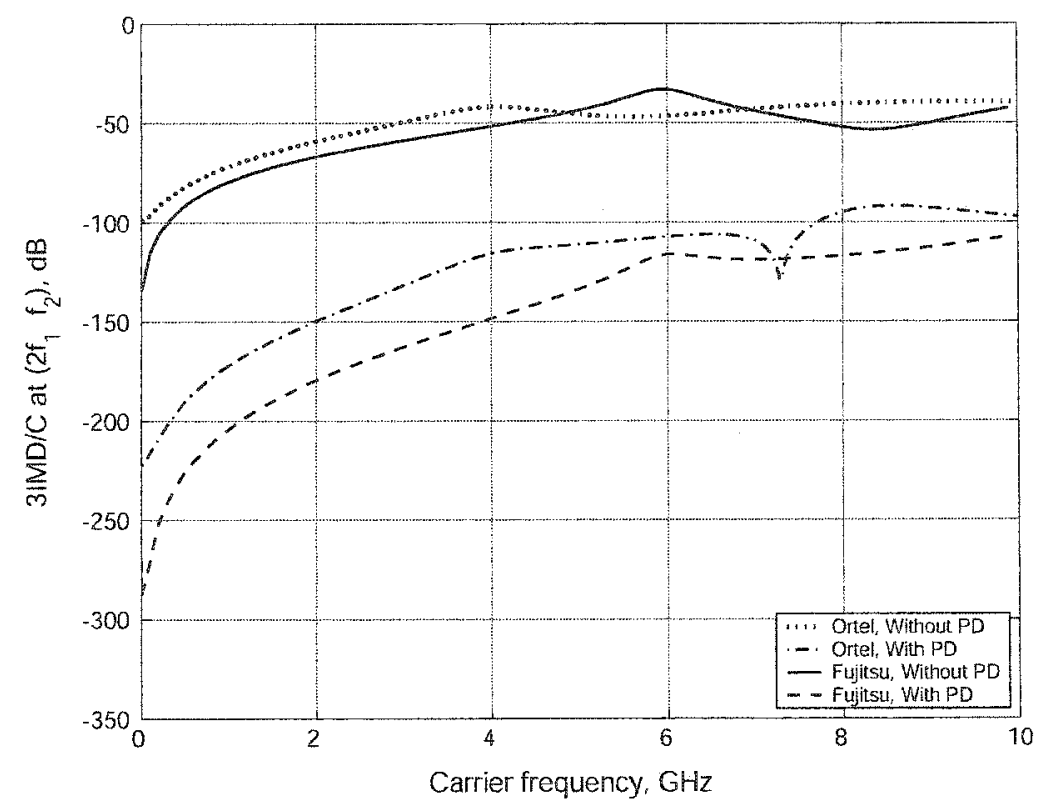

Figure 6.12: Simulation results for lower $\left(2 f_{1}-f_{2}\right)$ third order intermodulation distortion relative to carrier for Fujitsu FLD3F7CZ and Ortel 1510B lasers, with and without

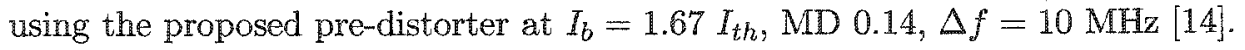

lation frequency while it did not appear in the simulation results for the Fujitsu laser. The observed dip did not appear for higher bias levels. Differences in the sensitivities to the extracted and measured laser parameters between the forward and the inverse model cause the dip to appear. Results shown in Figure 6.12 indicate that the two-tone third order intermodulation distortion at $2 f_{1}-f_{2}$ improved by an average of $70 \mathrm{~dB}$ versus $95 \mathrm{~dB}$ for the Ortel and Fujitsu lasers respectively over a $10 \mathrm{GHz}$ frequency range (ideal improvement in software).

Simulation results of the third order intermodulation distortion of type $f_{1}+f_{2}-f_{3}$ and its corresponding enhancements obtained by varying the center frequency $f_{c}$ of three-tones $f_{1}, f_{2}$, and $f_{3}$ separated by $\Delta f=10 \mathrm{MHz}, f_{2}=f_{c}, f_{1}=f_{c}-\Delta f$ and $f_{3}=f_{c}+\Delta f$ for the Ortel and Fujitsu lasers at the same operating conditions is shown in Figures 6.13 and 6.14 . Simulation results presented in Figure 6.14 show that the three-tone third-order intermod- 
ulation distortion at $f_{1}+f_{2}-f_{3}$ improved by an average of $75 \mathrm{~dB}$ versus $100 \mathrm{~dB}$ for the Ortel and Fujitsu lasers respectively over a $10 \mathrm{GHz}$ frequency range. 


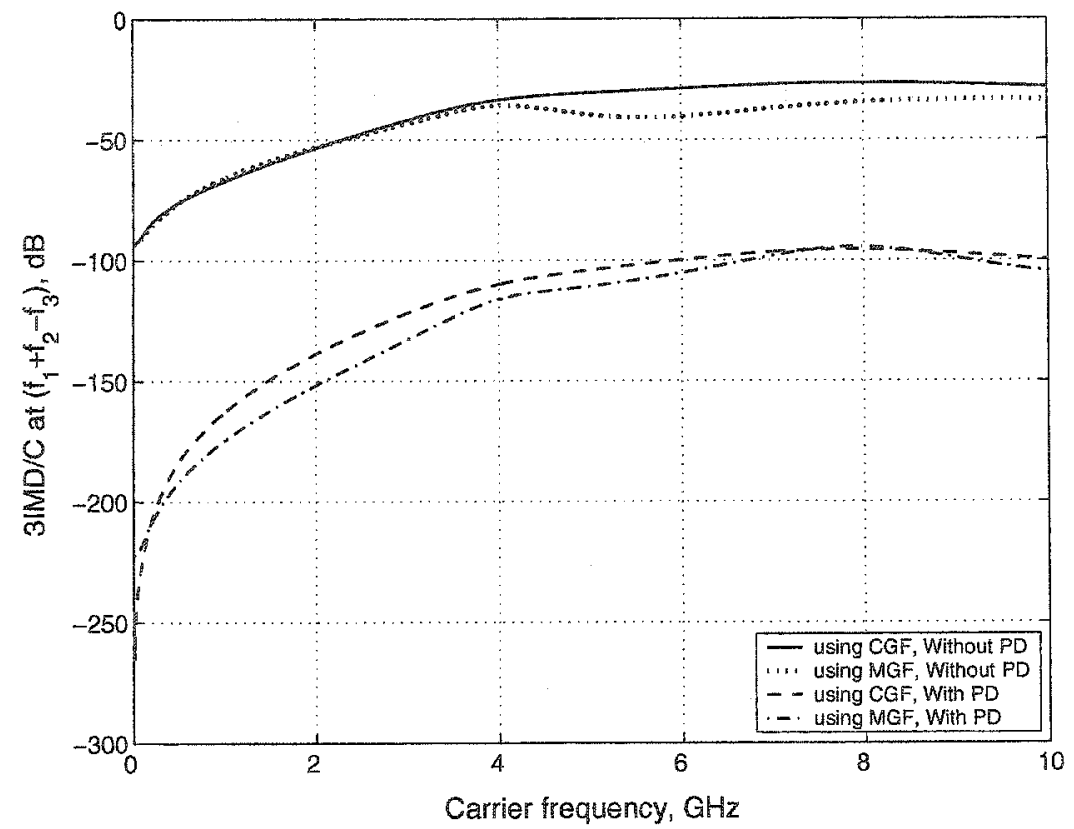

Figure 6.13: Simulation results for three tones third order intermodulation distortion relative to carrier for Ortel $1510 \mathrm{~B}$ laser, with and without using the proposed pre-distorter at $I_{b}=1.67 I_{\text {th }}, \mathrm{MD}=0.14, \Delta f=10 \mathrm{MHz}[9]$.

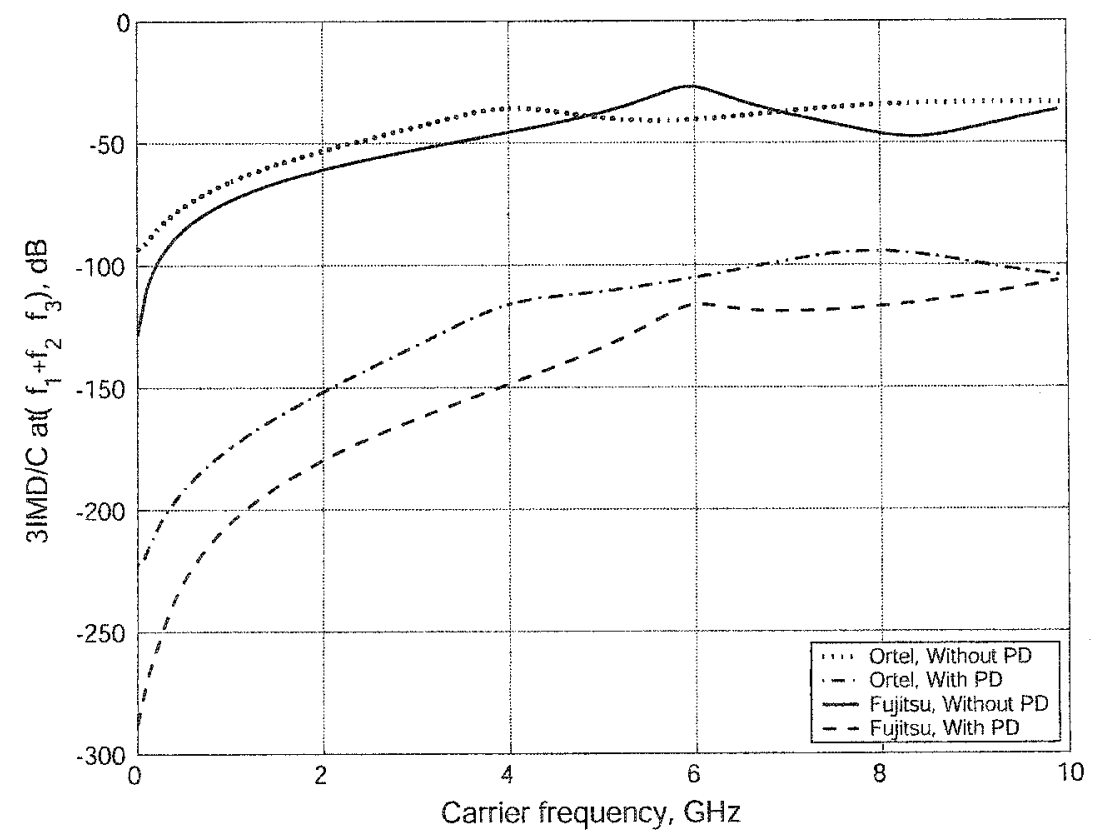

Figure 6.14: Simulation results for three tones third order intermodulation distortion relative to carrier for Fujitsu FLD3F7CZ and Ortel $1510 \mathrm{~B}$ lasers, with and without using the proposed pre-distorter at $I_{b}=1.67 I_{t h}, \mathrm{MD}=0.14, \Delta f=10 \mathrm{MHz}[14]$. 
Performance evaluation and capability tests of the proposed pre-distorter were extended by performing simulations under different bias levels and several changes of the laser parameters. Intermodulation distortion simulation results with and without using the MGF-based pre-distorter were acquired at bias levels $I_{b}=1.25,1.75$, and $2.5 I_{t h}$ and constant modulation index, 0.14, for the Ortel laser are illustrated in Figures $6.15-6.18$ while the results at two bias levels $I_{b}=1.75$, and 2.5 $I_{\text {in }}$ for the Fujitsu laser are shown in Figures 6.19-6.22. As shown in Figure 6.17, the frequency of the null in the third order intermodulation curve increases with bias current. Based on the simulation results presented in Figs. 6.17 and 6.21 the pre-distorter improvement ability is affected by the change of the bias current; this is because of the indirect relation between the coefficients of each order and the laser operating conditions. Therefore, a suitable compromise between the bias current and the reduction level for each application is recommended. The best performance observed was for bias levels in the range of 2 to 3 times the threshold level.

The simulation results are sensitive to the choice of the laser parameters and bias current. Third order distortion levels due to three-tone intermodulation products at $f_{1}+f_{2}-f_{3}$ are higher than those produced by the two-tone intermodulation products by about $6 \mathrm{dBc}$. The pre-distorter designed using the more accurate model proposed in Chapter 3 improves the distortion levels, particularly the higher order distortion levels, more than the conventional gain based pre-distorter. The presented results show that the proposed pre-distorter can significantly improve the dynamic performance of analog optical communication for cellular, CATV, and satellite systems. 


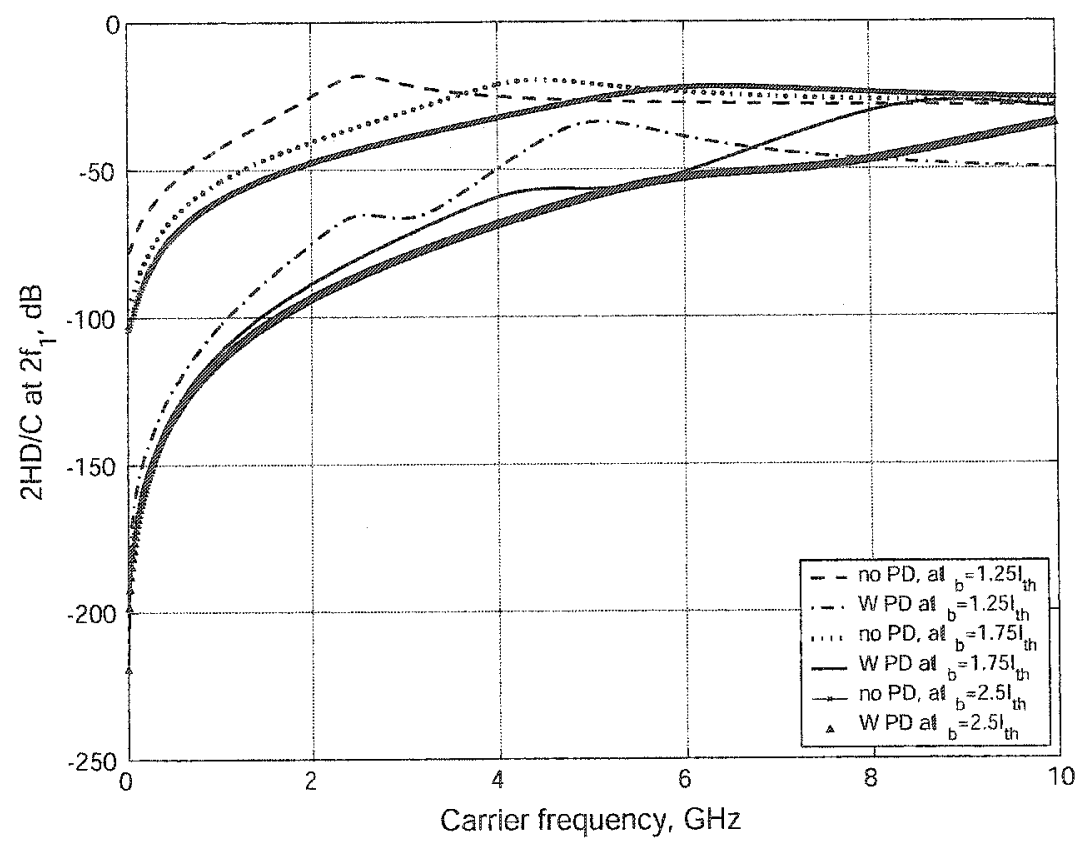

Figure 6.15: Simulation results for second harmonic distortion relative to carrier for Ortel $1510 \mathrm{~B}$ laser, with and without using the proposed pre-distorter at bias levels $I_{b}=1.25$, 1.75 , and $2.5 I_{\text {th }}$ and $\mathrm{MD}=0.14$.

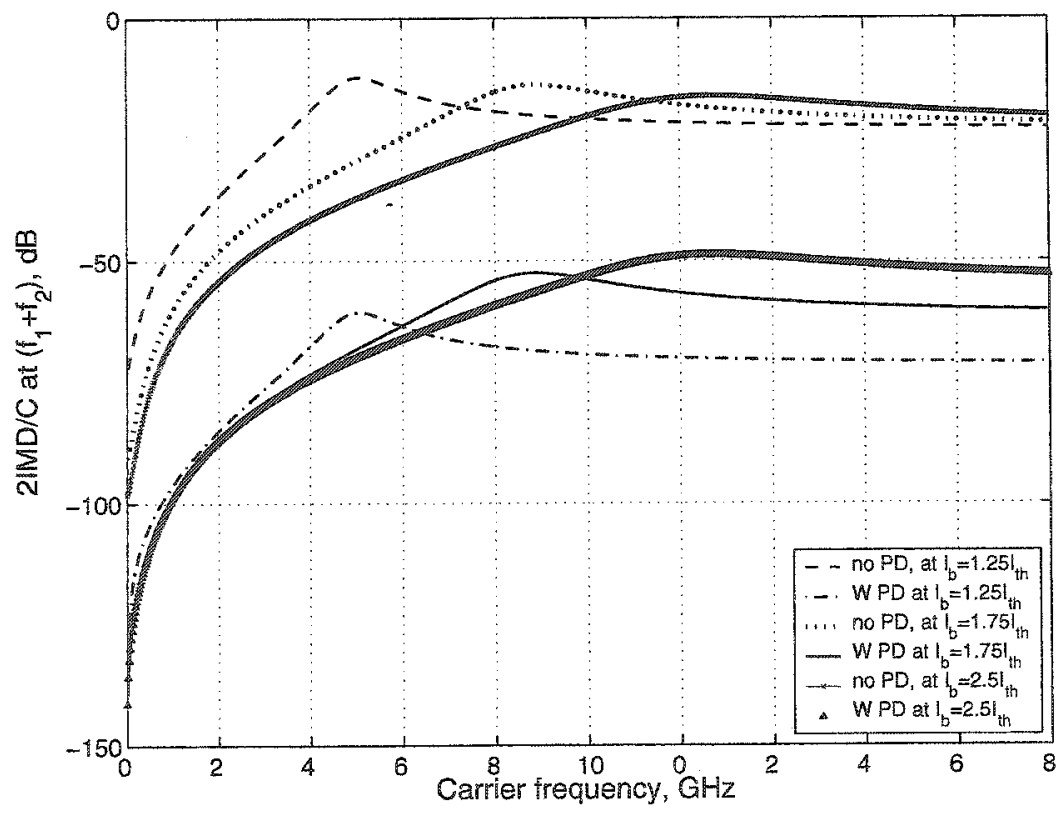

Figure 6.16: Simulation results for second order intermodulation distortion (2IMD) relative to carrier for Ortel $1510 \mathrm{~B}$ laser, with and without using the proposed pre-distorter at bias levels $I_{b}=1.25,1.75$, and $2.5 I_{t h}, \mathrm{MD}=0.14$ and $\Delta f=10 \mathrm{MHz}$. 


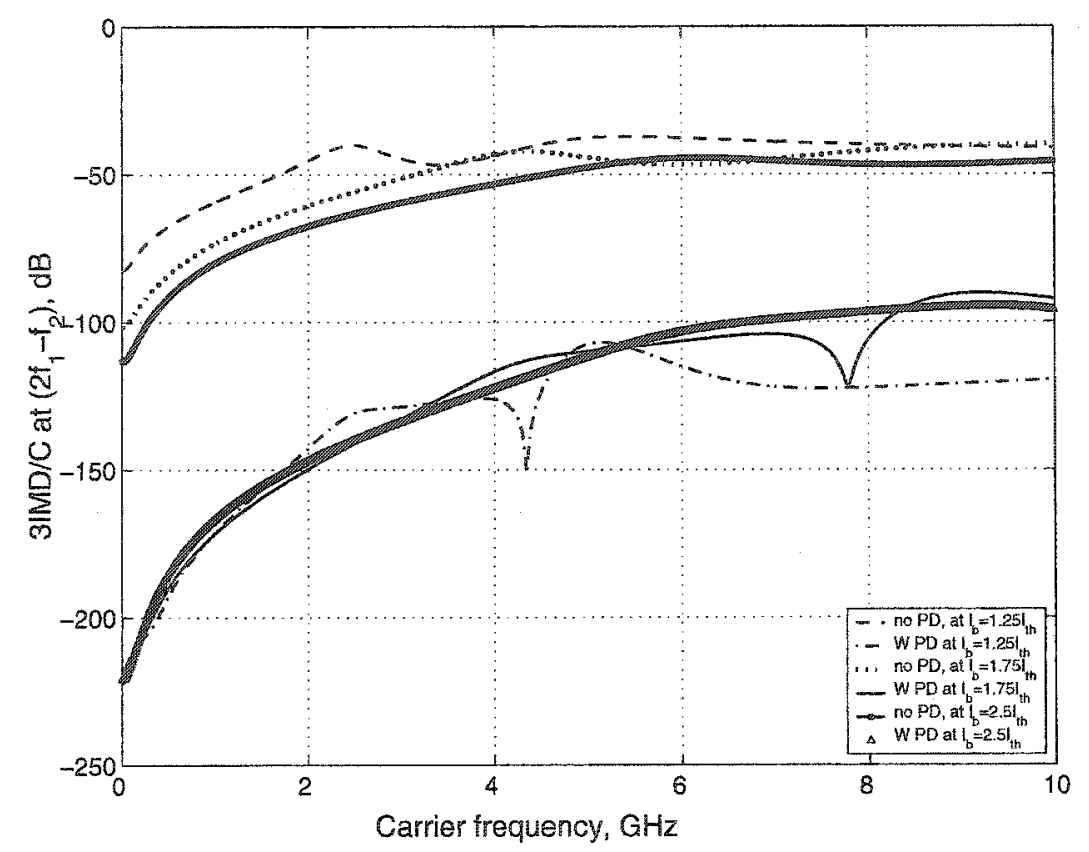

Figure 6.17: Simulation results for lower $\left(2 f_{1}-f_{2}\right)$ third order intermodulation distortion for Ortel 1510B laser, without and with using the proposed pre-distorter at bias levels $I_{b}=1.25,1.75$, and $2.5 I_{t h}, \mathrm{MD}=0.14$ and $\Delta f=10 \mathrm{MHz}[14]$.

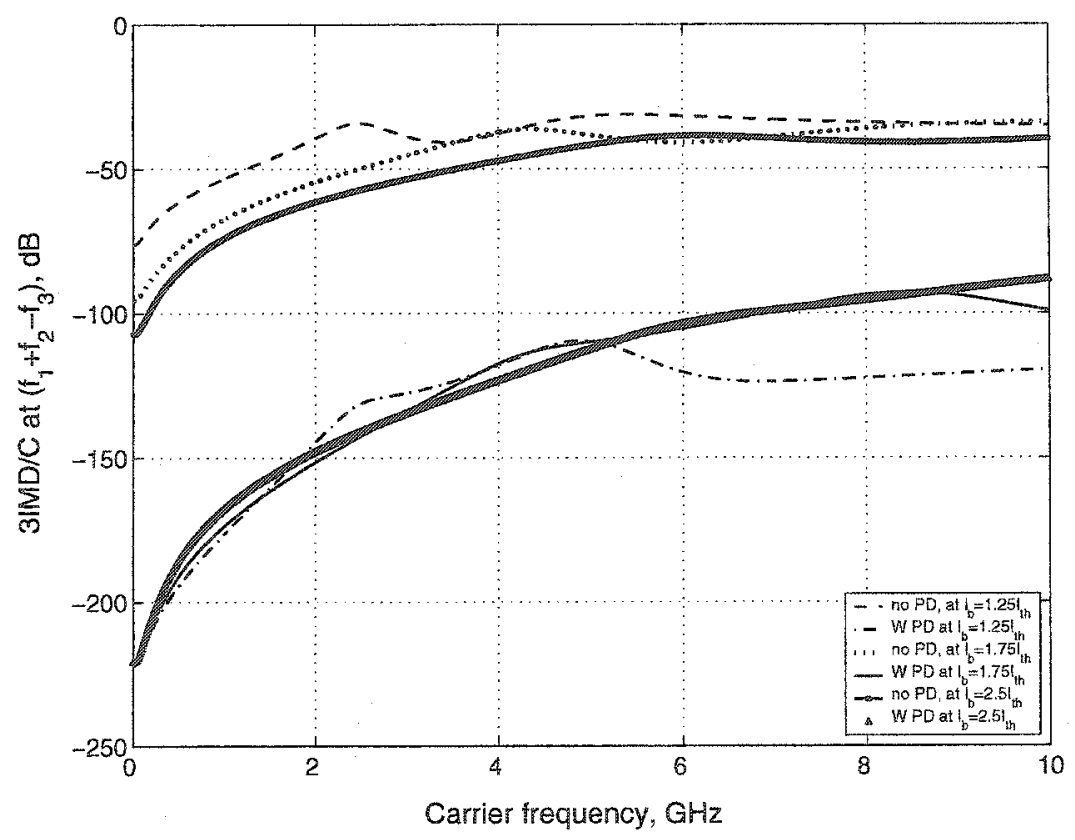

Figure 6.18: Simulation results for three tones third order intermodulation distortion relative to carrier for Ortel $1510 \mathrm{~B}$ lasers, with and without using the proposed pre-distorter at bias levels $I_{b}=1.25,1.75$, and $2.5 I_{t h}, \mathrm{MD}=0.14$ and $\Delta f=10 \mathrm{MHz}$. 


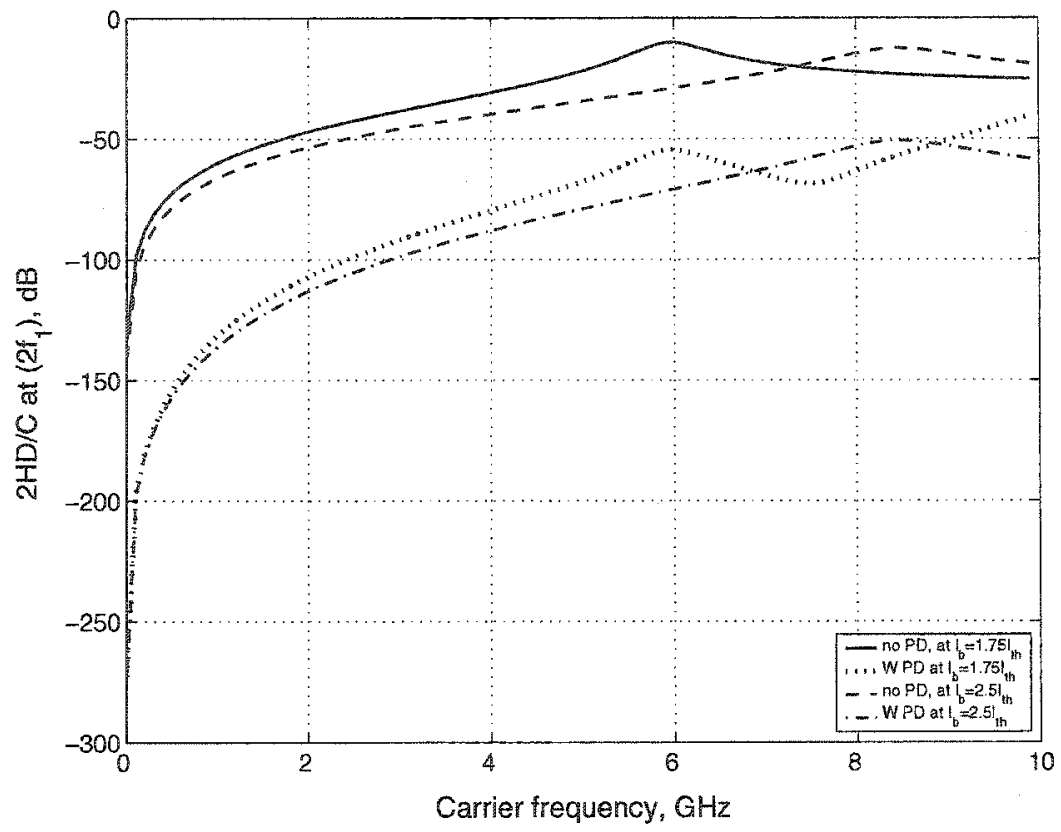

Figure 6.19: Simulation results for second harmonic distortion relative to carrier for Fujitsu FLD3F7CZ laser, with and without using the proposed pre-distorter at bias levels $I_{b}=1.75$ and $2.5 I_{t h}$ and $M D=0.14$.

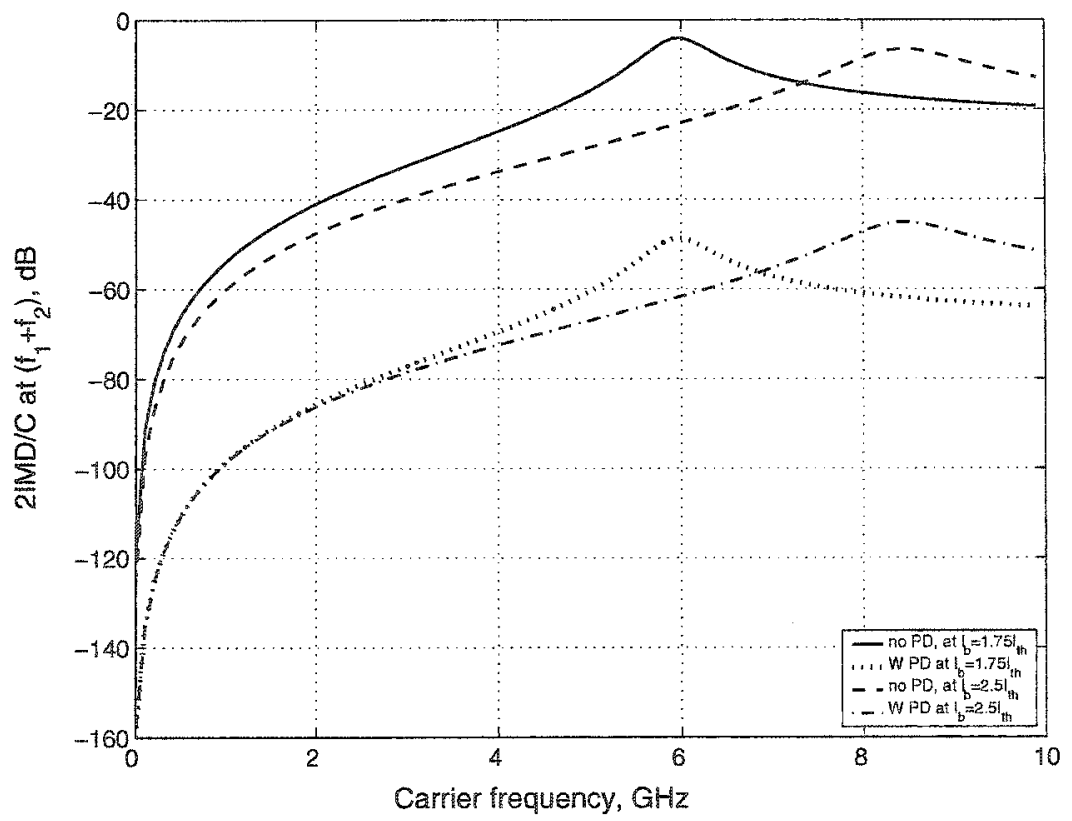

Figure 6.20: Simulation results for second order intermodulation distortion (2IMD) relative to carrier for Fujitsu FLD3F7CZ laser, with and without using the proposed predistorter at bias levels $I_{b}=1.75$ and $2.5 I_{t h}, \mathrm{MD0} .14$ and $\Delta f=10 \mathrm{MHz}$. 


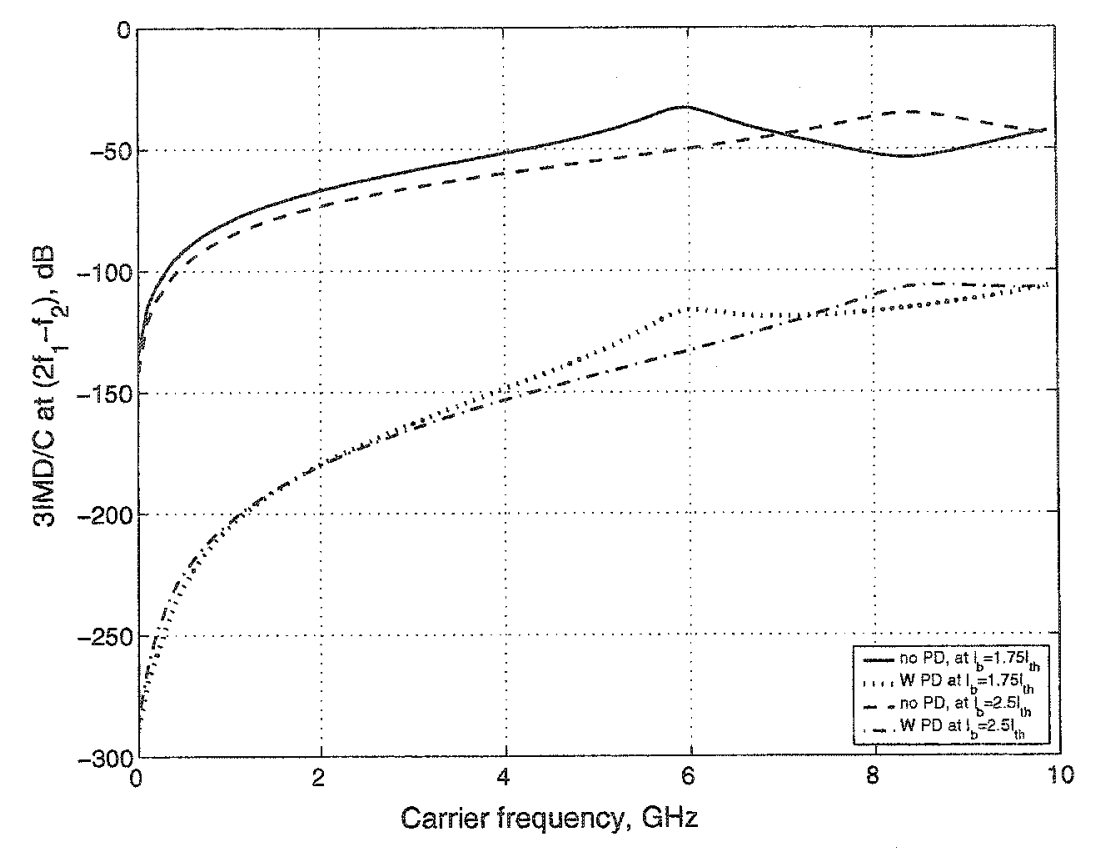

Figure 6.21: Simulation results for lower $\left(2 f_{1}-f_{2}\right)$ third order intermodulation distortion for Fujitsu FLD3F7CZ laser, with and without using the proposed pre-distorter at bias levels $I_{b}=1.75$ and $2.5 I_{t h}, \mathrm{MD}=0.14$ and $\Delta f=10 \mathrm{MHz}[14]$.

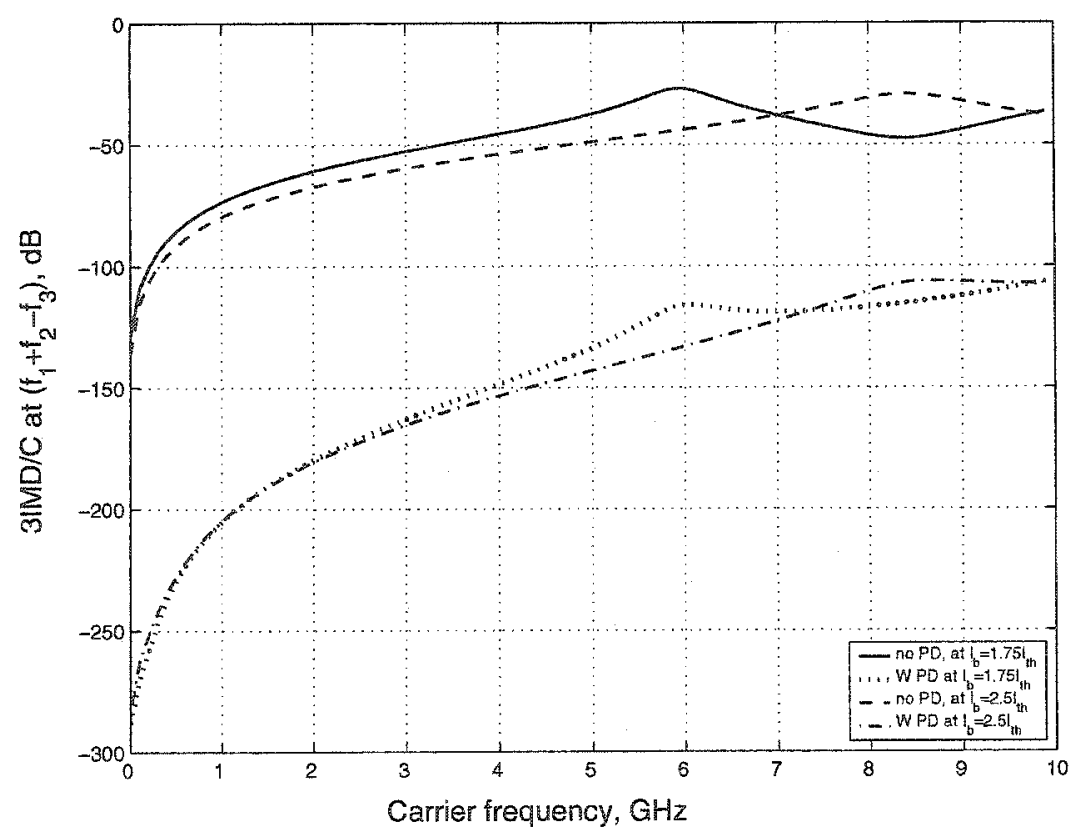

Figure 6.22: Simulation results for three tones third order intermodulation distortion relative to carrier for Fujitsu FLD3F7CZ lasers, with and without using the proposed predistorter at bias levels $I_{b}=1.75$ and $2.5 I_{t h}, \mathrm{MD}=0.14$ and $\Delta f=10 \mathrm{MHz}$. 


\section{Chapter 7}

\section{Conclusions and Recommendations for Future Research}

The semiconductor laser models based on the conventional laser rate equations were modified by incorporating a modified gain formula that includes the nonlinear functional dependence of the gain on the complex refractive index, spectral hole burning, and gain saturation. A new normalization technique was proposed, leading to an enhanced normalized laser model that enabled the implementation of a laser model using CAD tools. The modified normalized semiconductor laser model was implemented using three-port Symbolically Defined Devices (SDD) in the Agilent Advanced Design System (ADS). Accurate determination of the laser modulation response at medium to high laser bias currents was achieved using the modified laser model.

The temperature dependence of the modified gain formulation, transparent carrier density, threshold current and carrier recombination time were analytically derived. The modified normalized laser model was enhanced by including the temperature effects as described in Chapter 3. The three-port SDD model was enhanced by adding the thermal effects. Simulations of the enhanced laser model indicate that the laser threshold is an increasing function of temperature while the carrier and photon density are strong decreasing functions of temperature. Small and large signal simulations showed that modulation bandwidth and relaxation oscillation frequency are also strongly decreasing functions of temperature.

The leakage current and its effects on the laser diode performance were analytically mod- 
elled based on the diffusion theory of barrier injection. Simulation results showed that beyond a critical temperature the laser output power drops to zero. The effect of the leakage current on the carrier density is noticeable at high temperatures.

Theoretical investigation and modeling of the quantum noise in semiconductor lasers using the theory of density matrix in quantum electronics was presented. A novel self-consistent technique for relating and generating correlated photons, carrier, and phase noise sources was presented. The normalized laser model mentioned above, including the leakage current effect, was modified by adding the phase rate equation and the effects of the noise sources, leading to the normalized modified noise driven laser model. The three-port SDD laser model was enhanced by adding the noise effect and an extra port for implementing the phase rate equation. Simulation results after adding the noise operators showed the fluctuation of the photon density, carrier density, optical power and phase around their steady state values during and after the transient period for various operating conditions. Noise effects were analyzed through the study of RIN, FNS, and line width $\triangle f$. The RIN and the FNS were demonstrated and it was found that they have the same qualitative behavior for the simulation conditions and laser parameters considered. The proposed model was used to predict the laser nonlinearity and distortion levels in the frequency domain using harmonic balance simulations.

The intrinsic laser diode parameters were extracted using a proposed extraction algorithm that is based on the DC and frequency response measurement data, and the wavelength and material composition of the laser under test.

The proposed model was validated by comparing the DC, modulation response, distortion and noise of a directly modulated semiconductor laser simulation and measurement results. The comparison between the simulation with measured results confirmed that the predicted performance using the proposed model is in good agreement with the experimental results. Different laser diode linearization techniques were reviewed. A pre-distorter model was 
developed based on the inverse of the modified semiconductor laser model using Volterra series technique. Frequency and time domain Volterra pre-distorter models were derived and presented. The pre-distorter was implemented in software.

The pre-distorter was tested using a series of single, two and three tones at different bias levels over a wide range of carrier frequencies and different bias currents to assess the distortion levels of the semiconductor laser. The broadband performance of the predistorter was tested by simulating the distortion levels of two different semiconductor lasers (1.3 $\mu \mathrm{m}$ InGaAsP-InP Ortel $1510 B$ and Fujitsu FLD3F7CZ) with and without using the pre-distorter. The performance of the pre-distorter using conventional versus modified gain formulas in both the laser and pre-distorter models was discussed.

Simulation results show that the pre-distorter designed using the more accurate model proposed in Chapter 3 performs better than the conventional gain-based pre-distorter. The pre-distorter model is more sensitive to the extracted and measured laser parameters than the forward model. Simulation results show second harmonic distortion improvements for the Ortel laser averaging $40 \mathrm{~dB}$ in the frequency range up to $6 \mathrm{GHz}$, and $55 \mathrm{~dB}$ over a $10 \mathrm{GHz}$ frequency range. An average $40 \mathrm{~dB}$ reduction of the second order intermodulation distortion was achieved. It was also shown that the third order distortion is dominated by the three-tone intermodulation distortion products at $f_{1}+f_{2}-f_{3}$, and it improved by an average of $75 \mathrm{~dB}$ for the Ortel laser and $100 \mathrm{~dB}$ for the Fujitsu laser over a $10 \mathrm{GHz}$ frequency range.

For future Improvements of this work, expansion of the model is suggested by including the matching circuit and package model of the laser package and mount as well as the effect of reflected waves from the laser mirrors and the fiber pigtail. Additional coupled rate equations or cascaded models to account for Gain-Coupled (GC) or Index-Coupled (IC) lasers should also be considered.

The recommended extensions of the pre-distorter work includes implementing the pre- 
distorter using FPGA initially to be followed by high frequency and broadband circuit design of the pre-distorter and its fabrication, perhaps in the form of a single-chip. The software implementation of the pre-distorter can be extended by including an adaptive algorithm that is able to predict the variations of the output power due to variations other than those treated by the pre-distorter. 


\section{Publications}

\section{Journal Paper}

1. S. Ghoniemy, L. MacEachern, and S. Mahmoud, "Extended Robust Semiconductor Laser Modeling for Analog Optical Link Simulations," IEEE JSTQE, vol. 9, May/June 2003.

2. S. Ghoniemy, L. MacEachern, and S. Mahmoud, "Analytical Expressions, Modeling, and simulations of Intensity and Frequency Fluctuations in a Directly Modulated Semiconductor Lasers," J. of Opt. Eng., vol. 43, Jan. 2004.

3. S. Ghoniemy and L. MacEachern and S. Mahmoud, "Nonlinear Modeling and Pre-distortion Linearization for Analog Optical Transmitter," IEEE JLT. Submitted.

\section{Invited Papers}

1. S. Ghoniemy, L. MacEachern, and S. Mahmoud, "Design, Simulation and Measurements of RF/Fiber Optical Interface for Integrated Wireless/Optical Networks." IEEE CAOL'2003, Sep. 2003. Invited.

\section{Conference Papers}

1. S. Ghoniemy, L. MacEachern, and S. Mahmoud, "Large Signal Modeling for Analog Communications," Proc. SPIE, vol. TD01, pp. 139-141, May 2002.

2. S. Ghoniemy, L. MacEachern, and S. Mahmoud, "Comprehensive Modeling of Semiconductor Lasers Including the Effect of Gain Saturation." IPR'02, July 2002.

3. S. Ghoniemy, L. MacEachern, and S. Mahmoud, "Enhanced Large Signal Laser Modeling Including Thermal Effects for Analog Communications." IEEE LFNM'02, June 2002.

4. S. Ghoniemy, L. MacEachern, and S. Mahmoud, "Robust Semiconductor Laser Modeling for Analog Optical Link Simulations," IEEE NUSOD-02, 2002.

5. S. Ghoniemy, L. MacEachern, and S. Mahmoud, "Modeling and Simulations of Intensity and Frequency Fluctuations in a Directly Modulated Semiconductor Lasers." SPIE's FN'03, 1-4 June 2003.

6. S. Ghoniemy, L. MacEachern, and S. Mahmoud, "Performance Analysis and Enhancement of RF/Fiber Optical Interface for Microcellular Wireless Transceivers." WOC'03, 14-17 July 2003.

7. S. Ghoniemy, L. MacEachern, and S. Mahmoud, "Implications of Laser Nonlinearity on the Performance of Integrated Analog Wireless/Optical Networks: Modeling, Simulation and Measurements," IEEE ICECS'03, 2003.

8. S. Ghoniemy, L. MacEachern, and S. Mahmoud, "Semiconductor Laser Distortion Improvement for High Performance Analog Optical Transmission," 46 IEEE MWSCAS, 27-30 Dec., 2003. 


\section{Appendix A}

\section{Semiconductor Laser Gain}

The propagation of the optical field inside an active medium is described by the wave equation. Starting from Maxwell's equations the wave equation is given by,

$$
\nabla \times \nabla \times E=-\mu_{0} \frac{\partial}{\partial t}(\nabla \times \mathcal{H})
$$

in which $E$ and $\mathscr{H}$ are the electric and magnetic field vectors and $\mu_{0}$ is the vacuum permeability. Neglecting the free charges effect and recall Maxwell's equations in a non-magnetic dielectric medium and the vector identity relation the wave equation can be given by,

$$
\nabla^{2} E-\frac{\sigma}{\varepsilon_{o} c^{2}} \frac{\partial E}{\partial t}-\frac{1}{c^{2}} \frac{\partial^{2} E}{\partial t^{2}}=\frac{1}{\varepsilon_{o} c^{2}} \frac{\partial^{2} \mathcal{P}}{\partial t^{2}}
$$

in which $\varepsilon_{o}$ is the vacuum permittivity, $c$ is the velocity of light in free space $c=1 / \sqrt{\mu_{0} \varepsilon_{o}}$, $\sigma$ is the conductivity of the medium and $\mathcal{P}$ is the electric polarization. For an optical field with harmonic time variations, the wave equation is given by,

$$
\nabla^{2} E+k_{o}^{2}\left[1-\frac{i \sigma}{\varepsilon_{o} \omega_{o}}\right] E=-\left(\frac{k_{o}^{2}}{\varepsilon_{o}}\right) P
$$

in which $k_{o}=\omega_{0} / c=2 \pi / \lambda_{o}$ is the vacuum wave number, and $P$ is related to $E$ through the medium susceptibility $\chi(\omega)$ by, $P=\varepsilon_{o} \chi\left(\omega_{0}\right) E . \chi(\omega)$ is the susceptibility second-rank tensor that provides the refractive index and gain changes. It is given by [83],

$$
\chi\left(\omega_{0}\right)=-\int_{\mathbf{A}}^{\infty} \frac{\left(1+\mathbb{N}^{2}\right) f(\mathbb{N})}{(\mathbb{N}-i)\left(1+\mathbb{N}^{2}+\mathbb{I}\right)} d \mathbb{N}
$$

in which, $A=\left(\omega_{g}-\omega_{0}\right) \tau_{i n}, \omega_{g}$ is the band gap frequency. $\omega_{0}$ is the optical angular frequency and $\tau_{i n}$ is the intraband relaxation time (dipole relaxation time). $\mathbb{N}=\left(\omega_{t}-\omega_{0}\right) \tau_{i n}$ is the normalized detuning parameter, $\omega_{t}$ is the transition frequency, $I$ is the normalized intensity, and $f(\mathbb{N})$ is given by,

$$
f(\mathbb{N})=\Gamma d_{m}^{2} \mathrm{D}\left(\bar{a}_{11}-\bar{a}_{22}\right) /\left(\varepsilon_{o} \bar{h}\right)
$$


in which, $\mathrm{d}_{m}$ is the dipole moment, $\mathrm{D}$ is the joint density of states. $\Gamma$ appeared in this equation because $\bar{a}_{11}-\bar{a}_{22}=0$ outside the active region. For an isotropic medium $\chi(\omega)$ is scalar and can be represented by linear and nonlinear parts, $\chi\left(\omega_{0}\right)=\chi_{l}\left(\omega_{0}\right)+\chi_{n l}\left(\omega_{0}\right)$, $\chi_{l}\left(\omega_{0}\right)$ is the medium susceptibility in the absence of external pumping,

$$
\chi_{l}\left(\omega_{0}\right)=-\int_{\mathrm{A}}^{\infty} \frac{f(\mathbb{N})}{\mathbb{N}-i} d \mathbb{N}
$$

$\chi_{n l}\left(\omega_{0}\right)$ depends on the concentration of charge carriers in the active region which is function of the strength of pumping as given by [83],

$$
\chi_{n l}\left(\omega_{o}\right)=\mathbb{I} \times \int_{\mathrm{A}}^{\infty} \frac{f(\mathbb{N})}{(\mathbb{N}-i)\left(1+\mathbb{N}^{2}+\mathbb{I}\right)} d \mathbb{N}
$$

From equation (A.0.7), $\chi_{n l}\left(\omega_{0}\right)$ is a complex function of frequency and can be described through its stable poles leading to the time domain transient response [46]. $\chi_{n l}\left(\omega_{o}\right)$ can be evaluated using Cauchy's integral theorem. This can be done by taking an appropriate contour where there are no poles inside the contour, and assuming that the value of the integral comes essentially from the region where $f(\mathbb{N})<1$, while the contribution from the large contour goes to zero as its radius goes to infinity. Assuming $f(\mathbb{N})$ varies slowly in the first region the lower limit of the integration can be replaced by $(-\infty)$ and $f(\mathbb{N})$ can be approximated by,

$$
f(\mathbb{N}) \simeq f(0)+\mathbb{N} d f(\mathbb{N}) /\left.d \mathbb{N}\right|_{\mathbb{N}=0}
$$

Closing the contour in the upper half plane gives a single pole at $\mathbb{N}=-i \sqrt{1+\mathbb{I}}$ at which $\chi_{n l}(\omega)$ is given by,

$$
\chi_{n l}\left(\omega_{o}\right) \simeq \frac{i \pi f(0)}{\sqrt{1+\mathbb{I}}} \times \frac{(1-i x \sqrt{1+\mathbb{I}}) \mathbb{I}}{(1+\sqrt{1+\mathbb{I}})}
$$

in which $x=f^{\prime} / f(0)<1$, and is equal to zero if the lasing occurs at the gain peak [82], I is given by,

$$
\mathbb{I}=\left|\mathbb{E}_{o}\right|^{2} / \mathbb{I}_{s}=\mathbb{P} / \mathbb{P}_{s}=P_{\text {out }} / P_{s}
$$

in which $\left|\mathbb{E}_{o}\right|^{2}$ is the intracavity mode intensity, $\mathbb{P}$ is the intracavity photon number, $\mathbb{I}_{s}$ is the saturation intensity, $\mathbb{P}_{s}$ is the saturation photon number, $P_{\text {out }}$ is the output power, and $P_{s}$ is the saturation output power. The saturation intensity $\mathbb{I}_{s}$ is related to the saturation photon number by [117],

$$
\mathbb{P}_{s}=\varepsilon_{o} \bar{n} n_{g} V_{c} \mathbb{I}_{s} / \hbar \omega_{o} \Gamma
$$


in which $\bar{n}$ and $n_{g}$ are the effective mode refractive index and group refractive index respectively, $V_{c}$ is the volume of the active region, $\hbar=h / 2 \pi$. $\mathbb{I}_{s}$ is also a function of the intraband relaxation times $[117,182]$, as given by,

$$
\mathbb{T}_{s}=\frac{\hbar^{2}}{\mathrm{~d}_{m}^{2}\left\langle|U(\underline{r})|^{2}\right\rangle \tau_{i n}\left(\tau_{c}+\tau_{v}\right)}
$$

in which, $\tau_{c}$, and $\tau_{v}$ are the intraband relaxation times for electrons and holes respectively. Simplifying (A.0.12) by assuming $|U(r)|^{2} \approx 1$ in steady state leads to,

$$
\mathbb{I}_{s}=\frac{\hbar^{2}}{\mathrm{~d}_{m}^{2} \tau_{i n}\left(\tau_{c}+\tau_{v}\right)}
$$

\section{A.1 Index of refraction and absorption coefficient}

The time independent wave equation can be derived from (A.0.3) and given given by,

$$
\nabla^{2} \mathrm{E}+\varepsilon k_{o}^{2} \mathrm{E}=0
$$

in which $\varepsilon=\varepsilon_{r}+i \varepsilon_{i m}$ is the complex dielectric constant, $\varepsilon_{r}=\varepsilon_{b}+\operatorname{Re}\left(\chi_{n l}\right)$ and $\varepsilon_{i m}=$ $\operatorname{Im}\left(\chi_{l}+\chi_{n l}\right)+\sigma / \varepsilon_{o} \omega_{o}$ and $\varepsilon_{b}=1+\operatorname{Re}\left(\chi_{l}(\omega)\right)$ is the background dielectric constant of the un-pumped material [6]. The relation between both the index of refraction $n$ and absorption coefficient $\alpha$ and the complex dielectric constant can be found by solving the wave equation (A.1.1) for a plane wave propagating in the positive $Z$ direction, leading to,

$$
\mathbb{E}=\hat{p} \mathrm{E}_{o} U(z)=\hat{p} \mathbb{E}_{o} e^{i \bar{\alpha} z}
$$

in which $\tilde{\alpha}$ is the complex propagation constant. It can be evaluated using (A.1.2) and (A.1.1) and given as,

$$
\tilde{\alpha}=k_{o} \sqrt{\varepsilon}=k_{o} \tilde{n}
$$

in which $\tilde{n}$ is the complex index of refraction and given by,

$$
\tilde{n}=n+i\left(\alpha / 2 k_{o}\right)
$$

$\alpha$ and $n$ can be be determined by equating the complex dielectric constant with the sum of (A.1.3) and (A.1.4) as given by

$$
\varepsilon_{r}+i \varepsilon_{i m}=n^{2}+i\left(\frac{\alpha n}{k_{o}}\right)-\frac{\alpha^{2}}{4 k_{o}^{2}}
$$




$$
\varepsilon_{r}=\frac{4 k_{o}^{2} n^{2}-\alpha^{2}}{4 k_{o}^{2}} \quad \text { and } \quad \varepsilon_{i m}=\frac{\alpha n}{k_{o}}
$$

Practically $\alpha \ll n k_{0}$, this means $\alpha^{2}$ can be neglected compared to $n^{2} k_{o}^{2}$ leading to,

$$
\begin{gathered}
n=\sqrt{\varepsilon_{r}}=\sqrt{\varepsilon_{b}+\operatorname{Re}\left(\chi_{n l}\right)} \\
\alpha=\frac{k_{o} \varepsilon_{i m}}{n}=\frac{k_{o}}{n}\left\{\operatorname{Im}\left(\chi_{l}+\chi_{n l}\right)+\frac{\sigma}{\varepsilon_{o} \omega_{o}}\right\}
\end{gathered}
$$

in which $\chi_{l}\left(\omega_{0}\right)$ and $\chi_{n l}\left(\omega_{0}\right)$ are given by (A.0.6) and (A.0.9) respectively.

\section{A.2 Effect of refractive index variation on the Gain}

Both refractive index $n$ and the absorption coefficient $\alpha$ are affected by the external pumping of the semiconductor material as it is shown in equations (A.1.7) and (A.1.8). High density of charge carriers inside the active region and the shrinking of the band-gab at higher temperatures due to higher pumping levels are the main reasons for the index change $\Delta n$. Simplifying (A.1.7) leads to,

$$
n=n_{b}+\Delta n_{b c}
$$

Where $n_{b}$ is the background refractive index of the pumped material, and $\Delta n_{b}$ is the amount of change in the background refractive index in the presence of charge carriers. Equating (A.2.1) and (A.1.7) yields,

$$
\varepsilon_{b}+\operatorname{Re}\left(\chi_{n l}\right)=n_{b}^{2}+\left(2 n_{b}+\Delta n_{b c}\right) \Delta n_{b c}
$$

Assuming $\Delta n_{b}$ can be neglected compared to $n_{b}$ gives $n_{b}=\sqrt{\varepsilon_{b}}$ and $\Delta n_{b c} \approx \operatorname{Re}\left(\chi_{n l}\right) / 2 n_{b}$. The net gain $g$ can be evaluated from (A.1.8) and given by,

$$
\mathrm{g}=-\left(k_{o} / n_{b}\right) \operatorname{Im}\left(\chi_{l}+\chi_{n l}\right)
$$

Neglect the effect of the spontaneous emission, (A.2.3) gives the net gain as,

$$
\alpha=-\Gamma g+\alpha_{i n t}, \quad \alpha_{i n t}=k_{o} \sigma / n \varepsilon_{o} \omega_{o}
$$




\section{A.3 Threshold condition}

Consider a semiconductor laser of length $\mathrm{L}$ and facet reflectivities $\mathrm{R} 1$ and $\mathrm{R} 2$, the optical field propagates along the cavity length is described by (A.1.2). Its solution in steady state leads to,

$\sqrt{R_{1} R_{2}} e^{2 i \tilde{\alpha} L}=1$
Substituting from (A.1.3) and (A.1.4) into (A.3.1) yield,

$$
\sqrt{R_{1} R_{2}} e^{2 i\left\{k_{o}\left(n+i \frac{\alpha}{2 k_{o}}\right)\right\} L}=1
$$

The solution of (A.3.2) is given by,

$$
\sqrt{R_{1} R_{2}} e^{-\alpha L}=1 \quad \text { and } \quad \sin \left(2 n k_{o} L\right)=0
$$

The mirror loss $\alpha_{m}=-\alpha$ representing the amount of escaping radiation from the $F P$ cavity due to the finite facet reflectivity (absorption loss) can be derived from (A.3.3) and given as,

$$
\left.\alpha_{m}\right|_{\text {th }}=\frac{1}{2 L} \ln \left(\frac{1}{R_{1} R_{2}}\right)
$$

The threshold gain $g_{t h}$ can be derived from (A.2.4) and (A.3.4) and given as,

$$
\mathrm{g}_{t h}=\left(\alpha_{m}+\alpha_{i n t}\right) / \Gamma
$$

\section{A.4 Index nonlinearity effect on the laser gain}

Recall equations (A.2.1), (A.2.3) and (A.1.7), also assume a linear variation of both the gain and index of refraction with the carrier density $\left(\mathrm{g}(\mathbb{C})=\gamma\left(\mathbb{C}-\mathbb{C}_{t r}\right), \Delta n_{b c}=\mathbb{R} \mathbb{C}\right.$, $\mathrm{g}_{c}=\partial \mathrm{g}(\mathbb{C}) / \partial \mathbb{C}$ and $\left.\mathbb{R}=\partial n / \partial \mathbb{C}\right)[114]$ leads to,

$$
\begin{aligned}
\operatorname{Im}\left(\chi_{n l}\right) & \approx-\frac{n_{b} \gamma}{k_{o}}\left(\mathbb{C}-\mathbb{C}_{t r}\right)-\operatorname{Im}\left(\chi_{l}\right) \\
\operatorname{Re}\left(\chi_{n l}\right) & \approx 2 n_{b} \Delta n_{b c}=2 n_{b} \mathbb{R} \mathbb{C}
\end{aligned}
$$

The linear and nonlinear parts of $\chi(\omega)$ if (A.4.1a) and (A.4.1b) are used are given by,

$$
\begin{aligned}
\chi=\chi_{l}+\chi_{n l} & =\left\{\operatorname{Re}\left(\chi_{l}\right)+i \operatorname{Im}\left(\chi_{l}\right)\right\}+\left\{\operatorname{Re}\left(\chi_{n l}\right)+i \operatorname{Im}\left(\chi_{n l}\right)\right\} \\
& =\operatorname{Re}\left(\chi_{l}\right)+2 n_{b}\left\{\mathbb{R} \mathbb{C}-i n_{b} \gamma \mathbb{C} / k_{o}\right\}
\end{aligned}
$$

Further simplifications can be done by assuming that $\Delta n_{b c}$ and $g(\mathbb{C})$ can be decomposed into linear parts, $\Delta n_{b c l}, \mathrm{~g}_{L}(\mathbb{C})$, and nonlinear parts, $\Delta n_{b c n l}$ and $g_{n L}(\mathbb{C})$ leading to,

$$
\chi=2 n_{b}\left(\Delta n_{b c}-i g(\mathbb{C}) / 2 k_{o}\right)
$$




\section{A.4.1 Linear change of refractive index and gain}

Using equation (A.0.6) and decomposing it into linear and nonlinear parts, and then equating the results with the linear and nonlinear parts of equation (A.4.3) yields,

$$
\chi_{i}\left(\omega_{o}\right)=-\int_{\mathbb{A}}^{\infty} \frac{\mathbb{N} f(\mathbb{N})}{\mathbb{N}^{2}+1} d \mathbb{N}-i \int_{\mathbb{A}}^{\infty} \frac{f(\mathbb{N})}{\mathbb{N}^{2}+1} d \mathbb{N}=2 n_{b} \Delta n_{b c}-i 2 n_{b} g(\mathbb{C}) / 2 k_{o}
$$

Evaluation the integrals shown in $\left(\right.$ A.4.4) leads to $g_{L}(\mathbb{C})$ and $\Delta n_{b c l}$ as follows,

$$
\begin{aligned}
g_{L} & =\frac{k_{o}}{n_{b}} \int_{\mathrm{A}}^{\infty} \frac{f(\mathbb{N})}{\mathbb{N}^{2}+1} d \mathbb{N} \approx \frac{\pi k_{o}}{n_{b}} f(0) \approx \gamma\left(\mathbb{C}-\mathbb{C}_{t r}\right) \\
\Delta n_{b c l} & =\frac{-1}{2 n_{b}} \int_{\mathrm{A}}^{\infty} \frac{\mathbb{N} f(\mathbb{N})}{\mathbb{N}^{2}+1} d \mathbb{N} \approx-\frac{\beta_{c} g_{L}}{2 k_{o}}
\end{aligned}
$$

in which $\beta$ is line width enhancement factor and $\beta=\operatorname{Re}\left(\chi_{n l}\right) / \operatorname{Im}\left(\chi_{n l}\right) \approx-2 k_{o} \mathbb{R} / \gamma$, which is also given as $-2 k_{o} \partial n / \partial \mathbb{C} /(\partial \mathrm{g}(\mathbb{C}) / \partial \mathbb{C})$

\section{A.4.2 Nonlinear change of refractive index and gain}

The nonlinear gain and index of refraction parts can be evaluated by equating the nonlinear part of (A.4.3) with (A.0.9) and substituting from (A.4.5) and (A.4.6) as,

$$
\begin{aligned}
g_{n L} & \approx-\frac{g_{L} \mathbb{I}}{\sqrt{1+\mathbb{I}}(1+\sqrt{1+\mathbb{I}})} \\
\Delta n_{b c n l} & \approx-\frac{\Delta n_{b c l} x}{\beta} \frac{\mathbb{I}}{(1+\sqrt{1+\mathbb{I}})}
\end{aligned}
$$

From (A.4.7), the frequency dependance of $\mathrm{g}_{n L}$ is identical to that of $\mathrm{g}_{L}$. This means that the gain is reduced homogenously by the same amount over the spectral profile.

\section{A.5 Total gain function}

The total gain function is the result of the direct summation of (A.4.5) and (A.4.7) as given by,

$$
\mathrm{g}=\mathrm{g}_{L}+\mathrm{g}_{n L}=\mathrm{g}_{L}-\frac{\mathrm{g}_{L} \mathbb{I}}{\sqrt{1+\mathbb{I}}(1+\sqrt{1+\mathbb{I}})}
$$

Multiplying both sides of equation (A.5.1) by $v_{g}$ and using (A.0.10), the total gain function is given as,

$$
G(\mathbb{C}, \mathbb{P})=\frac{v_{g} g_{L}}{\sqrt{1+\mathbb{I}}}=\frac{\mathrm{v}_{g} \gamma\left(\mathbb{C}-\mathbb{C}_{t r}\right)}{\sqrt{1+\frac{\Gamma \hbar \omega_{o}}{\varepsilon_{o} \tilde{n} n_{g} V_{c} \mathbb{I}_{s}}} \mathbb{P}}
$$




\section{Appendix B}

\section{Transparent Carrier Density Temperature Dependance}

To calculate the carrier density we assume that the Bernard-Duraffourg condition is valid $\left(E_{g} \leq h f \leq \Delta E_{F}, E_{F C}\right.$ and $E_{F V}$ are the quasi-fermi energies of the conduction and valence bands and $E_{g}$ is the energy gap) and that the bands are parabolic as shown in Figure B.1. For simplicity, under charge neutrality condition, we assume that the electron ensemble has a quasi-Fermi distribution with temperature $T$ equal to the lattice temperature. At thermal equilibrium, the occupied number of states of energy $E_{\kappa}$ at absolute temperature $T$ is give as $[15]$,

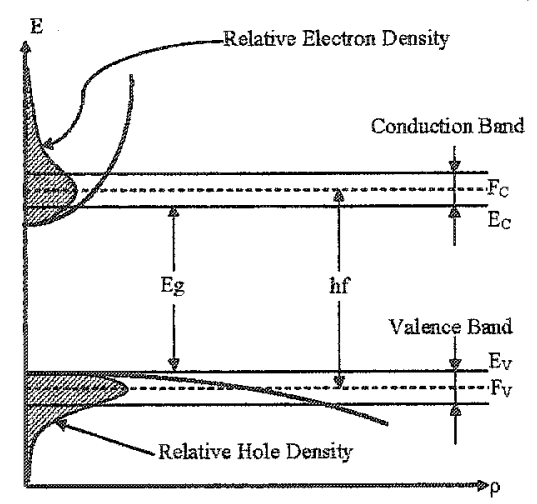

Figure B.1: Energy band diagram showing the band for net stimulated emission [15].

$$
f_{F D}(\kappa)=\left(e^{\frac{E_{\kappa}-E_{F}}{K_{B} T}}+1\right)^{-1}
$$

in which $\kappa$ is the electron state number, $K_{B}$ is the Boltzman's constant. The density of carriers can be defined as the summation of electrons over all states divided by the volume of the active region and is given by,

$$
\mathbb{C}_{t r}=\frac{2}{V_{c}} \sum_{k} f_{F D}(\kappa)=\frac{2}{V_{c}} \sum_{\kappa}\left(e^{\frac{E_{\kappa}-E_{F}}{K_{B} T}}+1\right)^{-1}
$$


The factor of 2 in (B.0.2) is to account for the carrier spin degeneracy. Replacing the summation appeared in (B.0.2) by an integration over a spherical coordinates in $\kappa$-space yield,

$$
\mathbb{C}_{i r}=\frac{2}{(2 \pi)^{3}} \int d^{3} \kappa\left(e^{\frac{E_{c K}-E_{E}}{K_{B} T}}+1\right)^{-1}
$$

where $E_{c k}$ is the energy of the state $\kappa$ within the conduction band $C$. Assuming the conduction band is spherical parabolic with effective mass $m_{c}^{*}$ and energy $E_{c k}=E_{C}+$ $\hbar \kappa^{2} / 2 m_{c}^{*}$ and the ensemble of carriers is far from degeneracy $\left(\exp \left(E_{c \kappa}-E_{F} / K_{B} T\right) \gg 1\right)$ to be able to apply Boltzman's distribution for the energy states leads to,

$$
\mathbb{C}_{t r}=\frac{2}{(2 \pi)^{3}} \int d^{3} \kappa \exp \left(\left(E_{F}-E_{C}-\frac{\hbar \kappa^{2}}{2 m_{c}^{*}}\right) / K_{B} T\right)
$$

in which $E_{C}$ is the conduction band energy, $m_{c}^{*}$ is the electron effective mass in the conduction band. Changing the integration variable in equation (B.0.4) from $\kappa$ to $v=\left(\hbar \kappa^{2} / 2 m_{c}^{*}\right)$ and simplifying the result gives,

$$
\mathbb{C}_{t r}=\frac{1}{\left(2 \pi^{2}\right)}\left(\frac{2 m_{c}^{*} K_{B} T}{\hbar^{2}}\right)^{3 / 2} e^{\frac{E_{F}-E_{C}}{K_{B} T}} \int_{0}^{\infty} \sqrt{v} e^{\left(-v / K_{B} T\right)} d v
$$

The general solution of the integration appeared in (B.0.5) is given by $[15,183]$,

$$
\int_{0}^{\infty} \sqrt{X} e^{(-a X)} d X \approx \frac{1}{2 a^{1 / 2}} \sqrt{\frac{\pi}{a}}
$$

Solving (B.0.5) using (B.0.6), $K_{B}=1.38 \times 10^{-16}[\mathrm{erg} / \mathrm{K}]$, and $\hbar=1.055 \times 10^{-27}[\mathrm{erg}]$ leads to,

$$
\mathbb{C}_{t r}=2.5 \times 10^{19}\left(m_{c}^{*} / m_{o}\right)^{3 / 2}(T / 300)^{3 / 2} e^{\frac{E_{F}-E_{C}}{K_{B} T}} \quad \mathrm{~cm}^{-3}
$$

The quasi-Fermi levels in (B.0.7) can be evaluated assuming the validity of the charge neutrality condition. This leads to,

$$
E_{F}=(3 / 4) K_{B} T \ln \left(m_{v}^{*} / m_{c}^{*}\right)+\left\{\left(E_{C}+E_{V}\right) / 2\right\}
$$

The quasi-Fermi levels can be determined by simplifying (B.0.8) and given by,

$$
\left(E_{F}-E_{C}\right)=-E_{g} / 2+(3 / 4) K_{B} T \ln \left(m_{v}^{*} / m_{c}^{*}\right)
$$


Substituting (B.0.9) into (B.0.7) leads to the transparency carrier density as a function of temperature as given by,

$$
\mathbb{C}_{t r}=2.5 \times 10^{19}\left(m_{c}^{*} / m_{o}\right)^{3 / 2}(T / 300)^{3 / 2} \exp \left\{-\frac{E_{g}}{2 K_{B} T}+\frac{3}{4} \ln \left(\frac{m_{v}^{*}}{m_{c}^{*}}\right)\right\}
$$

The temperature dependance of the energy gap is given by [184],

$$
E_{g}(T)=E_{g}(0)-4.3 \times 10^{-4} \frac{T^{2}}{T+T_{o}}
$$

in which $E_{g}$ in $\mathrm{eV}, \mathrm{T}$ is the active region temperature in $\mathrm{K}, \mathrm{T}_{o}$ is the characteristic temperature for the transparency carrier density, and $m_{0}$ is the electron rest mass. For $1.3 \mu \mathrm{m} I n_{1-x} G a_{x} A s_{y} P_{1-y}$ quaternary alloy $x=0.28$ and $y=0.6\left(E_{g} \cong 0.95 \mathrm{eV}\right)$, $m_{c}^{*} / m_{o}=0.08-0.039 y$ and $m_{v}^{*} / m_{o}=0.6-0.18 y[6,184]$.

Equation (B.0.10) can be further simplified by using (B.0.11) and expanding $T /\left(T+T_{o}\right)$ by Taylor's expansion leads to,

$$
\begin{gathered}
\mathbb{C}_{\text {tr }}(T) \approx \mathbb{C}_{\text {tro }} \exp \left(\frac{T-T_{r}}{T_{o}}\right), \\
\mathbb{C}_{\text {tro }}=2.5 \times 10^{19}\left(\frac{m_{c}^{*}}{m_{o}}\right)^{\frac{3}{2}}\left(\frac{T}{300}\right)^{\frac{3}{2}} \\
\exp \left\{\frac{3}{4} \ln \left(m_{v}^{*} / m_{c}^{*}\right)-\frac{E_{g}(0)}{2 K_{B} T}+\frac{4.3 * 10^{-4}[\mathrm{eV}]}{0.28[K] * K_{B}[\mathrm{eV} / K]}\right\}
\end{gathered}
$$




\section{Appendix C}

\section{Temperature Dependance of the Group Velocity}

The energy gap $E_{g}$ is related to the lasing wave length by (C.0.1) [6] and by (C.0.2) [184].

$$
\begin{aligned}
E_{g} & \approx 1.24 / \lambda \\
& =1.344-0.738 y+0.138 y^{2} \\
& =E_{g}(0)-4.3 \times 10^{-4} \frac{T^{2}}{T+224}
\end{aligned}
$$

in which, $E_{g}(0)=1.003[\mathrm{eV}]$, and $T$ is the device temperature in $K$.

For $1.3 \mu \mathrm{m} I n_{1-x} G a_{x} A s_{y} P_{1-y} / \operatorname{InP}$ semiconductor lasers, the mole fractions $x=0.282$ and $y=0.6$ and the index of refraction is given by, [184]

$$
\begin{aligned}
& n(x)=3.59-0.71 x+0.091 x^{2} \\
& n(y)=3.59-0.3195 y+0.01843 y^{2}
\end{aligned}
$$

Using the relation between the index of refraction and the group index given by,

$$
n_{g} \approx n-\lambda \frac{d n}{d \lambda} \approx n-\lambda \frac{d n(y)}{d y} \frac{y}{d \lambda}
$$

Using (C.0.1) and (C.0.2) and solve for $y$ yields,

$$
y_{1,2}=2.674 \pm 3.62 \sqrt{(-0.738)^{2}-4(0.138)\left(1.344-\frac{1.24}{\lambda}\right)}
$$

Substitute (C.0.6) and C.0.4b) and their derivatives in (C.0.5) leads to the group refractive index which is given by,

$$
n_{g}=n(y)-\frac{E_{g} \times(-0.3195+0.03686 y)}{\sqrt{-0.197+\frac{0.685}{\lambda}}}
$$




\section{Appendix D}

\section{Leakage Current}

Recalling the analysis introduced in Appendix B and Figure 3.10, the number of electrons at the boundary of the P-cladding layer (electrons with energy greater than the conductionband barrier height $\Delta E_{c}$ ) is given as [15],

$$
\mathbb{C}_{b}=2\left(\frac{2 \pi m_{c}^{*} K_{B} T}{h^{2}}\right)^{\frac{3}{2}} \frac{2}{\sqrt{\pi}} \int_{\epsilon_{c}}^{\infty} \frac{\sqrt{\epsilon} d \varepsilon}{1+e^{\varepsilon-\varepsilon_{f_{c}}}}
$$

Applying Bolzmann approximation for non-degenerate semiconductor lasers leads to,

$$
\mathbb{C}_{b}=N_{c c} \frac{2}{\sqrt{\pi}} \int_{\epsilon_{c}}^{\infty} \sqrt{\epsilon} e^{\left(-\epsilon+\epsilon_{f c}\right)} d \epsilon \approx N_{c c} e^{\frac{-E 1}{K_{B} T}}
$$

in which, $\epsilon_{c}=\Delta E_{c} / K_{B} T, m_{c}^{*}$ is the conduction band effective mass of the p-cladding layer and $N_{c c}$ is given by,

$$
N_{c c}=2\left(2 \pi m_{c}^{*} K_{B} T / h^{2}\right)^{3 / 2}
$$

From Figure 3.10, $E_{1}=\Delta E_{c}-E_{f c}=\Delta E_{g}-\Delta E_{v}-E_{f c}$, in which $\Delta E_{v}=E_{f v}^{\prime}-\left(-E_{f v}\right)$. Assuming non-degenerate material, the hole density can be simplified and given as,

$$
p=N_{v c} \exp \left(-E_{f v}^{\prime} /\left(K_{B} T\right)\right)
$$

Where, $N_{v c}$ is the valence band density of states for the p-cladding layer and given as,

$$
N_{v c}=2\left(2 \pi K_{B} T / h^{2}\right)^{\frac{3}{2}}\left(m_{h h}^{* 3 / 2}+m_{l h}^{* 3 / 2}\right)
$$

in which $m_{h h}^{*}$ and $m_{l h}^{*}$ are the valence band heavy and light effective masses for the pcladding layer given as, $[6,184]$

$$
\begin{aligned}
& m_{h h} / m_{0}=(1-y)\{0.97 x+0.45(1-x)\}+y\{0.45 x+0.4(1-x)\} \\
& m_{l h} / m_{\circ}=(1-y)\{0.14 x+0.12(1-x)\}+y\{0.082 x+0.026(1-x)\}
\end{aligned}
$$

From equations (D.0.2) and (D.0.3) the carrier density in the depletion region can be,

$$
\mathbb{C}_{b}=\left(N_{c c} N_{v c}\right) / p \exp \left\{-\left(\Delta E_{g}+E_{f c}+E_{f v}\right) / K_{B} T\right\}
$$


The density of holes at the boundary between the $n$-cladding layer and the active layer $\mathbb{P}_{p}$ is similar to (D.0.7). The electron and hole leakage currents densities $\left(J_{e}\right.$ and $\left.J_{p}\right)$ are related to the potential and carrier densities by the drift and diffusion relations given by [6],

$$
\begin{aligned}
& J_{e}=+q D_{e} \frac{d \mathbb{C}(x)}{d x}-q \mathbb{C}(x) \mu_{e} \psi \\
& J_{p}=-q D_{p} \frac{d \mathbb{P}(x)}{d x}+q \mathbb{P}(x) \mu_{p} \psi
\end{aligned}
$$

in which, $D_{e}, D_{p}, \mu_{e}$ and $\mu_{p}$ are the diffusion coefficients and mobilities of electrons and holes. $\mathbb{C}(x)$ and $\mathbb{P}(x)$ are the density of the electrons and holes at distance $x$ from the boundaries in the $\mathrm{p}$ and $\mathrm{n}$ cladding layers and $\psi$ is the applied electrical field. Satisfying the continuity equations by relating the divergence of $J_{e}$ and $J_{p}$ to their rate of recombination as follows,

$$
\begin{aligned}
& \nabla \cdot J_{e}-\mathbb{C}(x) / \tau_{e}=0 \\
& \nabla \cdot J_{p}+\mathbb{P}(x) / \tau_{p h}=0
\end{aligned}
$$

the solution of the $1^{\text {st }}$ order D.E. given in (D.0.8) is given as,

$$
\mathbb{C}(x)=-J_{e} /\left(q \mu_{e} \psi\right)+\mathbb{K} \exp \left(\mu_{e} x \psi / D_{e}\right)
$$

in which $K$ is the integration constant. The solution of (D.0.12) using suitable boundary conditions $\left(\left.\mathbb{C}\right|_{x=0}=\mathbb{C}_{b}\right.$ and $\left.\mathbb{C}\right|_{x=d}=0, d$ is the distance from the boundary) is given by,

$$
J_{e}=q \sqrt{\frac{\mu_{e} K_{B} T}{q \tau_{e}}}\left\{\frac{1+e^{-2 \frac{q \tau_{e}}{\mu_{e} K_{B} T}}}{1-e^{-2 \frac{q \tau_{e}}{\mu_{e} K_{B} T}}}\right\} \mathbb{C}_{b}
$$

Using $D_{e}=\mu_{e} K_{B} T / q$ and $L_{n}=\sqrt{D_{e} \tau_{e}}[6]$, equation (D.0.12) can be simplified and given by,

$$
J_{e}=q \sqrt{D_{e} / \tau_{e}}\left(\operatorname{coth} \sqrt{\tau_{e} / D_{e}}\right) \mathbb{C}_{b}
$$

The hole leakage current density can be derived in a similar way and given by,

$$
J_{p}=-q \sqrt{D_{p} / \tau_{p h}}\left(\operatorname{coth} \sqrt{\tau_{p h} / D_{p}}\right) \mathbb{P}_{b}
$$

The total Leakage current is given by,

$$
I_{\text {leak }}=\left(J_{e}+J_{p}\right) A_{a c t}
$$




\section{Appendix E}

\section{Quantum Theory of Laser and Noise Generation}

In this appendix we derive analytical expressions for the intensity and frequency/phase noise of single mode semiconductor lasers based on quantum-mechanical rate equations. Correlated photons, electrons, and phase Langevin noise sources and their auto and crosscorrelation relations are also presented along with a novel self-consistent normalized laser model that includes the correlated noise sources.

Representing a semiconductor laser as a homogenously broadened laser is perhaps the most accurate method for modeling its behavior but the complexity of such a model makes it analytically cumbersome [68]. Simplified models in which the laser is modeled using a set of energy levels are normally used. The Hamiltonian provides a suitable framework for the study of semiconductor lasers, because electrons, photons, and the interaction between them are all represented by energy operators. The effect of the heat bath contributions can also be included, which is an important consideration since electron and photon heat baths are the origin of the laser noise that causes output fluctuations [68,69]. Based on Heisenberg's equation of motion and quantum mechanics theory, each laser variable can be represented by a corresponding quantum mechanical variable. The analysis in this Appendix starts with the results of the analysis given by Sargent et al. [69] in which a system of coupled differential equations representing the time evolution of the quantum mechanical operators in a three level laser system (as given in Equations (E.0.1), (E.0.2), 
and (E.0.3)) was derived.

$$
\begin{aligned}
\frac{d l_{1}}{d t} & =I_{1}-\gamma_{1} l_{1}+\imath g \times\left(\Lambda^{c} l_{21} e^{i(\Omega-\omega) t}-h . c_{.}\right)+F_{1} \\
\frac{d l_{21}}{d t} & =-\gamma l_{21}+\imath g \times\left[\left(l_{1}-l_{2}\right)\left(\Lambda^{a} e^{i(\Omega-\omega) t}\right)\right]+F_{l} \\
\frac{d \Lambda^{a}}{d t} & =-\frac{1}{2 \tau_{p}} \Lambda^{a}-\imath g \times\left[N\left(l_{21} e^{i(\Omega-\omega) t}\right)\right]+F_{p h}
\end{aligned}
$$

In (E.0.1)-(E.0.3), $l_{1}$ and $l_{2}$ are the occupancy operators of level 1 and $2, l_{21}$ is the electron polarization operator, $I_{1}$ is the pump operator, $\Lambda^{a}$ and $\Lambda^{c}$ are the photon annihilation and creation operators, $\gamma_{1}$ and $\gamma$ are the decay rates of level 1 and the polarization phase, $\tau_{p}$ is photon life time, $g$ is the gain constant, $\omega$ is the angular transition frequency between levels 1 and $2, \Omega$ is the angular photon frequency, $F_{1}, F_{l}$ and $F_{p h}$ are the Langevin noise sources for level 1 electron polarization and photons, $l$ and $l^{*}$ are the operators of the electron dipole polarization and its conjugate, and $N$ is the total number of electrons in the laser cavity.

\section{E.1 Langevin equation and Einstein relations}

The strength of the Langevin sources (variance) depends on the laser parameters. The general form of the Langevin equation is [69],

$$
\frac{d \xi(t)}{d t}=A(t)+F(t)
$$

where $\xi(t)$ is any operator, $A(t)$ is the function represents the system, and $F(t)$ is a random Langevin function whose autocorrelation relation is given by the Markoffian approximation $\left\langle F_{\mu}(t) F_{\nu}\left(t^{\prime}\right)\right\rangle=2 A_{\mu \nu} \delta\left(t-t^{\prime}\right), A_{\mu \nu}$ is the magnitude of the fluctuations peaked about $t=t^{\prime}$ that corresponding to the maximum correlation between $\xi(t)$ and $\xi\left(t^{\prime}\right)$ while \langle\rangle indicates an ensemble average over the heat bath [68]. Taking the average of Langevin noise equation (E.1.1) and allowing that the average of the product $\xi(t) F\left(t^{\prime}\right)$ vanishes for $t<t^{\prime}$, the generalized Einstein relation is given by,

$$
2 A_{\mu \nu}=-\left\langle A_{\mu} \xi_{\nu}\right\rangle-\left\langle\xi_{\mu} A_{\nu}\right\rangle+\frac{d}{d t}\left\langle\xi_{\mu} \xi_{\nu}\right\rangle
$$


Equation (E.1.2) is used to determine the variance of the Langevin function $F(t)$ in Equation (E.1.1). Hence, (E.1.2) will be the basis for evaluating the variance of the laser noise sources.

\section{E.2 Autocorrelation of the atomic noise operators}

Based on the analysis by Sargent et al. [69], the correlation of the atomic operators $F_{1}(t), F_{l}(t)$, and $F_{p h}(t)$ appearing in $(\mathbb{E} .0 .1)-(\mathbb{E} .0 .3)$ can be written as,

$$
\begin{aligned}
\left\langle F_{1}(t) F_{1}\left(t^{\prime}\right)\right\rangle & =\frac{1}{N}\left(\left\langle I_{1}\right\rangle+\gamma_{1}\left\langle l_{1}\right\rangle\right) \delta\left(t-t^{\prime}\right) \\
\left\langle F_{l^{*}}(t) F_{l}\left(t^{\prime}\right)\right\rangle & =\frac{1}{N}\left(\left\langle I_{1}\right\rangle+\left(2 \gamma-\gamma_{1}\right)\left\langle l_{1}\right\rangle\right) \delta\left(t-t^{\prime}\right) \\
\left\langle F_{p h^{*}}(t) F_{p h}\left(t^{\prime}\right)\right\rangle & =\frac{\bar{p}_{t h}}{\tau_{p h}} \delta\left(t-t^{\prime}\right) \\
\left\langle F_{p h}(t) F_{p h^{*}}\left(t^{\prime}\right)\right\rangle & =\frac{1}{\tau_{p h}}\left(\bar{p}_{t h}+1\right) \delta\left(t-t^{\prime}\right)
\end{aligned}
$$

For semiconductor lasers at room temperature, the average thermal photon number is zero, hence $\bar{p}_{t h}$ can be set to zero in equations (E.2.3) and (E.2.4).

\section{E.3 Noise driven laser rate equations}

Coupling the atomic system to the field system by adding the electric-dipole perturbation energy to the Hamiltonian [69] using the density matrix method [70] yields the laser rate equations. As discussed in $[68,69]$, the atomic relaxation frequency $\Lambda^{a}(t)$ can be treated as a constant in the atomic equation of motion, allowing the time rate of change of $l_{1}, l_{2}$ and $l_{12}$ to be neglected. Further simplifications are made by neglecting the frequency components in the noise operators that are greater than the relaxation frequency, while still considering enough bandwidth to properly include the noise of the atomic operators with respect to the slowly varying field $\Lambda^{a}(t)$. Based on the above approximations, and using the impulse response simplification from equation (E.3.1), the solution of equation (E.0.2) is as shown 
in (E.3.2).

$$
\begin{aligned}
\delta\left(t-t^{\prime}\right) & =\frac{1}{2}[\gamma \pm \imath(\omega-\Omega)] \exp \left(\gamma \pm \imath(\omega-\Omega)\left|t-t^{\prime}\right|\right) \\
l_{21} & =\Upsilon\left\{\imath g\left(l_{1}-l_{2}\right) \Lambda^{a} \exp (\imath(\omega-\Omega) t)\right\}+\int_{-\infty}^{i} \exp (\gamma(\tau-t)) F_{l}(\tau) d \tau
\end{aligned}
$$

Substitution of equation (E.3.2) into (E.0.1) followed by multiplication by $N$ gives the electron rate equation as,

$$
\frac{d \mathbb{C}}{d t}=I_{p}-\left\{\gamma_{1}+\frac{2 g^{2}}{\gamma} \mathfrak{L}\right\} \mathbb{C}-\frac{2 g^{2}}{\gamma} \mathfrak{L}\left(\mathbb{C}-\mathbb{C}_{t r}\right) \mathbb{P}+F_{\mathbb{C}}(\mathrm{t})
$$

where $\Upsilon=1 /(\gamma+\imath(\omega-\Omega))$ is the complex line shape factor, $\mathbb{C}=N l_{1}$ is the number of electrons, $\mathbb{C}_{t r}=N l_{2}$ is the transparency carrier number, $I_{p}=N l_{1}$ is the total pump operator, and the photon number operator $\mathbb{P}=\Lambda^{c} \Lambda^{a} . \mathfrak{L}(\omega-\Omega)=\frac{1}{2} \gamma\left(\Upsilon+\Upsilon^{*}\right)=\gamma^{2} /\left(\gamma^{2}+(\omega-\Omega)^{2}\right)$ is the Lorentzian coefficient. Substituting equation $(\mathbb{E} .3 .2)$ into (E.0.3) yields,

$$
\frac{d \Lambda^{a}}{d t}=-\frac{1}{2 \tau_{p}} \Lambda^{a}+g^{2}\left(\mathbb{C}-\mathbb{C}_{t r}\right) \Upsilon \Lambda^{a}+G(t)
$$

in which $G(\mathrm{t})$ is the new noise operator. The photon rate equation given by equation (E.3.5) is derived by multiplying the photon annihilation rate equation in (E.3.4) by the photon creation operator $\Lambda^{c}$, and applying the differentiation rule of multiple operators, and then substituting from the definitions of $\mathbb{P}, \mathbb{C}, \mathbb{C}_{t r}$, and $\mathfrak{L}$ yielding,

$$
\frac{d \mathbb{P}}{d t}=-\frac{\mathbb{P}}{\tau_{p}}+\frac{2 \mathrm{~g}^{2}}{\gamma}\left(\mathbb{C}-\mathbb{C}_{t r}\right) \mathfrak{L} \mathbb{P}+\frac{2 \mathrm{~g}^{2}}{\gamma} \mathfrak{L} \mathbb{C}+F_{\mathbb{P}}(t)
$$

The electric field phase variation can be included in the density matrix using quantum mechanics, but semiclassical theory is easier and suffices for this purpose [69]. Starting with Maxwell's equations, using the analysis given by $[6,69,70,81,185]$, the wave equation may be solved using the definition of the electric field inside the laser cavity as given in (E.3.6). The electric field amplitude and phase rate equations may then be derived as 
given in equations (E.3.7) and (E.3.8).

$$
\begin{aligned}
\mathbb{E}(z, t)= & \frac{1}{2} \tilde{A} \psi(z) \exp (-2 u \pi f t) \exp (-\imath \phi(t))+c . c . \\
\frac{d \tilde{A}(t)}{d t}= & \frac{-1}{2 \tau_{p}} \tilde{A}(t)+\frac{1}{2}\left\{\frac{2 g^{2}}{\gamma}\left(\mathbb{C}-\mathbb{C}_{t r}\right) \mathfrak{L}\right\} \tilde{A}(t) \\
& +\Re \mathfrak{R}\{U(t) \exp (-\imath \phi)\} \\
\frac{d \phi}{d t}= & \frac{\beta}{2} \frac{2 g^{2}}{\gamma}(\mathbb{C}-\overline{\mathbb{C}}) \mathfrak{L}+F_{\phi}(t)
\end{aligned}
$$

$F_{\mathbb{C}}(t), F_{\mathbb{P}}(t)$ and $F_{\phi}(t)$ are the electrons, photons, and phase Langevin noise operators respectively, as given by equations (E.3.9)-(E.3.11).

$$
\begin{aligned}
F_{\mathbb{C}}(t) & =F_{1}(t)+\frac{2 g^{2}}{\gamma} \mathfrak{L C}-\imath \mathrm{g} N \exp (\imath(\omega-\Omega) t) \int_{-\infty}^{t} \exp (\gamma(\tau-t)) \Lambda^{a} F_{l}^{*}(\tau) d \tau+c . c . \\
F_{\mathbb{P}}(t) & =\Lambda^{a^{*}} F_{P h}+F_{P h}^{*} \Lambda^{a}-\frac{2 g^{2}}{\gamma} \mathfrak{L} \mathbb{C}+\imath g N \exp (\imath(\omega-\Omega) t) \int_{-\infty}^{t} \exp (\gamma(\tau-t)) \Lambda^{a} F_{l}^{*}(\tau) d \tau+c . c . \\
& =2 Q \tilde{A} \mathfrak{R e}\{U(t) \exp (-\imath \phi)\} \\
F_{\phi}(t) & =1 / \tilde{A} \mathfrak{I} \mathfrak{m}\{U(t) \exp (-\imath \phi)\}
\end{aligned}
$$

In (E.3.6)-(E.3.11), $\tilde{A}$ is the amplitude of the electric field, $\phi$ is the optical phase, and $Q=2 \varepsilon / \hbar \omega$.

\section{E.4 Independent carrier noise source generation}

One may conclude from a study of the noise sources given in equations (E.3.9)-(E.3.11) that $F_{\mathbb{I}}$ and $F_{\phi}(t)$ are not correlated. Therefore, they can be represented by two independent Gaussian noise sources with zero mean and variances given by $\left\langle F_{\mathbb{P}}(t) F_{\mathbb{P}}\left(t^{\prime}\right)\right\rangle$, and $\left\langle F_{\phi}(t) F_{\phi}\left(t^{\prime}\right)\right\rangle$ respectively. $F_{\mathbb{C}}(t)$ is correlated with both $F_{\mathbb{P}}$, and $F_{\phi}(t)$, where their crosscorrelation relations are given by $\left\langle F_{\mathbb{P}}(t) F_{\mathbb{C}}\left(t^{\prime}\right)\right\rangle$, and $\left\langle F_{\mathbb{C}}(t) F_{\phi}\left(t^{\prime}\right)\right\rangle$ respectively. Therefore, $F_{\mathbb{C}}(t)$ cannot represented by an independent Gaussian random variable.

In this Appendix, we convert the cross-correlation relations between the carrier noise source, and both the photon and phase noise sources, to alternative relations involving independent Gaussian random variables with zero mean. Application of the rule of the 
heat bath average as given in Section E.5, assuming the noise sources as operators, yields the following,

$$
\begin{aligned}
\left\langle F_{\mathbb{P}}(t) F_{\mathbb{C}}(t) F_{\mathbb{P}}\left(t^{\prime}\right) F_{\mathbb{C}}\left(t^{\prime}\right)\right\rangle= & \left\langle F_{\mathbb{P}}(t) F_{\mathbb{C}}(t)\right\rangle\left\langle F_{\mathbb{P}}\left(t^{\prime}\right) F_{\mathbb{C}}\left(t^{\prime}\right)\right\rangle+\left\langle F_{\mathbb{P}}(t) F_{\mathbb{P}}\left(t^{\prime}\right)\right\rangle\left\langle F_{\mathbb{C}}(t) F_{\mathbb{C}}\left(t^{\prime}\right)\right\rangle \\
& +\left\langle F_{\mathbb{P}}(t) F_{\mathbb{C}}\left(t^{\prime}\right)\right\rangle\left\langle F_{\mathbb{C}}(t) F_{\mathbb{P}}\left(t^{\prime}\right)\right\rangle \\
= & \left(D_{\mathbb{C}} D_{\mathbb{P} \mathbb{P}}-2 D_{\mathbb{P} \mathbb{C}}^{2}\right) \delta\left(t-t^{\prime}\right) \\
\left\langle F_{\mathbb{C}}(t) F_{\phi}(t) F_{\mathbb{C}}\left(t^{\prime}\right) F_{\phi}\left(t^{\prime}\right)\right\rangle= & \left\langle F_{\mathbb{C}}(t) F_{\phi}(t)\right\rangle\left\langle F_{\mathbb{C}}\left(t^{\prime}\right) F_{\phi}\left(t^{\prime}\right)\right\rangle+\left\langle F_{\mathbb{C}}(t) F_{\mathbb{C}}\left(t^{\prime}\right)\right\rangle\left\langle F_{\phi}(t)(t) F_{\phi}\left(t^{\prime}\right)\right\rangle \\
& +\left\langle F_{\mathbb{C}}(t) F_{\phi}\left(t^{\prime}\right)\right\rangle\left\langle F_{\phi}(t)(t) F_{\mathbb{C}}\left(t^{\prime}\right)\right\rangle \\
= & \left(D_{\mathbb{C}} D_{\phi \phi}-2 D_{\mathbb{C} \phi}^{2}\right) \delta\left(t-t^{\prime}\right)
\end{aligned}
$$

Assuming a new random variable equals the sum of equations (E.4.1) and (E.4.2) with zero mean, gives

$$
\begin{aligned}
\langle R V n\rangle & =\overline{\left\langle F_{\mathbb{P}}(t) F_{\mathbb{C}}(t) F_{\mathbb{P}}\left(t^{\prime}\right) F_{\mathbb{C}}\left(t^{\prime}\right)\right\rangle+\left\langle F_{\mathbb{C}}(t) F_{\phi}(t) F_{\mathbb{C}}\left(t^{\prime}\right) F_{\phi}\left(t^{\prime}\right)\right\rangle} \\
& =\overline{\left\langle F_{\mathbb{P}}(t) F_{\mathbb{C}}(t) F_{\mathbb{P}}\left(t^{\prime}\right) F_{\mathbb{C}}\left(t^{\prime}\right)\right\rangle}+\overline{\left\langle F_{\mathbb{C}}(t) F_{\phi}(t) F_{\mathbb{C}}\left(t^{\prime}\right) F_{\phi}\left(t^{\prime}\right)\right\rangle}=0
\end{aligned}
$$

After algebraic manipulations and substitution of equations (E.4.1) and (E.4.2) into equation (E.4.3), $\langle R V n\rangle$ is given as,

$$
\langle R V n\rangle=\left(D_{\mathbb{C C}}+\Re_{\mathbb{P C}} D_{\mathbb{P C}}+\Re_{\mathbb{C} \phi} D_{\mathbb{C} \phi}\right) \delta\left(t-t^{\prime}\right)=D_{n^{\prime} n^{\prime}} \delta\left(t-t^{\prime}\right)
$$

in which $\langle R V n\rangle$ is the autocorrelation of the new random variable $R V n, \Re_{\mathbb{P} C}=-D_{\mathbb{P C}} / D_{\mathbb{P P}}$ is the cross-correlation coefficient between $F_{\mathbb{P}}(t)$ and $F_{\mathbb{C}}(t), \Omega_{\mathbb{C} \phi}=-D_{\mathbb{C} \phi} / D_{\phi \phi}$ is the crosscorrelation coefficient between $F_{\mathbb{C}}$ and $F_{\phi(t)}$, and $D_{n^{\prime} n^{\prime}}$ is the variance of the new random variable $R V n$. RV $n$ can be simplified and given as,

$$
R V n=\left(F_{\mathbb{C}}+\mathfrak{R}_{\mathbb{P} \mathbb{C}} F_{\mathbb{P}}+\mathfrak{R}_{\mathbb{C} \phi} F_{\phi}\right)
$$

Equation (E.4.5) represents the new random variable with zero mean and variance $<R V n\rangle$. It is orthogonal to the random variables representing photons and phase noise sources. 


\section{E.5 Auto and cross-correlation relations}

The random noise functions given by equations (E.3.9)-(E.3.11) are assumed to be gaussian random variables representing the Langevin noise sources related to carriers, photons, and phase respectively with zero mean.

$$
\left\langle F_{\mathbb{C}}(t)\right\rangle=\left\langle F_{\mathbb{P}}(t)\right\rangle=\left\langle F_{\phi}(t)\right\rangle=0
$$

As discussed in Section E.2, the laser noise sources are functions of the atomic noise operators and are correlated by their variances. Consequently, the Langevin noise sources given by equations (E.3.9)-(E.3.11) are cross-correlated. These cross-correlation relations were neglected in $[6,21,34,35,186]$, but the alternative approach presented here allows for a mathematical treatment of these important terms. Mathematical manipulations of the auto-correlated and cross-correlated noise sources is cumbersome, but a suitable approximation of these terms can be chosen. Markoffian approximation [69], provide a convenient means of representing these terms, as the auto-correlation and cross-correlation functions of the noise sources may be written in terms of Dirac delta functions, as given by,

$$
\begin{aligned}
& \left\langle F_{i}(t) F_{i}\left(t^{\prime}\right)\right\rangle=D_{i i} \delta\left(t-t^{\prime}\right) \\
& \left\langle F_{i}(t) F_{j}\left(t^{\prime}\right)\right\rangle=\Re D_{i} D_{j} \delta\left(t-t^{\prime}\right)
\end{aligned}
$$

in which $D_{i}$ and $D_{j}$ are the variances of $F_{i}(t)$ and $F_{j}(t)$ respectively, and $\Re$ is the correlation coefficient. Applying this rule to our case yields,

$$
\begin{aligned}
& \left\langle F_{\mathbb{P}}(t) F_{\mathbb{P}}\left(t^{\prime}\right)\right\rangle=D_{\mathbb{P} \mathbb{P}} \delta\left(t-t^{\prime}\right) \\
& \left.\begin{array}{l}
\left\langle F_{\phi}(t) F_{\phi}\left(t^{\prime}\right)\right\rangle=D_{\phi \phi} \delta\left(t-t^{\prime}\right) \\
\left\langle F_{\mathbb{P}}(t) F_{\phi}\left(t^{\prime}\right)\right\rangle=0 \\
\left\langle F_{\mathbb{C}}(t) F_{\phi}\left(t^{\prime}\right)\right\rangle=D_{\mathbb{C} \phi} \delta\left(t-t^{\prime}\right)
\end{array}\right\}
\end{aligned}
$$

Using equation (E.3.1), the heat bath averages of operators $\langle A B C D\rangle=\langle A B\rangle\langle C D\rangle+$ $\langle A C\rangle\langle B D\rangle+\langle A D\rangle\langle B C\rangle[68]$, and using the derivations given in Section (E.4), the calcu- 
lated variances of $F_{\mathbb{P}}(t), F_{\phi}(t)$, and $F_{\mathbb{C}}(t)$ are given as,

$$
\begin{aligned}
\left\langle F_{\mathbb{C}}(t) F_{\mathbb{C}}\left(t^{\prime}\right)\right\rangle & =\left\{I_{P}+\left(\gamma_{1}+\frac{2 \mathrm{~g}^{2}}{\gamma} \mathfrak{L}\right) \mathbb{C}^{s}+\frac{2 \mathrm{~g}^{2}}{\gamma} \mathfrak{L}\left(\mathbb{C}^{s}+\mathbb{C}_{t r}\right) \mathbb{P}^{s}\right\} \delta\left(t-t^{\prime}\right) \\
& =D_{\mathbb{C C}} \delta\left(t-t^{\prime}\right) \\
\left\langle F_{\mathbb{P}}(t) F_{\mathbb{P}}\left(t^{\prime}\right)\right\rangle & =\left\{\frac{\mathbb{P}^{s}}{\tau_{p h}}+\frac{2 \mathrm{~g}^{2}}{\gamma} \mathfrak{L} N \mathbb{C}^{s}+\frac{2 \mathrm{~g}^{2}}{\gamma} \mathfrak{L}\left(\mathbb{C}^{s}+\mathbb{C}_{t r}\right) \mathbb{P}^{s}\right\} \delta\left(t-t^{\prime}\right) \\
& =D_{\mathbb{P} \mathbb{P}} \delta\left(t-t^{\prime}\right) \\
\left\langle F_{\mathbb{P}}(t) F_{\mathbb{C}}\left(t^{\prime}\right)\right\rangle & =-\left\{\frac{2 \mathrm{~g}^{2}}{\gamma} \mathfrak{L} N \mathbb{C}^{s}+\frac{2 \mathrm{~g}^{2}}{\gamma} \mathfrak{L}\left(\mathbb{C}^{s}+N_{t r}\right) \mathbb{P}^{s}\right\} \delta\left(t-t^{\prime}\right) \\
& =D_{\mathbb{P C}} \delta\left(t-t^{\prime}\right)
\end{aligned}
$$

The relation between the steady state photon number probability in terms of the transition probabilities of emission and absorption and the amplitude of the electric field $[69,81]$ is given by

$$
Q \tilde{A}^{2}= \begin{cases}\mathbb{P}^{s}+1 & \text { in case of emission (up transition) } \\ \mathbb{P}^{s} & \text { in case of absorption (down transition) }\end{cases}
$$

Combining equations (E.3.6), (E.3.8), (E.3.11), with equation (E.5.7) gives

$$
\begin{aligned}
\left\langle F_{\phi}(t) F_{\phi}\left(t^{\prime}\right)\right\rangle & =\left\langle F_{\mathbb{P}}(t) F_{\mathbb{P}}\left(t^{\prime}\right)\right\rangle / 4\left(\mathbb{P}^{s}+1\right)^{2} \\
& =\frac{D_{\mathbb{P} P}}{4\left(\mathbb{P}^{s}+1\right)^{2}} \delta\left(t-t^{\prime}\right) \approx D_{\phi \phi} \delta\left(t-t^{\prime}\right) \\
\left\langle F_{\mathbb{C}}(t) F_{\phi}\left(t^{\prime}\right)\right\rangle & =\left\langle F_{\mathbb{P}}(t) F_{\mathbb{C}}\left(t^{\prime}\right)\right\rangle / 2\left(P^{s}+1\right) \\
& =\frac{D_{\mathbb{P}}}{4\left(\mathbb{P}^{s}+1\right)^{2}} \delta\left(t-t^{\prime}\right) \approx D_{\mathbb{C} \phi} \delta\left(t-t^{\prime}\right)
\end{aligned}
$$

Where $\mathbb{C}^{s}$, and $\mathbb{P}^{s}$ are the carrier and photon steady state numbers. Relations (E.5.3), (E.5.8), and (E.5.9) are the auto-correlation and cross-correlation relations for the carrier and photon noise sources. 


\section{Appendix $\mathbb{F}$}

\section{Volterra Series}

\section{F.1 Introduction to Volterra Series}

The Spanish mathematician Vito Volterra introduced what is called Volterra series in 1930 's. Its major application was nonlinear circuit analysis by the mathematician Norbert Wiener in 1980 [187]. Volterra series are used for calculating and analyzing the distortion terms in nonlinear electronic elements. In this Appendix we investigate and analyze the distortion terms due to the laser's nonlinearity when it is modulated by an analog signal.

\section{F.2 Concepts of Volterra series}

The output of a linear, causal system with memory can be described by the fundamental convolution formulation, which is defined as the integral of the impulse response of the system and the input [188], as,

$$
y(t)=\int_{-\infty}^{\infty} h(\tau) x(t-\tau) d \tau
$$

where $x(t)$ is the input, $y(t)$ the output, and $h(t)$ the impulse response of the system. The representation of a nonlinear systems depends on whether the system has memory. A nonlinear system without memory can be represented by a Taylor series as $[148,188]$

$$
y(t)=\sum_{k=1}^{\infty} a_{k}[x(t)]^{k}
$$

where $a_{k}$ are the Taylor series coefficients.

A nonlinear system with memory can be represented by a Volterra series which combines 
the above two definitions and given as (where $\mathrm{DC}$ terms are neglected),

$$
\begin{aligned}
y(t) & =\sum_{k=1}^{\infty} \frac{1}{k !} \int_{-\infty}^{\infty} d \tau_{1} \ldots \int_{-\infty}^{\infty} d \tau_{k} g_{k}\left(\tau_{1}, \tau_{2}, \ldots, \tau_{k}\right) \prod_{r=1}^{k} x\left(t-\tau_{r}\right) \\
& =\frac{1}{1 !} \int_{-\infty}^{\infty} d \tau_{1} g_{1}\left(\tau_{1}\right) x\left(t-\tau_{1}\right) \\
& +\frac{1}{2 !} \int_{-\infty}^{\infty} d \tau_{1} \int_{-\infty}^{\infty} d \tau_{2} g_{2}\left(\tau_{1}, \tau_{2}\right) x\left(t-\tau_{1}\right) x\left(t-\tau_{2}\right) \\
& +\frac{1}{3 !} \int_{-\infty}^{\infty} d \tau_{1} \int_{-\infty}^{\infty} d \tau_{2} \int_{-\infty}^{\infty} d \tau_{3} g_{3}\left(\tau_{1}, \tau_{2}, \tau_{3}\right) x\left(t-\tau_{1}\right) x\left(t-\tau_{2}\right) x\left(t-\tau_{3}\right) \\
& +\ldots \\
& =\frac{1}{k !} \int_{-\infty}^{\infty} d \tau_{1} \int_{-\infty}^{\infty} d \tau_{2} \ldots \int_{-\infty}^{\infty} d \tau_{k} g_{k}\left(\tau_{1}, \ldots, \tau_{k}\right) x\left(t-\tau_{1}\right) \ldots x\left(t-\tau_{k}\right)
\end{aligned}
$$

$g_{n}\left(\tau_{1}, \ldots, \tau_{k}\right)$ are called the Volterra kernels of the system [187]. For $k=1, g_{1}\left(\tau_{1}\right)$ is similar to $h(\tau)$ in (F.2.1) and Equation (F.2.4) similar to (F.2.1) and known as the impulse response. Furthermore, for $k>1, g_{k}$ are known as the $k^{\text {th }}$ order (higher order) impulse response; while equations (F.2.5) - (F.2.7) are known as two-fold, three fold and $k^{\text {th }}$ fold convolution. In this work we assume that the kernels are symmetric, which means that $g_{k}\left(\tau_{1}, \ldots, \tau_{k}\right)$ have the same values regardless of the permutation of the $\tau_{1}, \ldots, \tau_{k}$.

For simplicity of calculations the nonlinear system with memory is represented by a Volterra series in the frequency domain by using Laplace or Fourier transforms. The frequency domain representation of (F.2.3), using the Fourier transforms, is given as [189],

$$
\begin{aligned}
Y(f) & =\frac{1}{1 !} G_{1}(f) X(f) \\
& +\frac{1}{2 !} \int_{-\infty}^{\infty} d f_{1} G_{2}\left(f_{1}, f-f_{1}\right) x\left(f_{1}\right) x\left(f-f_{1}\right) \\
& +\frac{1}{3 !} \int_{-\infty}^{\infty} d f_{1} \int_{-\infty}^{\infty} d f_{2} G_{3}\left(f_{1}, f_{2} f-f 1-f_{2}\right) x\left(f_{1}\right) x\left(f_{2}\right) x\left(f-f_{1}-f_{2}\right) \\
& +\cdots
\end{aligned}
$$

in which $G_{k}\left(f_{1}, \ldots, f_{k}\right)$ is given as,

$$
G_{k}\left(f_{1}, \ldots, f_{k}\right)=\int_{-\infty}^{\infty} d \tau_{1} \ldots \int_{-\infty}^{\infty} d \tau_{k} g_{k}\left(\tau_{1}, \ldots, \tau_{k}\right) e^{-j \omega_{1} \tau_{1}} \ldots e^{-j \omega_{k} \tau_{k}}
$$

Similarly, it can be given by the Laplace transform as follows,

$$
G_{k}\left(f_{1}, \ldots, f_{k}\right)=\int_{-\infty}^{\infty} d \tau_{1} \ldots \int_{-\infty}^{\infty} d \tau_{k} g_{k}\left(\tau_{1}, \ldots, \tau_{k}\right) e^{-s_{1} \tau_{1}} \ldots e^{-s_{k} \tau_{k}}
$$


where $\omega_{n}=2 \pi f_{n}, s_{n}=j \omega_{n}$ and $G_{k}$ is the $k^{\text {th }}$-order transfer function.

\section{F.3 Volterra kernels determination}

Knowing the equation that relates the input-output of certain system, the frequency and time domain Volterra kernels (coefficients of the nonlinear transfer functions) $G_{k}$ and $g_{k}$ can be determined be the techniques described below.

\section{F.3.1 Harmonic-Input (probing) method}

This method is used to determine the kernels $G_{k}$ in the frequency domain. In this method we assume that, the input is given by,

$$
x(t)=e^{\left(j \omega_{1} t\right)}+\cdots+e^{\left(j \omega_{k} t\right)}
$$

The first order Volterra transfer function $G_{1}\left(f_{1}\right)$ is the coefficient of $e^{(j \omega t)}$. The second order Volterra transfer function $G_{2}\left(f_{1}, f_{2}\right)$ is the coefficient of $e^{\left(j\left(\omega_{1}+\omega_{2}\right) t\right)}$ if we considered it as if we applied the sum of two frequency tones as input. Furthermore, similarly the $k^{t h}$ order Volterra transfer function $G_{2}\left(f_{1}, \ldots, f_{k}\right)$ is the coefficient of $e^{\left(j\left(\omega_{1}+\cdots+\omega_{k}\right) t\right)}$.

\section{F.3.2 Direct expansion method}

This method is used to determine the kernels $g_{k}$ in the time domain. In this method, the Volterra function of the system is decomposed into lower-order subfunctions of linear type $[148,188]$. Combination of these linear systems by multiplication and addition will bring the system into the general Volterra form.

Consider a second order system with kernels $g_{2}\left(\tau_{1}, \tau_{2}\right)$, according to equations (F.2.9) and (F.2.12) it can be represented by Laplace transform as follows,

$$
\begin{aligned}
G_{2}\left(s_{1}, s_{2}\right) & =\int_{-\infty}^{\infty} d \tau_{1} \int_{-\infty}^{\infty} g_{2}\left(\tau_{1}, \tau_{2}\right) \exp \left\{-\left(s_{1} \tau_{1}+s_{2} \tau_{2}\right)\right\} d \tau_{2} \\
& =G_{x}\left(s_{1}\right) G_{y}\left(s_{2}\right) G_{z}\left(s_{1}+s_{2}\right)
\end{aligned}
$$

where, $G_{x}(s), G_{y}(s)$ and $G_{z}(s)$ are the functions of the linear subsystems. Since they are linear, then, they can be represented by the convolution formula (F.2.1) with impulse 
responses $g_{x}(t), g_{y}(t)$ and $g_{z}(t)$ respectively. The block diagram representation of $G_{2}\left(s_{1}, s_{2}\right)$ is shown in Figure F.1. Similarly, the third order system with kernels $g_{3}\left(\tau_{1}, \tau_{2}, \tau_{3}\right)$ can be

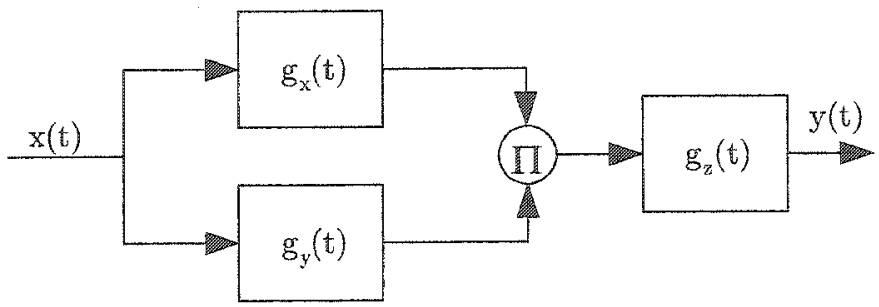

Figure F.I: Direct expansion of the second order Volterra system.

represented by Laplace transform as follows,

$$
\begin{aligned}
G_{3}\left(s_{1}, s_{2}, s_{3}\right) & =\int_{-\infty}^{\infty} d \tau_{1} \int_{-\infty}^{\infty} d \tau_{2} \int_{-\infty}^{\infty} g_{3}\left(\tau_{1}, \tau_{2}, \tau_{3}\right) \\
& \exp \left\{-\left(s_{1} \tau_{1}+s_{2} \tau_{2}+s_{3} \tau_{3}\right)\right\} d \tau_{3} \\
& =G_{x}\left(s_{1}\right) G_{y}\left(s_{2}\right) G_{k}\left(s_{3}\right) G_{z}\left(s_{1}+s_{2}\right) G_{l}\left(s_{1}+s_{2}+s_{3}\right)
\end{aligned}
$$

The block diagram representation of $G_{3}\left(s_{1}, s_{2}, s_{3}\right)$ is shown in Figure F.2.

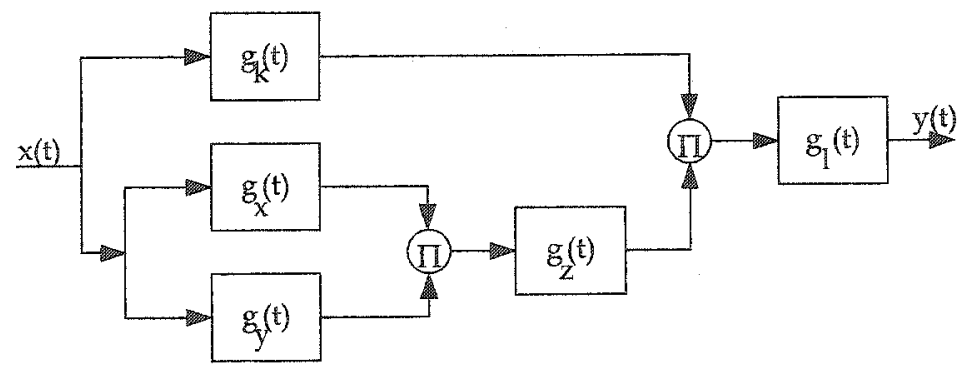

Figure F.2: Direct expansion of the third order Volterra system. 


\section{Appendix $\mathrm{G}$}

\section{Measurement Techniques}

\section{G.1 DC measurement technique}

The block diagram of the DC measurement setup shown in Figure G.1 is used for the measurements of the output optical power versus current. In this experiment, a $1.3 \mu \mathrm{m}$ InGaAsP-InP (Fujitsu FLD3F7CZ) laser was used. The laser package was mounted on an appropriate laser mount (ILX LDM4984RF) that allow direct interfacing to the bias and temperature controller. The bias and temperature control of the laser was achieved through the ILX LD controller (ILX LDC3724B) and applied via appropriate connectors in the laser mount. The laser output is connected to the Optical Measurement Head (OMH-6795) which is coupled with the Optical Multimeter (OMM-6810B) for precision power and wavelength measurements. Its wavelength accuracy is up to $\pm 0.2 \mathrm{~nm}$ in the wavelength range of 400 to $1650 \mathrm{~nm}$. For automated control, the GPIB interface allows remote programming, readout from the computer, and data gathering. A LabVIEW driver is used for this purpose.

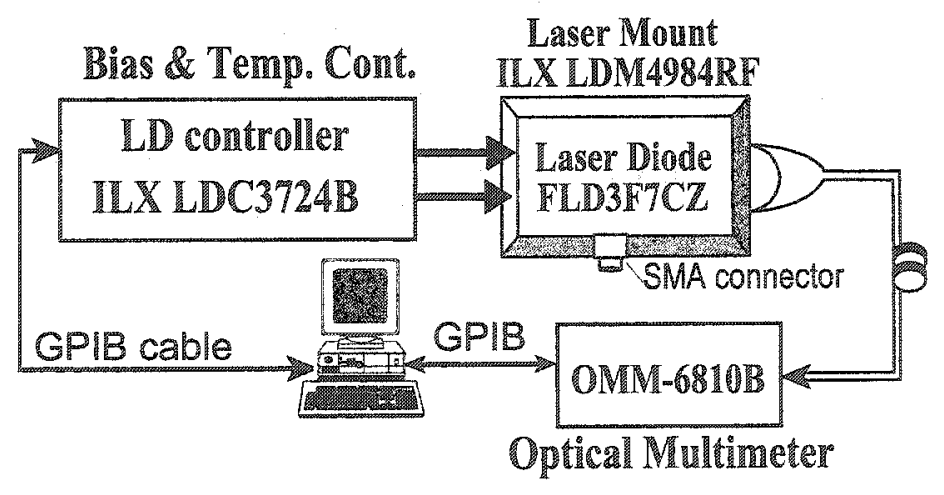

Figure G.1: Block diagram of the laser DC measurement setup. 


\section{G.2 Modulation response measurement technique}

The block diagram shown in Figure $G .2$ is used for the laser frequency response measurement. In this experiment, a $1.3 \mu \mathrm{m}$ InGaAsP-InP (Fujitsu FLD3F7CZ) butterfly packaged laser was used. The laser package was mounted on an appropriate laser mount (ILX

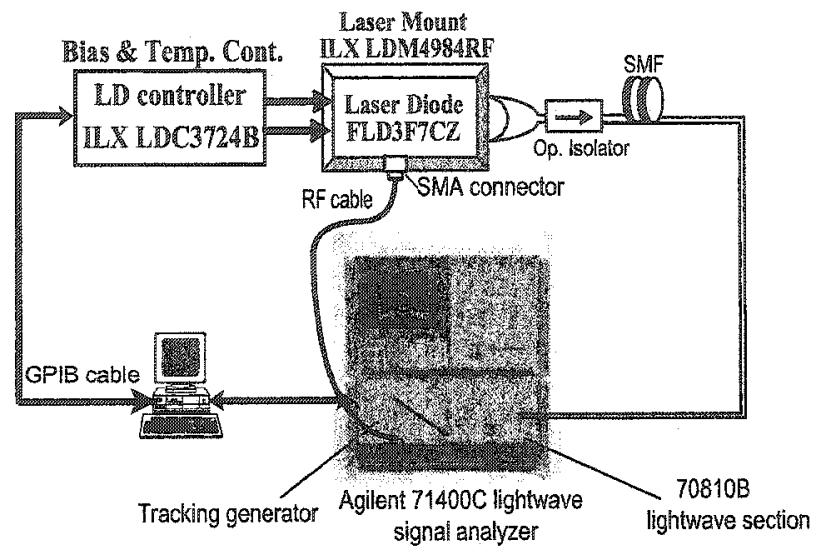

Figure G.2: Block diagram of the laser frequency response measurement setup.

LDM4984RF) that allows direct interfacing to the bias and temperature controller. The bias and temperature control of the laser was achieved through the ILX LD controller (ILX LDC3724B) and applied via appropriate connectors in the laser mount. Optical reflections were minimized by the use of an optical isolator spliced with the fiber pigtail of the laser. An Agilent RF tracking generator (70300A, $100 \mathrm{~Hz}$ to $2.9 \mathrm{GHz}$ ) and an Agilent micro-wave tracking generator (70301A, $2.7 \mathrm{GHz}$ to $18 \mathrm{GHz}$ ) from the Agilent $71400 \mathrm{C}$ lightwave signal analyzer were used to provide a sweep modulation frequency source whose frequency was synchronized with the sweep of the spectrum analyzer. The RF output of $70300 \mathrm{~A}$ was connected to the RF input of $70301 \mathrm{~A}$ to cover the full range. The output of the tracking generator was used to directly modulate the laser diode using an $R F$ cable connected to a standard RF-SMA connector in the laser mount. The mount is designed with a bias- $\mathrm{T}$ that accepts a $50 \Omega$ impedance modulation input through a standard RF-SMA connector. The modulated light enters the lightwave section in the $71400 \mathrm{C}$ system through a single-mode fiber (SMF) connected to the front-panel input connector (FC/PC). The optical power is detected, collimated and amplified, and then focused onto a $22 \mathrm{GHz}$ bandwidth InGaAs photodetector using the Agilent $70810 \mathrm{~B}$ lightwave section. The data was displayed on a 
$70004 \mathrm{~A}$ display unit and acquired through a GPIB (IEEE488.2 interface) to a personal computer as screen images and trace data using Agilent N1031A BenchLink lightwave software. In this measurement, the system turns off the system correction so that errors in both the frequency response of the electrical spectrum analyzer and the tracking generator can be calibrated.

\section{G.3 Distortion measurement technique}

The Block diagram shown in Figure G.3 is used for the laser harmonic and intermodulation distortion measurements. One, two, or multi-tone outputs from an Agilent E4433B ESG-

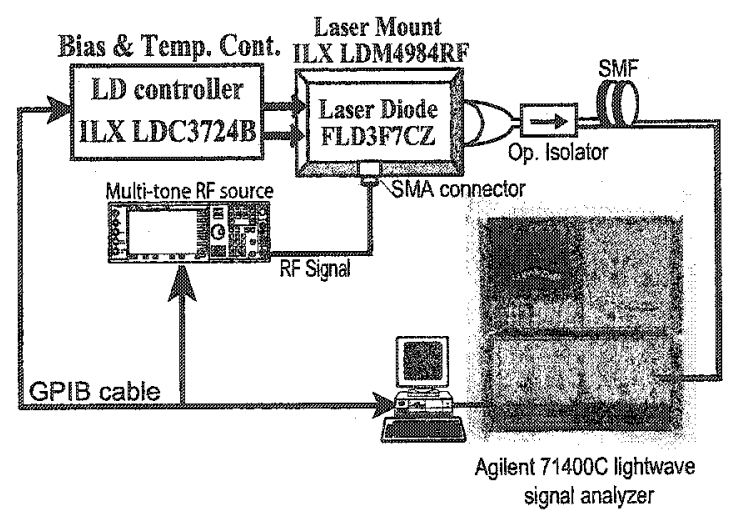

Figure G.3: Block diagram of the laser distortion measurement setup.

AP series multi-tone signal generator was used to directly modulate a $1.3 \mu \mathrm{m}$ InGaAsPInP (Fujitsu FLD3F7CZ) butterfly packaged laser. The laser package was mounted on an appropriate laser mount (ILX LDM4984RF) that allow direct interfacing to the bias and temperature controller. Its outputs are applied via appropriate connectors in the laser mount. Optical reflections were minimized by the use of an optical isolator spliced with the fiber pigtail of the laser using a suitable RF cable connected to a standard RF-SMA connector in the laser mount. The mount is designed with a bias-T that accepts a $50 \Omega$ impedance modulation input through a standard RF-SMA connector. The modulated light enters the lightwave section in the $71400 \mathrm{C}$ system through a single-mode fiber (SMF) connected to the front-panel input connector (FC/PC). The optical power is detected, 
collimated and amplified then focused onto InGaAs photodetector using the Agilent $70810 \mathrm{~B}$ lightwave section. The data was displayed on a 70004A display unit and acquired through GPIB (IEEE488.2 interface) to a personal computer as screen images and trace Data using Agilent N1031A BenchLink lightwave software.

\section{G.4 Parameters extraction algorithm}

DC and frequency response measurement data for a $1.3 \mu \mathrm{m}$ InGaAsP-InP (Fujitsu FLD3F7C2) butterfly packaged laser acquired from the optical multimeter and the spectrum analyzer through IEEE488.2 interface and used with other given parameters and mathematical relations to extract the intrinsic laser diode parameters. From the data sheet, the area of the active region was $A_{a} c t=W \times d=1 \mu m \times 0.1 \mu m=0.1 \times 10^{-12} \mathrm{~m}^{2}$, the volume was $V_{c}=24 \times 10^{-18} \mathrm{~m}^{3}$, and the mirror reluctivities were $R_{1}=0.01$ and $R_{2}=0.8$. Applying the procedure described in Appendix $C$ on the Fujitsu laser gives an average refractive index $\bar{n}=3.2$. Using equation 2.5 .7 the internal losses $\alpha_{i n t} \approx 10 \mathrm{~cm}^{-1}$. Using equation 2.5 .8 the external losses $\alpha_{m}=100 \mathrm{~cm}^{-1}$. Substitute $\alpha_{i n t}, \alpha_{m}, \bar{n}$, and the cavity length in equation 2.5 .12 the photon life time $\left(\tau_{p h} \approx 1 \mathrm{ps}\right.$. Applying the procedure explained in Appendix $B$ the transparency carrier density is, $\mathbb{C}_{\text {tro }} \approx 1 \times 10^{24} \mathrm{~m}^{-3}$.

Using the DC measurements, the threshold current $7.7 \leq I_{t h} \leq 7.9$. The relaxation oscillation frequency $f_{r}$ can be determined from the frequency response measurement results. Expressing $f_{r}$ in terms of the carrier and photon life times as in G.4.1 [6] will determine the rest of the parameters.

$$
f_{r}=\frac{1}{2 \pi} \sqrt{P_{d c} G G_{N}}
$$

$P_{d c}$ is the steady state photon density, $G$ is the gain function given by (2.5.3) and $G_{N}=$ $\frac{\partial G}{\partial N}=\Gamma v_{g} \gamma$. Solving equations (2.5.4) and (2.5.5) in steady state, and substitute from equations (2.5.6) to $(2.5 .10)$ yields,

$$
P_{d c}=\frac{\left(I-I_{t h}\right)}{q V_{c} G}
$$

Substitute from (G.4.2), (2.5.3), (refThrCurrent) and $G_{N}$ into (G.4.1) yields,

$$
f_{r}=\frac{1}{2 \pi} \sqrt{\frac{\Gamma v_{g} \gamma_{o} \mathbb{C}_{t h}}{\tau_{e}}\left(\frac{I}{I_{t h}}-1\right)}
$$


substitute equation (2.5.9) into (G.4.3) and simplify the result relates $f_{r}$ to the carriers and photon life times and is given by,

$$
f_{r}=\frac{1}{2 \pi} \sqrt{\frac{1}{\tau_{e}}\left(\frac{1}{\tau_{p h}}+\Gamma v_{g} \gamma_{o} \mathbb{C}_{i r o}\right)\left(\frac{I}{I_{t h}}-1\right)}
$$

In (G.4.4), $I, \tau_{e}, \Gamma, v_{g}$, and $\gamma_{0}$ are unknowns.

To express the measured threshold current in terms of the laser parameters, substitute equations (2.5.9) and (2.5.10) into (2.5.13) leads to,

$$
I_{t h}=\frac{q V_{c}}{\tau_{e}}\left\{\frac{1}{\tau_{p h} v_{g} \gamma_{o}}+\mathbb{C}_{t r o}\right\}
$$

Solving equations (G.4.4) and (G.4.5) for $\tau_{e}$ at specific current value $I$ and its corresponding relaxation oscillation frequency $f_{r}$. Assuming $I=20 \mathrm{~mA}$ from the frequency response measurement curve at $20 \mathrm{~mA}$, the relaxation oscillation frequency, $f_{r} \approx 6.24 G H z$. Using these values, the solution of (G.4.4) with (G.4.5) leads to the carrier life time, $\tau_{e} \approx 1$ ns. From equation (G.4.5), the term $\Gamma v_{g} \gamma_{o} \approx 9.98 \times 10^{-13} \mathrm{~m}^{3} \mathrm{~s}^{-1}$. To evaluate the gain coefficient $\mathrm{g}=v_{g} \gamma_{o}$, the confinement factor should be determined.

To determine the confinement factor, the analysis presented in Appendix $A$ is used. The transverse field distribution function can be solved by applying the appropriate continuity and boundary conditions to evaluate the TE modes inside the cavity leading to the normalized waveguide thickness as given by,

$$
D=k_{o} d \sqrt{n_{2}^{2}-n_{1}^{2}}
$$

in which $n_{2}$ and $n_{1}$ are the active and cladding layers refractive indices, $d$ is the active region thickness and $k$ is the wave number. Assuming the active medium can support only the lowest order fundamental TE mode, $D<\pi$, leads to,

$$
d \leq \frac{\lambda}{2}\left(n_{2}^{2}-n_{1}^{2}\right)^{\frac{-1}{2}} \quad\left[\mu m^{-1}\right]
$$

Using equation (A.2.4) and sections A.1 and A.2 and solve for the transverse and lateral confinement factor assuming single mode laser with cavity width $W$ small compared to the 
diffusion length as discussed in Appendix D leads to,

$$
\begin{aligned}
\alpha & =-\Gamma_{L} \Gamma_{T} g+\alpha_{i n t} \\
\Gamma_{T} & \approx D^{2} /\left(2+D^{2}\right) \\
\Gamma_{L} & \leq \approx W_{n}^{2} /\left(2+W_{n}^{2}\right) \\
W_{n} & \approx \frac{2 \pi}{\lambda} W \sqrt{n_{2}^{2}-n_{1}^{2}}
\end{aligned}
$$

in which $W_{n}$ is the normalized width of the active region.

For the $1.3 \mu \mathrm{m}$ InGaAsP-InP, $D \approx k_{o} \lambda /\left(0.95 \times 10^{-} 6\right)$ [184]. The difference between the cladding and active region index of refraction, $\Delta n_{L} \approx 0.005: 0.01$ [6]. Using the above extracted parameters for a $1.3 \mu \mathrm{m}$ InGaAsP-InP Fujitsu FLD3F7CZ laser of active region dimensions $W \times d \times L \mu m^{3}=1 \times 0.1 \times 240 \mu m^{3}, \bar{n}=3.2$ and substitute in equations (G.4.6) to (G.4.10) leads to, $\Gamma_{L} \approx 0.18$ and $\Gamma_{T} \approx 0.86$. From equation (G.4.8), the confinement factor is equal to the multiplication of the lateral and transverse confinement factors, $\Gamma=\Gamma_{L} \times \Gamma_{T} \approx 0.16$ leading to the gain coefficient $\mathrm{g}=v_{g} \gamma_{o} \approx 6.2 \times 10^{-12} \mathrm{~m}^{3} \mathrm{~s}^{-1}$.

The above extracted parameters for $1.3 \mu \mathrm{m}$ InGaAsP-InP Fujitsu FLD3F7CZ laser are summarized in table G.1.

Table G.1: Extracted parameters for a $1.3 \mu \mathrm{m}$ InGaAsP/InP Fujitsu FLD3F7CZ semiconductor laser.

\begin{tabular}{||l|l|l||}
\hline \multicolumn{1}{|c|}{ Parameter } & \multicolumn{1}{|c||}{$\begin{array}{c}\text { Symbol, } \\
\text { Unit }\end{array}$} & $\begin{array}{c}\text { Fujitsu } \\
\text { FLD3F7CZ }\end{array}$ \\
\hline \hline Operating wave length & $\lambda, \mu m$ & 1.3 \\
\hline Active region volume & $V_{c}, \mu m^{3}$ & $24 \times 10^{-18}$ \\
\hline Transparency carrier density & $\mathbb{C}_{\text {tro }}, m^{-3}$ & $1 \times 10^{24}$ \\
\hline Mode confinement factor & $\Gamma$ & 0.16 \\
\hline Gain coefficient & $\mathrm{g}=v_{g} \gamma_{o}, m^{3} s^{-1}$ & $6.2 \times 10^{-12}$ \\
\hline Spontaneous emission factor & $\beta_{s p}$ & $5 \times 10^{-5}$ \\
\hline Photon life time & $\tau_{p h}, \mathrm{~s}$ & $1 \times 10^{-12}$ \\
\hline Electrons life time & $\tau_{e}, \mathrm{~s}$ & $1 \times 10^{-12}$ \\
\hline Gain compression & $\epsilon, m^{-3}$ & $2.3 \times 10^{-24}$ \\
\hline \hline
\end{tabular}




\section{Bibliography}

[1] S. Ghoniemy, L. MacEachern, and S. Mahmoud, "Large Signal Modeing for Analog Communications," Proc. SPIE, vol. TD01, pp. 139-141, May 2002.

[2] S. Ghoniemy, L. MacEachern, and S. Mahmoud, "Enhanced Large Signal Laser Modeling Including Thermal Effects for Analog Communications." IEEE LFNM'02, June 2002.

[3] S. Ghoniemy, L. MacEachern, and S. Mahmoud, "Comprehensive Modeling of Semiconductor Lasers Including the Effect of Gain Saturation." IPR'02, July 2002.

[4] S. Ghoniemy, L. MacEachern, and S. Mahmoud, "Extended Robust Semiconductor Laser Modeling for Analog Optical Link Simulations," IEEE JSTQE, vol. 9, May/June 2003.

[5] S. M. Sze, Physics of Semiconductor Devices. New York: John Wiley and Sons, 1981.

[6] G. P. Agrawal and N. K. Dutta, Semiconductor Lasers, vol. 1. Van Nostrand Reinhold, 1993.

[7] S. Ghoniemy, L. MacEachern, and S. Mahmoud, "Robust Semiconductor Laser Modeling for Analog Optical Link Simulations," IEEE NUSOD-02, 2002.

[8] S. Ghoniemy, L. MacEachern, and S. Mahmoud, "Analytical Expressions, Modeling, and simulations of Intensity and Frequency Fluctuations in a Directly Modulated Semiconductor Lasers," J. of Opt. Eng., vol. 43, Jan. 2004.

[9] S. Ghoniemy, L. MacEachern, and S. Mahmoud, "Design, Simulation and Measurements of RF/Fiber Optical Interface for Integrated Wireless/Optical Networks." IEEE CAOL'2003, Sep. 2003. Invited.

[10] S. Ghoniemy, L. MacEachern, and S. Mahmoud, "Implications of Laser Nonlinearity on the Performance of Integrated Analog Wireless/Optical Networks: Modeling, Simulation and Measurements," IEEE ICECS'03, 2003.

[11] D. Hassin and R. Vahldieck, "Feedforward Linearization of Analog Modulated Laser Diodes: Theoretical Analysis and Experimental Verification," IEEE Trans MW Theo. and Tech., vol. 41, pp. 23762382, Dec. 1993.

[12] X. J. Meng, D. T. K. Tong, T. Chau, and M. C. Wu, "Demonstration of an Analog Fiber-Optic Link Employing a Directly Modulated Semiconductor Laser with External Light Injection," IEEE Phot. Tech. lett., vol. 10, pp. 1620-1622, Nov. 1998.

[13] D. J. M. Sabido and et al., "Improving the Dynamic Range of a Coherent AM Analog Optical Link using Cascaded Linearized Modulator," IEEE Phot. Tech. lett., vol. 7, pp. 813-815, July 1995.

[14] S. Ghoniemy, L. MacEachern, and S. Mahmoud, "Semiconductor Laser Distortion Improvement for High Performance Analog Optical Transmission," 46 IEEE MWSCAS, 2003. Submitted.

[15] M. Balkanski and R. F. Wallis, Semiconductor Physics and Applications. MiNew York: Oxford University Press, first ed., 2000. 
[16] European IT Observatory, "The e-commerce investment gap: Europe-USA eight year comparisons," tech. rep., European Telework Online, http://www.eto.org.uk/index.htm, 2003.

[17] A. Formmer and et al., "Direct Modulation and Optical Confinement Factor Modulation of Semiconductor Lasers," Appl. Phys. Lett., vol. 67, pp. 1645-1647, Sep. 1995.

[18] L. hafskjaer and A. S. Sudbo, "Modeling of Frequency Modulation Response of Semiconductor Diode Lasers," IEEE JQE, vol. 24, pp. 625-634, April 1998.

[19] W. Huang, X. Li, and T. Makino, "Analytical Formulas for Modulation Responses of Semiconductor DFB Lasers," IEEE JQE, vol. 31, pp. 842-851, May 1995.

[20] P. S. Andre and et al., "Extraction of Laser Rate Equation Parameters," SPIE proc., vol. 3572, pp. 141-146, August 1999.

[21] E. Mortazy, V. Ahmadi, and M. K. Moravevej-Farshi, "An Integrated Equivalent Circuit Model for Relative Intensity Noise and Frequency Noise Spectrum of a Multimode Semiconductor Laser," IEEE JQE, vol. 38, pp. 1366-1371, Oct. 2002.

[22] K. Czotscher and et al., "Intensity Modulation and Chirp of $1.55 \mu \mathrm{m}$ Multiple-Quantum-Well Laser Diodes: Modeling and Experimental Verification," IEEE JSTQE, vol. 5, pp. 606-612, May/June 1999.

[23] M. Chongcheawchamnan, M. J. Blewett, and I. Robertson, "Feedforward Linearization Applied to a Direct Carrier Modulation Transmitter," IEEE ISCS'2000, vol. 1, pp. 655 -658, 2000.

[24] T. Iwai, K. Sato, and K.-I. Suto, "Signal Distortion and Noise in AM-SCM Transmission System Employing the Feedforward Linearization MQW-EW External Modulator," JLT, vol. 13, pp. 16061612, Aug. 1995.

[25] B. Buxton and R. Vahldieck, "Noise and Intermodulation Distortion Reduction in an optical Feedforward Transmitter," IEEE MTT-S Digest, pp. 1105-1108, 1994.

[26] L. S. Fock and R. S. Tucker, "Reduction of Distortion in Analog Modulated Semiconductor Lasers by FeedForward Compensation," Elect. Lett., vol. 27, pp. 669-671, April 1991.

[27] P. Vankwikelberge, G. Morthier, and R. Baets, "CLADISS-A Longitudinal Multimode Model for the Analysis of the Static, Dynamic, and Stochastic Behavior of Diode Lasers with Distributed Feedback," IEEE JQE, vol. 26, no. 10, pp. $1728-1741,1990$.

[28] G. Berry, "Modeling and Design Issues in High Speed and High Power $1.3 \mu \mathrm{m}$ GaInAsP Laser Diodes," NUSOD, pp. 26-27, 2001.

[29] SILVACO International, 4701 Patrick Henry Drive, Bldg. 1, Santa Clara, CA 94054, ATLAS User's Manual, November 1998.

[30] D. E. Dodds and M. J. Sieben, "Fabry-Perot Laser Diode Modeling," IEEE Phot. Tech. Lett., vol. 7, pp. 254-256, March 1995.

[31] J. Piprek and et al., "Analog Modulation of 1.55 um Vertical-Cavity Lasers," SPIE Proc., vol. 3627, 1999.

[32] J. Piprek and et al, "Self-Consistent Analysis of High-Temperature Effects on Strained-Layer Multiquantum-Well InGaAsP-InP Lasers," IEEE JQE, vol. 36, pp. 366-374, March 2000.

[33] N. bewtra and et al., "Modeling of Quantum Well Lasers with Electro-Opto-Thermal Interactions," IEEE JSQE, vol. 1, pp. 331-340, June 1995.

[34] G. Yabre and et al., "Noise Characteristics of Single-Mode Semiconductor Laser under External Light Injection," IEEE JQE, vol. 36, pp. 385-393, March 2000. 
[35] Y. Yamamoto, "AM and FM Quantum Noise in Semiconductor Lasers- Part I: Theoretical Analysis," IEEE JQE, vol. QE-19, pp. 34-46, Jan. 1983.

[36] P. Vankwikelberge and et al., "Analysis of the Carrier-Induced FM Response of DFB Lasers: Theoretical and Experimental Case Studies," IEEE JQE, vol. 25, pp. 2237-2254, Nov. 1989.

[37] J. M. Liu, H. F. Chen, X. J. Meng, and T. B. Simpson, "Modulation Bandwidth, Noise, and Stability of a Semiconductor Laser Subject to Strong Injection Locking," IEEE Phot. Tech. Lett., vol. 9, pp. 1325-1327, Oct. 1997.

[38] S. E. Miller and D. Marcuse, "On Fluctuations and Transient in Injection Lasers," IEEE JQE, vol. QE-20, pp. 1032-1044, Sep. 1984.

[39] D. Marcuse, "Computer Simulation of Laser Photon Fluctuations: Single-Cavity Laser Results," IEEE JQE, vol. QE-20, pp. 1148-1155, Oct. 1984.

[40] P. A. Andrekson and P. Andersson, "Parasitic Element Influence on the Wide-Band Electrical Noise and Modulation Response of Semiconductor Lasers," IEEE JQE, vol. QE-23, pp. 1048-1053, June 1987.

[41] M. S. Hybertsen, "Optoelectronic Simulation: an Industrial R\&D Perspective," in NUSOD'01, 2001.

[42] C. Z. Ning and et al., "From Microscopic Physics to Advanced Laser Modeling and Simulation," in NUSOD'01, 2001.

[43] S. Ghoniemy, L. MacEachern, and S. Mahmoud, "Modeling and Simulations of Intensity and Frequency Fluctuations in a Directly Modulated Semiconductor Lasers." SPIE's FN'03, 1-4 June 2003.

[44] S. Ghoniemy, L. MacEachern, and S. Mahmoud, "Performance Analysis and Enhancement of RF/Fiber Optical Interface for Microcellular Wireless Transceivers." WOC'03, 14-17 July 2003.

[45] S. Ghoniemy and L. MacEachern and S. Mahmoud, "Measurement and Simulation of Nonlinear Distortion and Pre-distortion Linearization for Analog Optical Transmitters," IEEE JLT. Submitted.

[46] J. Carroll, J. Whiteaway, and D. Plumb, Distributed FeedBack Semiconductor Lasers, vol. PM52 of SPIE. Michael Faraday House, Six Hills Way, UK: IEE, 1998. ISBN 0852969171.

[47] Y. Suematsu and S. Arai, "Single-Mode Semiconductor Lasers for Long-Wavelength Optical Communication and Dynamics of Semiconductor Lasers," IEEE JSTQE, vol. 6, pp. 1436-1449, Nov. 2000.

[48] G. Keiser, Optical Fiber Communications. McGraw-Hill Higher Education, Thomas Casson, third ed., 2000.

[49] J. T. Verdeyen, Laser Electronics. Prentice Hall series in solid state physical electronics, Englewood Cliffs, New Jersey 07632: Prentice Hall, 3rd ed. ed., 1995.

[50] Y. Arakawa, T. Takahashi, and M. Willatzen, "Nonlinear Gain Effeets due to Carrier Heating and Spectral Hole Burning in Strained-Quantum-Well Lasers," IEEE Phot. Tech. Lett., vol. 4, pp. 682 -685 , July 1992.

[51] A. Uskov, J. Mork, and J. Mark, "Wave Mixing in Semiconductor Laser Amplifiers due to Carrier Heating and Spectral-Hole Burning," IEEE JQE, vol. 30, p. 1769, 1994.

[52] V. I. Tolstikhin and M. Willander, "Carrier Heating Effects in Dynamic-Single-Frequency GaInAsPInP Laser Diodes," IEEE JQE, vol. 31, pp. 814 -833, May 1995.

[53] M. Willatzen, A. Uskov, J. Mork, H. Olesen, B. Tromborg, and A.-P. Jauho, "Nonlinear Gain Suppression in Semiconductor Laser due to Carrier Heating," IEEE Phot. Tech. Lett., vol. 3, pp. 606609, July 1991. 
[54] W.-Z. Lin, R. W. Schoenlein, J. G. Fujimoto, and E. P. Ippen, "Femtosecond Absorption Saturation Studies of Hot Carriers in GaAs and AlGaAs," IEEE JQE, vol. 24, p. 267, 1988.

[55] J. Shah, R. F. Leheny, and R. E. Nahory, "Hot-Carrier Effects in $1.3 \mu \mathrm{m} \operatorname{In}_{1-x} \mathrm{Ga}_{x} \mathrm{As}_{y}$ P1-y $_{1-2}$ Light Emitting Diodes," Appl. Phys. Lett., vol. 39, p. 618, 1981.

[56] B. N. Gomatam and A. P. DeFonzo, "Theory of Hot Carrier Effects on Non-Linear Gain in GaAsGaAlAs Lasers and Amplifiers," IEEE JQE, vol. QE-26, pp. 1689-1704, Oct. 1990.

[57] J. Wang and H. C. Schweizer, "A Quantitative Comparison of the Classical Rate-Equation Model with the Carrier Heating Model on Dynamies of the Quantum-Well Laser: the Role of Carrier Energy Relaxation, Electron-Hole Interaction, and Auger Effect," IEEE JQE, vol. 33, pp. 1350-1359, Aug. 1997.

[58] T. V. Sarkisyan, A. T. Rosenberger, A. N. Oraevsky, and D. K. Bandy, "Gain and Carrier Temperature Response of Semiconductor Laser Media to Short Optical Pulses," JOSA, 2000.

[59] A. N. Oraevsky, T. V. Sarkisyan, and D. K. Bandy, "Nonlinear Gain and the Bistable Regime of Free-Running Oscillation in a Semiconductor Laser," Laser phys., vol. 7, p. 920, 1997.

[60] T. V. Sarkisyan, A. N. Oraevsky, A. T. Rosenberger, R. L. Rolleigh, and D. K. Bandy, "Nonlinear Gain and Carrier Temperature Dynamics in Semiconductor Laser Media," JOSA, vol. 15, p. 1107, 1998.

[61] R. Sabella, "Performance Analysis of Wireless Broadband Systems Employing Optical Fiber Links," IEEE Trans. Comm., vol. 47, pp. 715-721, May 1999.

[62] R. Sabella, M. Paciotti, and A. D. Fonzo, "Impact of Clipping and Chirping Effects on AM-VSB CATV Sub-Carrier Multiplexed Optical Systems," J. Optical Comm., vol. 19, no. 7, pp. 72-74, 1998.

[63] C. S. Li and et al, "Channel Capacity Optimization of Chirp-Limited Dense WDM/WDMA Systems Using OOK/FSK Modulation and Optical Filters," JLT, vol. 10, pp. 1148-1160, Aug. 1992.

[64] W. B. Joyce and R. W. Dixon, "Thermal Resistance of Heterostructure Lasers," J. App. Phy., vol. 46 , pp. 855-862, Feb. 1975.

[65] M. Gault and et al., "Two-Dimensional Simulation of Constricted-Mesa InGaAsP/InP BuriedHeterostructure Lasers," JQE, vol. 30, pp. 1691-1700, August 1994.

[66] C. Yi Tsai and et al., "Nonlinear Gain Coefficient in Semiconductor Lasers: Effect of Carrier Heating," IEEE JQE, vol. 32, pp. 201-212, Feb. 1996.

[67] L. Hilico, D. Touahri, F. Nez, and A. Clairon, "Narrow-Line Low-Amplitude Noise Semiconductor Laser Oscillator in the $780 \mathrm{~nm}$ Range," Rev. Sci. Instrum., vol. 65, pp. 3628-3633, Dec. 1994.

[68] D. Marcuse, "Computer Simulation of Laser Photon Fluctuations: Theory of Single-Cavity Laser," IEEE JQE, vol. QE-20, pp. 1139-1147, Oct. 1984.

[69] M. Sargent, M. O. Sgully, and W. Lamb, Laser Physics. Addison-Wesley, 1974.

[70] K. Iga and R. b. Milles, Fundamentals of Laser Optics. New York: Plenum Press, Ist ed., 1994.

[71] H. Al-Raweshidy and S. Komaki, Radio over Fiber Technologies for Mobile Communication Networks. Artech House, Incorporated, March 2002.

[72] S. Ovadia, Broadband Cable TV Access Networks: from Technologies to Applications. Upper Saddle River, NJ: Printce Hall, 2001.

[73] J. M. Senior, Optical Fiber Communications Principles and Practice. Printice Hall international series in optoelectronics, Prentice Hall, 2nd ed., 1992. 
[74] H. Haug and S. W. Koch, Quantum Theory of the Optical and Electronic Properties of Semiconductors. Singapore: Worls Scientific, 3 ed., 1994.

[75] R. E. Peierls, Quantum Theory of Solids. Oxford Classic Texts in the Physical Sciences, OXForD: OXFORD Press., February 2001.

[76] G. H. B. Thompson, Physics of Semiconductor Laser Devices. New York: Wiley, 1980.

[77] A. Yariv, Quantum Electronics. New York: John Wiley \& Sons, January 1989.

[78] S. W. K. Wng W. Chow and M. S. III, Semiconductor-Laser Physics. Berlin Heidelberg: SpringerVerlag, 1994.

[79] D. E. McCumber, "Intensity Fluctuations in the Output of CW Laser Oscillators, 1," Phy. Rev., vol. 141, pp. 306-322, Jan. 1966.

[80] H. Haug, "Quantum-Mechanical Rate Equations for Semiconductor Lasers," Phy. Rev., vol. 184, pp. 338-348, Aug. 1969.

[81] M. Ahmed, M. Yamada, and M. Saito, "Numerical Modeling of Intensity and Phase Noise in Semiconductor Lasers," IEEE JQE, vol. 37, pp. 1600-1610, Dec. 2001.

[82] G. P. Agrawal, "Effect of Gain and Index Nonlinearities on Single-Mode Dynamics in Semiconductor Lasers," IEEE JQE, vol. 26, pp. 1901-1909, Nov. 1990.

[83] G. P. Agrawal, "Specral Hole-Burning and Gain Saturation in Semiconducto Lasers: Strong-Signal Theory," J. App. Phy., vol. 63, pp. 1232-1235, Feb. 1988.

[84] G. P. Agrawal, "Effect of Non-linear Gain on Single Frequency behaviour of Semiconductor Lasers," Elect. lett., vol. 22, pp. 696-697, June 1986.

[85] H.-T. L. Y.-H. Kao, "A Predistortion Technique for Multichannel AM-VSB lightwave CATV Systems," IEEE LEOS 8th Ann. Meet. Conf. Proc., vol. 2, pp. 367 -368, 1995.

[86] P. V. M. et al., "Rate-Equation-Based Laser Model with a Single Solution Regime," JLT, vol. 15, pp. 717-730, April 1997.

[87] J. E. Bowers and et al., "High-Speed InGaAsP Constricted-Mesa Lasers," IEEE JQE, vol. QE-22, pp. 833-843, June 1986.

[88] MMET, ed., Modeling of Semiconductor Laser with Large Signal Modulation, 1996.

[89] L. M. Zhang and J. E. Carroll, "Large Signal Dynamic Model of the DFB Laser," IEEE JQE, vol. 28, pp. 604-611, March 1992.

[90] D. M. Byrne and B. A. Keating, "A Laser Diode Model Based on Temperature Dependent Rate Equation," IEEE phot. Tech. Lett., vol. 1, pp. 356-359, Nov. 1989.

[91] A. N. Oraevsky, M. M. Clark, and D. K. Bandy, "Many-Temperature Model of Laser with Dynamics," Opt. Comm., vol. 85, p. 360, 1991.

[92] K. Satzke, G. Weiser, R. Höger, and W. Thulke, "Absorption and Electroabsorption Spectra of an $\mathrm{In}_{1-x} \mathrm{Ga}_{x} \mathrm{P}_{1-y} \mathrm{As}_{y} / \mathrm{InP}$ Double Heterostructure," J. App. Phy., vol. 63, pp. 5485-5490, June 1988.

[93] Y. Zou and et al., "Experimental Study of Auger Recombination, Gain, and Temperature Sensitivity of $1.5 \mu \mathrm{m}$ Compressively Strained Semiconductor Lasers," IEEE JQE, vol. 29, pp. 1565-1575, June 1993.

[94] G. L. belenky and et al., "Role of p-Doping Profile and Regrowth on the Static Characteristics of 1.3 $\mu \mathrm{m}$ MQW InGaAsP-InP Lasers: Experimental and Modeling," IEEE JQE, vol. 35, pp. 1515-1520, Oct. 1999. 
[95] T. Keating, X. Jin, S. L. Chuang, and K. Hess, "Temperature Dependence of Electrical and Optical Modulation Responses of Quantum-Well Lasers," IEEE JQE, vol. 35, pp. 1526-1534, Oct. 1999.

[96] B.-T. Lee, R. A. Logan, R. F. Kalicek, A. M. Sergent, D. L. Coblentz, K. W. Wecht, and T. TanbunEk, "Fabrication of InGaAsP/InP Buried Hetrostructure Laser Using Reactive Ion Etching and Metalorganic Chemical Vapor Deposition," IEEE Phot. Tech. Leti., vol. 5, pp. 279-181, March 1993.

[97] H.-F. Liu, T. Kamiya, and B.-X. Du, "Temperature Dependence of Bistable InGaAsP/InP Lasers," IEEE JQE, vol. QE-22, pp. 1579-1586, Sep. 1986.

[98] L. Gillner and et al., "Semiconductor Laser Amplifier Optimization: An Analytical and Experimental Study," IEEE JQE, vol. 25, pp. 1822-1827, Aug. 1989.

[99] O. Blum and J. F. Klem, "Characteristics of GaAsSb Single-Quantum-Well Lasers Emitting Near $1.3 \mu \mathrm{m}$, IEEE Phot. Tech. Lett., vol. 12, pp. 771-773, July 2000.

[100] C.-Yi Tsai and C.-Yao Tsai, "Carrier Density Dipinning above Threshold in Semiconductor Lasers: Effect of Carrier Heating and Spectral Hole Burning," IEE Proc. Optoelectronics, vol. 144, pp. 209211, Aug. 1997.

[101] H. Lu, C. Blaauw, and T. Makino, "Single-Mode Operation Over a. Wide Temperature Range in 1.3 $\mu m$ InGaAsP-InP Distributed Feedback Lasers," JLT, vol. 14, pp. 851-859, May 1996.

[102] J. Piprek, P. Abraham, and J. E. Bowers, "Self-Consistent Analysis of High-Temperature Effects on InGaAsP/InP Lasers," IEEE Int. Symp.Compound Semiconductors, 1999.

[103] X. Li and W.-P. Huang, "Simulation of DFB Semiconductor Lasers Incorporating Thermal Effects," IEEE JQE, vol. 31, pp. 1848-1855, Oct. 1995.

[104] Z.-M. Li and T. Bradford, "A Comparative Study of Temperature Sensitivity of InGaAsP and AIGaAs MQW Lasers Using Numerical Simulations," IEEE JQE, vol. 31, pp. 1841-1847, Oct. 1995.

[105] G.-L. Tan and et al., "A two-Dimensional Nonisothermal Finite Element Simulation of Laser Diodes," JQE, vol. 29, pp. 822-835, March 1993.

[106] J. W. Scott and et al., "Modeling Temperature Effects and Spatial Hole Burning to Optimize VerticalCavity Surface Emitting Laser Performance," IEEE JQE, vol. 29, pp. 1295-1308, May 1993.

[107] I. M. P. Aarts and E. H. Sargent, "Above-Threshold Leakage in Semiconductor Lasers: An Analytical Physical Model," IEEE JQE, vol. 36, pp. 496-501, April 2000.

[108] L. L. Liou, J. L. Edel, and C. I. Huang, "Thermal Effects on the Characteristics of AlGaAs/GaAs Heterojunction Bipolar Tranisistors Using Two-Dimensional Numerical Simulation," IEEE Trans. Elec. Dev., vol. 40, pp. 35-43, Jan. 1993.

[109] Y. Yoshida and et al., "Analysis of Characteristic Temperature for InGaAsP BH Lasers with p-n-pn Blocking layers using Two-Dimensional Devices Simulator," JQE, vol. 34, no. 7, pp. 1257-1262, 1998.

[110] G. H. Song and et al., "Two-Dimensional Simulator for Semiconductor Lasers," IEDM Conf., pp. $143-146,1989$.

[111] N. Futakuchi, T. Taguchi, and Y. Nakano, "Reduction of Second- and Third-Order Harmonic Distortion by Nonlinear Absorption in Gain-Coupled Distributed Feedback Laser Diodes," IEEE JSTQE, vol. 5, pp. 463-468, May/June 1999.

[112] W. R. Smith, R. King, and B. Tuck, "Mathematical Modeling of Electrical-Optical Effects in Semiconductor Laser Operation," SIAM J. of App. Math., vol. 61, pp. 2122-2147, May 2001. 
[113] X. Jin, T. Keating, and L. Chuang, "Theory and Experiment of High-Speed Cross-Gain Modulation in Semiconductor Lasers," IEEE JOE, vol. 36, pp. 1485-1493, Dec. 2000.

[114] T. Sarkisyan, Carrier Temperature and Gain Dynamics in Semiconductor Laser Media. PhD thesis, Oklahoma State University, May 2000.

[115] Semiconductors: Group IV Elements and III-V Compounds. Berlin: Springer-Verlag, 1991. Edited by O. Madelung in Data in Science and Technology.

[116] ONLINE MATLAB Manuals .

[117] M. Asada, "Intraband Relaxation Time in Quantum-Well Lasers," IEEE JQE, vol. 25, pp. 20192026, Sep. 1989.

[118] D. M. Byrne, "Accurate Simulation of Multifrequency Semiconductor Laser Dynamics under Gigabits-per-Second Modulation," JLT, vol. 10, pp. 1086-1096, Aug. 1992.

[119] A. Mecozzi and et al, "Transient Multimode Dynamics in nearly Single-Mode Lasers," IEEE JQE, vol. 27, pp. 332-343, March 1991.

[120] J. Mork, A. Mecozzi, and G. Eisenstein, "The Modulation Response of a Semiconductor Laser Amplifier," IEEE JSTQE, vol. 5, pp. 851-860, May/June 1999.

[121] P. V. Mena, J. J. Morikuni, S.-M. Kang, A. V. Harton, and K. W. Wyatt, "A Comprehensive Circuit-Level Model of Vertical-Cavity Surface-Emitting Lasers," JLT, vol. 17, pp. 2612-2632, Dec. 1999.

[122] C.-Yi Tsai, Y. H. Lo, and R. M. Spencer, "Effects of Spectral Hole Burning, Carrier Heating, and Carrier Transport on the Small-Signal Modulation Response of Quantum Well Lasers," Appl. Phys. Lett., vol. 67, pp. 3084-3086, 1995.

[123] C. Yi Tsai, C. H. Chen, T. L. Sung, C. Yao Tsai, and J. M. Rorison, "Theoretical Modeling of Carrier and Lattice Heating Effects for Frequency Chirping in Semiconductor Lasers," Appl. Phys. Lett., vol. 74, pp. 917-919, 1999.

[124] C. Yi Tsai, C. Yao Tsai, L. F. Eastman, and Y. H. Lo, "Nonlinear Gain Coefficients in Semiconductor Lasers: Effects of Carrier Heating," IEEE JQE, vol. 32, pp. 201-212, 1996.

[125] H. Temkin and et al., "High Temperature Operation of Lattice Matched and Strained InGaAs-InP Quantum Well Lasers," IEEE Phot. Tech. lett., vol. 3, pp. 100-102, Feb. 1991.

[126] J. Stohs and et al., "Gain, Refractive Index Change, and Linewidth Enhancement Factor in BroadArea GaAs and InGaAs Quantum-Well Lasers," IEEE JQE, vol. 37, pp. 1449-1459, Nov. 2001.

[127] R. M. Spencer, J. Greenberg, L. F. Eastman, C. Yao Tsai, and S. S. OKeefe, "High-Speed Direct Modulation of Semiconductor Lasers," J. High Speed Elec. and Sys., vol. 8, pp. 41-80, 1997.

[128] H. Sun, "Calculation of Gain Saturation Caused by Photon Density Longitudinal Hole Burning in Semiconductor Lasers and in a Semiconductor Light Amplifier," Opt. Comm., vol. 127, pp. 277-282, june 1996.

[129] G. L. Tan and J. M. Xu, "Modeling of Gain, Differential Gain, Index Change, and Linewidth Enhancement Factor for Strain-Compensated QW's," IEEE phot. tech. lett., vol. 10, pp. 1386-1388, Oct. 1998.

[130] K. Liang, Q. Pan, and R. J. Green, "Nonlinear Analysis of Quantum-Well Lasers with the Effect of Carrier Transport," IEEE JQE, vol. 35, pp. 955-960, June 1999.

[131] G. Rossi, R. paoletti, and M. Meliga, "SPICE Simulation for Analysis and Design of Fast $1.55 \mu \mathrm{m}$ MQW Laser Diodes," JLT, vol. 16, pp. 1509-1516, Aug. 1998. 
[132] J.-W. Pan and J.-I. Chyi, "Theoretical Study of the Temperature Dependence of $1.3 \mu \mathrm{m}$ AlGalnAsInP Muitiple-Quantum-Well Lasers," IEEE JQE, vol. 32, pp. 2133-2138, Dec. 1996.

[133] G. R. Luevano, "Optical/Electrothermal Simulation of Integrated Optical Devices," thesis, Carleton University, Ottawa, ON, Canada, Aug. 2001.

[134] G. L. Belenky, R. F. Kazarinov, J. Lopata, S. Luryi, T. Tanbun-Elk, and P. A. Garbinski, "Direct Measurement of the Carrier Leakage Out of the Active Region in InGaAsP-InP Laser Heterostructures," IEEE Tran. Elec. Dev., vol. 42, no. 2, pp. 215-218, 1995.

[135] G. L. Belenky, D. V. Donetsky, C. L. Reynolds, R. F. Kazarinov, G. E. Shtengel, S. Luryi, and J. Lopata, "Temperature Performance of $1.3 \mu \mathrm{m}$ InGaAsP-InP Lasers with Different Profile of pdoping," IEEE Phot. Tech. Lett., vol. 9, no. 12, pp. 1558-1560, 1997.

[136] D. V. Donetsky, G. L. Belenky, D. Z. Garbuzov, H. Lee, R. U. Martinelli, G. Taylor, and S. L. J. C. Connolly, "Direct Measurements of Heterobarrier Leakage Current and Modal Gain in $2.3 \mu \mathrm{m}$ double QW p-substrate InGaAsSb/AIGaAsSb Broad Area Lasers," Elec. Lett., vol. 35, no. 4, pp. 298 $-299,1999$.

[137] G. belenky et al., "Effect of p-doping on the Temperature Dependence of Differential Gain in FP and DFB $1.3 \mu \mathrm{m}$ InGaAsP-InP Multiple-Quantum-Well Lasers," IEEE Phot. Tech. Lett., vol. 12, pp. 969-971, Aug. 2000.

[138] G. Belenky, L. Shterengas, C. L. Reynolds, M.W.Focht, M. S. Hybertsen, and B.Witzigmann, "Direct Measurement of Lateral Carrier Leakage in $1.3 \mu \mathrm{m}$ InGaAsP MQW CMBH Lasers," IEEE JQE, vol. 38, pp. 1276-1281, 2002.

[139] G. Belenky, L. Shterengas, C. W. Trussell, C. L. Reynolds, M. S. Hybertsen, and R. Menna, "Trends in Semiconductor Laser Design: Balance between Leakage, Gain and Loss in InGaAsP/InP MQW Structures," Adv. Res. Workshop, pp. 231-240, 2002.

[140] J. W. Scott, R. S. Geels, S. W. Corzine, and L. A. Coldren, "Modeling Temperature Effects and Spatial Hole Burning to Optimize Vertical-Cavity Surface-Emitting Laser Performance," IEEE JQE, vol. 29, no. 5, pp. $1295-1308,1993$.

[141] G. P. Agrawal and C. M. Bowden, "Concept of Linewidth Enhancement Factor in Semiconductor Lasers: Its Usefulness and Limitations," IEED Photonic Technology Letters, vol. 5, pp. 640-642, June 1993.

[142] J. B. Gao, S. K. Hwang, and J. M. Liu, "Effects of Intrinsic Spontaneous Noise on the Nonlinear Dynamics of an Optically Injected Semiconductor Lasers," Phy. Rev., vol. 59, pp. 1582-1585, Feb. 1999.

[143] A. I. Khinchin, Mathematical Foundations of Statistical Mechanics. New York: Dover, 1949.

[144] A. I. Khinchin, Analytical Foundations of Physical Statistics. Delhi: Hindustan, 1961.

[145] P. Billingsley, Ergodic Theory and Information. New York: Wiley, 1965.

[146] D. Ornstein, Ergodic Theory, Randomness, and Dynamical Systems. New Haven: Tal University Press, 1974.

[147] D. Derickson, Fiber Optics Test and Measurement. 1998.

[148] S. A. Maas, Nonlinear Microwave Circuits. IEEE press, 1998.

[149] X. N. Fernando and A. B. Sesay, "Higher Order Adaptive Filter Based Predistortion for Nonlinear Distortion Compensation of Radio over Fiber Links," IEEE Transactions on Electron Devices, vol. 1, pp. 367-371, 2000. 
[150] M. Sieben, "Semiconductor Laser Diode Modeling and Linearization by Predistortion," Master's thesis, U. of Saskatchewan, Aug. 1994.

[151] R. Sabella and M. Avattaneo, "Impact of Clipping and Chirping Pffects on Hybrid CATV SubCarrier Multiplexed Optical Systems," vol. 1, Nov. 1996. p 354-355.

[152] M.-B. Bibey, F. Deborgies, M. Krakowski, and D. Mongardien, "Very Low Phase-Noise Optical Link-Experiments and Theory," IEEE Tran. MW Theo. Tech., vol. 47, pp. 2257-2262, Dec. 1999.

[153] M. Faulkner and M. johansson, "Adaptive Linearization using Predistortion-Experimental Results," IEEE trans. on Weh. Tech., vol. 43, pp. 323 332, May 1994.

[154] H.-H. Lu, Y.-P. Lin, and M.-C. Lin, "Nonlinear Distortion Analysis of Directly Modulated DFB Laser Diode in CATV Systems," J Opt. Comm., vol. 22, no. online 26, p. 707(3), 2001.

[155] T. E. Darcie and G. E. Bodeep, "Lightwave Subcarrier CATV Transmission Systems," IEEE Trans MW Theo Tech., vol. 38, pp. 524-533, May 1990.

[156] T. E. Darcie and et al., "Fiber Optic Device Technology for Broadband Analog Video Systems," IEEE LCS, vol. 1, pp. 46-52, Feb. 1990.

[157] X. N. Fernando and A. B. Sesay, "Look-Up Table Based Adaptive Predistortion for Dynamic Range Enhancement in a Radio over Fiber Links," IEEE Conf. on comm., comp. and SP, pp. 26-29, 1999.

[158] R. S. Tucker, "Linearization Techniques For Wideband Analog Transmitters," LEOS'92 Sum. Meet. Digest, pp. $54-55,1992$.

[159] D. Hassin and R. Vahldieck, "Improved Feedforward Linearization of Laser Diodes - Simulation and Experimental Results," IEEE MTT-S Digest, pp. 727-730, 1993.

[160] Agere systems, "RF and Microwave Fiber-Optic Design Guide," application note, Agere systems Inc., April 2001.

[161] L. S. Fock and R. S. Tucker, "Simultaneous Reduction of Intensity Noise and Distortion in Semiconductor Lasers by FeedForward Compensation," Elect. Lett., vol. 27, pp. 1297-1299, July 1991.

[162] K. Tsukamoto, S. Fujii, P. Sanjo, and S. Komaki, "Fiber-optic Microcellular Communication System using Frequency Modulated LD and Optical Discriminator and its Nonlinear Distortion Suppression," IEEE PIMRC'97, no. 3, pp. 984 -988, 1997.

[163] J. Tsimbinos and K. V. Lever, "Nonlinear Compensation using Orthogonal Inverses: Broadband Inputs and Robustness Issues," TENCON '96, vol. 2, pp. 816-821, 1996.

[164] J. Tsimbinos and K. V. Lever, "The Computational Complexity of Nonlinear Compensators Based on the Volterra Inverse," 8th IEEE Sig. Process. Conf., pp. $387-390,1996$.

[165] Y. Ueno and M. Kajitani, "Color TV Transmission using Light Emitting Diode," NEC, pp. 15-20, Oct. 1974.

[166] H. Gysel and M. Ramachandran, "Electrical Predistortion to Compensate for Combined Effect of Laser Chirp and Fiber Dispersion," Elect. Lett., vol, 27, pp. 421-423, Feb. 1991.

[167] B. W. et al., "Analogue Optical Fiber Communications," IEE, 1995. London.

[168] L. Roselli and et al., "Predistortion Circuit Design for II and III Order Simultaneous Linearization in Multiservice Telecommunications Apparatuses," IEEE MTT-S Digest, pp. 1711-1714, 2002.

[169] Y. Chiu and et al., "Broadband Electronic Linearizer for Externally Modulated Analog Fiber-Optic Links," IEEE Phot. Tech. Lett., vol. 11, pp. 48-50, Jan. 1999.

[170] X. J. Meng, A. Yacoubian, and J. H. Bechtel, "Electro-optical Predistortion Technique for Linearization of Mach-Zehnder Modulators," Elec. Lett., vol. 37, pp. 1545-1547, Dec. 2001. 
[17i] G. C. Wilson, "Optimized Predistortion of Over Modulated Mach-Zehnder Modulators with Multicarrier Input," IEEE Phot. Tech. lett., vol. 9, pp. 1535-1537, Nov. 1997.

[172] G. Steiner, S. Hunziker, and W. Baechtold, "Reduction of $3^{\text {rd }}$ Order Intermodulation of a Semiconductor Laser by an Adaptive Low-Cost Predistortion Circuit at $1.8 \mathrm{GHz}$," IEEE LEOS Meet. RF Phot. for CATV and HFC Sys., July 1999.

[173] X. J. Meng, T. Chau, D. T. K. Tong, and M. C. Wu, "Suppression of Second harmonic Distortion in Directly Modulated Distributed Feedback Lasers by External Light Injection," Elec. Lett., vol. 34, pp. 2040-2041, Oct. 1998.

[174] H.-D. Jung and S.-K. Han, "Nonlinear Distortion Suppression in Dual Parallel Analog Modulation of Distributed Feedback-LD," IEEE MWP'00, pp. 190-193, 2000.

[175] H.-D. Jung, D.-H. Jeon, and S.-K. Han, "Linearity Enhancement of an Electro Absorption Modulated Laser by Dual-Parallel Modulation," IEEE Pho. Tech. lett., vol. 14, pp. 462-464, April 2002.

[176] A. V. de Grijp and et al, "Novel Electro-Optical Feedback Technique for Noise and Distortion Reduction in High-Quality Analog Optical Transmission Video Signals," Elec. Lett., vol. 17, pp. 361362, May 1981.

[177] M. Sekita and et al, "TV Video Transmission by Analog Baseband Modulation of $1.3 \mu \mathrm{m}$ Band Laser Diode," 6 ${ }^{\text {th }}$ Europ. Conf. Opt. Comm., pp. 394-396, 1980.

[178] M. Gans, J. E. Johnson, P. A. Morton, J. W. Sulhoff, T. Tanbun-Ek, G. C. Wilson, and T. H. W. J. L. Zyskind, "Predistortion of Electroabsorption Modulators for Analog CATV Systems at $1.55 \mu$ m," JLT, vol. 15, pp. 1654 -1662, Sep. 1997.

[179] G. C. Wilson, "Optimized Predistortion of Overmodulated Mach-Zehnder modulators with Multicarrier Input," IEEE Phot. Tech. Lett., vol. 9, pp. 1535 -1537, Nov. 1997.

[180] E. Bedrosian and S. O. Rice, "The Output Properties of Volterra System (Nonlinear Systems with Memory) Driven by Harmonic and Gaussian Inputs," Proc. of IEEE, vol. 59, pp. 1688-1707, Dec. 1971.

[181] H. M. Salgado, J. M. Ferreira, and J. J. O'Reilly, "Extraction of Semiconductor Intrinsic Laser Parameters by Intermodulation Distortion Analysis," IEEE phot. Tech. Lett., vol. 9, pp. 133-1133, Oct. 1997.

[182] G. P. Gray, A. Ryan, and G. P. Agrawal, "Noise in Semiconductor Lasers and its Reduction by High-frequency Injection," LEOS '92, pp. 333 -333, 1992.

[183] L. D. Landau, J. B. Sykes (Translator), M. J. Kearsley (Translator), and E. M. Lifshitz, Statistical Physics (Course of Theoretical Physics), vol. 1. OXFORD: Butterworth Heinemann, 3 ed., 1999.

[184] M. Levinshtein, S. Rumyantsev, and M. Shur, Hand Book on Semiconductor Parameters, vol. II. Farrer Road, PO Box 128, Singapor 912805: World Scientific, first ed., 1999.

[185] A. Murakami, J. Ohtsubo, and Y. Liu, "Stability Analysis of Semiconductor Laser with PhaseConjugate Feedback," IEEE JQE, vol. 33, pp. 1825-1831, Oct. 1997.

[186] S. M. K. Thiyagarajan and A. F. J. Levi, "Noise in Voltage-Biased Scaled Semiconductor Laser Diodes," Solid State Elec., vol. 43, pp. 33-39, 1999.

[187] N. Wiener, Nonlinear Problems in Random Theory. Mass. M.I.T. Press., 1958.

[188] M. Schetzen, The Volterra and Wiener Theories of Nonlinear Systems. New York: John Wiley \& Sons, 1980.

[189] D. D. Weiner and J. F. Spina, Sinusoidal Analysis and Modeling of Weakly Nonlinear Circuits. New York: Van Nostrand Reinhold, 1980. 